\title{
Two-Dimensional Electronic Spectroscopy of the Photosystem II D1D2-cyt.b559 Reaction Center Complex
}

\author{
by \\ Jeffrey Allen Myers \\ A dissertation submitted in partial fulfillment \\ of the requirements for the degree of \\ Doctor of Philosophy \\ (Physics) \\ in The University of Michigan \\ 2010
}

Doctoral Committee:

Assistant Professor Jennifer P. Ogilvie, Chair

Professor Paul R. Berman

Professor Timothy A. McKay

Professor Roseanne J. Sension

Assistant Professor Kevin J. Kubarych 
(C) Jeffrey Allen Myers 2010

All Rights Reserved 
To my parents, Dan and Tina Myers 


\section{ACKNOWLEDGEMENTS}

My time at the University of Michigan has been spent learning a great deal about myself and the people around me, as well as the intricacies of physics. As an undergraduate, I never guessed what it really means to get a $\mathrm{Ph}$.D. It has indeed been an adventure of great scope, a keen expansion of my knowledge and problem-solving capabilities, and a refinement of my identity as a scientist. But to stop there without acknowledging the many, many people who have helped me over the years would be a great injustice.

First, I would like to thank everyone in Professor Ogilvie's group, all of whom brought some sunshine into a lab which, despite being on the corner of the fourth floor, has no windows. Dr. Sarah Nichols has my eternal gratitude for lending me her ${ }^{A} T_{E} X b o o k$ and example code to get me started up the typesetting learning curve, without which I would have been hopelessly lost in formatting. Kristin Lewis has been by my side in the lab from day one as my lab partner and academic counterpart. Without her help, humor, and ability, I wouldn't have made it through my own blunders, and the overnight data sessions would have been far lonelier. Dr. Patrick Tekavec has always been the one to go to for questions of the sticky theoretical issues when I have trouble wrapping my mind around the complex interplay of pulses, and he seems to somehow be an expert at tweaking those parts of the laser system that I have the most trouble with. Franklin Fuller has challenged me over the last couple of years with difficult questions that a senior grad student should nevertheless be able to answer for a younger grad student, and his mastery of Tolkien's elvish 
script is unmatched in the lab. Then there are Brandon and Meredith, with whom I never really had the opportunity to work closely, despite being on opposite sides of a curtain for much of the time. We have gotten to know each other, though, for which I'm thankful. Finally, I give my best wishes to Jimmy and Dan, the next generations of $2 \mathrm{D}$ spectroscopy students, who will no doubt reach far beyond the present work.

As for Professor Ogilvie herself, I could not ask for a better advisor. Jennifer has the kind of sincere intellectual curiosity that stimulates her students to want to be better scientists. I look up to her for her tenacity in research, her ability to find novel solutions when others would be frustrated, and the sheer depth of her knowledge. How she keeps up with so many different studies and research topics across a field that changes so rapidly, I may never understand, but I am always thankful that she never has to think very hard to tell me just what papers I should be reading when I have a question.

There are a number of people, mainly along the fourth floor hallway of Randall, who have kept me sane over the years and generally been good friends: Jessica, who taught me about the NOPA and has shared more than a few coffee runs with me; David, who is always good for comic relief and serious discussion, often at the same time, and who has excellent taste in tv shows; and Gerhard, the friendliest postdoc I've ever met. I also should thank my friends and roommates, Josh and Bernadette, the only people I've ever liked well enough to live with for three years.

I owe a great debt of gratitude to Professor Yocum for personally teaching us how to extract our photosystem II samples and for making himself and his lab ever available to myself and the other students. Without his help, I may never have obtained any biological samples. I also want to thank Professor Sension for loaning us a copy of her former student's thesis to use as a reference when extracting and characterizing our samples.

I have a wonderful network of very close friends, most of whom live far away in 
places like Texas or Indiana. They keep me sane when I need to vent, give me places to visit when I have time for a vacation, and remind me that I can be graduate student and a human at the same time. I owe them many thanks for the phone calls, visits, and role-playing games over Skype.

I am lucky to have two of the greatest parents anyone could ever ask for. Ever since I was a small child, they nurtured my desire to learn and achieve. I never would have been able to get through a Ph.D. program if they hand't instilled in me a deep sense of value in striving not for perfection, but for excellence. They are the ones I turn to every time I need emotional support. I owe so much of what I am to them, and I hope that I make them proud, not just with my academic achievements but with the type of person I've become.

For Matt, who has supported me fiercely throughout this process and put up with far too much of my bellyaching, I am grateful. I hope I can repay the favor someday when it's time for him to go through graduate school. Until then, I hope my love and gratitude will suffice.

My whole family, including aunts, uncles, cousins and one very special grandma have always been supportive and interested in just what kind of project I happen to be working on and how it can make an impact on the world at large. They've nurtured my curiosity from the time I was a little kid playing with color-changing cars and performing "mutant studies" on teenage ninja turtles, and I'm lucky to have them all.

Finally, I wish to thank my whole committee for taking the time away from their own research to read this thesis, for discussions and guidance, and for helping me to succeed in this final stage of my education. 


\section{TABLE OF CONTENTS}

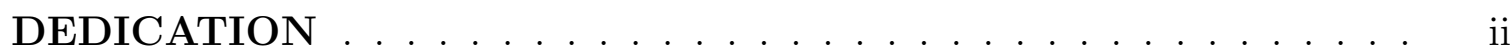

ACKNOWLEDGEMENTS . . . . . . . . . . . . . . iii

LIST OF FIGURES . . . . . . . . . . . . . . . . . . . . . . viii

LIST OF TABLES . . . . . . . . . . . . . . . . . . . . . . xii

LIST OF APPENDICES . . . . . . . . . . . . . . . . xiii

LIST OF ABBREVIATIONS . . . . . . . . . . . . . . . . xiv

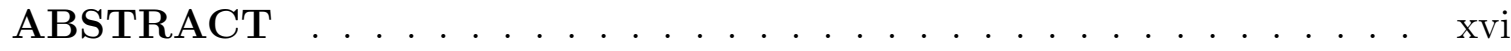

\section{CHAPTER}

I. Introduction . . . . . . . . . . . . . . . . . . . 1

1.1 Ultrafast Spectroscopy . . . . . . . . . . . . . . . . 2

1.2 Photosystem II . . . . . . . . . . . . . . . . . . . . . 5

1.3 Ultrafast Spectroscopy of the D1D2-cyt.b559 Reaction Center 13

1.4 Two-Dimensional Electronic Spectroscopy . . . . . . . . . . . 28

1.5 Scope and content of this work . . . . . . . . . . 33

II. Theory of $2 \mathrm{DES} \ldots \ldots \ldots \ldots \ldots$

2.1 Motivation: The Limitations of Linear Methods . . . . . . . . 47

2.2 Polarization and the density matrix . . . . . . . . . . 49

2.3 Double-sided Feynman Diagrams . . . . . . . . . . . . . . 54

2.4 Feynman Diagrams and the Density Matrix . . . . . . . . . 55

2.5 Types of Signal Contributions . . . . . . . . . . . . . . . 57

$2.62 \mathrm{D}$ spectra . . . . . . . . . . . . . . . . . . . . 59

2.7 A Note on Modelling . . . . . . . . . . . . . . 61

III. Experimental Methods . . . . . . . . . . . . . . . . . . . . . 70 
3.1 Laser Source . . . . . . . . . . . . . . . . . . . . . . . 70

3.2 NOPAs .......................... 71

3.3 Prism Compressor . . . . . . . . . . . . . . . . . . 74

3.4 Requirements for 2D Spectra . . . . . . . . . . . . 75

3.5 2DES with a Diffractive Optic . . . . . . . . . . . 75

3.6 2DES with a Pulse Shaper . . . . . . . . . . . . . . 84

3.7 Demonstration Experiments . . . . . . . . . . . . . . . . 91

3.8 Comparison of methods . . . . . . . . . . . . . . . . 102

IV. Photosystem II Studies _. . . . . . . . . . . . . . . . . . . 109

4.1 Experimental Methods . . . . . . . . . . . . . . . . . 110

4.2 Details of Data Acquisition . . . . . . . . . . . . . 117

4.3 Data Treatment . . . . . . . . . . . . . . . . . . 117

4.4 2D Spectral Features of the PSII RC [9] . . . . . . . . . 120

4.5 Kinetic Analysis . . . . . . . . . . . . . . . . . . . 122

4.6 Data Kinetics: 2D DAS of D1D2 data . . . . . . . . . . . 129

V. Conclusion and Future Work . . . . . . . . . . . . . . 147

APPENDICES . . . . . . . . . . . . . . . . . . . 154 


\section{LIST OF FIGURES}

\section{Figure}

1.1 A cartoon representation of the inner structure of a chloroplast, the center of photosynthesis light reactions in higher plants. . . . . . . 6

1.2 A structural model of the D1D2-cyt.b559 reaction center complex . 8

1.3 A spectrum of the D1D2-cyt.b559 reaction center at room temperature with main bands labeled. . . . . . . . . . . . . . . . . .

1.4 The crystal structures of (a) the BRC from $R$. Sphaeriodes and (b) the D1D2-cyt.b559 complex from spinach. . . . . . . . . . 14

1.5 Two example kinetic models for the RC from the literature. . . . . .

1.6 Graphical summary of exciton states included in the model of the RC by Novoderezhkin et al. . . . . . . . . . . . . . . . . . . .

1.7 A cartoon representation of a 2DES spectrum and associated energy level diagrams for a pair of coupled three-level systems. . . . . . . .

2.1 Double-sided Feynman diagrams for the rephasing and non-rephasing pathways . . . . . . . . . . . . . . .

2.2 A diagram of the density matrix elements involved during coherence and population times for a rephasing signal . . . . . . . . . . . 58

2.3 Energy levels diagrams for different coupling cases with cartoon representations of the 2DES spectra . . . . . . . . . . .

2.4 This flowchart describes the general process for modelling a 2DES spectrum on a given system.

3.1 Diagrams of NOPA beam geometry. . . . . . . . . . . . 
3.2 A series of experimentally obtained NOPA spectra, demonstrating the broad tunability of our apparatus. . . . . . . . . . . . . .

3.3 Experimental setup for 2DES with a diffractive optic and pulse sequence 79

$3.4 \quad$ A simulated spectral interferogram . . . . . . . . . . . . 80

3.5 A time domain trace of the inverse Fourier transform of a spectral interferogram (blue curve), including a DC contribution as well as the two well-separated interference terms. . . . . . . . . . . . . .

3.6 Real part of the zero-mean complex signal interference term after windowing in the time domain.

3.7 Diagram of the experimental setup for 2DES with a pulse shaper . . 86

3.8 Separation of rephasing and non-rephasing contributions for LDS750 in acetonitrile at $t_{2}=500 \mathrm{fs} \ldots \ldots \ldots$

3.9 2D spectra of rhodamine 800 in ethanol, showing different scatter subtraction schemes . . . . . . . . . . . . . . . . 99 90

3.10 A spectrum of the continuum pulse produced with a Sapphire window 92

3.11 Cartoon structure of a DNA-bound FRET pair. . . . . . . . . . . . 92

3.12 Absorbance (blue curve) and fluorescence (green curve) spectra of $\mathrm{Cy} 3$ and $\mathrm{Cy} 5$ (yellow and orange curves) from our duplexed DNA construct.

3.13 2D spectra of the DNA at $t_{2}=71$ ps showing the cross-peak indicative of energy transfer. . . . . . . . . . . . . . . . . . 99 95

3.14 Comparison of decay rates for $\mathrm{Cy} 3$ in the presence and absence of $\mathrm{Cy} 596$

3.15 Chemical structure of PERY . . . . . . . . . . . . . . 97

3.16 Absorption spectrum (black curve) of PERY showing the pump (green curve) and continuum (red curve) spectra. . . . . . . . . . . . . 97

3.17 Pump-probe data for PERY in DMSO . . . . . . . . . . . . . . 99

3.18 Absorptive 2D spectra of PERY in DMSO for four different waiting times, $t_{2} \ldots \ldots \ldots \ldots \ldots \ldots$ 
3.19 Ratio of diagonal to antidiagonal widths for the central peak (solid blue), lower peak (dashed red), and upper peak (dotted green), showing modulation with $\mathrm{a} \sim 240$ fs period. . . . . . . . . . . . . 101

$4.1 \quad$ A sample of PSII in a sample cell with untreated windows. . . . . . 111

4.2 Diagram of the complete cryostat system, including the attached pumps, flow controller, temperature controller, liquid nitrogen canister, and transfer tube. . . . . . . . . . . . . .

$4.3 \quad$ Spectra of pulses used in RC studies . . . . . . . . . . . . . . . . . 114

4.4 A comparison of uncorrected (a) and corrected (b) 2D spectra for the $\mathrm{RC}$ at $77 \mathrm{~K} \ldots \ldots \ldots \ldots \ldots$

4.5 A 2DES spectrum of the D1D2-cyt.6559 reaction center at $77 \mathrm{~K}$ demonstrating the spectral features at $t_{2}=32 \mathrm{fs}$

4.6 2D Data on the D1D2-cyt.b559 reaction center at $77 \mathrm{~K}$ at $t_{2}=215$ fs (top) and $t_{2}=603 \mathrm{fs}$ (bottom) . . . . . . . . . . 123

4.7 2D Data on the D1D2-cyt.b559 reaction center at $77 \mathrm{~K}$ at $t_{2}=1.5$ ps (top) and $t_{2}=100 \mathrm{ps}$ (bottom) . . . . . . . . . 124

$4.8 \quad \mathrm{R}^{2}$ values for the fits of the $\mathrm{RC}$ data to four exponentials. . . . . . . 129

4.9 2D DAS Component I showing kinetic processes for $t_{2}<400 \mathrm{fs}$. . 131

4.10 2D DAS Component II showing kinetic processes for 500 fs $<t_{2}<4$ ps132

4.11 2D DAS Component III showing kinetic processes for 4 ps $<t_{2}<$ $100 \mathrm{ps} \ldots \ldots \ldots \ldots \ldots \ldots$

4.12 2D DAS Component IV showing kinetic processes for 4 ps $<t_{2}<$

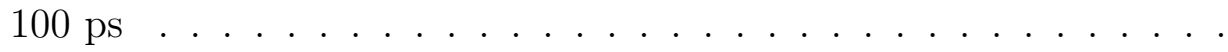

4.13 An excitonic energy-transfer model based on analysis of our data, using the model of Novoderezhkin et al. . . . . . . . . . . . . . . 140

A.1 2D Data on the reduced D1D2-cyt.b559 reaction center at $77 \mathrm{~K}$ at $t_{2}=30 \mathrm{fs} \ldots \ldots \ldots \ldots \ldots \ldots \ldots$

A.2 2D Data on the reduced D1D2-cyt.b559 reaction center at $77 \mathrm{~K}$ at $t_{2}=206 \mathrm{fs}$ (top) and $t_{2}=599 \mathrm{fs}$ (bottom) . . . . . . . 158 
A.3 2D Data on the reduced D1D2-cyt.b559 reaction center at $77 \mathrm{~K}$ at $t_{2}=1.5 \mathrm{ps}(\mathrm{top})$ and $t_{2}=100 \mathrm{ps}$ (bottom) . . . . . . 159

A.4 2D DAS spectra in the reduced D1D2-cyt.b559 reaction center at 77

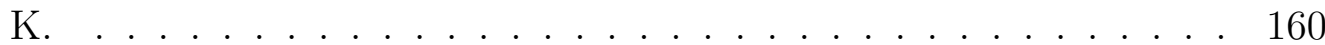

B.1 Covariance map between the upper and lower cross peaks located at each point and its conjugate across the diagonal. . . . . . . . . . 166

B.2 Coherence plot of the residuals from $\mathrm{RC}$ data for the upper diagonal (blue) and lower diagonal (green) cross peaks corresponding to potential coupling between excitons at $668 \mathrm{~nm}$ and $682.5 \mathrm{~nm}$. . . . 167

C.1 A diagram of the experimental setup for ZAP-SPIDER pulse characterization . . . . . . . . . . . . . . . 172

C.2 A ZAP-SPIDER interferogram . . . . . . . . . . . . 173

C.3 A retrieved spectrum (blue line) and phase (green line) of a nearly transform limited pulse (solid line) with a FWHM of $\sim 28$ fs. . . . . 175

C.4 A retrieved time domain profile of a nearly transform limited pulse (solid line) with a FWHM of $\sim 28$ fs. . . . . . . . . . . . . . . . 176

E.1 Design of a low-temperature, vacuum-rated sample cell using only compression fittings. . . . . . . . . . . . . . . . 193 


\section{LIST OF TABLES}

\section{$\underline{\text { Table }}$}

1.1 Summary of spectral bands of the RC . . . . . . . . . . . . 10

1.2 Major experiments on charge transfer . . . . . . . . . . . . . . 15

1.3 Major experiments on excitation energy transfer . . . . . . . . . 19

2.1 A comparison between 2D NMR and 2DES methods . . . . . . . 51

3.1 A comparison of two implementations of 2DES . . . . . . . . . 104

D.1 Stock solutions used in the PSII RC prep . . . . . . . . . . . 179

D.2 A list of recipes for all buffers used in the D1D2-cyt.6559 sample preparation. . . . . . . . . . . . . . . . 189 


\section{LIST OF APPENDICES}

\section{$\underline{\text { Appendix }}$}

A. Preliminary Data on Reduced RC Samples . . . . . . . . . . . . . . . 155

B. Electronic Coherences . . . . . . . . . . . . . . 165

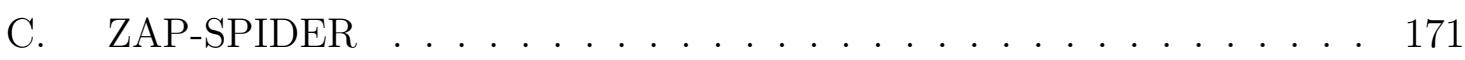

D. PSII Sample Preparation . . . . . . . . . . . . . . . 178

E. Sample Cell . . . . . . . . . . . . . . . . . . . 191 


\section{LIST OF ABBREVIATIONS}

2D DAS Two-dimensional decay associated spectra

2C2DES Two-color two-dimensional electronic spectroscopy

2DES Two-dimensional electronic spectroscopy

2DIR Two-dimensional infrared spectroscopy

2PE Two-pulse photon echo

BBO Beta-barium borate

BChl Bacteriochlorophyll

BRC Bacterial reaction center

CCD Charge-collecting device

CGS Common ground state

Chl Chlorophyll

CP Cross-peak

CS Charge separation

DAS Decay associated spectra

DMSO Dimethyl sulfoxide

DO Diffractive optic

DSP Digital signal processor

EET Excitation energy transfer

ESM Exponential series method

ESA Excited state absorption 
ESE Excited state emission

ET Energy transfer

FRET Fluorescence resonance energy transfer

GSB Ground state bleach

LHCII Light-harvesting complex II

LN Liquid nitrogen

LO Local oscillator

NMR Nuclear magnetic resonance

NOPA Non-collinear optical parametric amplifier

OD Optical density

PC Prism compressor

PERY $N, N^{\prime}$-bis (2,6-dimethylphenyl) perylene-3,4,9,10-tetracarboxylicdiimide

Pheo Pheophytin

PSI Photosystem I

PSII Photosystem II

RC D1D2-cyt.b559 reaction center complex

SFG Sum frequency generation

SHB Spectral hole burning

SNR Signal-to-noise ratio

SPM Self-phase modulation

SVD Singular value decomposition

TA Transient absorption

TRF Time-resolved fluorescence

ZAP-SPIDER Zero additional phase spectral interferometry for direct electric field reconstruction 


\begin{abstract}
Two-Dimensional Electronic Spectroscopy of the Photosystem II D1D2-cyt.6559 Reaction Center Complex

by

Jeffrey Allen Myers
\end{abstract}

Chair: Jennifer P. Ogilvie

Two-dimensional electronic spectroscopy (2DES) is a powerful new technique for examining the electronic and vibronic couplings and dynamics of chemical, semiconductor, and biological samples. We present several technical innovations in the implementation of 2DES. We have performed two-color 2DES experiments, extending the technique's ability to study energy transfer to states at frequencies far from the initial absorption. We have demonstrated 2DES in the pump-probe geometry using a pulse-shaper. This method eliminates many technical challenges inherent to previous implementations of 2DES, making it a more widely accessible technique. To broaden the available frequency information, we have demonstrated 2DES with a continuum probe pulse. We have utilized this method to observe vibrational wavepacket dynamics in a laser dye, demonstrating that these dynamics modulate 2D lineshapes and must be accounted for in modelling 2DES data.

We perform 2DES studies on the $\mathrm{Q}_{y}$ band of the D1D2-cyt.6559 reaction center of plant photosystem II. This reaction center is the core oxygen-evolving complex 
in plant photosynthesis, taking in light energy and forming a charge separated state capable of splitting water. Understanding the relationship between the structure and function has both fundamental importance and applications to improving artificial light-harvesting. Traditional spectroscopy methods have been unable to completely resolve the time-ordering of energy and charge transfer events or the degree of electronic coupling between chromophores due to severe spectral congestion in the $\mathrm{Q}_{y}$ band. 2DES extends previous methods by frequency-resolving an additional dimension to reveal the degree of static disorder and electronic coupling, as well as a detailed picture of energy and charge transfer dynamics that will allow tests of excitonic models of the reaction center. Our data show direct evidence of electronic coupling and rapid sub-ps energy transfer between "blue" and "red" states. We measure charge transfer times of 1-3 ps, with evidence to support a recent model in which primary charge separation follows two separate pathways. Slow time components of $\sim 7 \mathrm{ps}$ and $\sim 50$ ps are also observed. The former is consistent with slow energy transfer from blue-absorbing states while the latter may indicate secondary charge transfer or slow charge transfer from a degenerate trap state. 


\section{CHAPTER I}

\section{Introduction}

The rich variety of molecular machinery in nature is nothing short of staggering. Billions of years of evolutionary optimization have produced all the building blocks which make up the complex systems of life on earth. The building blocks are themselves complex marvels of efficient functionality - from the robust coding of DNA to molecular motors, the world of biology is one of many interesting systems and subsystems. Of particular interest to many researchers are the systems responsible for energy collection and storage. The vast majority of lifeforms on the planet obtain their energy either directly or indirectly (via secondary or tertiary consumption) from sunlight. Light-harvesting complexes in plants, algae and certain species of bacteria use a variety of pigments to collect light from much of the visible spectrum. Antenna complexes ensure a large absorption cross-section, but the energy must then be transferred to reaction center pigments which store the excitation in a more stable state of long-lived charge separation and, later, in chemical bonds. The photophysics of these systems is known to be extremely efficient, with a quantum efficiency greater than $95 \%$ in some cases [1]. In order to compete efficiently with other decay pathways and to prevent significant photobleaching and photodamage, the energy transfer must occur on very rapid time scales. Indeed, many of these photosynthetic complexes in-

clude mechanisms to reroute excitations or electrons as needed to prevent damage to 
the more sensitive sites [2].

The most advanced manmade photovoltaic technologies are currently obtaining efficiencies of $40-43 \%[3,4]$. While the chemical storage processes of natural photosynthesis are not especially efficient compared to the charge extraction of solar cells, the efficient photophysics of the initial absorption and charge separation steps may hold some guidance for the further development of artificial light-harvesting technologies. While there has been considerable research performed with the aim of producing artificial photosynthesis inspired by biological systems $[5,6,7]$, the mechanisms for efficient photochemical energy storage are not trivial. Biological light-harvesting systems likely gain their efficiency from the complicated arrangement of chromophores, leading to optimized coupling for efficient and nearly lossless funnelling of energy to the reaction centers. Whether there may be additional mechanisms at play, however, such as a more subtle quantum mechanical sampling of states through long-lived electronic coherences as some researchers have recently suggested $[8,9,10,11]$ remains an open question.

\section{$1.1 \quad$ Ultrafast Spectroscopy}

Finite temporal resolution places inherent limitations on the ability to study any dynamical system. One cannot hope to directly observe any process that occurs faster than the speed at which one can take a measurement. A basic tenet of information theory states that the highest frequency that can be resolved in a measurement is equal to half the sampling rate [12]. The consequences of attempting to measure processes faster that the resolvable limit of the apparatus include blurring (in photography, for instance), misinterpretation of the identification and number of observed processes, or averaging that may obscure not just the frequency but even the presence of an underlying oscillation. At the very least, the error bars on any measured rates are limited by the instrument response. 
In order to reach new temporal domains of study, new instruments must be produced. Consider, as a concrete example, two innovations in photography during the previous two centuries. In 1878, Eadweard Muybridge produced the first crisp, highresolution photographs of a galloping horse using snapshot photography, a method which eliminated the motion blur inherent to normal photography. This series of photographs was able to resolve a process on the millisecond scale. Harold Edgerton's famous stroboscopic picture of a bullet passing through an apple, taken several decades later, provides example of a further innovation in time-resolved measurements, progressing from the millisecond to the microsecond regime [13].

The study of dynamical processes in chemical and biological systems requires resolution of many orders of magnitude faster than the second. Short-lived chemical species in a reaction may only exist for milliseconds or microseconds, while protein motions and electronic relaxations occur on scales ranging over $10^{-8}-10^{-12}$ seconds. Proton transfer and charge separation, which are crucial events in many biological processes, occur on a picosecond $\left(10^{-12} \mathrm{~s}\right)$ time scale. Vibrational motion and fast electronic energy transfer can occur even faster, on the femtosecond $\left(10^{-15} \mathrm{~s}\right)$ scale $[13,14]$.

Time-resolved studies of these processes and many others have deepened and expanded our understanding of molecular dynamics, transforming chemistry and biology into the rich and complex fields we know today and promising many exciting directions for the progression of knowledge in the years to come. This has only been possible with a series of rapid innovations in temporal resolution beginning in the middle of the twentieth century and culminating in the ultrafast pulsed laser, the only device capable of accessing the most rapid of these events.

The first short-lived intermediates in chemical reactions were viewed by Porter and Norrish in 1949 with the use of flash photolysis $[15,16]$. This type of experiment, using an incoherent source such as a Xenon flashlamp, could achieve resolution in the 
microsecond regime. Fluorescence studies and time-resolved absorption continued on this order of temporal resolution until the early 1960s, when the invention of the Qswitched laser allowed researchers to achieve nanosecond resolution [17]. The advent of mode-locked laser technology granted scientists another three orders of temporal resolution, reaching into the picosecond regime, and opened up the age of ultrafast optics, a term which generally refers to time scales below $10^{-9} \mathrm{~s}[18,19]$. Subsequent innovations led to the generation of femtosecond laser pulses in the 1980s [20], and today any researcher with adequate funding can purchase a commercial laser system capable of producing visible or infrared pulses of 10-100 fs duration. The current frontier of ultrafast optics is now in the attosecond regime, where x-ray pulses of $\sim 10^{-16}$ s can be used to directly study electron orbitals [21, 22, 23].

The power of ultrafast optics comes into play in the new field of ultrafast spectroscopy. Students of modern physics learn that atoms and molecules exist in a set of discrete quantum mechanical energy states which are defined by the arrangement and magnitude of the constituent masses and charges. The basis set of electronic transitions is further complicated by vibrational degrees of freedom, which expand the manifold of states. In large systems such as photosynthetic proteins, the manifold of states and interplay between interacting molecules is complex, and the understanding of the electronic structure and how it relates to function is an active area of theoretical and experimental research $[24,25]$.

The transitions between electronic states involve absorption or emission of photons, or from a wave standpoint, resonance with an externally oscillating electromagnetic field. In a complicated system, the set of resonance frequencies provide both a way to identify the species involved and a method for probing the underlying energetic dynamics inherent to the functioning of the system. Spectroscopy, as a discipline, is the study of this interaction between light and matter as a function of the optical frequencies involved. Linear spectroscopy methods are only able to probe the man- 
ifold of first excited states and cannot detect energy transfer or resolve dynamical processes. Techniques in nonlinear spectroscopy, however, can explore higher excited states and directly monitor dynamics on an ultrafast time scale.

\subsection{Photosystem II}

The process of photosynthesis in organisms such as plants and certain types of bacteria is fascinating and complex. Within the cells of higher plants, photosynthesis occurs in organelles called chloroplasts. Each chloroplast acts as an energy center for the cell, collecting sunlight in order to fuel biochemical reactions that store energy in the chemical bonds of sugars, carbohydrates and other metastable substances. These can be easily ported to other organelles or cells for metabolism or storage as needed.

Each chloroplast is filled with a fluid solution called the stroma and contains a large, folded vesicle or series of vesicles called the thylakoid. The thylakoid is folded into cylindrical stacks of disklike sacs called grana, which are connected to each other with unstacked stromal lamellae (see Figure 1.1). The thylakoid membrane contains the three main physical components of the photosynthesis light reactions: Photosystem I (PSI), which is primarily located in the stromal lamellae, Photosystem II (PSII), which is found almost exclusively in the stacked grana, and the cytochrome $b_{6} f$ complex, which is found throughout the membrane [26].

Briefly, the process of photosynthesis proceeds when PSII absorbs photons and produces a charge-separated state capable of splitting $\mathrm{H}_{2} \mathrm{O}$. An antenna structure known as light-harvesting complex II (LHCII) contains both chlorophyll $a$ and chlorophyll $b$ chromophores, as well as carotenoids, which gives it a broad absorption spectrum capable of collecting light at many frequencies on the blue side of the spectrum and funnelling energy downhill to the reaction center. The transmembrane proteins CP43 and CP47 are more closely bound to the core reaction center and pass excitations to the central chromophores involved in primary charge separation. The 


\section{Chloroplast}

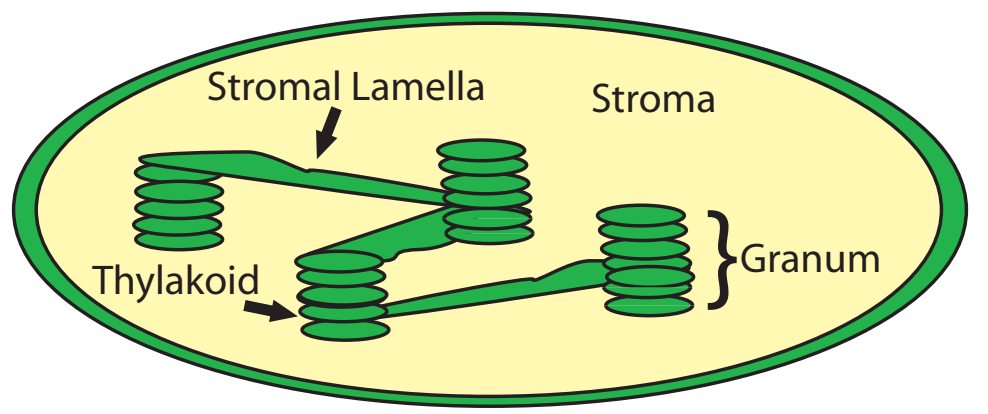

Figure 1.1: A cartoon representation of the inner structure of a chloroplast, the center of photosynthesis light reactions in higher plants.

electrons extracted after two absorption events doubly reduce a quinone molecule $\left(\mathrm{Q}_{B}\right)$ near the stromal surface. $\mathrm{Q}_{B}^{2-}$ diffuses through the membrane with two attached hydrogen ions as $\mathrm{Q}_{B} \mathrm{H}_{2}$ to the cytochrome $b_{6} f$ complex, which is responsible for pumping protons across the thylakoid membrane to produce a $\mathrm{pH}$ gradient [1]. PSI accepts water-extracted electrons from PSII and uses these in conjunction with electrons from another absorption-driven charge transfer event to produce the intermediate electron acceptor NADPH [26]. Carbohydrates are produced in the dark reactions via the Calvin cycle.

The many pathways and complex cycles of charge transfer and chemical synthesis involved in photosynthesis are the subject of a great deal of research which could fill several volumes. The biological focus of this thesis covers the photophysics and primary charge separation in the smallest subsystem of the PSII machinery: the reaction center complex.

\section{The D1D2-cyt.b559 Reaction Center Complex}

The core oxygen-evolving complex of Photosystem II and the focus of this work is the D1D2-cyt.b559 reaction center complex (RC). From biochemical studies, we now know that the RC complex is composed of six chlorophyll $a$ (Chl $a$ ), two pheophytin 
$a$ (Pheo $a)$, and one or two $\beta$-carotene molecules $[27,28]$, as shown in Figure 1.2. Note that while the quinones $\left(\mathrm{Q}_{A}\right.$ and $\left.\mathrm{Q}_{B}\right)$ are shown for illustrative purposes, these molecules are removed from our samples during the extraction process, as are CP47 and CP43. This means that the complete water-splitting process is not achieved in these samples. However, studies of more complete machinery suggest that the initial steps of energy transfer and charge separation are largely preserved in these simplified systems $[29,30]$. By studying the simplified system, we avoid many of the difficulties in measurement and interpretation of data that can arise when studying the larger complexes. Due to the similar chromophore content of CP43, CP47 and the core RC, the antennas have largely overlapping absorption spectra with the core RC, which introduces a great deal of complexity to spectroscopic studies.

\section{Spectral Features}

The absorption spectrum of the RC is divided into several bands, shown in Figure 1.3. The major spectral bands of the $\mathrm{RC}$ are summarized in Table 1.1. The $\mathrm{Q}_{x}$ and $\mathrm{Q}_{y}$ bands are both due to the $\pi-\pi^{*}$ molecular transition of the Chl a molecules. The separation between the bands is due to an asymmetry in the Chl $a$ molecule [31]. One of the challenges inherent in studying the $\mathrm{RC} \mathrm{Q}_{y}$ band is the spectral congestion involved in all the overlapping transitions. The $\mathrm{Q}_{y}$ band of unbound Chl $a$ or Pheo $a$ in solution has a bandwidth of $\sim 400 \mathrm{~cm}^{-1}$. Electronic coupling between the pigments and differences in local solvent or protein environment shifts these levels slightly within the RC, so the total $\mathrm{Q}_{y}$ band covers a width of $550 \mathrm{~cm}^{-1}$ [32], which does not change significantly with temperature, though the spectra of individual transitions will narrow at low $\mathrm{T}[33,34]$. With eight coupled chromophores and the addition of possible charge transfer states producing an even greater number of exciton states within the $550 \mathrm{~cm}^{-1}$ bandwidth, the severe congestion obscures and complicates any analysis of the dynamics underlying the broad lineshape. The $\mathrm{Q}_{y}$ band is of particular 


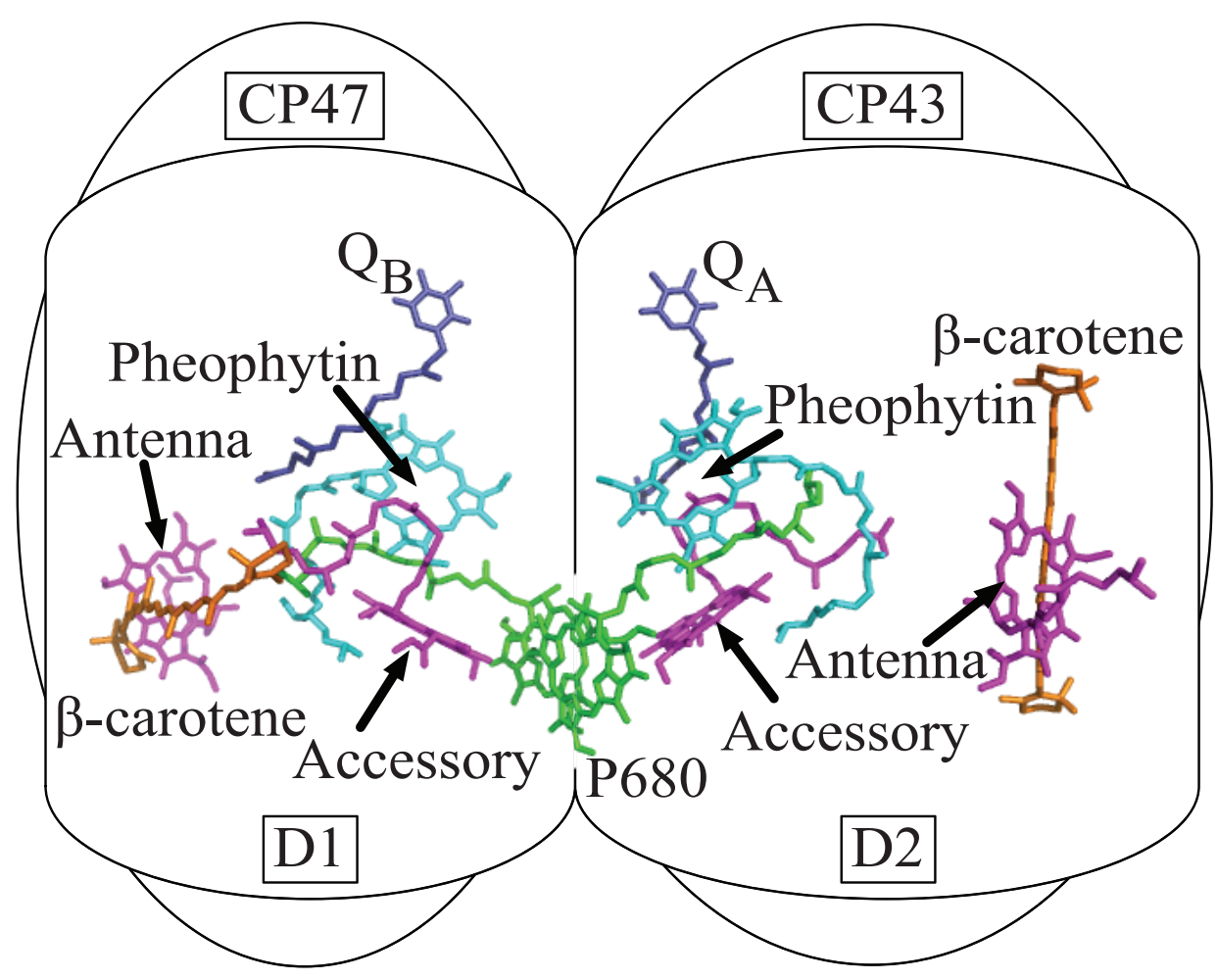

Figure 1.2: A structural model of the D1D2-cyt. 6559 reaction center complex. The quinones $\left(\mathrm{Q}_{A}\right.$ and $\left.\mathrm{Q}_{B}\right)$ and antenna complexes CP47 and CP43 are not present in our samples but are shown here as part of the larger structure. 
interest to researchers, however, as these are the transitions involved most directly in excitation energy transfer (EET) and charge separation (CS) in the RC. Due to the severe spectral congestion of the $\mathrm{Q}_{y}$ band, understanding its underlying structure is an ongoing effort.

The pheophytin $\mathrm{Q}_{x}$ band, near $544 \mathrm{~nm}$, is of interest primarily as a direct probe of the primary charge separation, but it contains no direct spectroscopic information on the chlorophylls and is therefore not able to probe the EET events in the complex as a whole. Pheo $a$ molecules are structurally similar to Chl $a$, the primary difference being that the Pheo a lacks a $\mathrm{Mg}^{2+}$ ion in the center of the porphyrin ring that is present in Chl a. This does not affect the spectral properties of the $\mathrm{Q}_{y}$ band significantly, but it does provide the Pheo $a$ with a distinct $\mathrm{Q}_{x}$ band. This feature is useful for detecting the reduced Pheo $a$ state (Pheo $a^{-}$) that occurs following charge separation. Complications arise from the fact that this bleach band cannot by itself distinguish between the first excited state (Pheo $\left.a^{*}\right)$ and the anion state (Pheo $a^{-}$) $[29]$.

Another noteworthy feature of the spectrum is the Soret region (or B-band) in the blue part of the spectrum. This band is most useful for characterization of the $\mathrm{RC}$ sample purity, as the ratio of the two Soret peaks serves as a measure of pigment stoichiometry and can be used to differentiate a pure RC sample from a sample in which antenna complexes are still attached, such as RC-CP47, or free antennas which act as contaminants in RC studies [35].

\section{Comparison to the bacteria reaction center}

Because of their structural similarity, the $\mathrm{RC}$ is often compared to the bacterial reaction center $(\mathrm{BRC})$ from photosynthetic bacteria $[36,37]$, the structure and kinetics of which were thoroughly studied and fairly well understood at a time when the plant RC was first isolated for study. While this comparison is useful as a first 


\begin{tabular}{|c|c|c|}
\hline Wavelength & Feature & Comments \\
\hline $400-450 \mathrm{~nm}$ & Soret region & $\begin{array}{l}\text { Used for characterization of sample } \\
\text { via pigment stoichiometry. }\end{array}$ \\
\hline $460 \mathrm{~nm}$ & $\mathrm{P} 680^{+}$and Pheo $a^{-}$ion bands & $\begin{array}{l}\text { Overlaps excited state absorption of } \\
\text { P } 680^{*} \text {. Not probed in these studies. }\end{array}$ \\
\hline $544 \mathrm{~nm}$ & Pheo $a \mathrm{Q}_{x}$ band bleach & $\begin{array}{l}\text { Used to confirm Pheo } a \text { reduction in } \\
\text { dithionite experiments. In general, } \\
\text { cannot distinguish between Pheo } a^{-} \\
\text {and Pheo } a^{*}\end{array}$ \\
\hline 660-695 nm & $\begin{array}{l}\mathrm{Q}_{y} \text { band, containing absorption } \\
\text { of all Chl } a \text { and Pheo } a \text { pigments }\end{array}$ & $\begin{array}{l}\text { Severe spectral congestion makes } \\
\text { interpretation of data difficult. Main } \\
\text { focus of this work }\end{array}$ \\
\hline $735 \mathrm{~nm}$ & $\begin{array}{l}\text { Qy stimulated emission sideband } \\
\text { of Chl } a \text { and Pheo } a \text { pigments }\end{array}$ & $\begin{array}{l}\text { Weak feature. Not probed in these } \\
\text { studies. }\end{array}$ \\
\hline $790-820 \mathrm{~nm}$ & $\mathrm{P}_{680^{+}}$and Pheo $a^{-}$ion bands & $\begin{array}{l}\text { Overlaps excited state absorption of } \\
\text { P } 680^{*} \text {. Not probed in these studies. }\end{array}$ \\
\hline
\end{tabular}

Table 1.1: Summary of spectral bands of the RC 


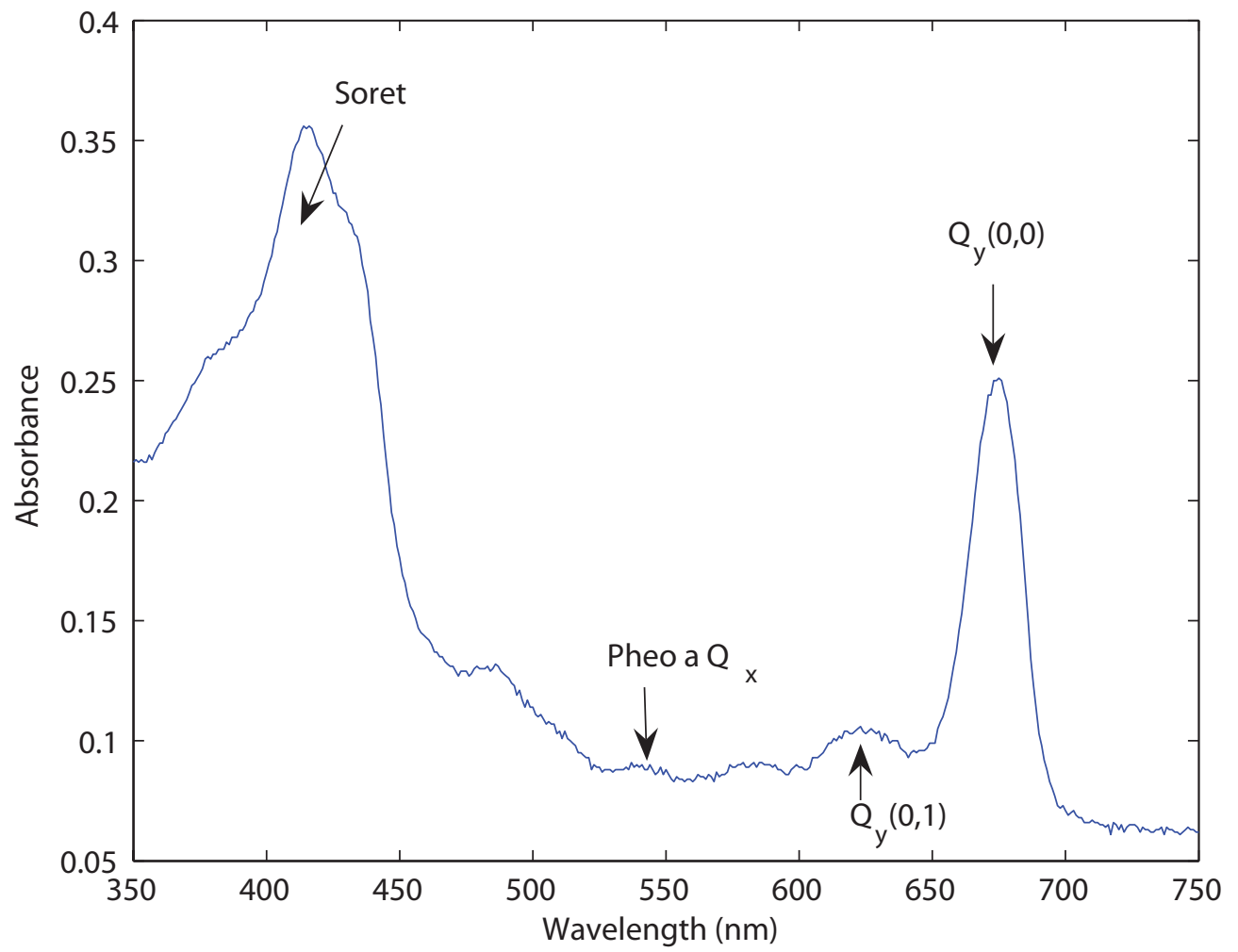

Figure 1.3: A spectrum of the D1D2-cyt.6559 reaction center at room temperature with main bands labeled. 
approximation for a kinetic scheme, there are nevertheless some important distinctions which motivate a more unique treatment of the RC. The BRC contains a special pair of closely spaced bacteriochlorophyll (BChl) molecules with nearly parallel dipole moments at a close separation of $\sim 7.6-8.0 \AA$, depending on the species [32]. The coupling between this dimer of BChl is sufficiently strong to shift its absorption band far enough to the red to produce an excitation "trap" state, a lower energy state that captures an excitation with a low probability of reversal due to the energy barrier. This pair of BChl is referred to as the "special pair" and acts as primary electron donor in the BRC [32].

The analogous Chl $a$ molecules in the structure of the RC are often referred to as P680, a potentially misleading name that suggests a strongly-coupled dimer. From previous crystal structure data of the plant RC, the separation between the Chl $a$ molecules of P680 was until recently thought to be much larger than in the BRC special pair, on the order of $10 \AA$ [38]. This was supported by the spectroscopic observation that P680 does not have a shifted $\mathrm{Q}_{y}$ band absorption, as one would expect from a strongly coupled dimer. The BRC special pair, in stark contrast, shows an energy shift of $700-800 \mathrm{~cm}^{-1}$ in $R$. sphaeroides and $1500-1700 \mathrm{~cm}^{-1}$ in B. viridis, resulting in a distinctly red-shifted absorption peak. Recent high resolution crystal structure data on the RC, however, indicate a shorter center-to-center separation in the P680 pigments, on the order of 8.1-8.3 $\AA$, comparable to the BRC [39]. From this, one would expect a stronger excitonic coupling, resulting in a special pair for PSII. The absorption spectrum does not support this, however. The spectrum of the $\mathrm{RC}$ is highly congested, and there is no evidence for a spectral shift greater than 300 $\mathrm{cm}^{-1}$. The energy levels may also be further influenced by couplings between the P680 pigments and other closely spaced reaction center chromophores. This has led many researchers to adopt a multimer model to describe the couplings and dynamics of the $\mathrm{RC}[37,40,41,42]$. As this kind of model gains popularity, the nomenclature is 
changing to reflect the shift in thought. The name "P680" was originally chosen for the pair of chlorophylls because researchers saw a structure similar to the bacterial special pair and assumed it would act as a dimer. Recent papers are now referring to the chromophores separately as $\mathrm{P}_{D 1}$ and $\mathrm{P}_{D 2}$, respectively [25, 43]. The crystal structures of the BRC and RC are shown in Figure 1.4.

\subsection{Ultrafast Spectroscopy of the D1D2-cyt.b559 Reaction Center}

The RC has been studied with many different spectroscopic techniques over the last 23 years since its initial purification in 1987 [44]. From these have emerged a number of kinetic schemes, including rates for EET and CS within the samples and assignments for the primary electron donor. Major results are summarized in Tables 1.2 and 1.3, adapted from the reviews of Greenfield and Wasielewski [29] and Yoder, Cole and Sension [32] and expanded to include subsequent research.

The most prevalent spectroscopic techniques used in the study of the $\mathrm{RC}$ are each described in the following subsections, including historical notes and descriptions of the strengths and limitations of each technique. This should not be taken as a criticism on the excellent work done thus far but as a motivation for the need to further characterize this fascinating system with powerful, emerging spectroscopic techniques, such as two-dimensional electronic spectroscopy (2DES), which is the subject of this thesis. The data presented in Chapter 3.8 are the first reported results of a 2DES study on the D1D2-cyt.b559 reaction center. 
(a) BRC (R. Sphaeroides)
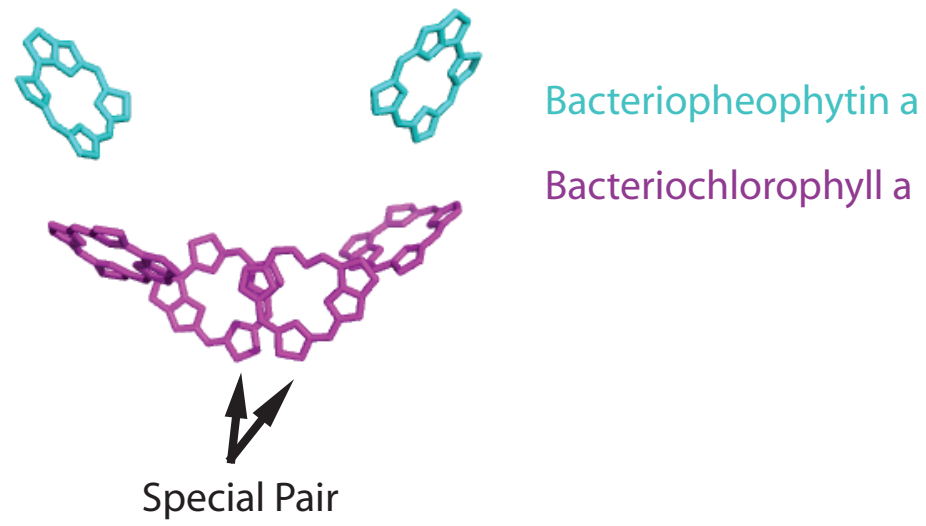

Bacteriochlorophyll a

(b) D1D2 (spinach)

Pheophytin a

Chlorophyll a

的
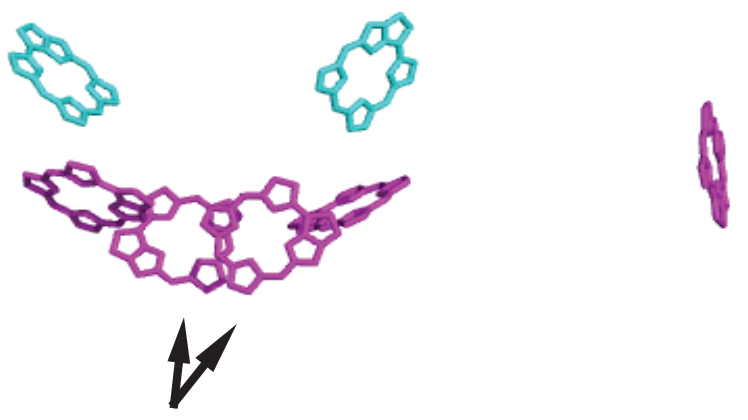

P680

Figure 1.4: The crystal structures of (a) the BRC from $R$. Sphaeriodes and (b) the D1D2-cyt.b559 complex from spinach. The peripheral chlorophylls on the outer extremes of the RC structure are unique to plant reaction centers. 


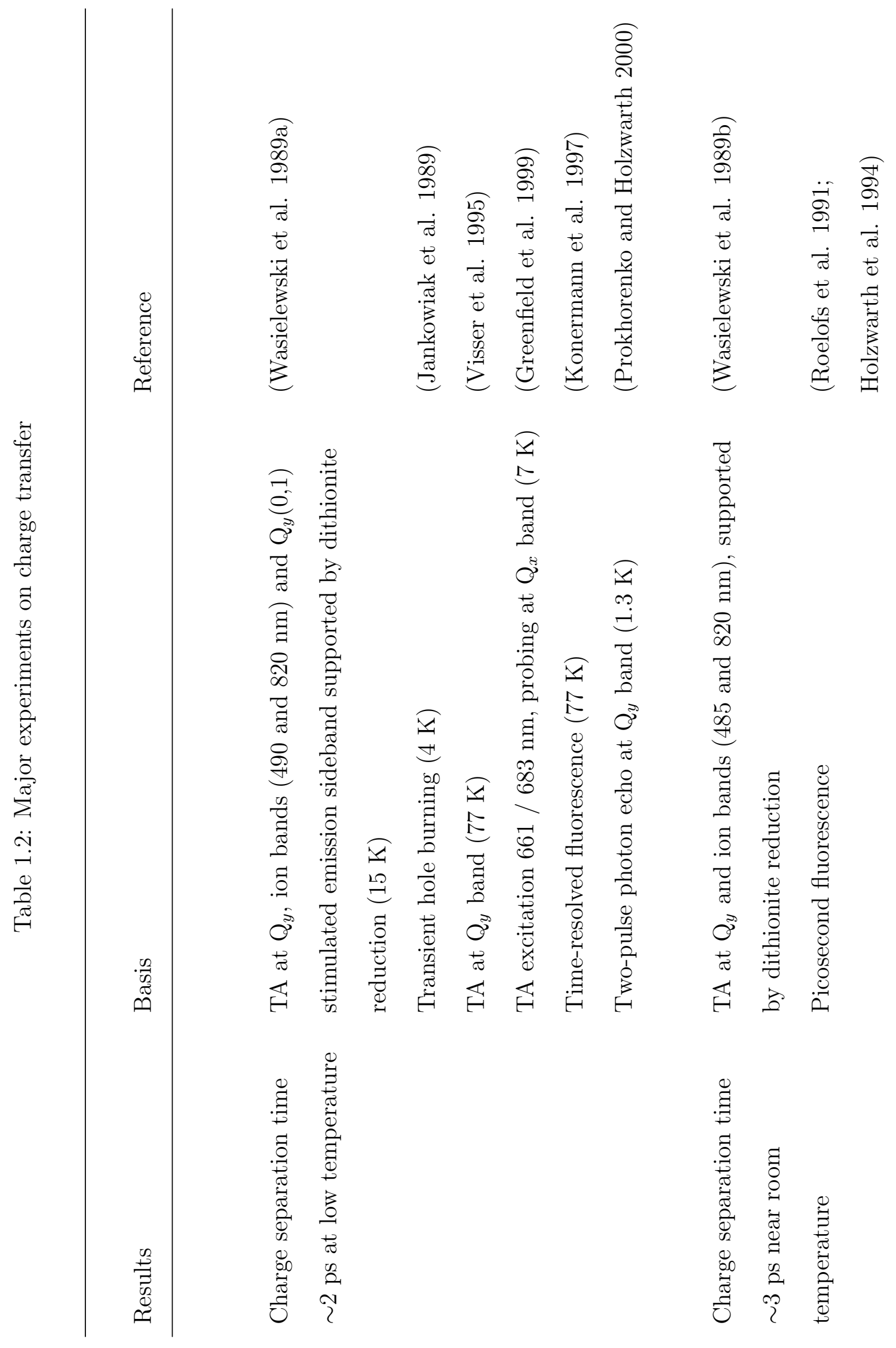




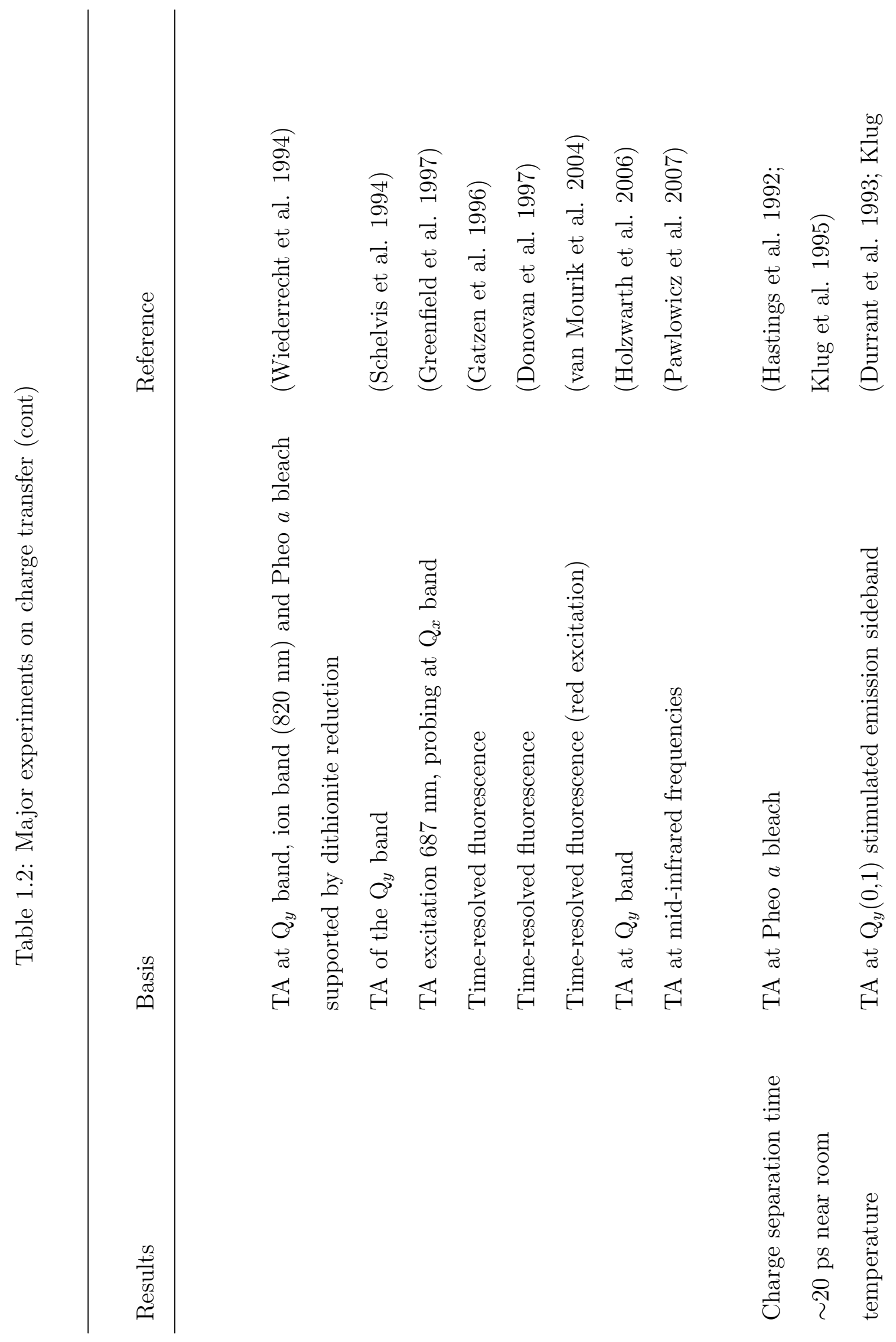




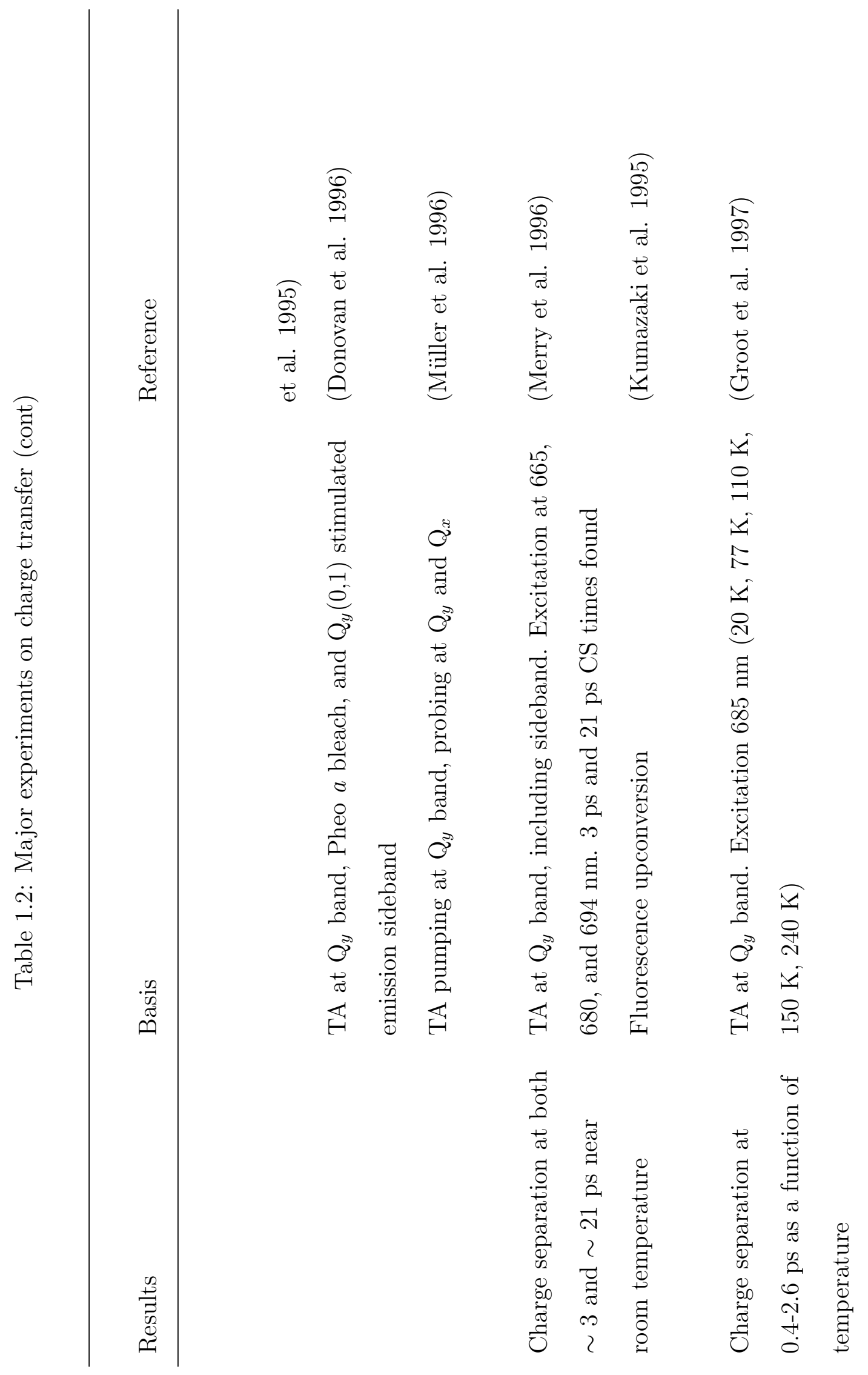




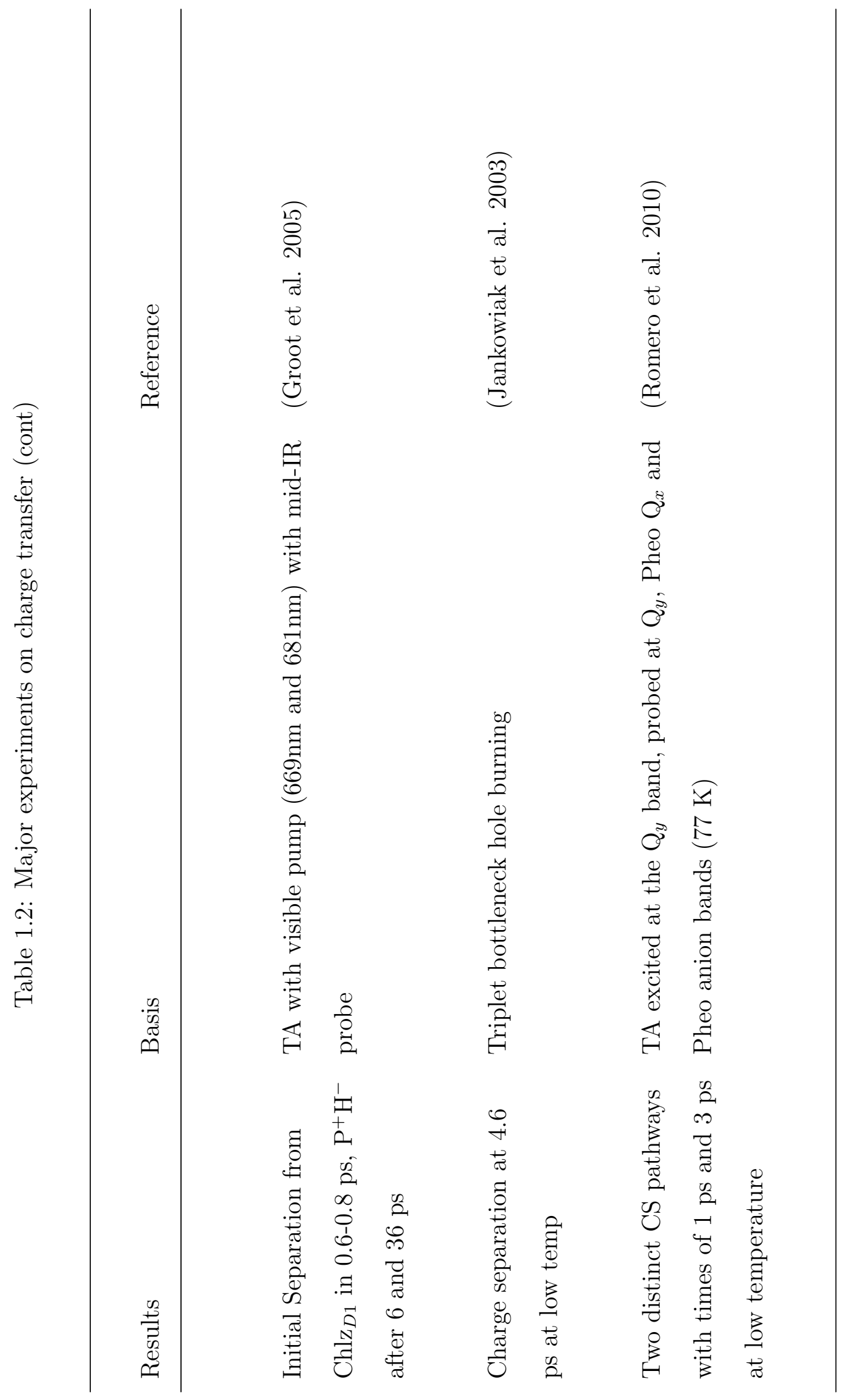




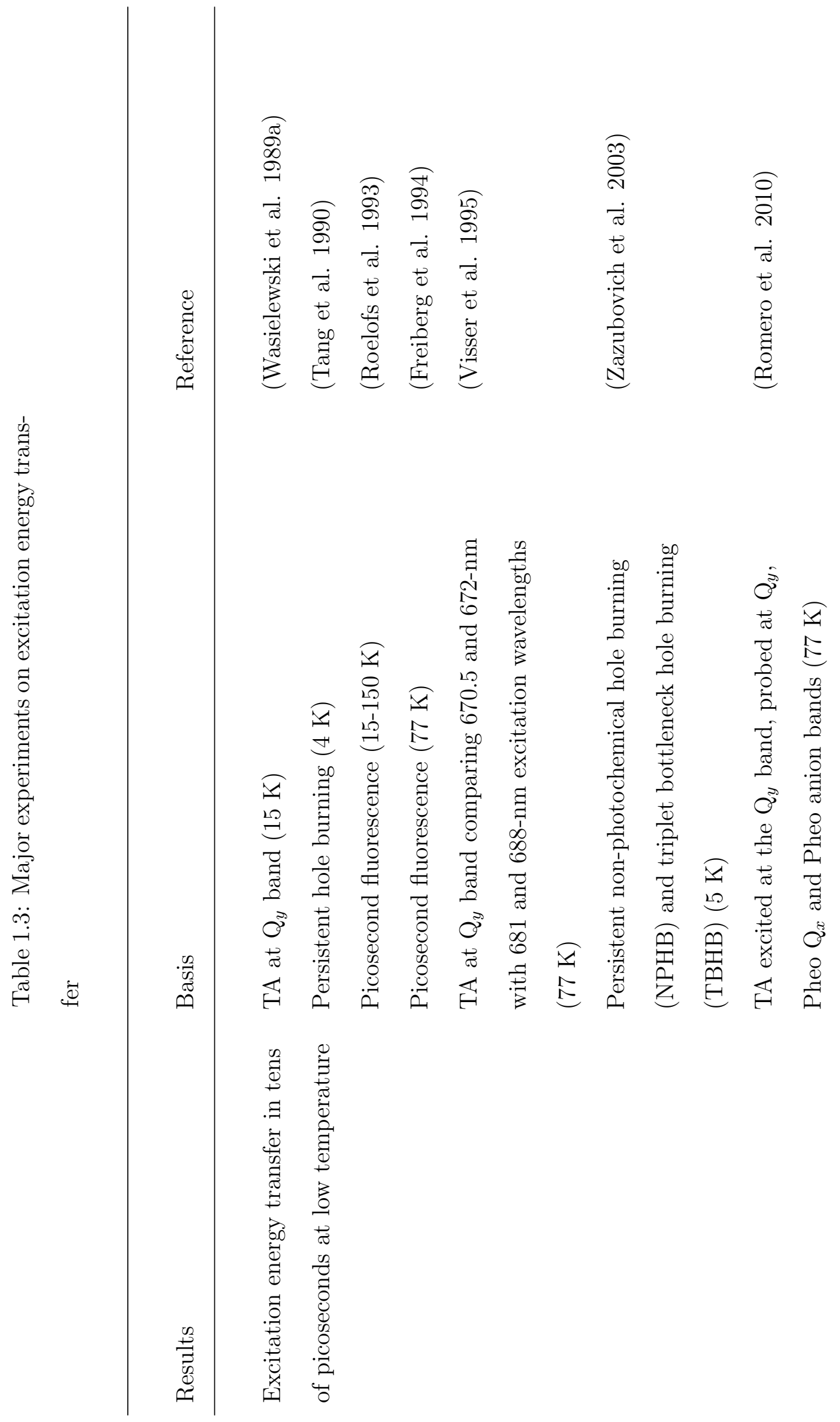




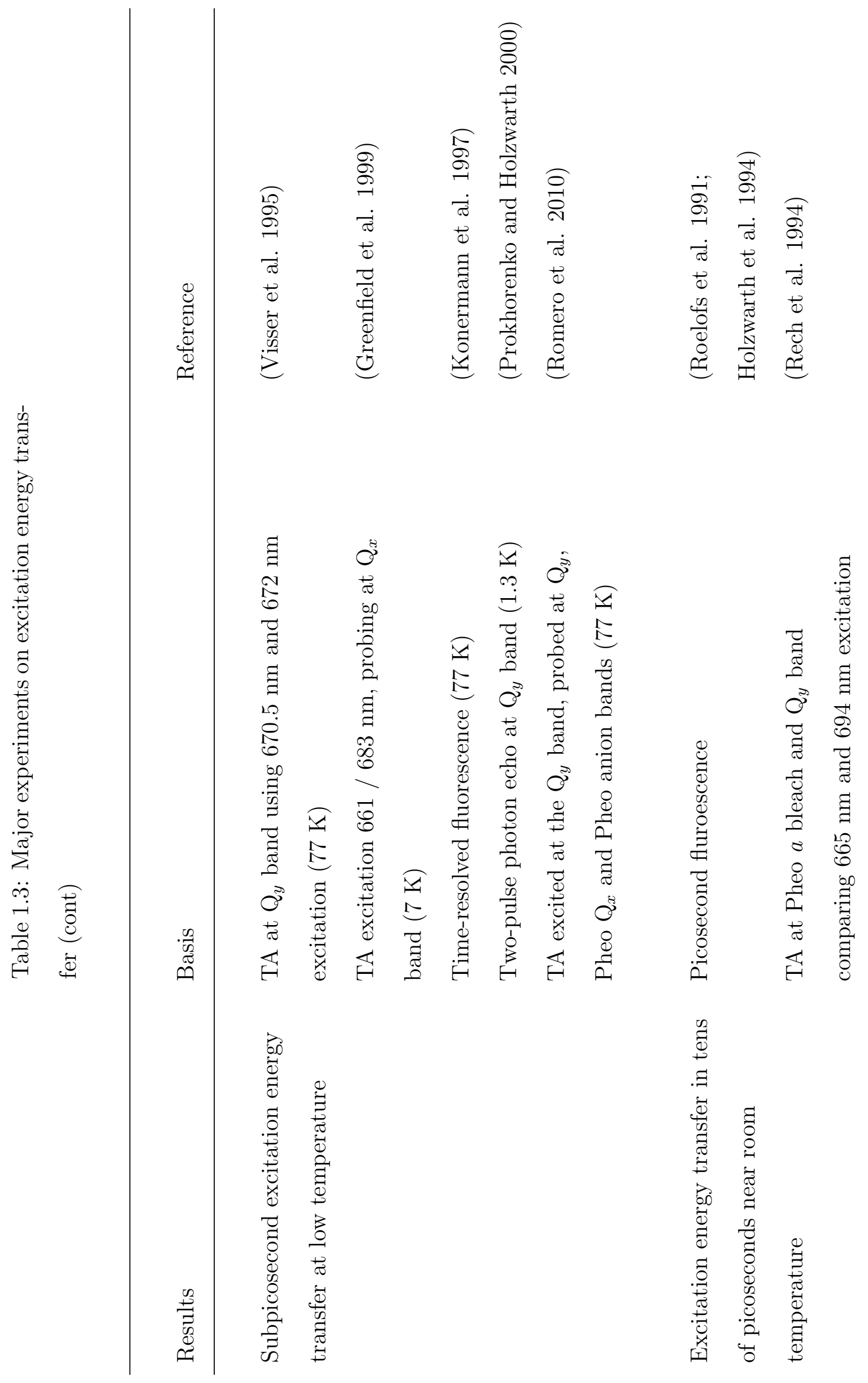




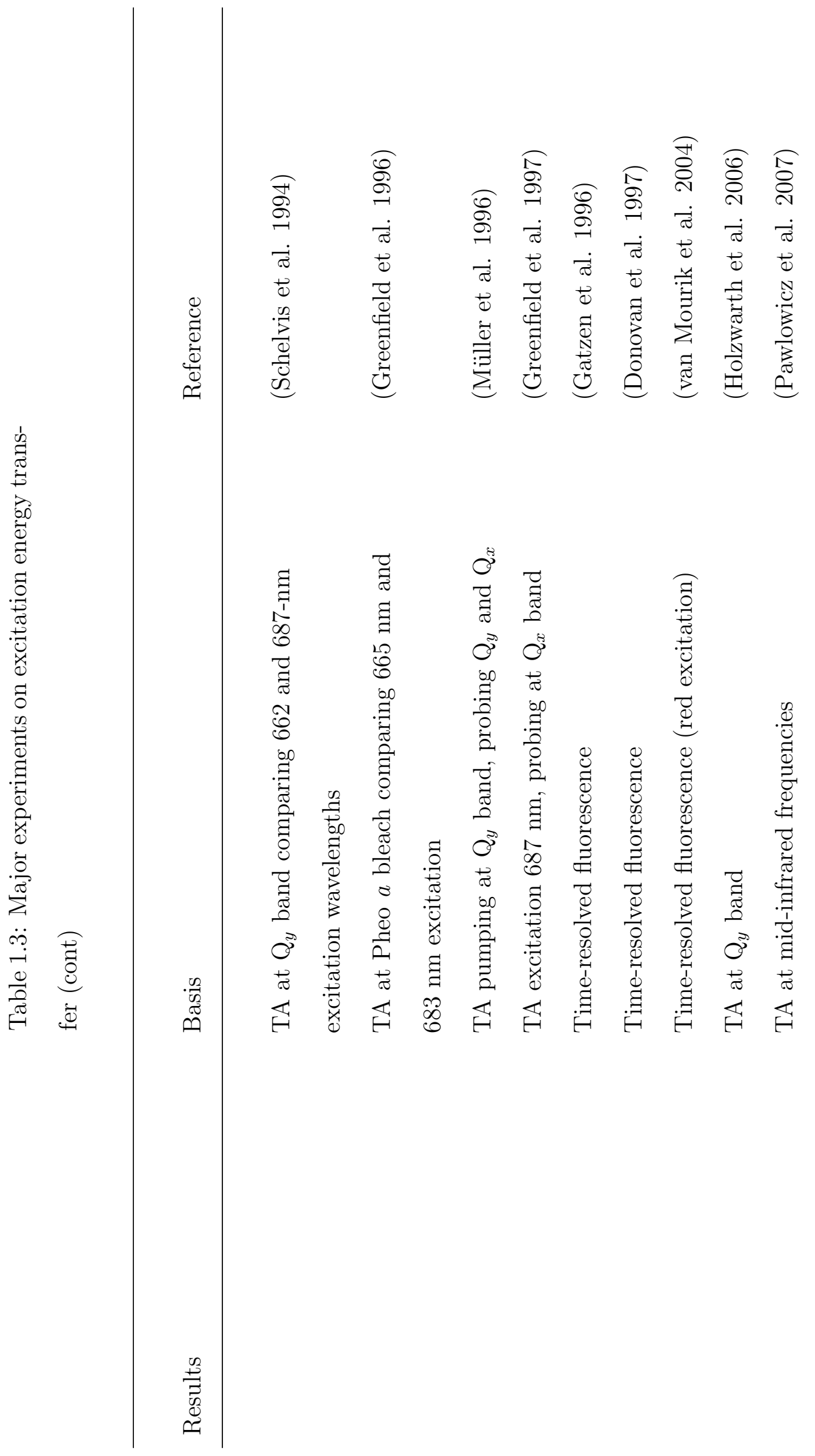




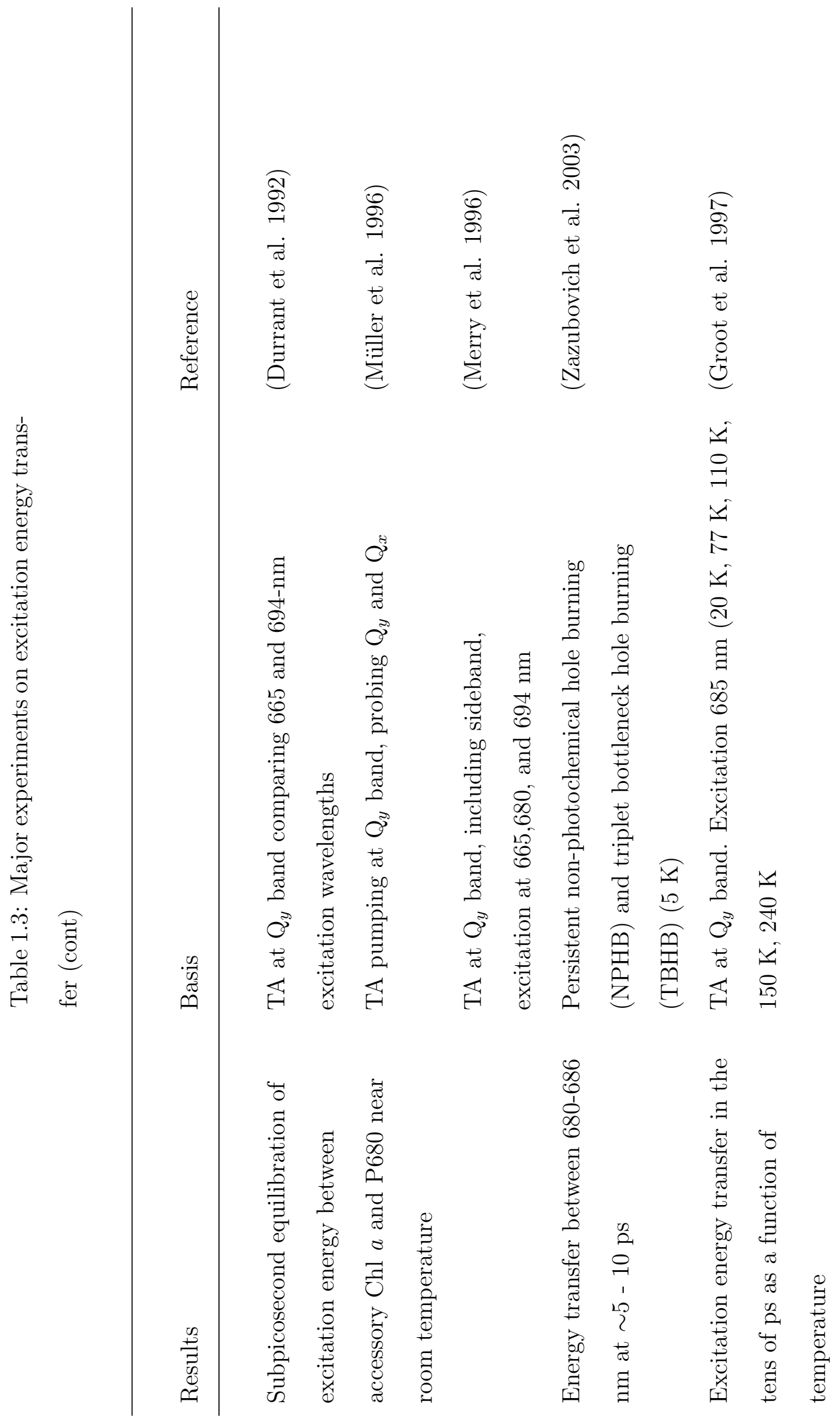




\section{Time-Resolved Fluorescence}

Time-resolved fluorescence (TRF) techniques have been used by a few groups, notably the Sension and Holzwarth labs [45, 46, 47, 48, 49, 50, 51]. In these experiments, it is typical to excite the sample with a picosecond pulse and detect the resulting fluorescence with a photomultiplier or streak camera. The main advantage of this technique is the inherently high signal-to-noise ratio (SNR). A major drawback, however, is that the time resolution is generally poor. Instrument responses may be as poor as $30 \mathrm{ps}$, though in this technique it is possible to partially deconvolve the instrument response to achieve better resolution [29]. An experimental innovation by Donovan et al. using a streak camera was able to achieve an instrument response of 4.5 ps [50]. Early TRF experiments reported charge separation times in the tens of ps near room temperature, but more recent studies tend to place this value closer to $1-3$ ps $[49,50,52]$.

There has been one experiment performed with the technique of fluorescence upconversion [53]. While this technique boasts increased time resolution and was lauded as a needed technique by Greenfield and Wasielewski in their 1996 review paper [29], it has been pointed out that this technique suffers from complications because its high photon fluency is likely to multiply excite the reaction centers [32].

\section{Spectral Hole Burning}

Spectral hole burning (SHB) is a frequency domain spectroscopic method by which a sample at very low temperature has a hole burned into its absorption spectrum at a specific frequency. The population dynamics are probed by a second beam which examines the spectral recovery of the hole after some delay.

Most of the SHB studies to date on the RC have been performed by Small and coworkers $[33,54,55,56,57,58,59]$. SHB has several advantages over other techniques: the measurements at low temperature are more likely to give intrinsic rates, 
since the transfer processes are largely unidirectional. Intrinsic rates are single-process rates uncoupled from complications due to reversibility of the transition or rate limitation from a cascade of sequential processes. Also, the ability to differentiate between transient and persistent holes gives an extra degree of selectivity [29]. The most severe drawback of this technique is that it can only be used at temperatures of a few Kelvin. At higher temperatures, the spectra are dominated by dephasing effects, so it is impossible to measure temperature dependence or gain a better sense of the transfer rates near the native temperatures of the systems. The SHB experiments above have generally given low-temperature CS rates of 2-6 ps and EET rates of 5-10 ps and in the tens of ps.

\section{Two-pulse Photon Echo}

A handful of two-pulse photon echo (2PE) studies have been performed on the $\mathrm{RC}$ [57]. The 2PE technique is the time-domain analogue of SHB. The earliest experiments were performed using an accumulated photon echo technique [60, 61], which can suffer from reliance on a long-lived intermediate triplet state [62]. One advantage to $2 \mathrm{PE}$ studies is that the signals are due to effects which destroy the coherence and are therfore insensitive to reversible processes involved in population dynamics, which is not the case in TA experiments [62]. The weakness of this technique, however, is its inability to directly probe energy transfer or excited state absorption. While one study resolved no kinetics faster than 100 ps [60], the others found an intrinsic charge separation time of $\sim 1.5$ ps at low temperature, and Prokhorenko and Holzwarth detected a sub-ps energy transfer process [62] that was later disputed by SHB experiments [57]. 


\section{Transient Absorption}

One of the most widely used techniques has been transient absorption (TA) spectroscopy, which has the advantages of being applicable to the study of the kinetics of energy and charge transfer at all temperatures. The earliest studies were performed with parallel beam polarization $[63,64,65,66,67,68,69,70,71,72]$, though criticism that the measured decay rates had been affected by anisotropy effects quickly led to a prevalence of magic angle polarization in these experiments $[47,73,74,75,76,77,40]$. In order to better elucidate the dynamics of P680, some researchers have attempted to excite the sample at the far red tail of the absorption band in order to selectively excite a subpopulation consisting of only a few pigments [67, 74, 69, 65, 70, 47, 78]. Due to the high degree of degeneracy between chromophores and the evidence of a rapid sub-picosecond equilibration between "blue" and "red" pigments, however, there is some disagreement as to the fruitfulness of selective excitation as a method for identifying subpopulations [79]. Some experiments have attempted to clarify the kinetics by probing different parts of the spectrum or taking measurements at various temperatures $[75,80,81,82,30,83,43]$. However, the results of numerous TA experiments on the RC have sometimes been conflicting between researchers, particularly in the case of different temperatures and excitation conditions.

While TA has the ability to selectively excite at a specific frequency and to probe a broad bandwidth, this comes at the expense of time resolution. The uncertainty relationships between time and bandwidth mean that the more selective (narrowband) the excitation pulse, the longer the pulse duration. As an example, a pulse near 680 $\mathrm{nm}$, in the $\mathrm{RC}_{y}$ band, with a bandwidth of $5 \mathrm{~nm}$ would be unable to resolve sub-100 fs kinetics. This inherent tradeoff is a limitation in TA studies. The 2DES technique used in this thesis circumvents this limitation, providing simultaneously high time and frequency resolution. 


\section{Open Questions}

As we have seen above, there have been many experimental results which point to various kinetic schemes for EET and CS in the RC. While the methods above have brought some light to the rates and processes involved, there still remains to be a consensus on the time ordering of the dynamical processes and the role of the different pigments in energy and charge transfer events [29, 32]. Remaining questions include the existence of a subpicosecond energy equilibrium process, the rates for primary charge separation and excitation energy transfer, and the identity of the pigments involved as charge transfer intermediates. Figure 1.5 shows two recent kinetic schemes for charge separation from the literature.

In the $\mathrm{RC}$ and other systems which may exhibit strong coupling between chromophores, an excitation is no longer assumed to be localized to a single chromophore. Instead, we adopt an exciton picture in which the states are delocalized across the involved chromophores and the energy levels are perturbed. To illustrate this, consider a simple dimer consisting of two identical chromophores with strong coupling between them [85]. The Hamiltonian is given by

$$
H=\left(\begin{array}{ll}
E_{1}^{1} & V_{12} \\
V_{21} & E_{2}^{1}
\end{array}\right)
$$

In this simple case, the excited state energy is $E_{1}^{1}=E_{2}^{1}=E$ and coupling $V_{12}=$ $V_{21}=V$. In order to obtain energies for the exciton states, the Hamiltonian must be diagonalized. The eigenenergies will be the energies of the exciton transitions, and the eigenbasis will be the exciton wave functions. To solve for the energies, we take the determinant

$$
\left|\begin{array}{cc}
E-\epsilon & V \\
V & E-\epsilon
\end{array}\right|=0
$$



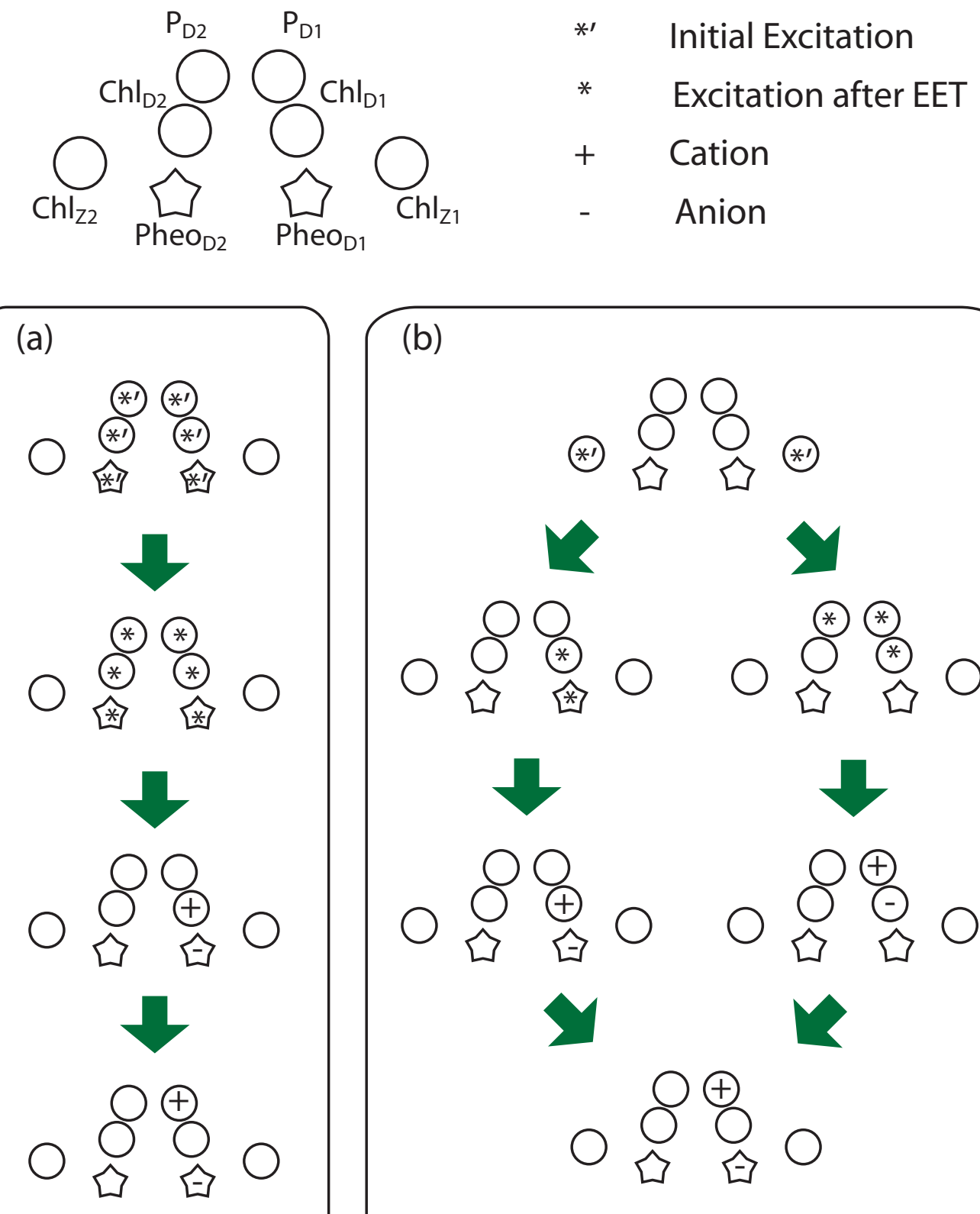

(b)

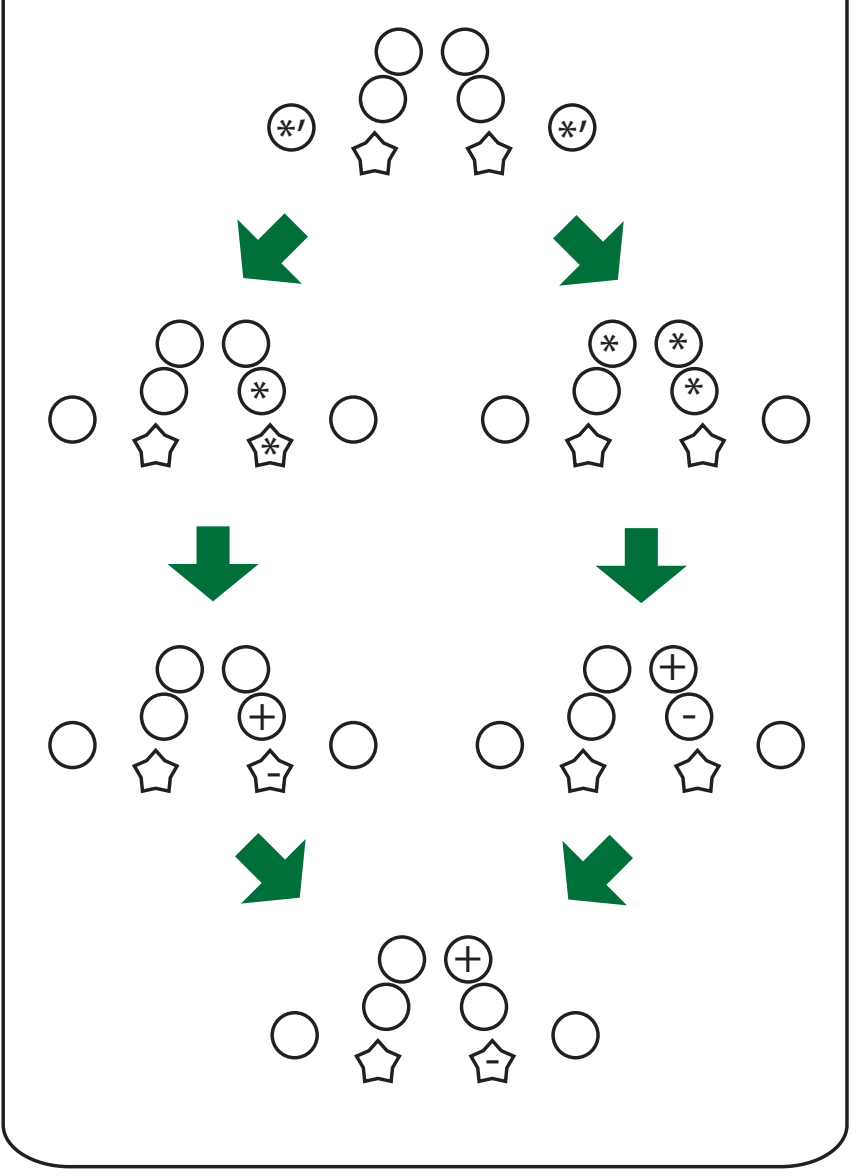

Figure 1.5: Two example kinetic models for the RC from the literature. (a) The model of Prokhorenko et al. [62]. (b) The model of Romero et al. [43], which bears close comparison to kinetics reported from the mutant studies of Diner et al. [84]. The diagrammatic representation has been adapted from [32]. 
This gives

$$
(E-\epsilon)^{2}=V^{2}
$$

Solving for the eigenvalues, we get the exciton energies $\epsilon$ :

$$
\begin{aligned}
& \epsilon_{1}=E+V \\
& \epsilon_{2}=E-V
\end{aligned}
$$

The excitonic coupling therefore splits the energy between the two levels by twice the coupling.

While numerous exciton models have been proposed for the RC [42, 86, 37, 36, 24, 41], these have not been adequately tested by experiment. These competing models differ in their determination of excitonic couplings and the manifold of electronic states. For this thesis, we have chosen the model by Novoderezhkin et al. [86] as a framework in which to present our experimental data. This model is based on quantitative fits of absorption, linear dichroism, steady-state fluorescence, circular dichroism, triplet-minus-singlet, and Stark spectra. It takes into account the spectra of pheophytin-modified RCs and RCs in which one of the peripheral chlorophylls has been removed (RC5). The excited state manifold includes pure exciton states and a charge transfer state, giving a total of 9 states, as shown in Figure 1.6.

\subsection{Two-Dimensional Electronic Spectroscopy}

The difficulty in measuring the spectral dynamics of the $\mathrm{RC}$ with high time and frequency resolution and the inherent problems with resolving dynamics in a highly congested spectral region can be somewhat mitigated by the use of a higher order spectroscopic technique.

One of the newest and most powerful techniques for elucidating spectral dynamics 
Crystal structure of the PS II Reaction Center (3BZ1)

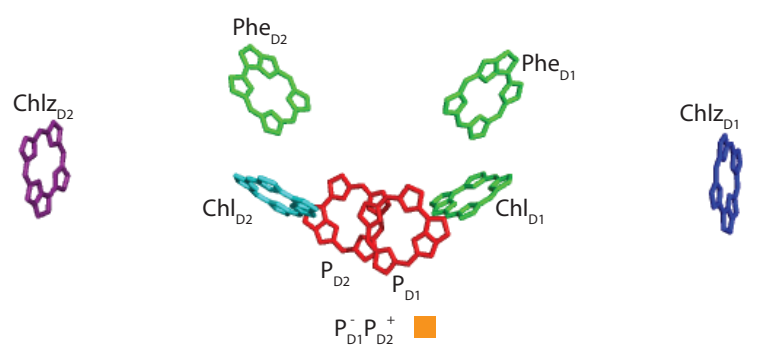

Wavelength of the exciton transition $(\mathrm{nm})$

$\begin{array}{lllllllll}662.0 & 667.0 & 670.0 & 670.8 & 672.1 & 673.4 & 676.8 & 679.5 & 681.0\end{array}$

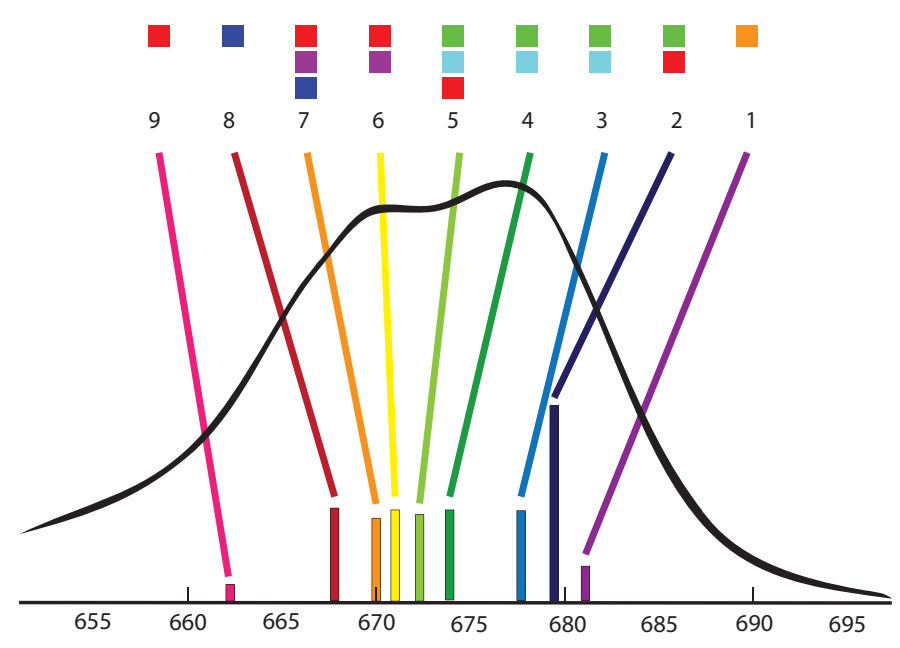

Figure 1.6: Graphical summary of exciton states included in the model of the RC by Novoderezhkin et al. This has been adapted from [86]. The colored squares represent the pigments involved in each exciton state. The states are numbered in order of increasing energy. 
of electronic transitions is two-dimensional electronic spectroscopy (2DES) [87, 88]. Analogous to 2D nuclear magnetic resonance (NMR) spectroscopy, this technique presents spectroscopic data as a map of spectral interactions between two frequencies: $\omega_{1}$ and $\omega_{3}$, which can be thought of as excitation and detection frequencies, respectively. While the remainder of the thesis is devoted to describing the theory and experimental implementations of 2DES and its applications to studying the RC, a brief description is given here to motivate the $\mathrm{RC}$ experiments.

2DES probes the third order polarization generated by the interaction of three carefully timed laser pulses with the sample of interest. First, a broadband pump pulse interacts with the sample, exciting a quantum mechanical coherence between the ground and first excited states. After a time $t_{1}$, frequently called the coherence time, a second pump pulse interacts with the sample, creating a population on the ground or excited electronic state. The time $t_{2}$ before the third pulse arrives is often referred to as the population time, and it is here that many of the interesting dynamics such as energy and charge transfer occur. A probe pulse creates a second coherence in the sample, which leads to signal emission at a time $t_{3}$ after the probe pulse, based on one of two major processes. The first of these, in which the phase progression reverses in comparison to the first coherence, is called the "rephasing" signal or "photon echo". The other follows a free-induction decay along the original phase progression and is called a "non-rephasing" signal or sometimes a "virtual echo". To reconstruct a true absorptive 2D spectrum, both of these signals must be obtained and properly combined [89, 90, 91]. The real part of the complex 2DES spectrum will give the absorptive part and the imaginary part produces a dispersive component. Absorptive spectra are desirable because they feature the narrowest available linewidths, from which the homogeneous and inhomogeneous contributions can be easily measured separately from one another.

To obtain a $2 \mathrm{D}$ spectrum, the population time $\left(t_{2}\right)$ is held constant, but the 
coherence time $\left(t_{1}\right)$ is scanned. Linear transient spectra are obtained in the frequency domain $\left(\omega_{3}\right)$ with a spectrometer for each value of $t_{1}$. A Fourier transform from $t_{1}$ to $\omega_{1}$ effectively represents the coherence time in terms of an excitation frequency, while the measured spectrum in $\omega_{3}$ defines the detection axis. The Fourier transform across $t_{1}$ allows us to benefit from the high time resolution offered with a transform-limited broadband pulse without having to sacrifice frequency selectivity. Heterodyning of the signal pulse with a known local oscillator allows precise retrieval of the signal phase in the $\omega_{3}$ axis, guaranteeing simultaneously high time and frequency resolution along both axes. Knowledge of the signal phase also permits distinction between different physical processes.

To illustrate the types of information available in 2DES data, a cartoon of a simple 2D spectrum is shown in Figure 1.7 along with energy level diagrams indicating the transitions involved in the case of a pair of coupled three-level systems. For cases in which the sample absorbs and emits at the same frequency, a peak shows up along the diagonal, while energy transfer and electronic coupling show up as a cross-peak below the diagonal. Cross-peaks above the diagonal also occur but are generally lower amplitude due to the low probability of uphill energy transfer in most coupling schemes. Excited state absorption is shown as a negative amplitude peak shifted from the diagonal by the anharmonicity. The spectrum also gives a direct measure of the homogeneous and inhomogeneous linewidths as the antidiagonal and diagonal peak widths, which can be used to obtain the frequency-frequency correlation function for a given value of $t_{2}[92]$.

To analyze sample kinetics or any other population dynamics, a series of 2D spectra are acquired, each for a different value of $t_{2}$. One then obtains a complex 3D array of data: $\tilde{S}\left(\omega_{1}, t_{2}, \omega_{3}\right)$. Each $2 \mathrm{D}$ spectrum is a frequency-frequency correlation map: given that a chromophore is excited at a frequency $\omega_{1}$, the $2 \mathrm{D}$ spectrum shows directly the distribution of frequencies $\omega_{3}$ at which the sample emits or absorbs after 

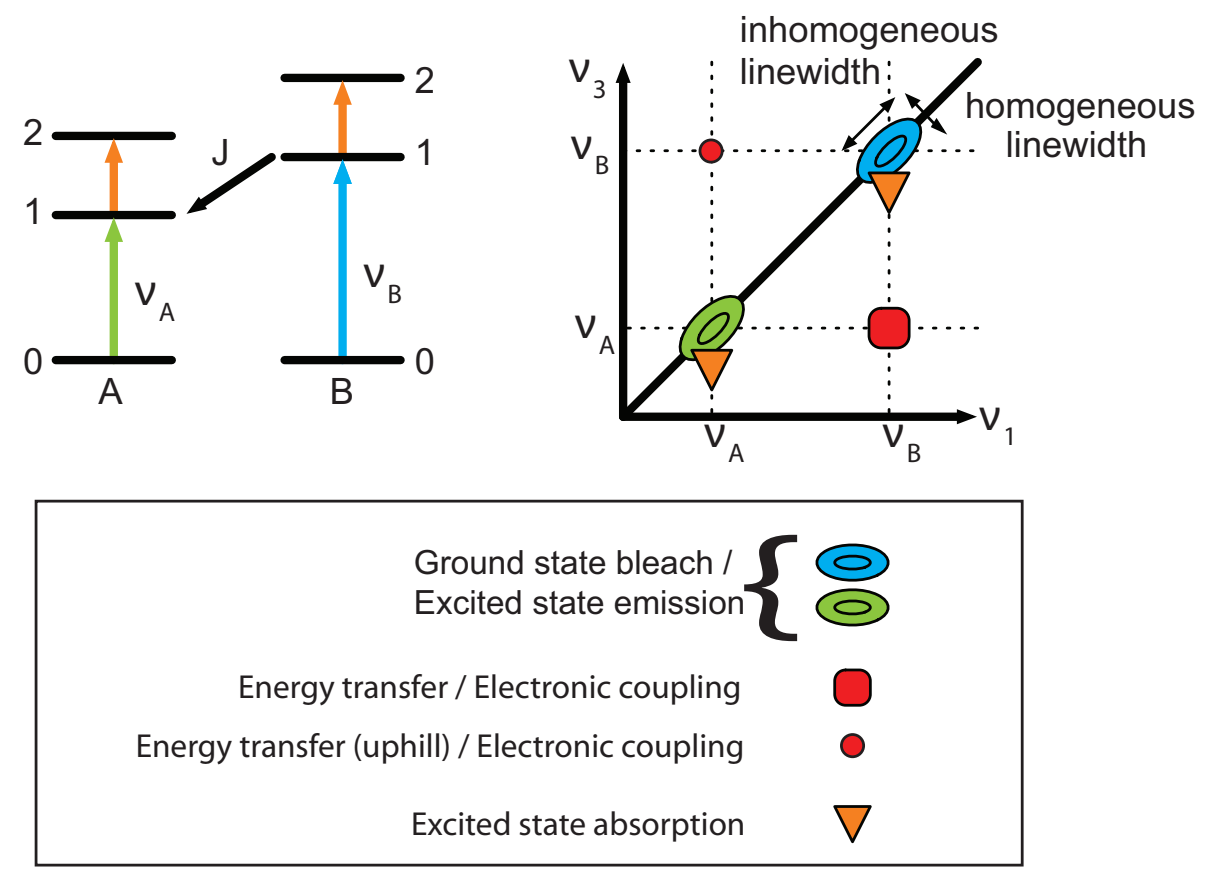

Figure 1.7: A cartoon representation of a 2DES spectrum and associated energy level diagrams for a pair of coupled three-level systems.

a time $t_{2}$.

The information content of a TA experiment is the same as that of a $2 \mathrm{D}$ spectrum integrated over the excitation $\left(\omega_{1}\right)$ axis. The added frequency dimension in 2DES permits separation of different spectroscopic signals that are spectrally overlapped along $\omega_{1}$. Therefore, one intuitive way to think of the $3 \mathrm{D}$ data matrix is as a set of transient absorption spectra taken as a function of $t_{2}$ over a series of narrowband excitation and probe frequencies (a "double resonance" experiment) [87]. In this picture, tracking a single frequency coordinate $\left(\omega_{1}, \omega_{3}\right)$ over $t_{2}$ produces a trace very similar to a narrowband probe TA experiment; alternatively, selecting a single pump frequency $\omega_{1}$, one can obtain the broadband linear transient spectra of a frequencyresolved TA experiment, $\Delta A(\omega, t)$. A key distinction between 2DES and double resonance experiments is that the frequency resolution does not come at the expense of time resolution. With the frequency selectivity and high resolution of 2DES, the 
global fitting and kinetics analysis techniques used in other spectroscopy techniques can be utilized to even greater effect.

2DES is an ideal method for the study of condensed phase systems, including biological complexes in solution, in which the local solvent or protein environment introduces a large degree of inhomogeneous broadening. The technique can reveal homogeneous lineshapes beneath inhomogeneously broadened spectra to provide insight into the physical nature of the broadenin. It can also capture couplings, energy and charge transfer, and other rapid spectral dynamics with high time resolution [88]. The experimental studies utilizing 2DES are still few compared to 2DIR due to the relative difficulty of implementing the technique at visible frequencies, but the technique has been successfully applied to study solvent dynamics [93, 87], energy transfer in semiconductor systems [94, 95, 96, 97], and several photosynthetic systems $[9,98,99,100]$.

\subsection{Scope and content of this work}

This dissertation presents the first 2DES studies of the D1D2-cyt.b559 reaction center from Photosystem II of higher plants. From a biological standpoint, the application of 2DES to study the open questions in such a complex and challenging system is the primary achievement of this work. From a spectroscopist's view, however, this dissertation also presents the implementation of a difficult experimental technique, innovations in the way that the data are obtained, processed, and analyzed, and technical advances aimed at expanding the potential applications of 2DES and simplifying its implementation for other researchers who wish to augment the capabilities of their laboratories to include 2DES. It is my sincere intent to present my body of research with due attention to both of these goals.

Chapter 1.5 will present the theory of 2DES, motivating the technique from the standpoint of available information content and introducing a mathematical frame- 
work with which to understand 2DES. The utility of modelling and its phenomenological interrelationship with experimental 2DES will be briefly presented, though this thesis contains no specific implementations of modelling schemes.

Chapter 2.7 explains the physical apparatus involved in 2DES, the protocol for calibrating the system and obtaining data, and a comparison of two different but complementary implementations of the experiment, both of which have been utilized in our lab. The latter of these implementations, 2DES in the pump-probe geometry with a pulse-shaper, is a major innovation developed in our lab which allows precise control of the $t_{1}$ delay, thus removing timing jitter and providing excellent automatic phase stability for retrieval of clean signals free of distortion. This technique produces automatically phased absorptive spectra which offer a cleaner examination of the data without a need for separate measurements. This chapter also contains an explanation of the data analysis required to produce an absorptive $2 \mathrm{D}$ spectrum, but the specific kinetic analysis of a 3D data matrix will be saved for the following chapter.

Chapter 3.8 begins with a description of the experiments performed on the RC. In the course of analyzing the kinetics of the data, I have developed a new fitting method particular to 2D spectra. The resulting 2D decay associated spectra (2DDAS) are shown along with samples of the raw data, and the results are discussed in the conjunction with other studies on the RC.

Finally, Chapter 4.6 summarizes the studies performed here, enumerates the contributions that the contents of this thesis represent to the field of ultrafast spectroscopy, and discusses future directions of study.

The appendices describe ancillary aspects of the experimental process and preliminary studies, which are no less crucial to the data presented in this thesis than the methods presented in the numbered chapters. They are important for a complete reconstruction of the process. Appendix $\mathrm{V}$ described preliminary experiments on reduced RC samples. Appendix A describes an experimental analysis of our 2D 
data on the $\mathrm{RC}$ in which we examined the spectra for signs of long-lived electronic coherences. Appendix B presents a ZAP-SPIDER device for characterization of our laser pulses, which is one of several such devices one may construct for this task. Appendix $\mathrm{C}$ gives detailed instructions for the purification and spectral characterization of our photosystem II reaction center complexes, written to guide a reader with little knowledge of biochemistry through the practical aspects of purification. Appendix D gives the motivation and design specifications for a custom sample cell for use in a cryostat. 


\section{Bibliography}

[1] Robert E. Blankenship. Molecular Mechanisms of Photosynthesis. Blackwell Science Ltd., 2002.

[2] V. Martinez-Junza, M. Szczepaniak, S. E. Braslavsky, J. Sander, M. Nowaczyk, M. Rogner, and A. R. Holzwarth. A photoprotection mechanism involving the d-2 branch in photosystem ii cores with closed reaction centers. Photochemical $\&$ Photobiological Sciences, 7(11):1337-1343, 2008.

[3] A. Barnett, D. Kirkpatrick, C. Honsberg, D. Moore, M. Wanlass, K. Emery, R. Schwartz, D. Carlson, S. Bowden, D. Aiken, A. Gray, S. Kurtz, L. Kazmerski, M. Steiner, J. Gray, T. Davenport, R. Buelow, L. Takacs, N. Shatz, J. Bortz, O. Jani, K. Goossen, F. Kiamilev, A. Doolittle, I. Ferguson, B. Unger, G. Schmidt, E. Christensen, and D. Salzman. Very high efficiency solar cell modules. Progress In Photovoltaics, 17(1):75-83, January 2009.

[4] J. F. Geisz, D. J. Friedman, J. S. Ward, A. Duda, W. J. Olavarria, T. E. Moriarty, J. T. Kiehl, M. J. Romero, A. G. Norman, and K. M. Jones. $40.8 \%$ efficient inverted triple-junction solar cell with two independently metamorphic junctions. Applied Physics Letters, 93(12), 2008.

[5] D. Gust and T. A. Moore. Mimicking photosynthesis. Science, 244(4900):35-41, 1989.

[6] S. Fukuzumi. Development of bioinspired artificial photosynthetic systems. Physical Chemistry Chemical Physics, 10(17):2283-2297, 2008.

[7] J. Barber. Photosynthetic energy conversion: natural and artificial. Chemical Society Reviews, 38(1):185-196, 2009.

[8] A. V. Pisliakov, T. Mancal, and G. R. Fleming. Two-dimensional optical threepulse photon echo spectroscopy. ii. signatures of coherent electronic motion and exciton population transfer in dimer two-dimensional spectra. Journal of Chemical Physics, 124, 2006.

[9] G. S. Engel, T. R. Calhoun, E. L. Read, T. K. Ahn, T. Mancal, Y. C. Cheng, R. E. Blankenship, and G. R. Fleming. Evidence for wavelike energy transfer through quantum coherence in photosynthetic systems. Nature, 446(7137):782$786,2007$. 
[10] E. Collini and G. D. Scholes. Electronic and vibrational coherences in resonance energy transfer along meh-ppv chains at room temperature. Journal of Physical Chemistry A, 113(16):4223-4241, 2009.

[11] E. Collini, C. Y. Wong, K. E. Wilk, P. M. G. Curmi, P. Brumer, and G. D. Scholes. Coherently wired light-harvesting in photosynthetic marine algae at ambient temperature. Nature, 463(7281):644-U69, 2010.

[12] C. E. Shannon. Communication in the presence of noise. Proceedings of the Institute of Radio Engineers, 37(1):10-21, 1949.

[13] A. H. Zewail. Femtochemistry: Atomic-scale dynamics of the chemical bond using ultrafast lasers - (nobel lecture). Angewandte Chemie-international Edition, 39(15):2587-2631, 2000.

[14] Graham Fleming. Chemical Applications of Ultrafast Spectroscopy. Oxford University Press, 1986.

[15] R. G. W. Norrish and G. Porter. Chemical reactions produced by very high light intensities. Nature, 164(4172):658-658, 1949.

[16] G. Porter. Flash photolysis and spectroscopy a new method for the study of free radical reactions. Proceedings of the Royal Society of London Series Amathematical and Physical Sciences, 200(1061):284-\&, 1950.

[17] F. J. McClung and R. W. Hellwarth. Giant optical pulsations from ruby. Journal of Applied Physics, 33(3):828-\&, 1962.

[18] Amnon Yariv. Quantum Electronics. John Wiley \& Sons, 1975.

[19] D. J. Bradley and G. H. C. New. Ultrashort pulse measurements. Proceedings of the Ieee, 62(3):313-345, 1974.

[20] M. J. Rosker, M. Dantus, and A. H. Zewail. Femtosecond clocking of the chemical-bond. Science, 241(4870):1200-1202, 1988.

[21] M. Drescher, M. Hentschel, R. Kienberger, G. Tempea, C. Spielmann, G. A. Reider, P. B. Corkum, and F. Krausz. X-ray pulses approaching the attosecond frontier. Science, 291(5510):1923-1927, March 2001.

[22] M. Hentschel, R. Kienberger, C. Spielmann, G. A. Reider, N. Milosevic, T. Brabec, P. Corkum, U. Heinzmann, M. Drescher, and F. Krausz. Attosecond metrology. Nature, 414(6863):509-513, November 2001.

[23] J. Itatani, J. Levesque, D. Zeidler, H. Niikura, H. Pepin, J. C. Kieffer, P. B. Corkum, and D. M. Villeneuve. Tomographic imaging of molecular orbitals. Nature, 432(7019):867-871, December 2004.

[24] N. Ivashin and S. Larsson. Excitonic states in photosystem ii reaction center. Journal of Physical Chemistry B, 109(48):23051-23060, 2005. 
[25] G. Renger and T. Renger. Photosystem ii: The machinery of photosynthetic water splitting. Photosynthesis Research, 98(1-3):53-80, 2008.

[26] Donald Voet and Judith G Voet. Biochemistry. John Wiley \& Sons, 3 edition, 2004.

[27] M Kobayashi, H Maeda, T Watanabe, H Nakane, and K Satoh. Chlorophyllalpha and beta-carotene content in the $\mathrm{d} 1 / \mathrm{d} 2 /$ cytochrome-b-559 reaction center complex from spinach. Febs Letters, 260(1):138-140, JAN 151990.

[28] C Eijkelhoff and JP Dekker. Determination of the pigment stoichiometry of the photochemical-reaction center of photosystem-ii. Biochemica et Biophysica Acta-Bioenergetics, 1231(1):21-28, AUG 151995.

[29] SR Greenfield and MR Wasielewski. Excitation energy transfer and charge separation in the isolated photosystem ii reaction center. Photosynthesis Research, 48(1-2):83-97, MAY 1996.

[30] M. G.; Reus M.; Nowaczyk M.; Sander J. \& Rogner M. Holzwarth, A. R.; Muller. Kinetics and mechanism of electron transfer in intact photosystem ii and in the isolated reaction center: Pheophytin is the primary electron acceptor. Proceedings of the National Academy of Sciences of the United States of America, 103(18):6895-6900, 2006.

[31] J. Hasegawa, Y. Ozeki, K. Ohkawa, M. Hada, and H. Nakatsuji. Theoretical study of the excited states of chlorin, bacteriochlorin, pheophytin a and chlorophyll a by the sac/sac-ci method. Journal of Physical Chemistry B, 102(7):13201326, February 1998.

[32] LM Yoder, AG Cole, and RJ Sension. Structure and function in the isolated reaction center complex of photosystem ii: energy and charge transfer dynamics and mechanism. Photosynthesis Research, 72(2):147-158, 2002.

[33] R Jankowiak, D Tang, GJ Small, and M Seibert. Transient and persistent hole burning of the reaction center of photosystem-ii. Journal of Physical Chemistry, 93(4):1649-1654, FEB 231989.

[34] V. L. Tetenkin, B. A. Gulyaev, M. Seibert, and A. B. Rubin. Spectral properties of stabilized d1/d2/cytochrome-b-559 photosystem-ii reaction center complex effects of triton x-100, the redox state of pheophytin, and beta-carotene. Febs Letters, 250(2):459-463, July 1989.

[35] C Eijckelhoff, $\mathrm{H}$ vanRoon, ML Groot, $\mathrm{R}$ vanGrondelle, and JP Dekker. Purification and spectroscopic characterization of photosystem ii reaction center complexes isolated with or without triton x-100. Biochemistry, 35(39):1286412872, OCT 11996. 
[36] B. Svensson, C. Etchebest, P. Tuffery, P. vanKan, J. Smith, and S. Styring. A model for the photosystem ii reaction center core including the structure of the primary donor p-680. Biochemistry, 35(46):14486-14502, November 1996.

[37] J. R. Durrant, D. R. Klug, S. L. S. Kwa, R. van Grondelle, G. Porter, and J. P. Dekker. A multimer model for p680, the primary electron-donor of photosystemii. Proceedings of the National Academy of Sciences of the United States of America, 92(11):4798-4802, May 1995.

[38] N. Kamiya and J. R. Shen. Crystal structure of oxygen-evolving photosystem ii from thermosynechococcus vulcanus at 3.7-angstrom resolution. Proceedings of the National Academy of Sciences of the United States of America, 100(1):98103, 2003.

[39] A. Guskov, J. Kern, A. Gabdulkhakov, M. Broser, A. Zouni, and W. Saenger. Cyanobacterial photosystem ii at 2.9-angstrom resolution and the role of quinones, lipids, channels and chloride. Nature Structural \& Molecular Biology, 16(3):334-342, 2009.

[40] S. R. Greenfield, M. Seibert, and M. R. Wasielewski. Time-resolved absorption changes of the pheophytin $\mathrm{q}(\mathrm{x})$ band in isolated photosystem ii reaction centers at $7 \mathrm{k}$ : Energy transfer and charge separation. Journal of Physical Chemistry B, 103(39):8364-8374, September 1999.

[41] L. M. C. Barter, J. R. Durrant, and D. R. Klug. A quantitative structurefunction relationship for the photosystern ii reaction center: Supermolecular behavior in natural photosynthesis. Proceedings of the National Academy of Sciences of the United States of America, 100(3):946-951, 2003.

[42] G. Raszewski, B. A. Diner, E. Schlodder, and T. Renger. Spectroscopic properties of reaction center pigments in photosystem ii core complexes: Revision of the multimer model. Biophysical Journal, 95(1):105-119, 2008.

[43] E. Romero, I. H. M. van Stokkum, V. I. Novoderezhkin, J. P. Dekker, and R. van Grondelle. Two different charge separation pathways in photosystem ii. Biochemistry, 49(20):4300-4307, 2010.

[44] O. Nanba and K. Satoh. Isolation of a photosystem-ii reaction center consisting of d-1 and d-2 polypeptides and cytochrome-b-559. Proceedings of the National Academy of Sciences of the United States of America, 84(1):109-112, 1987.

[45] TA Roelofs, M Gilbert, VA Shuvalov, and AR Holzwarth. Picosecond Fluorescence Kinetics of the D1-D2-Cyt-B-559 Photosystem-II Reaction Center Complex - Energy-Transfer and Primary Charge Separation Processes. Biochemica et Biophysica Acta, 1060(3):237-244, NOV 71991.

[46] TA Roelofs, SLS Kwa, R van Grondelle, JP Dekker, and AR Holzwarth. Primary Processes and Structure of the Photosystem II Reaction-Center .2. 
Low-Tempterature Picosecond Fluorescence Kinetics of a D1-D2-Cyt-B-559 Reaction-Center Complex Isolated by Short Triton Exposure. Biochemica et Biophysica Acta, 1143(2):147-157, JUL 51993.

[47] AR Holzwarth, MG Muller, G Gatzen, M Hucke, and K Griebenow. Ultrafast Spectroscopy of the Primary Electron and Energy-Transfer Processes in the Reaction-Center of Photosystem-II. Journal of Luminescence, 60-1:497-502, APR 1994. 1993 International Conference on Luminescence (ICL 93), STORRS, CT, AUG 09-13, 1993.

[48] A Freiberg, K Timpmann, AA Moskalenko, and NY Kuznetsova. Picosecond and Nanosecond Fluorescence Kinetics of Photosystem-II Reaction-Center and its Complex With CP47 Antenna. Biochemica et Biophysica Acta-Bioenergetics, 1184(1):45-53, FEB 81994.

[49] G. Gatzen, M. G. Muller, K. Griebenow, and A. R. Holzwarth. Primary processes and structure of the photosystem ii reaction center .3. kinetic analysis of picosecond energy transfer and charge separation processes in the d1-d2cyt-b559 complex measured by time-resolved fluorescence. Journal of Physical Chemistry, 100(17):7269-7278, April 1996.

[50] B Donovan, LA Walker, D Kaplan, M Bouvier, CF Yocum, and RJ Sension. Structure and function in the isolated reaction center complex of photosystem ii .1. ultrafast fluorescence measurements of psii. Journal of Physical Chemistry B, 101(26):5232-5238, JUN 261997.

[51] L. Konermann, I. Yruela, and A. R. Holzwarth. Pigment assignment in the absorption spectrum of the photosystem ii reaction center by site-selection fluorescence spectroscopy. Biochemistry, 36(24):7498-7502, June 1997.

[52] M. L.; van Grondelle R.; Dekker J. P. \& van Stokkum I. H. M. van Mourik, F.; Groot. Global and target analysis of fluorescence measurements on photosystem 2 reaction centers upon red excitation. Physical Chemistry Chemical Physics, 6(20):4820-4824, 2004.

[53] S. Kumazaki, D. M. Joseph, B. Crystall, Y. Tachibana, J. R. Durrant, J. Barber, G. Porter, K. Yoshihara, and D. R. Klug. Experimental observation of multiple trapping charge separation steps in the isolated ps2 reaction centre. Photosynthesis: From Light To Biosphere, Vol I, pages 883-886, 1995.

[54] D. Tang, R. Jankowiak, M. Seibert, C. F. Yocum, and G. J. Small. Excitedstate structure and energy-transfer dynamics of 2 different preparations of the reaction center of photosystem-ii - a hole-burning study. Journal of Physical Chemistry, 94(17):6519-6522, August 1990.

[55] D. M. Tang, R. Jankowiak, M. Seibert, and G. J. Small. Effects of detergent on the excited-state structure and relaxation dynamics of the photosystem-ii 
reaction center - a high-resolution hole burning study. Photosynthesis Research, 27(1):19-29, 1991.

[56] R. Jankowiak, M. Ratsep, R. Picorel, M. Seibert, and G. J. Small. Excited states of the 5-chlorophyll photosystem ii reaction center. Journal of Physical Chemistry B, 103(44):9759-9769, November 1999.

[57] V. Zazubovich, R. Jankowiak, K. Riley, R. Picorel, M. Seibert, and G. J. Small. How fast is excitation energy transfer in the photosystem ii reaction center in the low temperature limit? hole burning vs photon echo. Journal of Physical Chemistry B, 107(12):2862-2866, 2003.

[58] M.; Hayes J.; Zazubovich V.; Picorel R.; Seibert M. \& Small G. J. Jankowiak, R.; Ratsep. Primary charge-separation rate at $5 \mathrm{k}$ in isolated photosystem ii reaction centers containing five and six chlorophyll a molecules. Journal of Physical Chemistry B, 107(9):2068-2074, 2003.

[59] K. Riley, R. Jankowiak, M. Ratsep, G. J. Small, and V. Zazubovich. Evidence for highly dispersive primary charge separation kinetics and gross heterogeneity in the isolated psii reaction center of green plants. Journal of Physical Chemistry B, 108(29):10346-10356, July 2004.

[60] K. J. Vink, S. Deboer, J. J. Plijter, A. J. Hoff, and D. A. Wiersma. Opticaldynamics of the reaction center of photosystem-ii - a hole-burning and photonecho study. Chemical Physics Letters, 142(6):433-438, 1987.

[61] R. J. W. Louwe and T. J. Aartsma. Excited state dynamics in photosynthetic antenna complexes studied with accumulated photon echoes. Photosynthesis: From Light To Biosphere, Vol I, pages 363-366, 1995.

[62] V. I. Prokhorenko and A. R. Holzwarth. Primary processes and structure of the photosystem ii reaction center: A photon echo study. Journal of Physical Chemistry B, 104(48):11563-11578, 2000.

[63] MR Wasielewski, DG Johnson, M Seibert, and Govindjee. Determination of the Primary Charge Separation Rate in Isolated Photosystem-II Reaction Centers with 500-fs Time Resolution. Proceedings of the National Academy of Sciences of the United States of America, 86(2):524-528, JAN 1989.

[64] MR Wasielewski, DG Johnson, Govindjee, C Preston, and M Seibert. Determination of the Primary Charge Separation Rate in Photosystem-II Reaction Centers at 15-K. Photosynthesis Research, 22(1):89-99, OCT 1989.

[65] JR Durrant, G Hastings, DM Joseph, J Barber, G Porter, and DR Klug. Subpicosecond Equilibration of Excitation-Energy in Isolated Photosystem-II Reaction Centers. Proceedings of the National Academy of Sciences of the United States of America, 89(23):11632-11636, DEC 11992. 
[66] J. R. Durrant, G. Hastings, Q. A. Hong, J. Barber, G. Porter, and D. R. Klug. Determination of p680 singlet-state lifetimes in photosystem 2 reaction centers. Chemical Physics Letters, 188(1-2):54-60, 1992.

[67] G Hastings, JR Durrant, J Barber, G Porter, and DR Klug. Observation of Pheophytin Reduction in Photosystem 2 Reaction Centers Using Femtosecond Transient Absorption-Spectroscopy. Biochemistry, 31(33):7638-7647, AUG 25 1992.

[68] S. W. McCauley, A. P. Baronavski, J. K. Rice, M. L. GhirardiI, and A. K. Mattoo. A search for subpicosecond absorption components in photosystem-ii reaction centers. Chemical Physics Letters, 198(5):437-442, 1992.

[69] JR Durrant, G Hastings, DM Joseph, J Barber, G Porter, and DR Klug. Rate of Oxidation of P680 in Isolated Photosystem-2 Reaction Centers Monitored by Loss of Chlorophyll Stimulated-Emission. Biochemistry, 32(32):8259-8267, AUG 171993.

[70] T Rech, JR Durrant, DM Joseph, J Barber, G Porter, and DR Klug. Does Slow Energy-Transfer Limit the Observed Time Constant for Radical Pair Formation in Photosystem-II Reaction Centers. Biochemistry, 33(49):14768-14774, DEC 131994.

[71] JPM Schelvis, PI van Nort, TJ Aartsma, and HJ van Gorkom. EnergyTransfer, Charge Separation and Pigment Arrangement in the Reaction-Center of Photosystem-II. Biochemica et Biophysica Acta-Bioenergetics, 1184(23):242-250, MAR 81994.

[72] F. Vacha, D. M. Joseph, J. R. Durrant, A. Telfer, D. R. Klug, G. Porter, and J. Barber. Photochemistry and spectroscopy of a 5-chlorophyll reaction-center of photosystem-ii isolated by using a cu affinity column. Proceedings of the National Academy of Sciences of the United States of America, 92(7):29292933, 1995.

[73] G. P. Wiederrecht, M. Seibert, Govindjee, and M. R. Wasielewski. Femtosecond photodichroism studies of isolated photosystem-ii reaction centers. Proceedings of the National Academy of Sciences of the United States of America, 91(19):8999-9003, 1994.

[74] DR Klug, T Rech, DM Joseph, J Barber, JR Durrant, and G Porter. Primary Processes in Isolated Photosystem-II Reaction Centers Probed by Magic-Angle Transient Absorption-Spectroscopy. Chemical Physics, 194(2-3):433-442, MAY 151995.

[75] B Donovan, LA Walker, CF Yocum, and RJ Sension. Transient absorption studies of the primary charge separation in photosystem II. Journal of Physical Chemistry, 100(5):1945-1949, FEB 11996. 
[76] M. G. Muller, M. Hucke, M. Reus, and A. R. Holzwarth. Primary processes and structure of the photosystem ii reaction center .4. low-intensity femtosecond transient absorption spectra of d1-d2-cyt-b559 reaction center. Journal of Physical Chemistry, 100(22):9527-9536, May 1996.

[77] S. R. Greenfield, M. Seibert, Govindjee, and M. R. Wasielewski. Direct measurement of the effective rate constant for primary charge separation in isolated photosystem ii reaction centers. Journal of Physical Chemistry B, 101(13):22512255, March 1997.

[78] S. R. Greenfield, M. Seibert, Govindjee, and M. R. Wasielewski. Wavelength and intensity dependent primary photochemistry of isolated photosystem ii reaction centers at 5 degrees c. Chemical Physics, 210(1-2):279-295, 1996.

[79] HM VIisser, ML Groot, F van Mourik, IHM van Stokkum, JP Dekker, and $\mathrm{R}$ van Grondelle. Subpicosecond Transient Absorption Difference Spectroscopy on the Reaction-Center of Photosystem-II - Radical Pair Formation at 77-K. Journal of Physical Chemistry, 99(41):15304-15309, OCT 121995.

[80] S. A. P. Merry, S. Kumazaki, Y. Tachibana, D. M. Joseph, G. Porter, K. Yoshihara, J. Barber, J. R. Durrant, and D. R. Klug. Sub-picosecond equilibration of excitation energy in isolated photosystem ii reaction centers revisited: Timedependent anisotropy. Journal of Physical Chemistry, 100(24):10469-10478, June 1996.

[81] M. L. Groot, F. vanMourik, C. Eijckelhoff, I. H. M. vanStokkum, J. P. Dekker, and $\mathrm{R}$. vanGrondelle. Charge separation in the reaction center of photosystem ii studied as a function of temperature. Proceedings of the National Academy of Sciences of the United States of America, 94(9):4389-4394, April 1997.

[82] M. L. Groot, N. P. Pawlowicz, L. J. G. W. van Wilderen, J. Breton, I. H. M. van Stokkum, and R. van Grondelle. Initial electron donor and acceptor in isolated photosystem ii reaction centers identified with femtosecond mid-ir spectroscopy. Proceedings of the National Academy of Sciences of the United States of America, 102(37):13087-13092, 2005.

[83] M. L.; van Stokkum I. H. M.; Breton J. \& van Grondelle R. Pawlowicz, N. P.; Groot. Charge separation and energy transfer in the photosystem ii core complex studied by femtosecond midinfrared spectroscopy. Biophysical Journal, 93(8):2732-2742, 2007.

[84] B. A. Diner, E. Schlodder, P. J. Nixon, W. J. Coleman, F. Rappaport, J. Lavergne, W. F. J. Vermaas, and D. A. Chisholm. Site-directed mutations at d1-his198 and d2-his 97 of photosystem ii in synechocystis pcc 6803: Sites of primary charge separation and cation and triplet stabilization. Biochemistry, 40(31):9265-9281, 2001. 
[85] Herbert van Amerongen, Leonas Valkunas, and Rienk van Grondelle. Photosynthetic Excitons. World Scientific, 2000.

[86] V. I. Novoderezhkin, J. P. Dekker, and R. van Grondelle. Mixing of exciton and charge-transfer states in photosystem ii reaction centers: Modeling of stark spectra with modified redfield theory. Biophysical Journal, 93:1293-1311, 2007.

[87] D. M. Jonas. Two-dimensional femtosecond spectroscopy. Annual Review of Physical Chemistry, 54:425-463, 2003.

[88] J. P. Ogilvie and K. J. Kubarych. Multidimensional electronic and vibrational spectroscopy: An ultrafast probe of molecular relaxation and reaction dynamics. Advances In Atomic, Molecular, and Optical Physics, Vol 57, 57:249-321, 2009.

[89] SMG Faeder and DM Jonas. Two-dimensional electronic correlation and relaxation spectra: Theory and model calculations. Journal of Physical Chemistry A, 103(49):10489-10505, DEC 91999.

[90] JD Hybl, AA Ferro, and DM Jonas. Two-dimensional Fourier transform electronic spectroscopy. Journal of Chemical Physics, 115(14):6606-6622, OCT 8 2001.

[91] M. Khalil, N. Demirdoven, and A. Tokmakoff. Obtaining absorptive line shapes in two-dimensional infrared vibrational correlation spectra. Physical Review Letters, 90(4), 2003.

[92] Kees Lazonder, Maxim S. Pshenichnikov, and Douwe A. Wiersma. Easy interpretation of optical two-dimensional correlation spectra. Optics Letters, 31(22):3354-3356, NOV 152006.

[93] JD Hybl, A Yu, DA Farrow, and DM Jonas. Polar solvation dynamics in the femtosecond evolution of two-dimensional Fourier transform spectra. Journal of Physical Chemistry A, 106(34):7651-7654, AUG 292002.

[94] C. N. Borca, T. H. Zhang, X. Q. Li, and S. T. Cundiff. Optical two-dimensional fourier transform spectroscopy of semiconductors. Chemical Physics Letters, 416(4-6):311-315, 2005.

[95] XQ Li, TH Zhang, CN Borca, and ST Cundiff. Many-body interactions in semiconductors probed by optical two-dimensional Fourier transform spectroscopy. Physical Review Letters, 96(5), FEB 102006.

[96] Lijun Yang, Igor V. Schweigert, Steven T. Cundiff, and Shaul Mukamel. Twodimensional optical spectroscopy of excitons in semiconductor quantum wells: Liouville-space pathway analysis. Physical Review B, 75(12), MAR 2007.

[97] Tianhao Zhang, Irina Kuznetsova, Torsten Meier, Xiaoclin Li, Richard P. Mirin, Peter Thomas, and Steven T. Cundiff. Polarization-dependent optical 2D Fourier transform spectroscopy of semiconductors. Proceedings of the National 
Academy of Sciences of the United States of America, 104(36):14227-14232, SEP 42007.

[98] T Brixner, J Stenger, HM Vaswani, M Cho, RE Blankenship, and GR Fleming. Two-dimensional spectroscopy of electronic couplings in photosynthesis. Nature, 434(7033):625-628, MAR 312005.

[99] Donatas Zigmantas, Elizabeth L. Read, Tomas Mancal, Tobias Brixner, Alastair T. Gardiner, Richard J. Cogdell, and Graham R. Fleming. Two-dimensional electronic spectroscopy of the B800-B820 light-harvesting complex. Proceedings of the National Academy of Sciences of the United States of America, 103(34):12672-12677, AUG 222006.

[100] Elizabeth L. Read, Gabriela S. Schlau-Cohen, Gregory S. Engel, Jianzhong Wen, Robert E. Blankenship, and Graham R. Fleming. Visualization of excitonic structure in the Fenna-Matthews-Olson photosynthetic complex by polarization-dependent two-dimensional electronic spectroscopy. Biophysical Journal, 95(2):847-856, JUL 152008. 


\section{CHAPTER II}

\section{Theory of 2DES}

In this chapter, I present a theoretical background regarding the formalism of $2 \mathrm{D}$ electronic spectroscopy. Beginning with a discussion of third-order polarization and phase matching, I will present a summary of coherent nonlinear signals available in four-wave mixing experiments and a description of what they can tell us about the system in question. I will further define the different contributions to the signal in terms of double-sided Feynman diagrams and explain how these are read and how to understand a $2 \mathrm{D}$ spectrum in terms of these excitation pathways.

While the experimental methods, analysis techniques, and visual representation and interpretation of spectra bear much similarity to the already well-developed field of multidimensional NMR spectroscopy, there are several unique aspects of electronic and vibrational multidimensional spectroscopy that demand a different experimental treatment. The parallels and differences with respect to 2D NMR will be highlighted throughout this section.

While I will present a mathematical framework for understanding the effects of radiation fields on the system polarization and resulting signals, it is not my intention here to derive a complete theoretical architecture for nonlinear spectroscopy - that is beyond the scope of this work. A brief overview of several prevailing models will be given, but none of these will be presented in detail. This chapter is meant to 
act as an introduction to the theory behind 2DES and to offer a motivation for the technique and a roadmap for the interpretation of the $2 \mathrm{D}$ spectra. For those who desire a rigorous mathematical treatment, Shaul Mukamel's Principles of Nonlinear Optical Spectroscopy and Minhaeng Cho's Two-Dimensional Optical Spectroscopy are excellent reference books $[1,2]$.

\subsection{Motivation: The Limitations of Linear Methods}

Linear spectroscopy measures the first-order polarization induced in a sample by a single interaction with the incident electric field. The most basic method of linear absorption spectroscopy gives a picture of the first excited state manifold of the sample, projected onto a single frequency axis. Transition dipole strengths and excitation energies can be probed, but there is no explicit information about exciton coupling or excited state absorption.

Traditionally, the spectral width of an electronic resonance is separated into homogeneous and inhomogeneous contributions. The homogeneous width is generally a Lorentzian function related directly to an excited state lifetime by an uncertainty condition. In atomic and molecular optics, the traditional example of inhomogeneity is Doppler broadening, in which a distribution of particle velocities in the gas or plasma phase will shift the emission frequency of some particles relative to one another [3].

In the condensed phase, this situation is rather different: chromophores are constantly interacting with the surrounding solvent bath, which leads to fluctuations of the eigenstate energies on timescales similar to the dephasing time. For aggregates or biological molecules, the structural arrangement of molecules (e.g., in a protein matrix) can cause sizeable shifts of the transition frequencies of otherwise identical chromophores at different sites. This disorder leads to a static inhomogeneity which can be further affected by dynamics such as conformational changes or bath reorganization. Condensed phase chromophores can interact with each other as well 
through dipole-dipole interactions. Strong coupling between closely-spaced molecules causes energy levels of individual absorbers to effectively split into delocalized excited states shared across multiple chromophores, or excitons. Linear spectra only probe the inhomogeneous lineshapes involved in transitions between the ground and first excited states and are unable to provide information about dynamics underlying the lineshapes, electronic coupling, or site disorder. For molecular complexes, such as photosynthetic antennas and reaction centers, the linear spectra can become congested and extremely difficult to interpret, even in terms of extracting the transition dipoles or excitation energies of individual states. A higher-order spectroscopic investigation of a condensed phase system can reveal information about the solvent environment, system-bath coupling, and molecular disorder to augment the information available in linear spectra [1].

Methods of nonlinear spectroscopy allow researchers to probe the dynamics and coupling between exciton states by allowing multiple field-matter interactions so that higher orders of the polarization can be measured. An $N+1$-wave mixing experiment is one that involves $N$ field-matter interactions between the sample and external input pulses and one signal pulse, which reveals information pertaining to the material response. Nonlinear techniques can be coherent or incoherent: in coherent experiments, the phase of the pulses is an important factor. This is not the case in incoherent techniques, which are phase-independent [4]. Most of the nonlinear techniques of interest in isotropic systems are four-wave mixing (FWM) techniques.

It was a common mode of thought in the past that all FWM experiments should be able to access the same variety of information, based merely on the number of pulse interactions. More recent thinking classifies spectroscopy techniques by the number of controllable coherence time delays rather than on the number of fieldmatter interactions, as a limiting factor on available molecular information [5]. In 2D spectroscopy, two coherence time delays $\left(t_{1}\right.$ and $\left.t_{3}\right)$ are controlled, with precise phase 
stability, to produce a single $2 \mathrm{D}$ spectrum. The third time delay $\left(t_{2}\right)$ is also tunable in our experiment, and measuring spectra for different $t_{2}$ values provides information on population kinetics and any coherences present during the population time [6]. As we shall present below, the data is given a more intuitive interpretation when displayed in the frequency-frequency domain.

\subsection{Polarization and the density matrix}

The theory applied to 2D spectroscopy is generally a mixed quantum-classical framework, in which the incident fields are treated classically, but the material system is described quantum mechanically. The treatment of the bath and the system-bath coupling usually involves averaging over bath degrees of freedom that are irrelevant to the final spectrum but can vary depending on the modelling framework. The system is described by a density matrix $\rho$. The diagonal elements $\rho_{i i}$ represent populations, while the off-diagonal elements $\rho_{i j}(i \neq j)$ give the coherences.

In NMR, it is typical to represent the density matrix in terms of a three-dimensional Bloch vector $s(t)$. The field-matter interaction is expressed in terms of torques applied by interactions with the field [3]. In this case, the density matrix is encoded in the components of $s(t)$. The equations of motion can then be solved through integration. This treatment is very useful in the strong-field case of NMR. The manifold of states in that system is a set of two-level spins. There are few parameters, and the structures can best be resolved if saturating pulses are used to completely invert the population in the sample volume. This allows for a long series of phase-cycled pulses to access high-order polarization terms and select specific signal contributions without loss of signal strength.

Unfortunately, this kind of approach does not transfer well to the electronic and IR regimes. The manifold of states generally consists of several levels per chromophore, including both electronic multiply-excited states and vibrational substates. Unlike the 
nuclear case, the dipole strength and orientation varies within samples, thus adding a great deal of parameters to the calculations. High intensity pulses can have more than one interaction with the sample chromophores, thus accessing higher order signals than are desired in the experiment. These signals may be spatially and spectrally overlapped with the desired signals, complicating data analysis and obscuring the desired features in a fashion that may not be predictable or correctable in postprocessing. In certain systems, including the D1D2-cyt.b559 reaction center studied in this thesis, multiple excitations of a single complex can lead to exciton annihilation, which distorts the kinetics and reduces the yield of charge separation events [7, 8]. Saturating fields can also lead to reductions in accessible sample molecules over the course of an experiment due to funnelling of energy into long-lived triplet states, photobleaching, and optically induced damage. This is of express concern in biological samples, which are far less robust than dye molecules.

Because of this, 2DES and 2DIR are performed in the weak-field limit, in which only a small percentage of sample molecules are excited and contribute to the signal. In Bloch space, this corresponds to a small angle between the Bloch vector and the ground state population axis rather than a complete inversion. A point-to-point comparison between 2D NMR and 2DES is given in Table 2.1.

In situations of weak excitation, the time dependence of the density matrix may be expanded perturbatively in powers of the incident electric field:

$$
\rho(t) \equiv \rho^{(0)}(t)+\rho^{(1)}(t)+\rho^{(2)}(t)+\ldots
$$

where $\rho^{(n)}(t)$ represents the $\mathrm{n}^{\text {th }}$ order contribution of the electric field. The $\mathrm{n}^{\text {th }}$ order density matrix cannot be measured directly. However, it can be probed by measuring the $\mathrm{n}^{\text {th }}$ order macroscopic polarization, which is related to the expectation value of 


\begin{tabular}{|c|c|c|}
\hline & 2D NMR & 2DES \\
\hline Frequency & $\mathrm{MHz}$ & $10^{14}-10^{15} \mathrm{~Hz}$ \\
\hline Time Resolution & Millisecond & Femtosecond \\
\hline Pulse Intensity & $\begin{array}{l}\text { Strong saturating pulses } \\
\text { All spins excited } \\
\text { Many-pulse sequences possible }\end{array}$ & $\begin{array}{l}\text { Weak pulses } \\
\text { Only a few molecules excited } \\
\text { Only a few pulses per sequence }\end{array}$ \\
\hline $\begin{array}{l}\text { Directionality } \\
\text { of Signal }\end{array}$ & $\begin{array}{l}\lambda \gg \text { sample size } \\
k r \ll 1 \\
\text { Signal is isotropic in space }\end{array}$ & $\begin{array}{l}\lambda \ll \text { sample size } \\
k r \gg 1 \\
\text { Highly directional signal }\end{array}$ \\
\hline $\begin{array}{l}\text { Pathway } \\
\text { selection }\end{array}$ & Phase cycling & Phase matching \\
\hline $\begin{array}{l}\text { Modelling and } \\
\text { calculation }\end{array}$ & $\begin{array}{l}\text { Few parameters } \\
\text { Equal and aligned dipoles } \\
\text { Bloch picture treatment }\end{array}$ & $\begin{array}{l}\text { Many parameters } \\
\text { Dipole strength and orientation } \\
\text { varies } \\
\text { Response function and } \\
\text { density matrix formalism }\end{array}$ \\
\hline Data Analysis & $\begin{array}{l}\text { Spectra can be directly inverted } \\
\text { into structural data }\end{array}$ & $\begin{array}{l}\text { Complete interpretation requires } \\
\text { phenomenological comparison } \\
\text { with models }\end{array}$ \\
\hline
\end{tabular}

Table 2.1: A comparison between 2D NMR and 2DES methods. This has been adapted from [4] 
the dipole operator $(\mu)$ and chromophore number density $(N)$ :

$$
\mathbf{P}^{(n)}(\mathbf{r}, t)=N \operatorname{Tr}\left[\mu \rho^{(n)}(\mathbf{r}, t)\right]
$$

The first order polarization is responsible for linear optical effects. The second order polarization $\left(P^{(2)}\right)$ is involved with nonlinear properties in birefringent media, such as sum and difference frequency generation. It can be shown that the signal contribution from $P^{(2)}$ vanishes in isotropic media [1]. Therefore, the next term of interest after the linear optics is $P^{(3)}$, the third order polarization. It is this order that is probed in 2DES and other four-wave mixing spectroscopies, such as transient absorption. From this point on, I will cease to generalize to the $\mathrm{n}^{\text {th }}$ order and discuss $P^{(3)}$ explicitly, as this is the quantity of interest in the experiments discussed throughout the rest of this thesis.

In the time domain, the third order polarization is a convolution of the incident electric fields with the third order material response function $R^{(3)}\left(t_{3}, t_{2}, t_{1}\right)$ [1]:

$$
\begin{aligned}
\mathbf{P}^{(3)}(\mathbf{r}, t)= & N \int_{0}^{\infty} d t_{3} \int_{0}^{\infty} d t_{2} \int_{0}^{\infty} d t_{1} R^{(3)}\left(t_{3}, t_{2}, t_{1}\right) \\
& \otimes E_{3}\left(\mathbf{r}, t-t_{3}\right) E_{2}\left(\mathbf{r}, t-t_{3}-t_{2}\right) E_{1}\left(\mathbf{r}, t-t_{3}-t_{2}-t_{1}\right)
\end{aligned}
$$

where $R^{(3)}$ is the third order response function given by

$$
R^{(3)}\left(t_{3}, t_{2}, t_{1}\right)=\left(\frac{i}{\hbar}\right)^{3}\left\langle V_{\nu_{4}}\left|G\left(t_{3}\right) V_{\nu_{3}} G\left(t_{2}\right) V_{\nu_{2}} G\left(t_{1}\right) V_{\nu_{1}}\right| \rho_{0}\right\rangle
$$

The response function is a useful quantity that describes the full time-dependent, microscopic state of the system for any set of three input pulses arriving at times $t_{i}$. For a given Hamiltonian, the response function can be directly calculated for the purposes of computational modelling. The system is initially in the equilibrium state 
$\rho_{0}$. The dipole operator $V_{\nu_{i}}$ modifies the density matrix following interaction with the $i^{t h}$ pulse, and the field-free molecular time evolution for an interval $t_{i}$ is given by the Green's function $G\left(t_{i}\right)$. The final interaction $V_{\nu_{4}}$ is the signal mode giving the final response.

In the ideal case, we assume impulsive (delta function) pulses, such that the time intervals are well defined and well-ordered, and the pulses are much shorter than the relevant molecular dynamics which we are attempting to measure.

To facilitate understanding and computation of the signals, the incoming electric field can be expanded in modes:

$$
E(\mathbf{r}, t)=\sum_{j}\left[\varepsilon_{j}(t) \exp \left(i \mathbf{k}_{j} \cdot \mathbf{r}-i \omega_{j} t\right)+\varepsilon_{j}^{*}(t) \exp \left(-i \mathbf{k}_{j} \cdot \mathbf{r}+i \omega_{j} t\right)\right]
$$

where $\varepsilon(t)$ is the temporal pulse envelope and the summation index $j$ is over the pulse number (input pulses 1-3 and signal $s$ ). In NMR, the pulse wavelengths (typically on the order of meters) are much larger than the sample size, so the condition $k r \ll 1$ means that the signal is essentially isotropic in space for these experiments. Because of this, the signal is generally selected via phase-cycling in NMR [4]. For the visible and near-IR wavelengths (400-800 $\mathrm{nm}$ ) used in 2DES experiments, however, the opposite approximation is true: $k r \gg 1$. Therefore, in 2DES experiments, the signals are highly directional, and the signals related to specific energy pathways are chosen through a phase-matching condition. The response function can then be expanded as

$$
R^{(3)}\left(t_{3}, t_{2}, t_{1}\right)=\left(\frac{i}{\hbar}\right)^{3} \sum_{l} R_{l}^{(3)}\left(t_{3}, t_{2}, t_{1}\right) \exp \left(i \mathbf{k}_{l} \cdot \mathbf{r}-i \omega_{l} t\right)
$$

The $R_{l}$ are the response function components associated with each pathway in the signal. Because there are three input pulses, and each pulse can interact with either the ket or the bra (positive or negative frequency), there are $2^{3}=8$ different signals over which the index $l$ iterates. The phase and frequency matching conditions for 
these are

$$
\begin{aligned}
& \mathbf{k}_{l}= \pm \mathbf{k}_{1} \pm \mathbf{k}_{2} \pm \mathbf{k}_{3} \\
& \omega_{l}= \pm \omega_{1} \pm \omega_{2} \pm \omega_{3}
\end{aligned}
$$

In general, the cases in which all the frequency components have the same sign are highly oscillatory and do not contribute largely to the signal and can be neglected by employing the rotating wave approximation. The dominant signals of interest in 2DES are usually referred to as the photon echo, or rephasing, signal $\left(\mathbf{k}_{s}=-\mathbf{k}_{1}+\mathbf{k}_{2}+\mathbf{k}_{3}\right)$ and the non-rephasing signal, sometimes called the "virtual echo" $\left(\mathbf{k}_{s}=+\mathbf{k}_{1}-\mathbf{k}_{2}+\mathbf{k}_{3}\right)$. The terminology comes from the fact that the phase evolves at conjugate frequencies during the two coherence periods in the rephasing signal but not in the non-rephasing. Thus the former is able to reform into an "echo", while phase evolution in the latter can only continue in the same direction. Contributions to the signal in directions that do not match the phase-matching condition will vanish due to a randomness in the phase [4]. It is common practice in atomic and molecular optics to graphically represent the various signal pathways associated with the field-matter interactions and density matrix field-free evolution periods [9]. The following section will explore a specific graphical representation commonly used in 2D spectroscopy.

\subsection{Double-sided Feynman Diagrams}

While the mathematical notation can get complicated, and a full treatment of the high rank tensors involved in the complete calculations can make bookkeeping difficult, there is a simple tool for graphically representing the various pathways of evolution of the density matrix. It is common in 2DES and 2DIR to use doublesided Feynman diagrams to represent and guide the interpretation of the underlying calculations $[4,10]$. 
The Feynman diagrams for the rephasing and non-rephasing pathways are shown in Figure 2.1 along with the corresponding energy level diagrams. The two vertical lines represent the ket (left) and bra (right) side of the density matrix. Time is positive along the vertical axis, from bottom to top. Each interaction with the radiation field can affect either the ket or the bra and is represented by an arrow with its head or tail intersecting the appropriate vertical line. Arrows pointing to the right represent a contribution of $\varepsilon_{j}(t) \exp \left(i \mathbf{k}_{j} \cdot \mathbf{r}-i \omega_{j} t\right)$ to the polarization and are associated with a positive wavevector $\mathbf{k}_{j}$. Arrows pointing to the left represent a contribution of $\varepsilon_{j}^{*}(t) \exp \left(-i \mathbf{k}_{j} \cdot \mathbf{r}+i \omega_{j} t\right)$ to the polarization and are associated with a negative wavevector $-\mathbf{k}_{j}[11]$. Incoming arrows represent a photon absorption (negative frequency $-\omega_{j}$ ), while outgoing arrows represent a photon emission (positive frequency $\left.\omega_{j}\right)$. Each diagram has an overall sign of $(-1)^{n}$, where $n$ is the number of interactions from the right (bra) side of the diagram. This is a consequence of the fact that operators acting to the right in a commutator carry a minus sign. [1]

Note that while each signal is defined by the phase-matching diagrams, there can be several underlying processes contributing to each signal. The phase matching conditions ensure that the signals are spatially separable under partially or wholly non-collinear beam geometries, but the underlying contributions cannot be separated spatially. In many cases, however, these will be spectrally distinct when plotted in the 2D frequency-frequency map.

\subsection{Feynman Diagrams and the Density Matrix}

In the general case of pathways involved in 2DES, the first pulse will interact with either the ket or the bra. A single pulse interaction places the sample in a coherence, represented by an off-diagonal element in the density matrix $\rho_{i j}$, which oscillates at the frequency of absorption during the coherence time $\left(t_{1}\right)$ as illustrated in Figure 2.2. This coherence decays rapidly (within several hundred fs in the condensed phase 

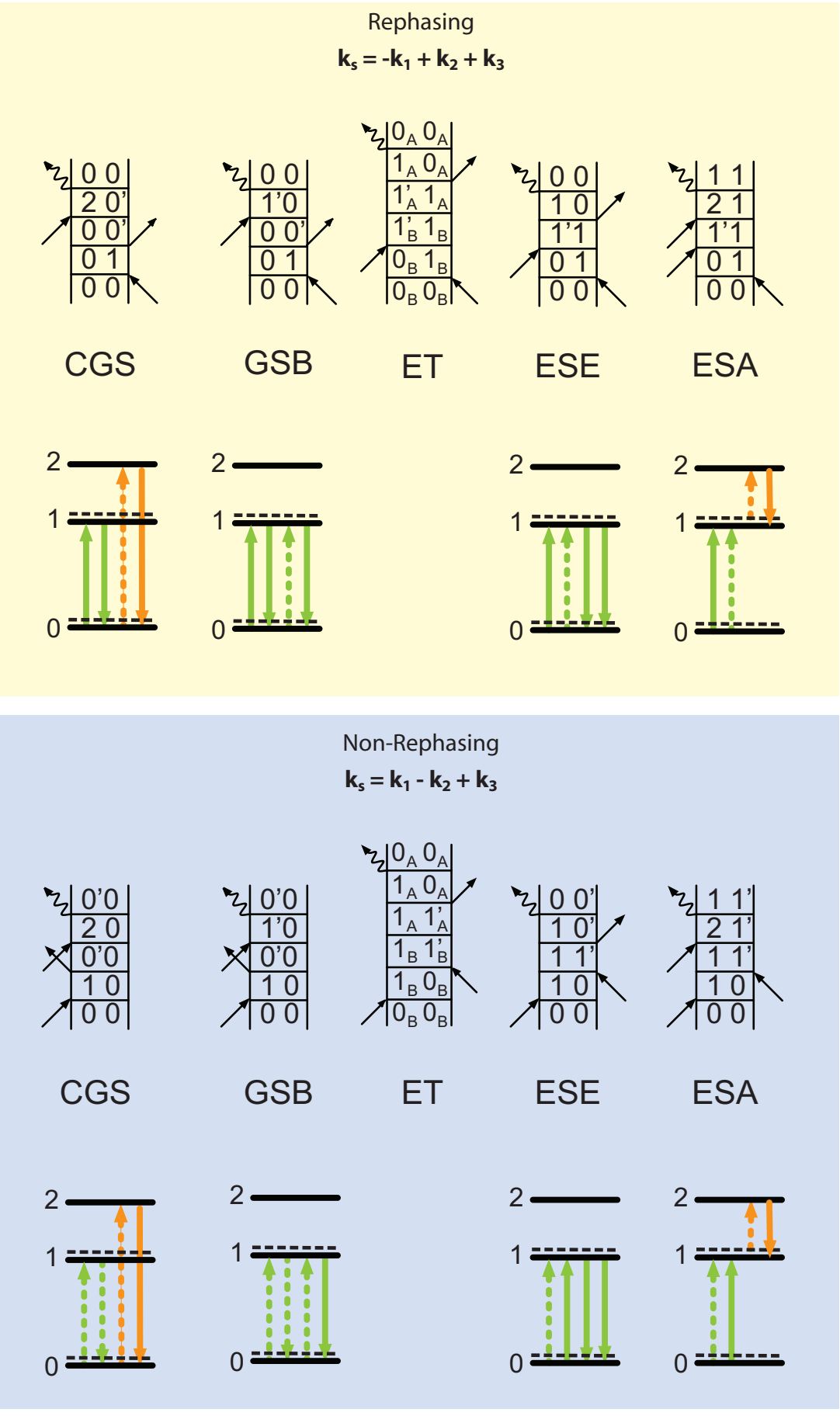

Figure 2.1: Double-sided Feynman diagrams for the rephasing and non-rephasing pathways. These include contributions from ground state bleach (GSB), excited state emissions (ESE) and excited state absorption (ESA), as well as diagrams related to features with common ground states (CGS) or energy transfer (ET). The energy levels below show the pulse interactions corresponding to each Feynman diagram. Dotted (solid) lines denote interactions with the ket (bra). 
samples we study) due to dephasing processes. In the Feynman diagram, this is represented by a difference in states between the ket and the bra: $|0\rangle\langle 1|$ or $|1\rangle\langle 0|$. After free evolution with the Green function $G\left(t_{1}\right)$, the second pulse interaction creates a population in either the ground or excited state: $|0\rangle\left\langle 0^{\prime}\right|$, or $|1\rangle\left\langle 1^{\prime}\right|$, respectively. This corresponds to a diagonal element in the density matrix $\rho_{i i^{\prime}}$. The prime (') notation on the states $\left|0^{\prime}\right\rangle$ and $\left|1^{\prime}\right\rangle$ indicate that the ket and the bra may be on different vibrational sublevels of the same electronic state [12]. The time between the second and third pulses $\left(t_{2}\right)$ is often called the "population time" - it is during this long-lived period that most of the interesting system dynamics occur, including Stokes shift, energy transfer, charge separation, conformational changes, and quenching. It is also possible that the sample could be in a vibrational coherence on the ground or excited state (see PERY data in Chapter 2.7). The third pulse again creates a coherence in the sample. Depending on the frequency of the interaction and available manifold of states, this coherence can be between the ground and first excited state or between the first and second excited states, in the case of excited state absorption. The signal field radiates at a time $t_{3}$ after the third pulse in a phase matched direction determined by the pathway involved. Figure 2.2 shows the density matrix elements involved in an example 2DES signal from a rephasing pathway.

\subsection{Types of Signal Contributions}

The general case discussed above includes several possible contributions to the signal for each phase-matched pathway. The Feynman diagrams for these contributions are given in Figure 2.1, while their manifestation in 2D spectra is discussed in the next section.

We will first consider the simple case of a single molecular species with a threelevel manifold, consisting of the ground state $|0\rangle$, a first excited state $|1\rangle$ and a second excited state $|2\rangle$. There are three contributions of interest to us: ground state bleach 


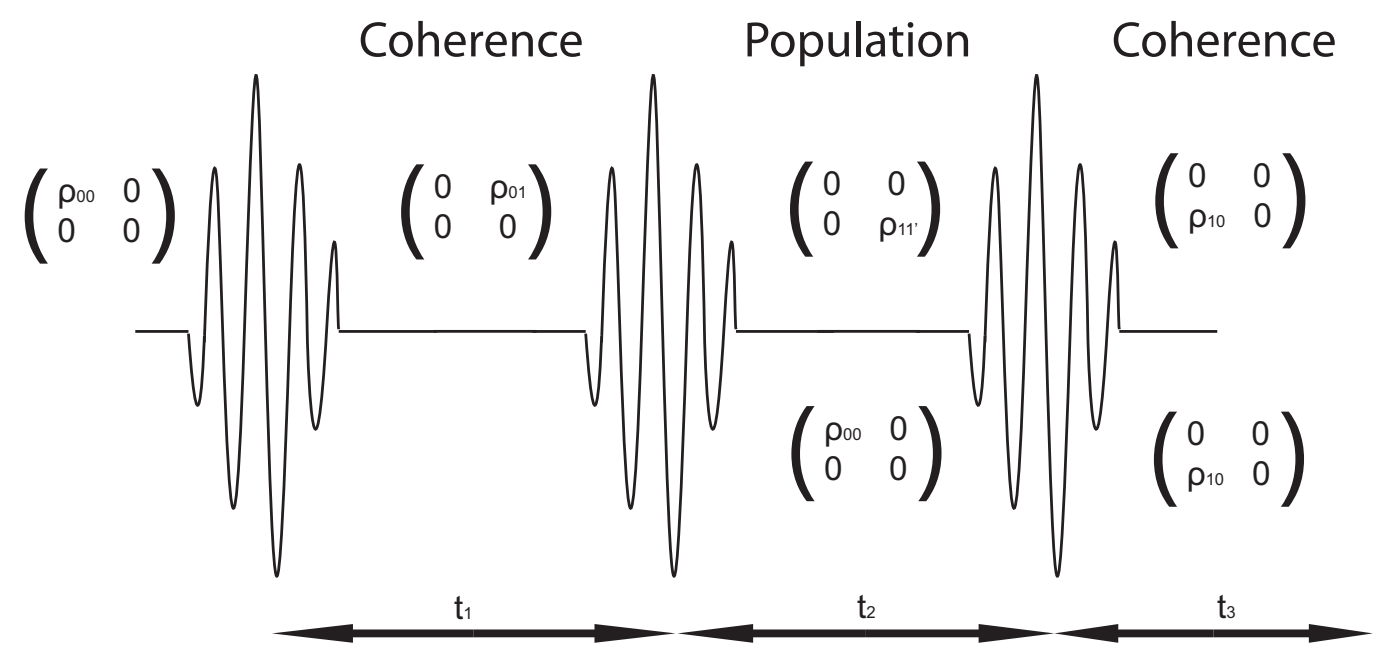

Figure 2.2: A diagram of the density matrix elements involved during coherence and population times for a rephasing signal. This figure is adapted from [13].

(GSB), excited state emission (ESE), and excited state absorption (ESA). All three of these will begin the same, with a coherence between the ground and first excited state, excited on either the bra (rephasing) or ket (non-rephasing). However, the second pulse can create a population in either the ground state or first excited state. If the population is in the ground state during $t_{2}$, the third pulse will bleach the ground state (GSB), removing its absorption band from the transient spectrum.

If the population is in the first excited state during $t_{2}$, one of two things can happen. In the case of excited state emission, the third pulse will knock the sample back into a coherence between the ground and first excited state, creating a regular stimulated emission event. ESE and GSB are spectrally indistinguishable and are therefore generally discussed together. It is also possible, if the second excited state is accessible within the pulse bandwidth, that the third pulse will create a coherence between the first and second excited states. This type of event is called ESA, and the signal frequency will contain information on the transition between the first and second excited as well as between the ground and first excited states. By the rules for constructing Feynman diagrams given above, the ESA signal will carry a negative 
sign, while the GSB and ESE will be positive. In samples with largely harmonic energy levels, the ESA will spectrally overlap the other two signals, and they can partially or completely cancel one another. Therefore, it is only the anharmonicities which shift this signal and allow study of ESA in this technique [13].

There is a fourth type of signal contribution that can show up in the case where energy transfer between two exciton states is allowed. In this case, a population in the excited state can transfer its excitation during the population time $t_{2}$ to a separate chromophore or exciton state. Given two exciton states $\left|1_{a}\right\rangle$ and $\left|1_{b}\right\rangle$ with allowed energy transfer, the transition during $t_{2}$ will change the density matrix from the state $\left|1_{a}\right\rangle\left\langle 1_{a}^{\prime}\right|$ to $\left|1_{b}\right\rangle\left\langle 1_{b}^{\prime}\right|$. This energy transfer (ET) signal gives rise to a distinct feature in the 2D spectrum known as a cross-peak, which is explicitly distinguishable in a 2D spectrum but is often mixed ambiguously into other signals in different spectroscopy techniques.

\section{$2.6 \quad 2 \mathrm{D}$ spectra}

The $2 \mathrm{D}$ spectrum contains information about both coherence periods: $t_{1}$ and $t_{3}$. Generally, $t_{1}$ is scanned in small steps and Fourier transformed to a frequency axis $\omega_{1}$. This axis contains information about the transition to the first excited state and can be considered as a label of the initial excitation frequency. In most cases, the delay $t_{3}$ is measured directly in the frequency domain $\omega_{3}$ to facilitate easier data collection. $\omega_{3}$ contains frequency information about the second coherence, which is related to the frequency of the third pulse, which probes the state of the sample following the dynamics during the $t_{2}$ period. Therefore, the $2 \mathrm{D}$ spectrum acts as a correlation map, wherein the $\omega_{1}$ axis can be thought of as the "excitation axis" and $\omega_{3}$ is considered the "detection axis". The $t_{2}$ delay is fixed for a given spectrum, so each $2 \mathrm{D}$ spectrum is like a snapshot of the sample state at a specific value of $t_{2}$. To analyze kinetics, several spectra must be taken for different values of $t_{2}$. A complete scan over $t_{2}$ will give a 3D 
spectrum $\tilde{S}\left(\omega_{1}, t_{2}, \omega_{3}\right)$. When all the phase-matched signals and tensor components are recorded this enables measurements of the complete $3^{\text {rd }}$ order response function [14]. The treatment and analysis of the 3D spectrum will be discussed in Chapter 3.8, where these measurements are applied to the $\mathrm{RC}$.

To illustrate some of the types of signals observed in a 2DES experiment, several simple cases are depicted in Figure 2.3. A cartoon 2D spectrum is shown in Figure 2.3(a) for a case of two uncoupled two-level chromophores with different transition frequencies, as indicated by the energy level diagrams. A peak on the diagonal indicates GSB and ESE signals for which the absorption and emission frequencies are the same. The linewidth along the diagonal indicates the inhomogeneous broadening, while the antidiagonal width is the homogeneous linewidth [12]. For early $t_{2}$, the spectra tend to be very inhomogeneously broadened, indicating a high degree of correlation between the excitation and detection frequencies. At longer values of $t_{2}$, these peaks will often become rounder, indicating a lack of correlation, which can be thought of as a "loss of memory" of the initial state. This loss of correlation is often termed "spectral diffusion" and arises due to interactions between the chromophore and environment that introduce frequency fluctuations [12].

Figure 2.3(b) shows another cartoon spectrum. This time, we have included a second excited state for each chromophore and allowed weak coupling between the chromophores, giving rise to incoherent (Förster) energy transfer between the first excited states of the two chromophores. For anharmonic systems, the ESA peak is often shifted below the main diagonal peak, as shown. The energy transfer event shows up as an off-diagonal cross-peak in the spectrum. The coordinates of the cross peak $\left(\omega_{1}, \omega_{3}\right)$ reveal the transition frequencies of the two states involved, and the $t_{2}$ dependence of the cross peak amplitude reveals the energy transfer dynamics. For systems of more than two chromophores, one will often see several cross-peaks in different locations of the spectrum. This provides an unambiguous examination 
of different energy transfer events between distinct states. This information is not easily available with other spectroscopy techniques. For example, transient absorption studies effectively integrate over the excitation axis since the $t_{1}$ delay is not scanned.

Figure 2.3(c) depicts a simple case of strong (excitonic) coupling between two chromophores. In this case their energy levels are shifted due to their strong coupling, and the presence of a common ground state gives rise to cross-peaks. These crosspeaks will be present at all values of $t_{2}$ and their existence at $t_{2}=0$ when other processes such as energy transfer have not yet occurred indicate excitonic coupling. Similarly well-separated vibrational levels on the same electronic state can give rise to cross-peaks. In both these cases the corresponding Feynman diagram resembles that of GSB, where the state accessed by the third pulse excites a different excitonic level in the first case or a different vibrational state in the latter. It has also been recently demonstrated in the IR that coherence transfer can occur in some vibrational systems [15].

\subsection{A Note on Modelling}

For complex multichromophoric samples such as the photosynthetic reaction centers presented later in this thesis, the interpretation of a $2 \mathrm{D}$ spectrum is difficult. Degeneracies in energy levels between different chromophores can lead to overlap of features which make the line widths and dynamics complicated and harder to discern. In addition to fitting and analysis of experimental 2D spectra, it is useful to treat the data in a phenomenological manner through comparison to theoretical models of the systems under study. By beginning with knowledge of the chromophore stoichiometry and dipole moments of the individual chromophores and figuring in such factors as orientation of species and interchromophore distances, a Hamiltonian can be constructed for evolution of the density matrix, and $2 \mathrm{D}$ spectra can be built up from a theoretical basis. Because of the inherent computational cost of performing 
(a)
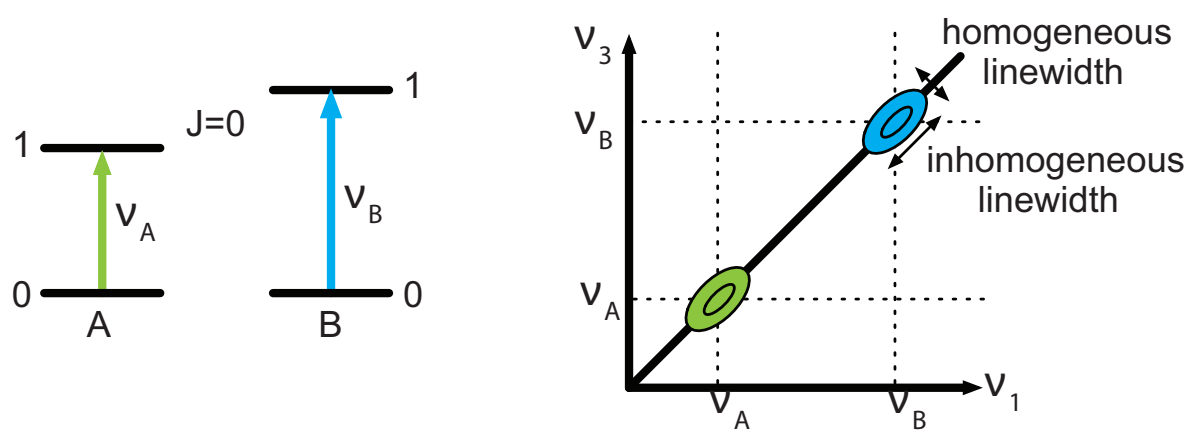

(b)
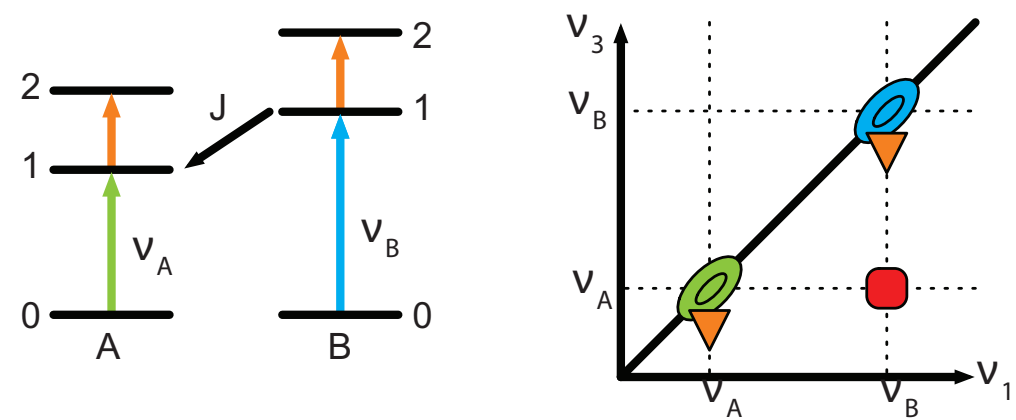

(c)
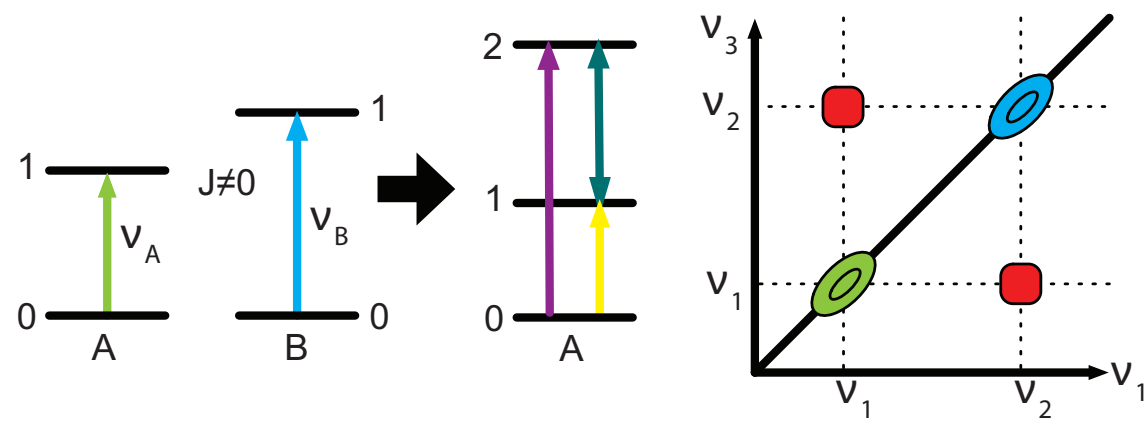

Figure 2.3: Energy levels diagrams for different coupling cases with cartoon representations of the 2DES spectra. (a) shows two uncoupled two-level chromophores, and (b) gives two three-level chromophores with weak coupling between the first excited states. (c) shows the excitonic splitting of energy levels in a strong coupling case. 
many-body quantum calculations, each model must begin with some assumptions and approximations which can be checked and validated by experimental findings. In this way, the experiment and models reinforce each other.

In some cases, parameters corresponding to physical observables which are difficult to measure directly in the experimental data can be tweaked in the model until the best agreement between model and experiment is reached. In other cases, the increased dimensionality of the 2D data can help researchers compare the relative validity of two different models which produce similar linear absorption data [12].

In this thesis, comparisons will be made between kinetic parameters extracted from our 2D data and measurable parameters from models, most of which have been built up by comparison to other spectroscopy methods. Much can be learned from this sort of treatment, and the validity of different prevailing models can be compared against the information content of the $2 \mathrm{D}$ spectra presented here. Ideally, these models will be adapted to produce theoretical 2D spectra for a more thorough comparison. This is indeed a goal and area of ongoing study in our lab. However, the modelling of $2 \mathrm{D}$ spectral lineshapes is by no means a trivial task and is beyond the scope of the present work. A full simulation of $2 \mathrm{D}$ specra for the RC shall not be covered here and will instead be the subject of forthcoming papers and dissertations from the Ogilvie group.

A brief sketch of the future modelling process is shown in Figure 2.4 below. A set of generalized coordinates $\mathbf{Q}$ provides a physical description of the system. Beginning with a basis of the individual chromophores, the Hamiltonian $\tilde{H}_{S}^{e}$ gives the individual site energies and couplings between chromophores. These couplings are inferred from crystal structures, although the current spatial resolution limits the precision with which these can be obtained. Weak coupling may be treated perturbatively in some cases, but intermediate to strong coupling can give rise to delocalized exciton states with energies significantly different from the site energies. The Hamiltonian is then 
diagonalized to provide an energy eigenstate basis. The new Hamiltonion, $\mathbf{H}_{S}$, maps onto the peak positions of the $2 \mathrm{D}$ spectrum at $t_{2}=0$, with cross-peaks indicating coupling between exciton states. As mentioned in Chapter, several exciton models exist for the RC and will form a starting point for our modelling.

In condensed phase systems, the system is constantly interacting with the surrounding bath, which can cause changes and fluctuations to the energy eigenstates. In proteins, this "bath" is highly heterogeneous and dynamic. The bath is modelled with the Hamiltonian $\mathbf{H}_{B}$, and the system-bath coupling Hamiltonian $\mathbf{H}_{S B}$ contains the information on the system-bath interaction. In the simplest treatment, the bath is treated as a system of harmonic oscillators with appropriate frequencies and damping parameters. In order to simplify the calculation, researchers will generally average over all but a few of the bath degrees of freedom [1]. From this description of the system and bath, the third order response function $R^{(3)}$ can be calculated, and a 2D spectrum can be generated. Through comparison to experimental spectra and other considerations, the parameters can then be adjusted and the spectra corrected through an iterative approach.

Modelling of 2DIR data has reached a high level of sophistication, where input from molecular dynamics (MD) simulations can be used to predict 2D spectra $[18,19$, 20, 21, 22, 23, 24, 25, 26, 27, 28, 29, 30, 31, 32, 33, 34, 35, 36, 37, 38]. The modelling efforts for 2DES are substantially less advanced, due to the high computational cost of calculating electronic structure of large systems in the condensed phase. It remains an area of active research to determine what minimum degrees of freedom need fully quantum mechanical treatment and what other degrees of freedom can be treated classically $[27,28,39,40,41,10,38,42,43,44,45]$. It is our hope that 2DES will further the development of mixed quantum/classical models of complex condensed phase systems and that they will in turn help reveal the dynamics and electronic structure underlying 2D lineshapes. 


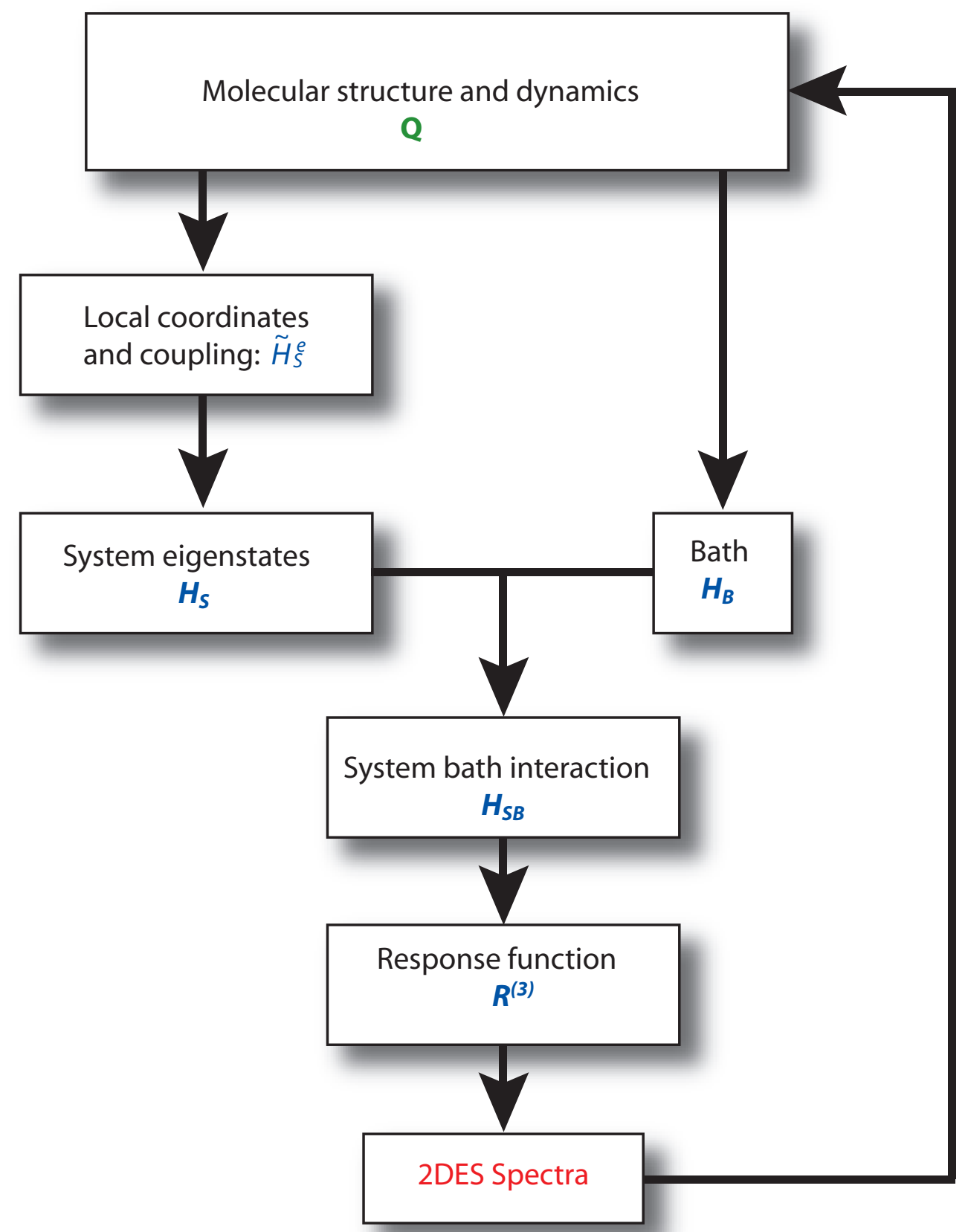

Figure 2.4: This flowchart describes the general process for modelling a 2DES spectrum on a given system. This figure is modelled on [16, 17, 13]. 


\section{Bibliography}

[1] Shaul Mukamel. Principles of Nonlinear Optical Spectroscopy. Oxford University Press, 1995.

[2] Minhaeng Cho. Two-Dimensional Optical Spectroscopy. CRC Press, 2009.

[3] L Allen and JH Eberly. Optical Resonances and Two-Level Atoms. Wiley, 1975.

[4] W. Zhuang, T. Hayashi, and S. Mukamel. Coherent multidimensional vibrational spectroscopy of biomolecules: Concepts, simulations, and challenges. Angewandte Chemie-international Edition, 48(21):3750-3781, 2009.

[5] R. F. Loring and S. Mukamel. Selectivity in coherent transient raman measurements of vibrational dephasing in liquids. Journal of Chemical Physics, 83(5):2116-2128, 1985.

[6] A. V. Pisliakov, T. Mancal, and G. R. Fleming. Two-dimensional optical threepulse photon echo spectroscopy. ii. signatures of coherent electronic motion and exciton population transfer in dimer two-dimensional spectra. Journal of Chemical Physics, 124, 2006.

[7] B Donovan, LA Walker, CF Yocum, and RJ Sension. Transient absorption studies of the primary charge separation in photosystem II. Journal of Physical Chemistry, 100(5):1945-1949, FEB 11996.

[8] M. G. Muller, M. Hucke, M. Reus, and A. R. Holzwarth. Annihilation processes in the isolated d1-d2-cyt-b559 reaction center complex of photosystem ii. an intensity-dependence study of femtosecond transient absorption. Journal of Physical Chemistry, 100(22):9537-9544, May 1996.

[9] P. R. Berman and W. E. Lamb. Theory of collision effects on line shapes using a quantum-mechanical description of the atomic center-of-mass motion - application to lasers. i. Physical Review A-general Physics, 2(6):2435-2454, 1970.

[10] M. H. Cho. Coherent two-dimensional optical spectroscopy. Chemical Reviews, 108(4):1331-1418, 2008.

[11] CJ Bordé. Advances in Laser Spectroscopy, pages 1-70. Plenum Publishing Corporation, 1983. 
[12] D. M. Jonas. Two-dimensional femtosecond spectroscopy. Annual Review of Physical Chemistry, 54:425-463, 2003.

[13] J. P. Ogilvie and K. J. Kubarych. Multidimensional electronic and vibrational spectroscopy: An ultrafast probe of molecular relaxation and reaction dynamics. Advances In Atomic, Molecular, and Optical Physics, Vol 57, 57:249-321, 2009.

[14] P. Hamm. Three-dimensional-ir spectroscopy: Beyond the two-point frequency fluctuation correlation function. Journal of Chemical Physics, 124(12), 2006.

[15] M. J. Nee, C. R. Baiz, J. M. Anna, R. McCanne, and K. J. Kubarych. Multilevel vibrational coherence transfer and wavepacket dynamics probed with multidimensional ir spectroscopy. Journal of Chemical Physics, 129(8):084503, August 2008 .

[16] M. Khalil, N. Demirdoven, and A. Tokmakoff. Obtaining absorptive line shapes in two-dimensional infrared vibrational correlation spectra. Physical Review Letters, 90(4), 2003.

[17] M. Khalil, N. Demirdoven, and A. Tokmakoff. Coherent 2d ir spectroscopy: Molecular structure and dynamics in solution. Journal of Physical Chemistry A, 107:5258-5279, 2003.

[18] C. J. Fecko, J. D. Eaves, J. J. Loparo, A. Tokmakoff, and P. L. Geissler. Ultrafast hydrogen-bond dynamics in the infrared spectroscopy of water. Science, 301(5640):1698-1702, September 2003.

[19] J. D. Eaves, J. J. Loparo, C. J. Fecko, S. T. Roberts, A. Tokmakoff, and P. L. Geissler. Hydrogen bonds in liquid water are broken only fleetingly. Proceedings of the National Academy of Sciences of the United States of America, 102(37):13019-13022, September 2005.

[20] J. D. Eaves, A. Tokmakoff, and P. L. Geissler. Electric field fluctuations drive vibrational dephasing in water. Journal of Physical Chemistry A, 109(42):94249436, October 2005.

[21] J. Dreyer. Density functional theory simulations of two-dimensional infrared spectra for hydrogen-bonded acetic acid dimers. International Journal of Quantum Chemistry, 104(5):782-793, September 2005.

[22] J. Dreyer. Hydrogen-bonded acetic acid dimers: Anharmonic coupling and linear infrared spectra studied with density-functional theory. Journal of Chemical Physics, 122(18), 2005.

[23] J. L. Skinner, H. C. Andersen, and M. D. Fayer. Theory of photon-echoes from a pair of coupled 2 level systems - impurity dimers and energy-transfer in molecular-crystals. Journal of Chemical Physics, 75(7):3195-3202, 1981. 
[24] J. B. Asbury, T. Steinel, C. Stromberg, S. A. Corcelli, C. P. Lawrence, J. L. Skinner, and M. D. Fayer. Water dynamics: Vibrational echo correlation spectroscopy and comparison to molecular dynamics simulations. Journal of Physical Chemistry A, 108(7):1107-1119, 2004.

[25] S. A. Corcelli, C. P. Lawrence, J. B. Asbury, T. Steinel, M. D. Fayer, and J. L. Skinner. Spectral diffusion in a fluctuating charge model of water. Journal of Chemical Physics, 121(18):8897-8900, November 2004.

[26] J. R. Schmidt, S. T. Roberts, J. J. Loparo, A. Tokmakoff, M. D. Fayer, and J. L. Skinner. Are water simulation models consistent with steady-state and ultrafast vibrational spectroscopy experiments? Chemical Physics, 341(1-3):143157, November 2007.

[27] G. Hanna and E. Geva. Vibrational energy relaxation of a hydrogen-bonded complex dissolved in a polar liquid via the mixed quantum-classical lionville method. Journal of Physical Chemistry B, 112(13):4048-4058, April 2008.

[28] G. Hanna and E. Geva. Computational study of the one and two dimensional infrared spectra of a vibrational mode strongly coupled to its environment: Beyond the cumulant and condon approximations. Journal of Physical Chemistry B, 112(41):12991-13004, October 2008.

[29] D. Abramavicius, W. Zhuang, and S. Mukamel. Peptide secondary structure determination by three-pulse coherent vibrational spectroscopies: A simulation study. Journal of Physical Chemistry B, 108(46):18034-18045, November 2004.

[30] V. Chernyak, W. M. Zhang, and S. Mukamel. Multidimensional femtosecond spectroscopies of molecular aggregates and semiconductor nanostructures: The nonlinear exciton equations. Journal of Chemical Physics, 109(21):9587-9601, December 1998.

[31] S. Mukamel, A. Piryatinski, and V. Chernyak. Semiclassical simulations of multidimensional raman echoes. Journal of Chemical Physics, 110(3):1711-1725, January 1999.

[32] A. Piryatinski, S. Tretiak, V. Chernyak, and S. Mukamel. Simulations of twodimensional femtosecond infrared photon echoes of glycine dipeptide. Journal of Raman Spectroscopy, 31(1-2):125-135, January 2000.

[33] A. Piryatinski, V. Chernyak, and S. Mukamel. Two-dimensional correlation spectroscopies of localized vibrations. Chemical Physics, 266(2-3):311-322, May 2001.

[34] C. Scheurer, A. Piryatinski, and S. Mukamel. Signatures of beta-peptide unfolding in two-dimensional vibrational echo spectroscopy: A simulation study. Journal of the American Chemical Society, 123(13):3114-3124, April 2001. 
[35] A. Piryatinski, S. A. Asher, and S. Mukamel. Real space analysis of excitonic interactions and coherence length in helical aggregates. Journal of Physical Chemistry A, 106(14):3524-3530, April 2002.

[36] A. M. Moran, S. M. Park, and S. Mukamel. Infrared photon echo signatures of hydrogen bond connectivity in the cyclic decapeptide antamanide. Journal of Chemical Physics, 118(22):9971-9980, June 2003.

[37] A. M. Moran, J. Dreyer, and S. Mukamel. Ab initio simulation of the two-dimensional vibrational spectrum of dicarbonylacetylacetonato rhodium(i). Journal of Chemical Physics, 118(3):1347-1355, January 2003.

[38] W. Zhuang, D. Abramavicius, T. Hayashi, and S. Mukamel. Simulation protocols for coherent femtosecond vibrational spectra of peptides. Journal of Physical Chemistry B, 110(7):3362-3374, February 2006.

[39] G. R. Fleming and M. H. Cho. Chromophore-solvent dynamics. Annual Review of Physical Chemistry, 47:109-134, 1996.

[40] M. H. Cho and G. R. Fleming. The integrated photon echo and solvation dynamics. ii. peak shifts and two-dimensional photon echo of a coupled chromophore system. Journal of Chemical Physics, 123(11):114506, September 2005.

[41] M. H. Cho, T. Brixner, I. Stiopkin, H. Vaswani, and G. R. Fleming. Two dimensional electronic spectroscopy of molecular complexes. Journal of the Chinese Chemical Society, 53(1):15-24, February 2006.

[42] M. F. Gelin, D. Egorova, and W. Domcke. Efficient method for the calculation of time- and frequency-resolved four-wave mixing signals and its application to photon-echo spectroscopy. Journal of Chemical Physics, 123(16), 2005.

[43] D. Egorova, M. F. Gelin, and W. Domcke. Analysis of vibrational coherences in homodyne and two-dimensional heterodyne photon-echo spectra of nile blue. Chemical Physics, 341(1-3):113-122, 2007.

[44] D. Egorova, M. F. Gelin, and W. Domcke. Analysis of cross peaks in twodimensional electronic photon-echo spectroscopy for simple models with vibrations and dissipation. Journal of Chemical Physics, 126(7), 2007.

[45] D. Abramavicius, B. Palmieri, D. V. Voronine, F. Sanda, and S. Mukamel. Coherent multidimensional optical spectroscopy of excitons in molecular aggregates; quasiparticle versus supermolecule perspectives. Chemical Reviews, 109(6):2350 2408, 2009. 


\section{CHAPTER III}

\section{Experimental Methods}

In this chapter, the experimental methods and apparatus used in obtaining twodimensional electronic spectra are described in detail. The first section briefly describes the commercially-built laser oscillator and regenerative amplifier. Section 3.2 details the design and tuning of our home-built non-collinear optical parametric amplifiers (NOPAs). Following that, there is some discussion on pulse compression with a prism compressor. Sections 3.5 and 3.6 present two different experimental methods for 2DES, both implemented in our lab, using a diffractive optic and an acousto-optic pulse shaper, respectively, to create the pair of pump pulses. The relative strengths of each method and a guide for situations where one may be advantageous over the other are presented in Section 3.1. Finally, section 3.10 presents a brief discussion regarding the use of a continuum probe pulse in obtaining data over a broader bandwidth.

\subsection{Laser Source}

The laser source consists of a mirror-dispersion-controlled titanium:sapphire oscillator (Femtosource Synergy) [1]. The oscillator operates at a repetition rate of 75 $\mathrm{MHz}$ and emits a self-mode-locked pulse of $10 \mathrm{fs}$ duration and bandwidth greater than $100 \mathrm{~nm}$ centered near $800 \mathrm{~nm}$. Typical mode-locked output powers range between 550-700 mW. 
A small fraction of the oscillator output is used to seed a regenerative amplifier (Spectra Physics Spitfire Pro). A Faraday isolator precludes the possibility of backward reflections from disrupting the mode-lock in the oscillator. The regenerative amplifier (regen) employs a method known as chirped pulse amplification [2]. The peak powers associated with amplifying the transform-limited seed pulse to the desired output would exceed the damage threshold of the Ti:Sapphire crystal in the amplifier cavity. However, by stretching the seed pulse with a grating stretcher to a duration $>2$ ps, the instantaneous peak power in the crystal is greatly reduced, and high gain amplification can be achieved without risk of damage. The pulse is stretched in a grating stretcher before entering the cavity. A Pockels cell selects pulses at a rate of $1 \mathrm{kHz}$. In the cavity, the seed pulse is amplified in a Ti:sapphire crystal by a 7 Watt Q-switched Nd:Yag pump laser (Spectra Physics Empower). Pulses make several round trips in the cavity before they are coupled out by a second Pockels cell. The amplified chirped pulses then enter a grating compressor where they are compressed to near the transform limit. The $1 \mathrm{~mJ}$ output pulses have a duration of 35 fs at $800 \mathrm{~nm}$ with a $1 \mathrm{kHz}$ repetition rate. The grating separation can also be set to precompensate the output pulses for maximum compression later in the setup, so as to achieve higher efficiency in the parametric amplification step described in the following section.

\subsection{NOPAs}

The output from the regen is split between two home-built non-collinear optical parametric amplifiers (NOPAs) [3]. Within each NOPA, a $4 \%$ fraction of the beam is focused with a $5 \mathrm{~cm}$ lens into a $1 \mathrm{~mm}$ thick sapphire window. Self-phase modulation (SPM) in the sapphire window produces a chirped white light continuum pulse which is used as the NOPA seed. The rest of the beam is frequency doubled in a $0.5 \mathrm{~mm}$ Beta-barium borate (BBO) crystal to $400 \mathrm{~nm}$. The continuum seed and $400 \mathrm{~nm}$ 
pump are overlapped in a $2 \mathrm{~mm}$ BBO crystal, placed $2-3 \mathrm{~cm}$ behind the pump focus to avoid damage. The angle between seed and pump beams is generally set to $3-5^{\circ}$ and is designed to take advantage of the spatial walk-off in order to obtain maximal spatial overlap throughout the crystal.

The beams undergo a type I difference frequency process in the BBO, producing an output beam theoretically tunable to any frequency contained within the continuum pulse. The process is governed by the equation

$$
f_{p}=f_{s}+f_{i}
$$

where $f_{s}$ is the signal frequency, $f_{p}$ is the pump frequency, and $f_{i}$ is the frequency of the idler pulse that serves to conserve energy and momentum. Figure 3.1 shows (a) the alignment of seed an pump pulses into the BBO crystal, (b) the phase-matching condition for pump, signal and idler, and (c) a cartoon of the beams immediately after the picture, including a parametric fluorescence ring from spontaneous difference frequency generation not aligned with the seed.

A delay stage in the continuum pulse path allows for adjustment of the temporal overlap between different frequency components of the chirped seed pulse and the pump, so that the center frequency can be adjusted with a micrometer. The stage is adjusted along with the alignment of the seed pulse to select the desired spectrum. Adjusting the angle of the $\mathrm{BBO}$ relative to the two input pulses can optimize the spectrum for different frequency ranges. The NOPA pulses can be tuned from $\sim 480$ - $700 \mathrm{~nm}$ center wavelength, with bandwidths up to $70 \mathrm{~nm}$. Figure 3.2 gives a series of spectra from our NOPA, demonstrating the broad tunability we achieve in our setup. 
(a)

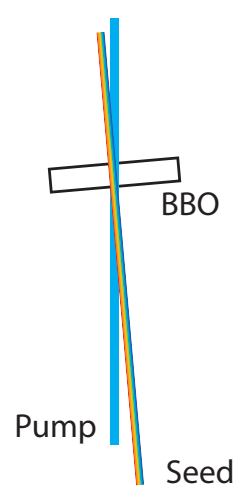

(b)

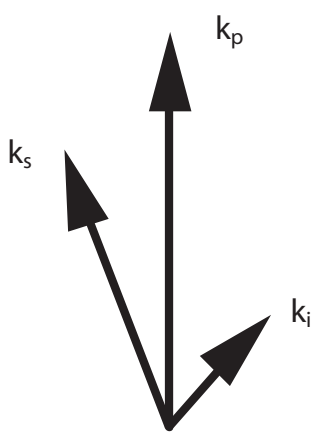

(c)

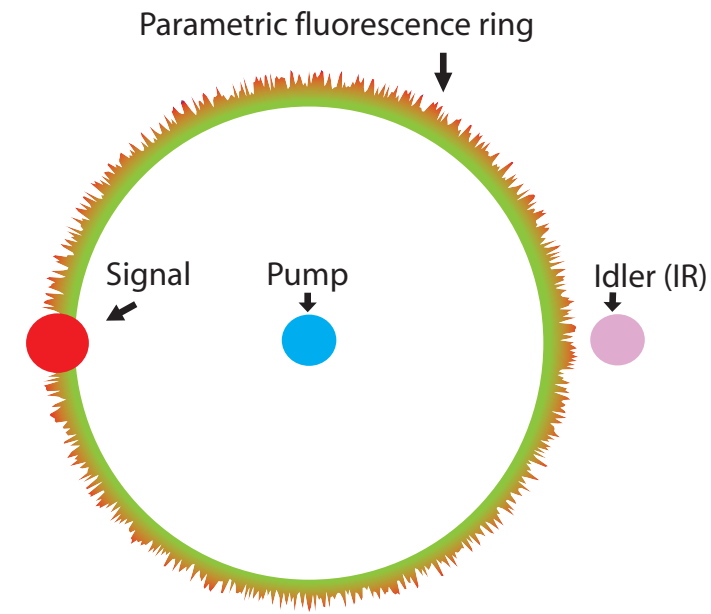

Figure 3.1: Diagrams of NOPA beam geometry. (a) shows the alignment of pump and seed into the BBO crystal from an overhead view. (b) gives the phasematching condition for amplification, and (c) shows a cartoon of NOPA signal and other beams immediately after the crystal. The pump, signal, and idler beams propagate in a plane parallel to the table.

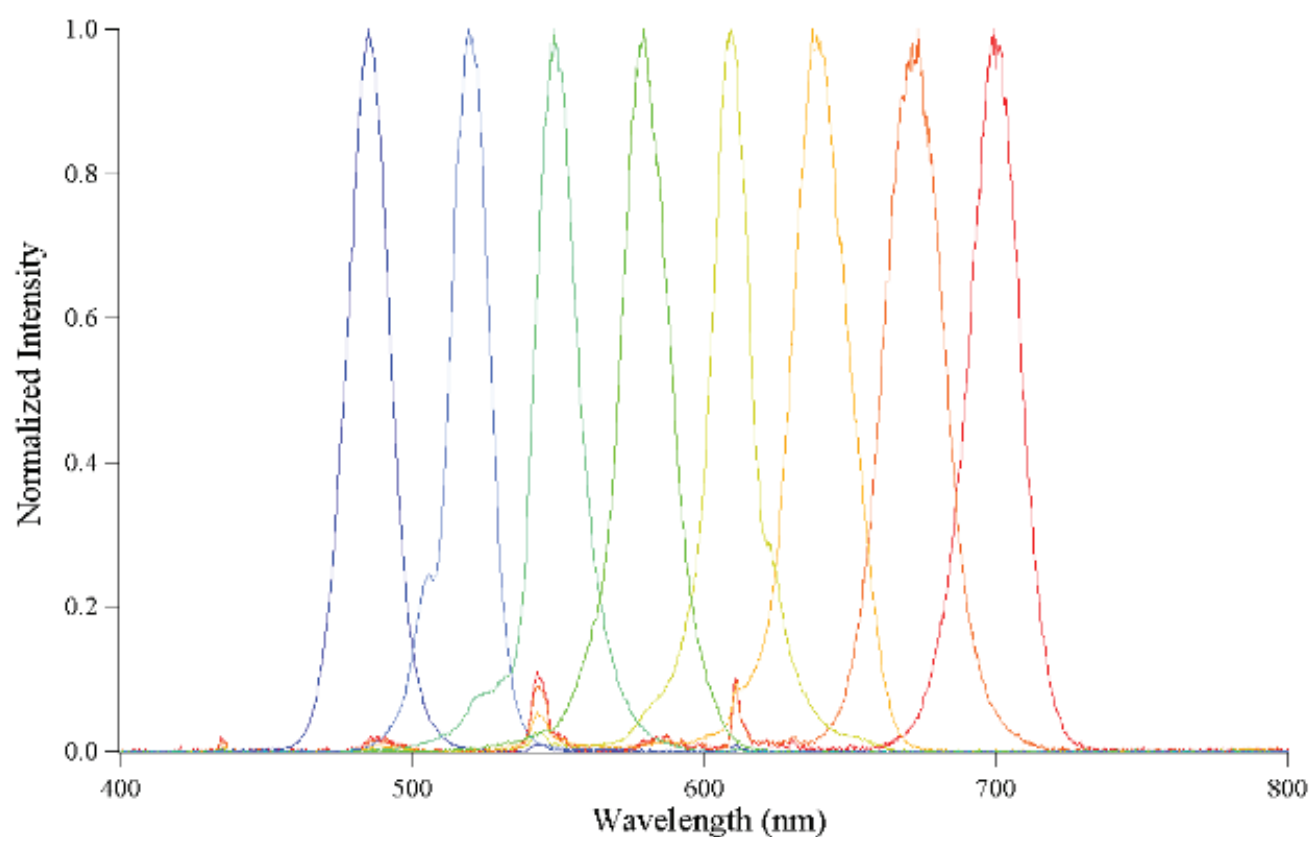

Figure 3.2: A series of experimentally obtained NOPA spectra, demonstrating the broad tunability of our apparatus. 


\subsection{Prism Compressor}

In order to optimally compress the pulses for the high temporal resolution required for many of our experiments, a setup for dispersion compensation is necessary. We compress our pulses with the aid of a prism compressor [4]. Pulses from the NOPA enter the first prism near the tip and are spectrally dispersed before propagating to a second identical prism. This collimates the beam in the Fourier plane, in which the horizontal spatial dimension defines a frequency axis. A folding mirror reflects the beam back through the prisms along a similar path but tilted slightly downward so that the beam can be picked off with a mirror after exiting the compressor.

The separation between the two prisms can be tuned to compensate for a given amount of dispersion in the setup, which is itself a function of the frequency of the pulse. This effect is based on a geometric effect and can be predicted analytically $[4,5]$. The prisms are mounted on micrometer stages that translate perpendicular to the rear face of the prism in order to make fine adjustments of separation and to add additional material dispersion when necessary without changing the beam pointing. For the PSII experiments, we chose SF-11 prisms which allow for smaller separations due to the increased frequency spreading from larger material dispersion. Fused silica prisms were used for other experiments.

The prism compressor can be used to pre-compensate for dispersion later along the beam path, as well as for dispersion on the beam before it enters the compressor. In our experiments, we precompensate the pulses so as to get them near the transform limit when they interact with the sample. It should be noted that a prism compressor is most useful for compensating second-order phase (linear chirp) but is not ideal for higher order phase terms. In applications where higher order phase compensation is desired, a grating compressor or deformable mirror should be employed in addition to the prism compressor. Pulse characteristics were measured with a ZAP-SPIDER setup [6] (see Appendix B). 


\subsection{Requirements for 2D Spectra}

In order to collect reliable data, there are several requirements which must be met. A 2D spectrum measures the complete complex electric field of the signal pulse through heterodyning techniques. In order to achieve this precision, the phase differences between the two pump pulses and between the probe and local oscillator pulses must be constant over the course of a measurement. For fully non-collinear techniques, this require phase stability on the order of $\lambda / 100$ over the course of several minutes.

Another important factor is the precise control and knowledge of the time delays, specifically the coherence time $t_{1}$. Timing errors can distort lineshapes by mixing different signal contributions improperly. The discussion of the two implementations of 2DES experiments below will address these issues in the context of each method.

In our lab, we have implemented two different but complementary experimental setups for collecting 2DES data. The first of these utilized a diffractive optic to create pulse pairs and a refractive delay for $t_{1}$, while the second setup uses a pulse shaper to create the $t_{1}$ delay and also to compensate the pump beam for dispersion. The pulse shaper setup utilizes a simpler pump-probe beam geometry, as opposed to the boxcar geometry in the diffractive optic setup.

\subsection{DES with a Diffractive Optic}

In the diffractive optic setup (Figure 3.3), the output of two individually tunable NOPAs are pre-compensated in separate prism compressors. The beams are focused with a slight vertical offset onto a transmissive diffractive optic. The diffractive optic is optimized for the \pm 1 diffraction orders at visible wavelengths, so that each beam gets split into two replicas with trajectories that diverge horizontally from each other. The four beams are then collimated with a parabolic mirror. The beams form 
a "boxcar" geometry, in which each beam lies on the corner of a square.

After the collimation, the beams are well separated as they propagate, allowing for different optics to be placed in each beam. In the most direct implementation, this spatial separation allows us to scan refractive time delays along individual beams and attenuate the amplitude of the local oscillator pulse. If desired, waveplates can be used to individually control the polarization of each pulse. The fact that the beams all hit the same reflective optics between the diffractive optic and the sample ensures the excellent passive phase stability on the order of $\lambda / 100$ necessary for this experiment [7].

The two beam copies from one of the NOPAs serve as the pump pulses for our four-wave mixing experiments, while the pulses from the other NOPA become the probe pulse and local oscillator for heterodyne detection, respectively. Each of the pump pulses passes through an adjustable refractive delay formed by a pair of glass wedges, one of which is on a motorized stage and can be translated relative to the other [8]. The effect of translating the wedge is that the thickness of the glass in the beam path changes in small increments, which effectively increases or decreases the time delay of that pulse relative to the others at the sample. A pair of wedges oriented anti-parallel to one another ensures that the beam path will not be diverted and also that the entire spatial profile of the beam will encounter the same thickness of glass. By placing a wedge pair in each pump beam instead of just one of them, we can separately scan the $t_{1}$ delay to recover rephasing or non-rephasing signal (depending on the pulse ordering) without changing the population time $\left(t_{2}\right)$. We place one wedge from each pair on an adjustable tilt mount to allow for fine adjustment of the wedge alignment which is crucial for minimizing beam pointing errors.

The phase of the signal pulse is dependent on the phases of the other pulses and 
follows the relationship [7]

$$
\Delta \Phi_{h e t}=\left(\Phi_{1}-\Phi_{2}\right)+\left(\Phi_{3}-\Phi_{l o}\right)+\Phi_{s}
$$

To ensure an accurate retrieval of the signal phase from the hererodyne detected phase, the relative phases between the pairs 1-2 and 3-lo must be very stable, on the order of $\lambda / 100$. In this implementation, the use of the diffractive optic provides passive phase stability. The pulses of each pair are produced by the same beam, and between the diffractive optic and the sample, the beams all hit the same set of mirrors, which mitigates the effect of mechanical vibrations on the phase.

The local oscillator pulse is attenuated with a neutral density filter of optical density 3.0. The probe pulse is transmitted through a $1 \mathrm{~mm}$ thick fused silica window to ensure that the local oscillator pulse will arrive in the spectrometer before the signal. The delay between probe and local oscillator pulses is measured daily with spectral interferometry [9] and is generally $\sim 1430 \mathrm{fs}$.

The beams hit a second parabolic mirror which focuses and converges them onto the sample. The phase-matching conditions $\mathbf{k}_{s}^{(R)}=-\mathbf{k}_{1}+\mathbf{k}_{2}+\mathbf{k}_{3}$ for the rephasing signal and $\mathbf{k}_{s}^{(N R)}=\mathbf{k}_{1}-\mathbf{k}_{2}+\mathbf{k}_{3}$ for the non-rephasing signal ensure that the rephasing(non-rephasing) signal will be collinear with the local oscillator for pulse orderings in which the pump beam $\mathbf{k}_{1}\left(\mathbf{k}_{2}\right)$ interacts with the sample first. To collect a $2 \mathrm{D}$ spectrum, we begin with the pump pulses temporally overlapped and keep pulse 2 stationary while continuously scanning pulse 1 backward in time by translating the one of the paired wedges in that beam so as to reduce the thickness of glass the pulse encounters, thus increasing the relative delay $t_{1}$. The signals collected will be Fourier transformed across $t_{1}$ to form a rephasing scan for a single $t_{2}$ delay. We repeat this procedure, scanning beam 2 while beam 1 is stationary, in order to obtain the nonrephasing data. The population time $t_{2}$ is set with a retroreflector on a motorized 
stage before the diffractive optic so as not to change the relative delays between the probe and local oscillator. The retroreflector stage and wedge delays are scanned by a computer, which controls a digital signal processor (DSP)(Motorola DSP56F807). The DSP scans the refractive delays continuously between user-defined start and stop values and outputs a TTL pulse while the motors are moving. The TTL pulse is sent to a digital logic "AND" gate, where it is evaluated with a $1 \mathrm{kHz}$ trigger from the regen to produce an output trigger. The DSP records the motor position corresponding to each laser pulse with the use of a high-precision digital counter signal.

The heterodyned signal pulses are dispersed in a spectrometer (Horiba Jobin-Yvon iHR320) and recorded on a self-cooling charge-collecting device (CCD) (Princeton Instruments Pixis 100B), where the exposure time is gated by the same trigger that controls the recording of motor positions. By hardware binning the signal over several lines of pixels, we are able to read out data in real-time at the $1 \mathrm{kHz}$ repetition rate of our laser, allowing for continuous scanning of the $t_{1}$ delay.

Calibration of the $t_{1}$ delay is performed by placing a $10 \mu \mathrm{m}$ pinhole in the sample position and blocking the probe and local oscillator beams. The pinhole scatters light from both pump beams, thus creating a spectral interferogram which is collected with the CCD. The wedges are scanned as in a normal experiment and motor positions are collected corresponding to each linear interferogram. By performing spectral interferometry [9], we retrieve a linear relationship of $t_{1}$ as a function of motor position for each motor, letting us know both the step size of $t_{1}$ and giving a reasonably accurate approximation of the position corresponding to $t_{1}=0$, the precise knowledge of which is necessary for proper phasing of spectra.

Because signal intensities are often too low to measure directly with high SNR and an intensity measurement gives no information of the phase, we instead heterodyne our signals with a local oscillator pulse, giving us a spectral interferogram (Figure 3.4). The measured trace then consists of a mixture of the local oscillator and signal 
(a)

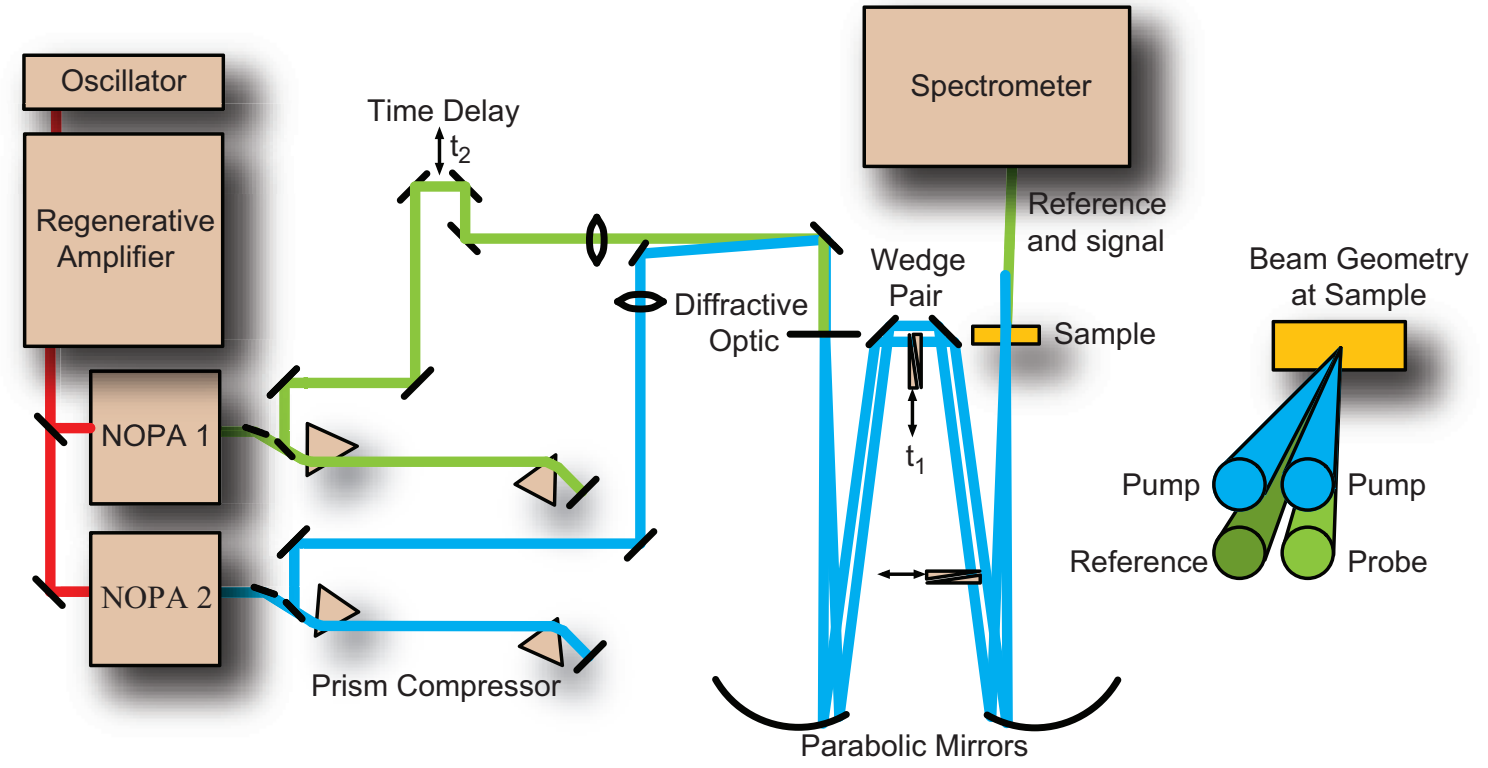

(b)

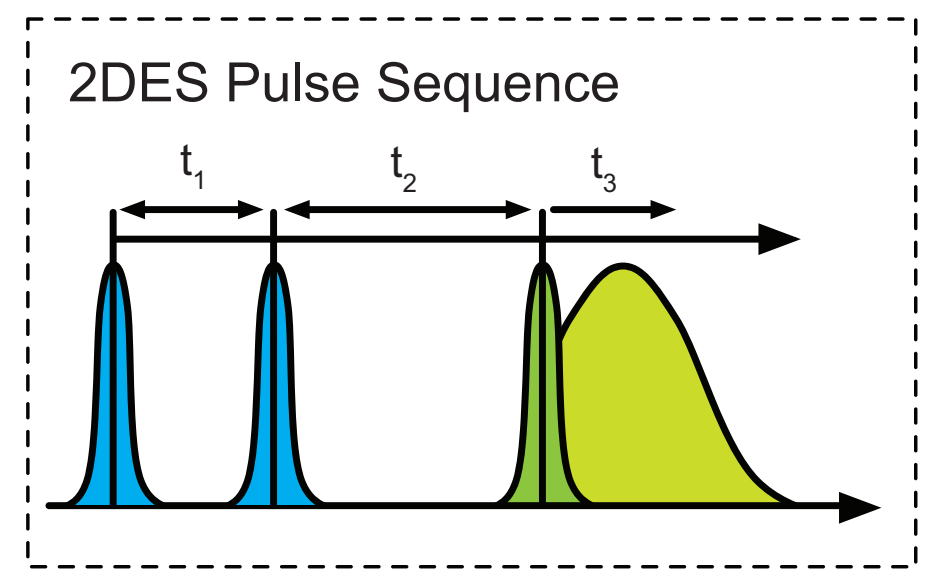

Figure 3.3: Experimental setup for 2DES with a diffractive optic and pulse sequence. (a) Diagram of the experimental setup for 2DES with a diffractive optic. The boxcar beam geometry is shown on the right. (b) The pulse sequence for $2 \mathrm{DES}$. 


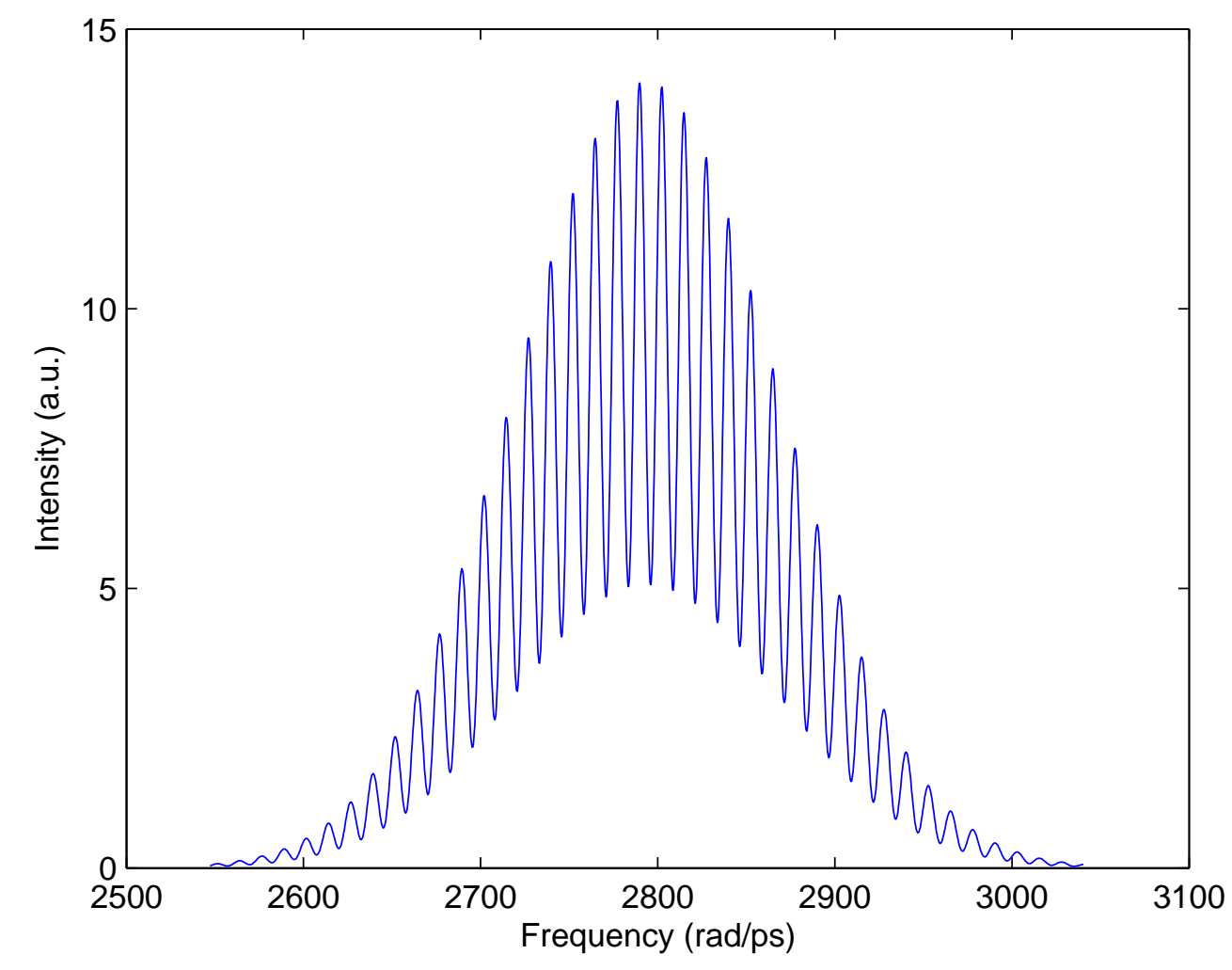

Figure 3.4: A simulated spectral interferogram. This shows the measured signal from equation 3.3 .

pulse fields given by

$$
\tilde{S}(\lambda)=\left|E_{s}(\lambda)\right|^{2}+\left|E_{l o}(\lambda)\right|^{2}+\tilde{E}_{s}(\lambda) \tilde{E}_{l o}^{*}(\lambda) e^{i \omega \tau}+\text { c.c. }
$$

where $\tilde{E}_{l o}$ is the complex electric field of the local oscillator, $\tilde{E}_{s}$ is the complex electric field of the signal, and $\tau$ is the time delay between the two heterodyned pulses.

During data analysis, the spectral interferogram, measured in evenly spaced wavelength, is Fourier transformed into a pseudotime axis [9]. Because of the large delay between the signal and local oscillator, the desired interference term is well separated from the DC term and is windowed with a super-Gaussian filter (see Figure 3.5). The filtered interference is then inverse Fourier transformed back into the wavelength domain, yielding a complex, zero-mean interference pattern (Figure 3.6). The phase 


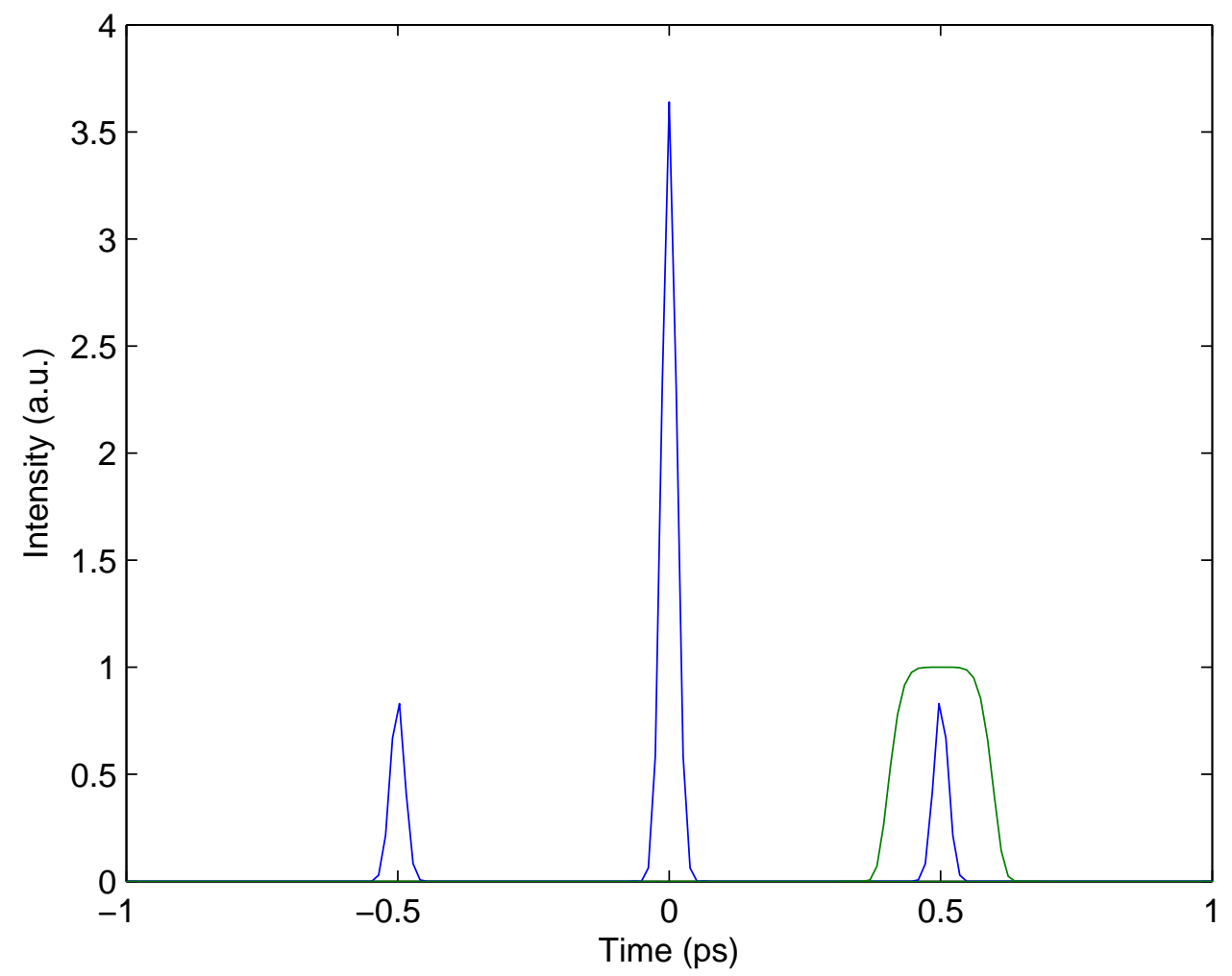

Figure 3.5: A time domain trace of the inverse Fourier transform of a spectral interferogram (blue curve), including a DC contribution as well as the two well-separated interference terms. The green curve shows the windowing of the interference term corresponding to the correct pulse ordering.

is extracted and unwrapped, and the linear term $\omega \tau$ is subtracted. The remaining phase is that of the signal alone and is reapplied to the amplitude term. The electric field term from the local oscillator, $E_{l o}$ is measured separately or taken from a time delay where no signal is expected and is divided out to yield a complex signal field

$$
\tilde{E}\left(\omega_{3}\right)=E\left(\omega_{3}\right) e^{i \phi_{s}\left(\omega_{3}\right)}
$$

After spectral interferometry is performed separately on the one-dimensional spectrum corresponding to each $t_{1}$ delay, the spectra are then represented together in the time-frequency domain as $\tilde{S}\left(t_{1}, \omega_{3}\right)$. A Fourier transform produces our final $2 \mathrm{D}$ spectrum: 


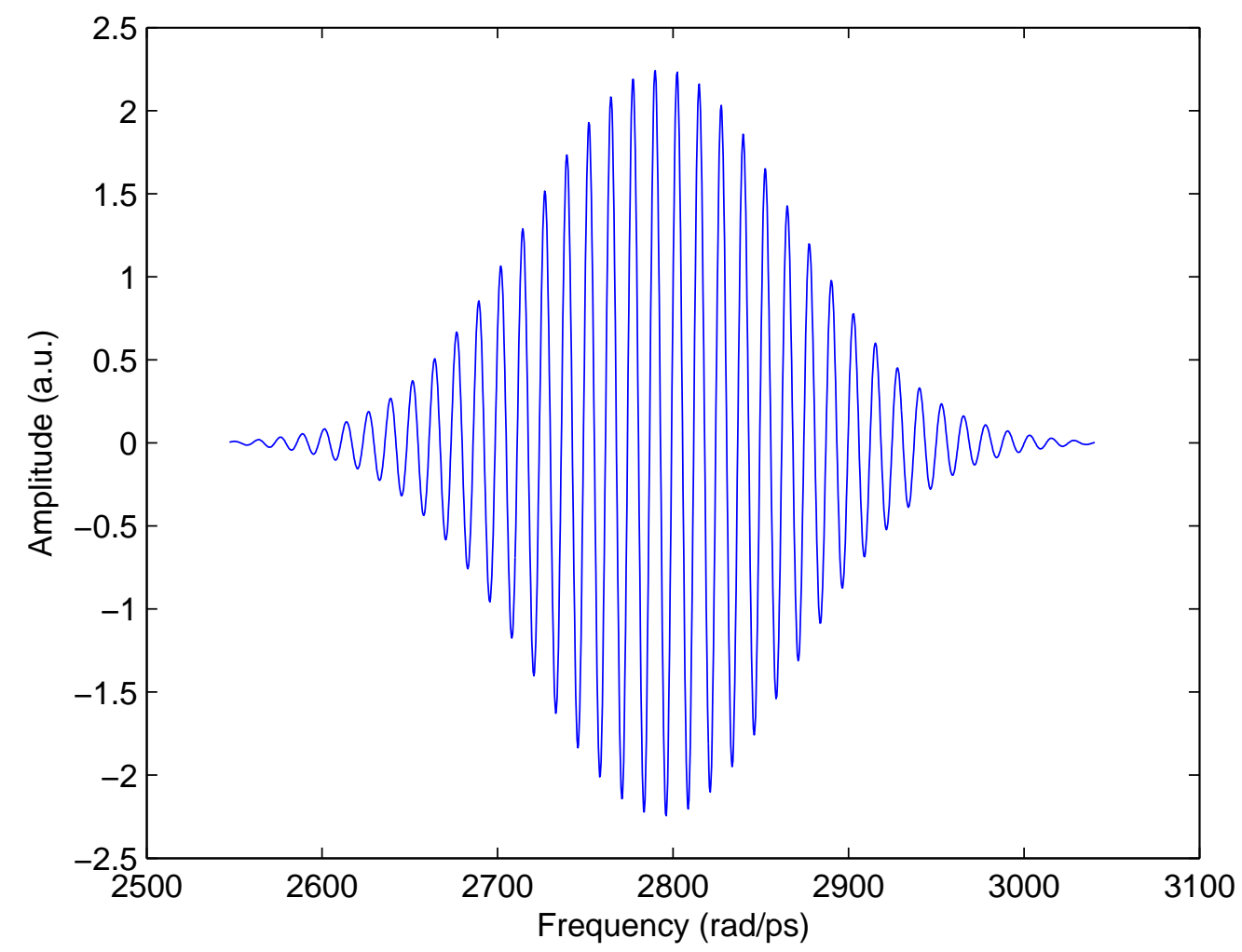

Figure 3.6: Real part of the zero-mean complex signal interference term after windowing in the time domain. 


$$
\tilde{S}\left(\omega_{1}, \omega_{3}\right)=\int \tilde{S}\left(t_{1}, \omega_{3}\right) e^{i \omega_{1} t_{1}} d t_{1}
$$

In this way, we obtain separate rephasing and non-rephasing spectra for a given $t_{2}$ delay. These can be analyzed individually in order to accentuate certain features or dynamics $[10,11]$. These two separate signal contributions are not usually preferred, though, as they each contain phase twists which broaden the lineshapes [12]. For most applications, researchers prefer to examine an absorptive spectrum, which boasts the highest frequency resolution and therefore the sharpest spectral features.

In order to obtain an absorptive lineshape, however, the rephasing and nonrephasing spectra must be added together with precise relative timing of the two signals. Causality requires that signal be present for both time orderings of the pulses giving rise to the coherence during $t_{1}$. Were there no population dynamics to be concerned with, the whole absorptive spectrum could be measured by scanning $t_{1}$ with a single pulse for positive and negative times. However, doing this in practice can distort the spectra by mixing different $t_{2}$ values in a single spectrum. Instead, we measure both the rephasing and non-rephasing signals, which corresponds to a time inversion of the two pump pulses and therefore includes both positive and negative $t_{1}$ contributions $[13,14]$.

The absorptive and dispersive parts of the spectrum are then given as

$$
\begin{aligned}
& S_{a b s}=\operatorname{Re}\left[\left(S^{R}+S^{N R}\right) e^{i \phi}\right] \\
& S_{d i s}=\operatorname{Im}\left[\left(S^{R}+S^{N R}\right) e^{i \phi}\right]
\end{aligned}
$$

It is important to note that spectral interferometry gives only the relative phase difference between the signal and local oscillator $\Delta \Phi_{\text {measured }}=\Phi_{s}-\Phi_{l o}$. The phase term $\phi$ from Equations 3.6 and 3.7 that is required to phase the spectra into absorptive and dispersive components is called the "global phase". Because the global phase 
is unknown and difficult to measure, the phasing is usually done by sampling phase values and comparing the resulting phased spectra to a separate transient absorption experiment, using the projection slice theorem $[14,15]$. After a test phase is applied, the $2 \mathrm{D}$ spectrum is integrated along $\omega_{1}$, resulting in a $1 \mathrm{D}$ signal which is identical to a broadband pump / broadband probe transient absorptions spectrum. This TA spectrum is easily obtained in our setup by blocking the pump beam $\mathbf{k}_{2}$ and the reference pulse and measuring the transient absorption signal between $\mathbf{k}_{2}$ and $\mathbf{k}_{3}$. No realignment before the sample is necessary, and this data can be taken immediately after a corresponding 2D spectrum to minimize sample degradation. The TA spectrum and integrated 2D spectrum are both normalized to unity, and a least squares fit is performed to minimize the difference between them for a given $t_{2}$ delay, using the global phase as a fitting parameter.

\subsection{DES with a Pulse Shaper}

While there are excellent reasons to use a fully non-collinear 2D setup, it has been pointed out that some of the technical challenges could be eliminated by performing 2DES in a pump-probe geometry [14]. In the pump-probe geometry, best known for its use in transient absorption experiments, the pump beams are collinear $\left(\mathbf{k}_{1}=\mathbf{k}_{2}\right)$, so the phase-matching conditions $\mathbf{k}_{s}^{(R)}=-\mathbf{k}_{1}+\mathbf{k}_{2}+\mathbf{k}_{3}$ and $\mathbf{k}_{s}^{(N R)}=\mathbf{k}_{1}-\mathbf{k}_{2}+\mathbf{k}_{3}$ simplify such that the rephasing and non-rephasing signals are collected simultaneously along the direction of the probe pulse. While this approach was identified long before the first 2DES experiments were performed [16], it was only recently implemented in the IR by the Zanni group using first a germanium acousto-optic pulse shaper [17] and later a Mach-Zehnder interferometer [18]. Shortly thereafter, our group published the first study using a pulse shaper to perform 2DES in the pump-probe geometry [19].

In our implementation, the beam from one NOPA enters an acousto-optic pulse shaper (Fastlite Dazzler) [20], where an RF pulse propagated through a crystal can 
shape the pulse with an arbitrary amplitude and phase within the spectral envelope of the input pulse. By generating an interferogram in the frequency domain, the pulse shaper effectively creates a pair of collinear pump pulses with a well characterized, tunable time delay of $0-400 \mathrm{fs}$. The modulation applied to produce the pulse pair is of the form $|E(\omega)|\left(1+\exp \left[i\left(\omega t_{1}+\phi_{12}\right)\right]\right)$, where $E(\omega)$ is the spectral amplitude of the pulse and $\phi_{12}$ is the relative carrier wave phase shift. Unlike spectral modulations produced by an interferometer, the pulse shaper allows the introduction of an arbitrary carrier wave phase shift that can be used for phase cycling schemes analogous to those used in NMR. Because the Dazzler can control the phase of the pulses, it is used to compensate dispersion up to the fourth order, so no prism compressor is required for this beam.

The probe pulse is taken from the other NOPA, though in later implementations a continuum pulse was used (see Section 3.6). A delay stage in the probe path controls the population time $t_{2}$. Because the pump pulses are collinear, the phase-matching condition $\mathbf{k}_{s}= \pm \mathbf{k}_{1} \mp \mathbf{k}_{2}+\mathbf{k}_{3}$ ensures that the rephasing and non-rephasing signals will both be collinear with the probe and can be collected simultaneously for all values of $t_{1}$. Therefore, the probe pulse is used as the local oscillator in a pump-probe beam geometry, greatly simplifying alignment. Because the delay $t_{1}$ between pump pulses is precisely controlled and the local oscillator occurs at exactly $t_{3}=0$, there is virtually no timing error in this experiment.

Because there is no time delay between the probe pulse and local oscillator, the measured signal has no interference fringes, so the desired signal is not separable from the larger contribution of the local oscillator intensity $\left|E_{l o}\right|^{2}$ in the time domain. Instead, we must subtract out the contribution of the probe intensity in the spectrum by recording a separate spectrum in which the signal is not present or has an opposite phase, while the probe intensity remains constant.

The most direct implementation of this is to use the pulse shaper to alternate 


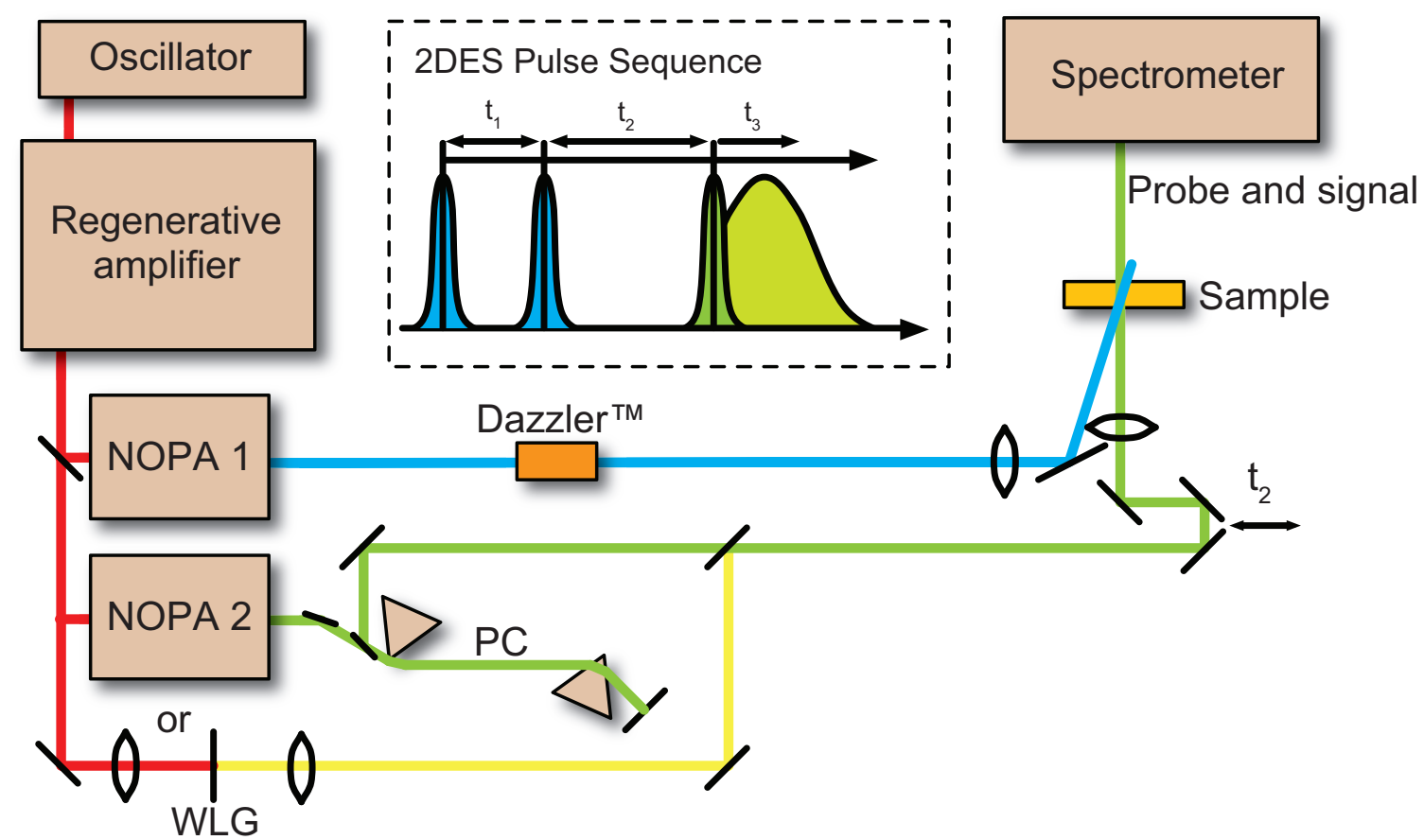

Figure 3.7: Diagram of the experimental setup for 2DES with a pulse shaper. Alternate beams can be chosen using a NOPA and prism compressor (PC) or white light generation (WLG). The inset shows the pulse sequence used in 2DES experiments. 
between on and off states, effectively chopping the pump beams. In this case, only half of the spectra taken have data, so the effective duty cycle is $50 \%$. To reach a full duty cycle, the phase difference between the pump pulses, $\Delta \phi_{12}$ can be cycled between 0 and $\pi$. This changes the phase of the signal and gives an additive effect when measured traces from the two phase schemes are subtracted from one another. Other phase cycling schemes can be utilized to remove scatter and will be discussed in Section 3.6.

For each $t_{1}$ value, we average over many spectra (typically 100-200) to improve the SNR of the data. Difference spectra are taken between successive frames on our CCD readout corresponding to chopped/unchopped pumps or different phases, and after subtraction we are left with a map of signal in the time-frequency domain: $S\left(t_{1}, t_{2}, \omega_{3}\right)$. The signal is still real at this point. To obtain a complex signal field and separate out the absorptive (real) and dispersive (imaginary) contributions, we first take the Fourier transform along $t_{1}$ and select the real part of the signal before inverse transforming back. The signal is then inverse Fourier transformed along the other axis, $\omega_{3}$. Causality is enforced by windowing out all data corresponding to negative $t_{3}$ delays with a Heaviside function $\Theta\left(t_{3}\right)$ [21]. This simply means that we disallow any signal that appears to arrive before the probe pulse. The data is then Fourier transformed along $t_{3}$ and then $t_{1}$ to produce the final complex spectrum: $\tilde{S}\left(\omega_{1}, t_{2}, \omega_{3}\right)$.

\section{Separation into Rephasing and Non-rephasing signals}

One advantage of 2DES in the pump-probe geometry is that the rephasing and non-rephasing signals are collinear and collected at the same time, so no phasing of the spectrum is necessary, and there is no ambiguity regarding the calibration of $t_{1}=0$. It is sometimes helpful, however, to be able to look at the rephasing and non-rephasing contributions to the spectrum separately. De Boeij et.al. suggested a method for recovering the rephasing and non-rephasing components of frequency- 
resolved pump-probe signals $[22,23]$, which can easily be applied to 2DES in the pump-probe geometry. These data can be obtained by recording spectra in which the relative phase between pump pulses is $\Delta \phi_{12}=0$ and $\Delta \phi_{12}=\frac{\pi}{2}$, respectively. These spectra have the following relationships to the rephasing and non-rephasing spectra $[22,23]:$

$$
\begin{aligned}
S_{0}\left(t_{1}, t_{2}, t_{3}\right) & \propto R^{(R)}\left(t_{1}, t_{2}, t_{3}\right)+R^{(N R)}\left(t_{1}, t_{2}, t_{3}\right) \\
S_{\pi / 2}\left(t_{1}, t_{2}, t_{3}\right) & \propto-i R^{(R)}\left(t_{1}, t_{2}, t_{3}\right)+i R^{(N R)}\left(t_{1}, t_{2}, t_{3}\right)
\end{aligned}
$$

Combining these gives the separated signals

$$
\begin{aligned}
& S_{0}\left(t_{1}, t_{2}, t_{3}\right)+i S_{\pi / 2}\left(t_{1}, t_{2}, t_{3}\right) \\
& S_{0}\left(t_{1}, t_{2}, t_{3}\right)-i S_{\pi / 2}\left(t_{1}, t_{2}, t_{3}\right) \propto R^{(R)}\left(t_{1}, t_{2}, t_{3}\right) \\
& (N R)\left(t_{1}, t_{2}, t_{3}\right)
\end{aligned}
$$

This separation of rephasing and non-rephasing signals in the pump-probe geometry is shown for data from the laser dye LDS750 in acetonitrile in Figure 3.8

\section{Scatter Subtraction}

One of the difficulties in any implementation of 2DES is in removing contributions to the measured signal due to scatter and interference terms from different beams, as well as signals from other, unwanted nonlinear processes in the sample. This is particularly important for biological samples that sometimes form aggregates. Because the scatter is often nearly collinear with the signal pulse, spatial filtering provides only limited success. Zanni and coworkers presented an excellent phase cycling scheme for removing contributions due to scatter between the pump and probe pulses, as well as transient absorption signals due to multiple interactions of the sample with a single pump pulse [24]. This method does not, however, remove the interferometric scatter 

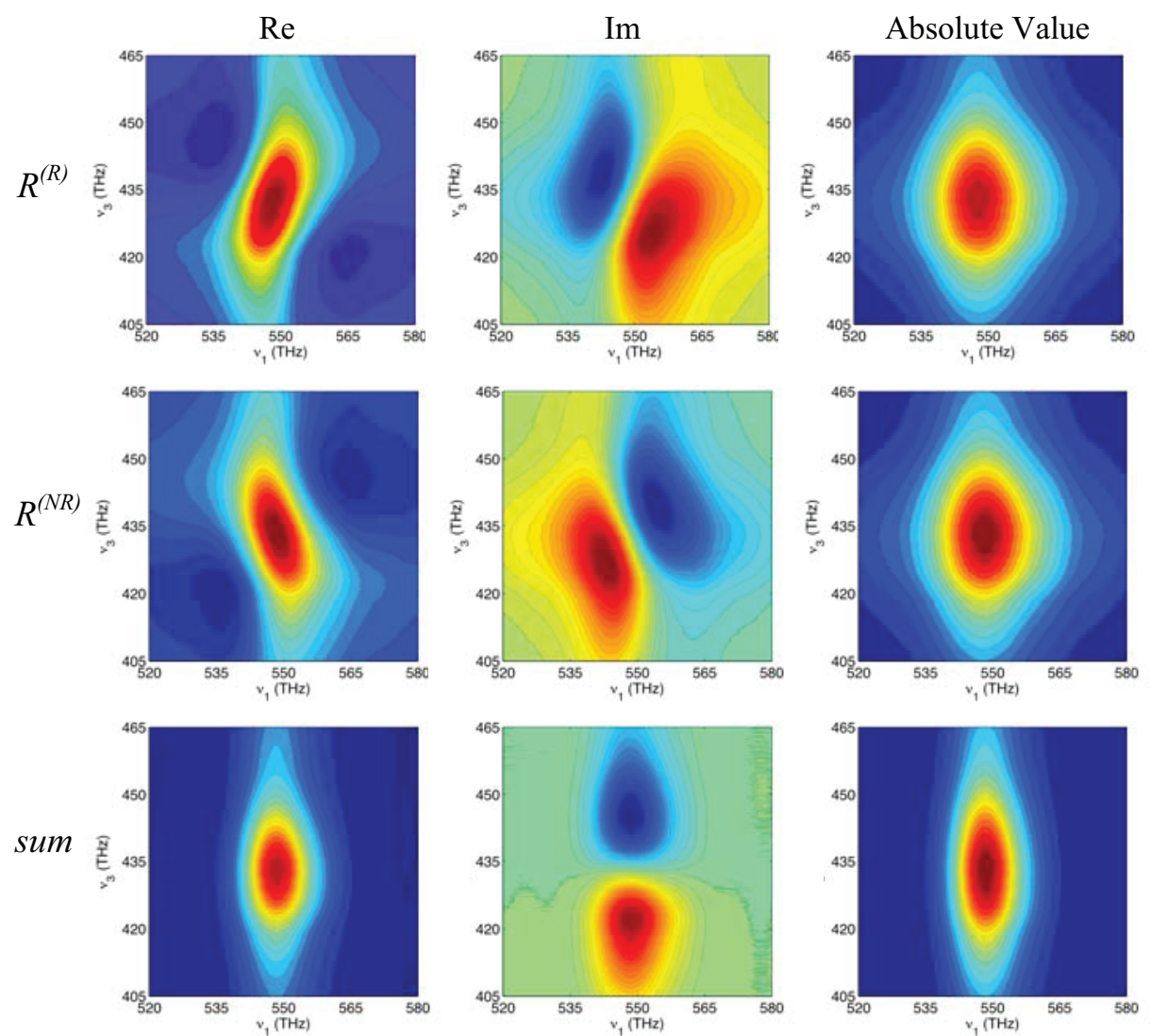

Figure 3.8: Separation of rephasing and non-rephasing contributions for LDS750 in acetonitrile at $t_{2}=500 \mathrm{fs}$. The columns contain real, imaginary and absolute value spectra as indicated. Top row: components of the rephasing signal. Middle row: components of the non-rephasing signal. Bottom row: addition of the rephasing and non-rephasing signals. 

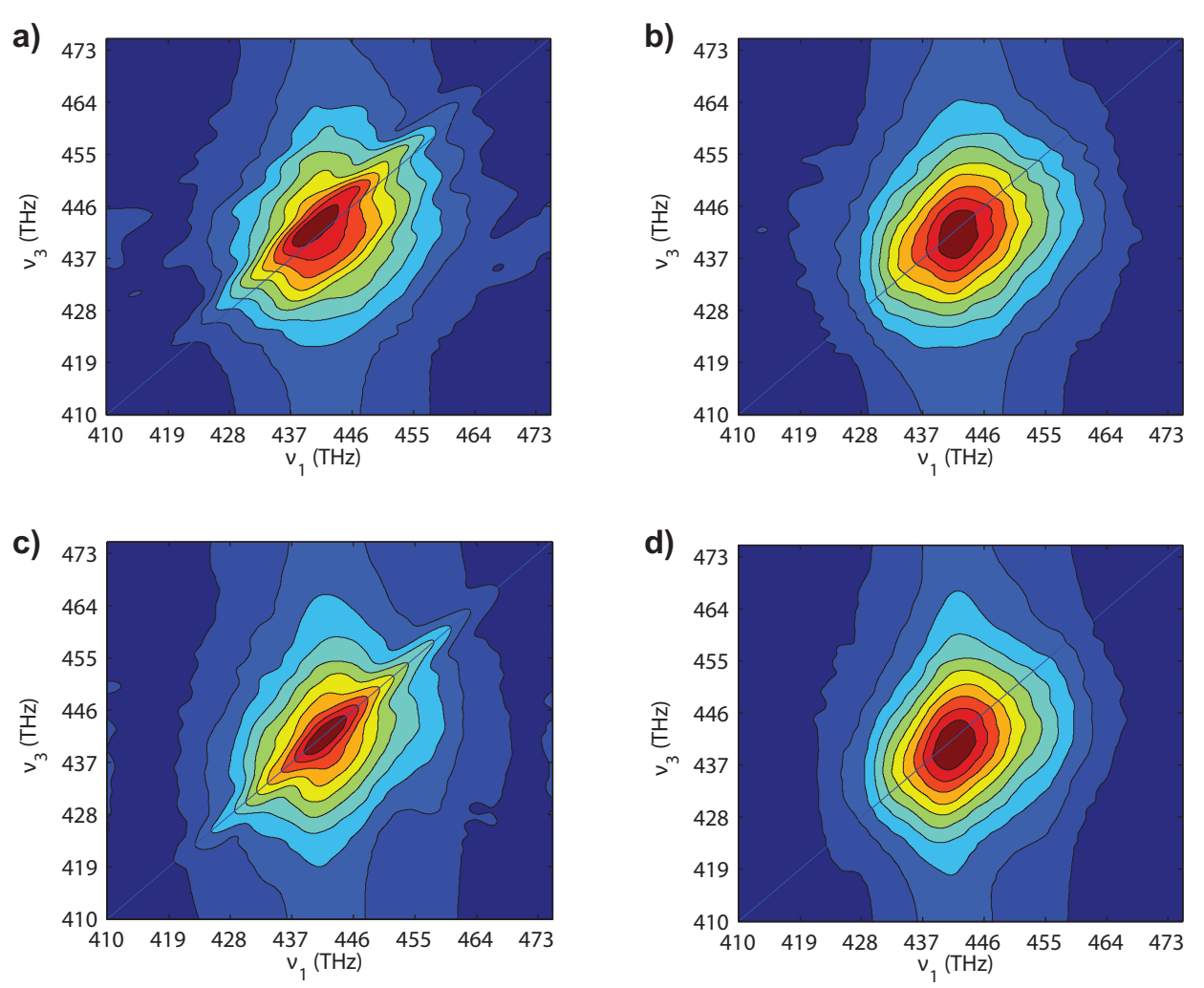

Figure 3.9: 2D spectra of rhodamine 800 in ethanol, showing different scatter subtraction schemes. a) Chopping only the pumps; no scatter signals are removed. b) Chopping both the pumps and the probe; removes pumppump scatter. c) Four-phase cycling scheme; removes all unwanted contributions except for pump-pump scatter. d) Four-phase cycling with probe chopping; only the desired 2D signal remains.

between the two pump beams which frequently shows up along the diagonal of our $2 \mathrm{D}$ spectra. We have implemented these methods in our lab and added a chopper in the probe pulse. Taking difference spectra between signals with a chopped vs. unchopped probe pulse allow us to isolate and subtract a strong scatter signal from the interference between the two pump beams, which shows up as a narrow peak along the diagonal. Results of this scatter subtraction are shown in Figure 3.9. 


\section{Continuum Probe}

Many sample systems of interest such as the natural light harvesting systems contain transitions across a broad range of frequencies. In these applications, it is often desirable to obtain information over several well-separated spectral components at once. The use of a continuum probe pulse permits this [25] and is readily used in the pump-probe geometry.

The continuum probe pulse is created by focusing a small fraction of the $800 \mathrm{~nm}$ regenerative amplifier output into a $2 \mathrm{~mm}$ thick sapphire window with a $200 \mathrm{~mm}$ lens. The continuum is collimated using a $50 \mathrm{~mm}$ lens and can be compressed further or used as-is with a sizeable linear chirp. The spectrum of our continuum pulse is shown in Figure 3.10.

\subsection{Demonstration Experiments}

While this thesis culminates in the successful 2DES investigation of the D1D2cyt.b559 reaction center complex, a great deal of my time in the Ogilvie group was spent building both of our 2DES implementations from scratch, debugging our system, developing more advanced data analysis methods and coding the software, and finding my way through the unforeseen challenges of a method where the strengths and difficulties are still not fully understood by the experts.

In this section, I discuss briefly two studies we performed in the course of development which highlight some of our specific contributions to the field of two-dimensional electronic spectroscopy from a technical standpoint and demonstrate the successful operation of both diffractive optic and pulse shaper 2D experiments. The first of these utilized the diffractive optic implementation to measure the Förster energy transfer between a pair of dyes connected by a rigid bridge of DNA at a known separation (Figure 3.11). This experiment measured a cross peak far from the diagonal and was 


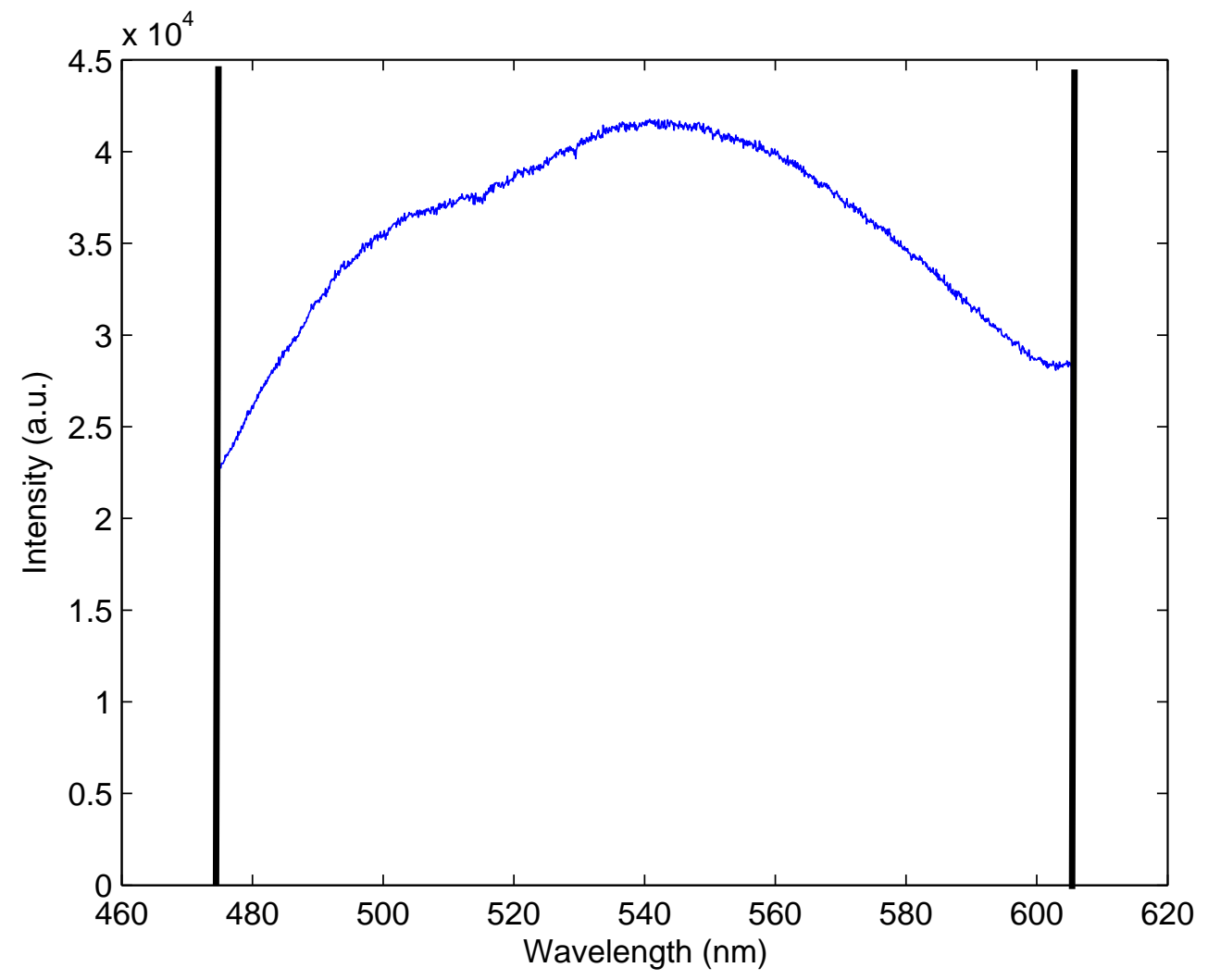

Figure 3.10: A spectrum of the continuum pulse produced with a Sapphire window. The black bars represent the spectral range of our spectrometer when centered at $546 \mathrm{~nm}$, which covers approximately the pulse FWHM at $130 \mathrm{~nm}$.

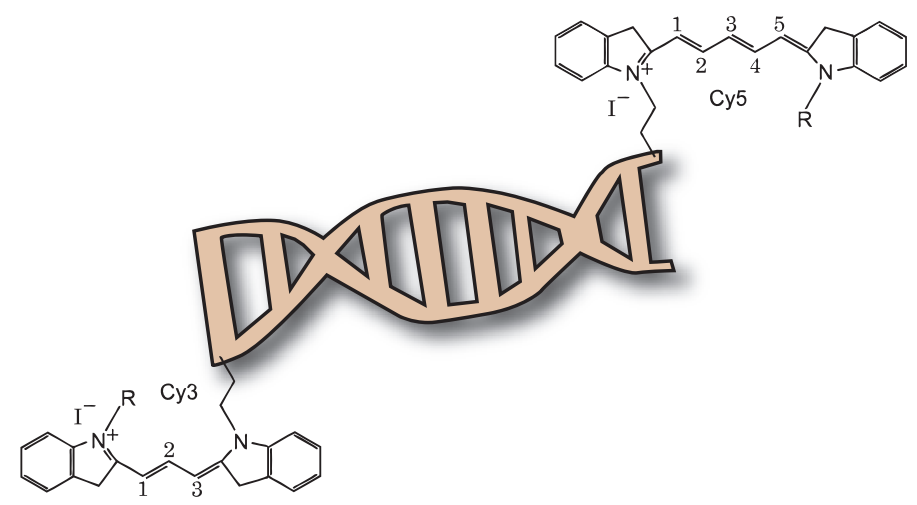

Figure 3.11: Cartoon structure of a DNA-bound FRET pair. 
the first two-color 2DES (2C2DES) experiment [26]. The second experiment used 2DES in the pump-probe geometry in conjunction with a continuum probe pulse to investigate vibrational wavepacket dynamics in a laser dye. This experiment was the first 2DES experiment performed with a continuum probe and as such was able to observe vibronic cross peaks in the dye spectrum [25].

\section{Color 2DES: Energy Transfer in a DNA Construct}

To demonstrate 2C2DES with the DO setup we chose to examine a simple sample in which absorption and emission wavelength were well separated. We note that our later development of $2 \mathrm{DES}$ with a continuum probe pulse would have worked as well for this experiment. The sample we chose for this experiment was a DNA construct consisting of donor and acceptor chromophores covalently bound to the 3' and 5' end of a short piece of DNA. This provides a fluorescence resonance energy transfer (FRET) pair with a rigid, well-defined separation that can be controlled by selecting an appropriate number of DNA base pairs. The absorption and fluorescence spectra of our dyes are shown in Figure 3.12 along with arrows indicating the frequencies of our pump and probe spectra.

Figure 3.13 shows the 2D spectrum of our DNA construct sample, consisting of an 8 base-pair piece of DNA labeled with donor (Cy3) and acceptor $(\mathrm{Cy} 5)$ dyes at either end (purchased from IDT technologies). Here we excited the donor (500 $\mathrm{nm})$ and probed the acceptor emission $(675 \mathrm{~nm})$ at $t_{2}=71 \mathrm{ps}$. The cross peak present in the $2 \mathrm{D}$ spectrum is indicative of energy transfer during $t_{2}$. We are able to properly "phase" our data by fitting the projection of the real part of our 2D spectrum onto the $\nu_{3}$ axis to a separately measured pump-probe signal, enabling us to properly separate absorptive and dispersive contributions to the $2 \mathrm{D}$ spectrum. Since the pump pulses may directly excite the acceptor, we performed an additional experiment to provide further evidence that the cross peak is due to energy transfer 


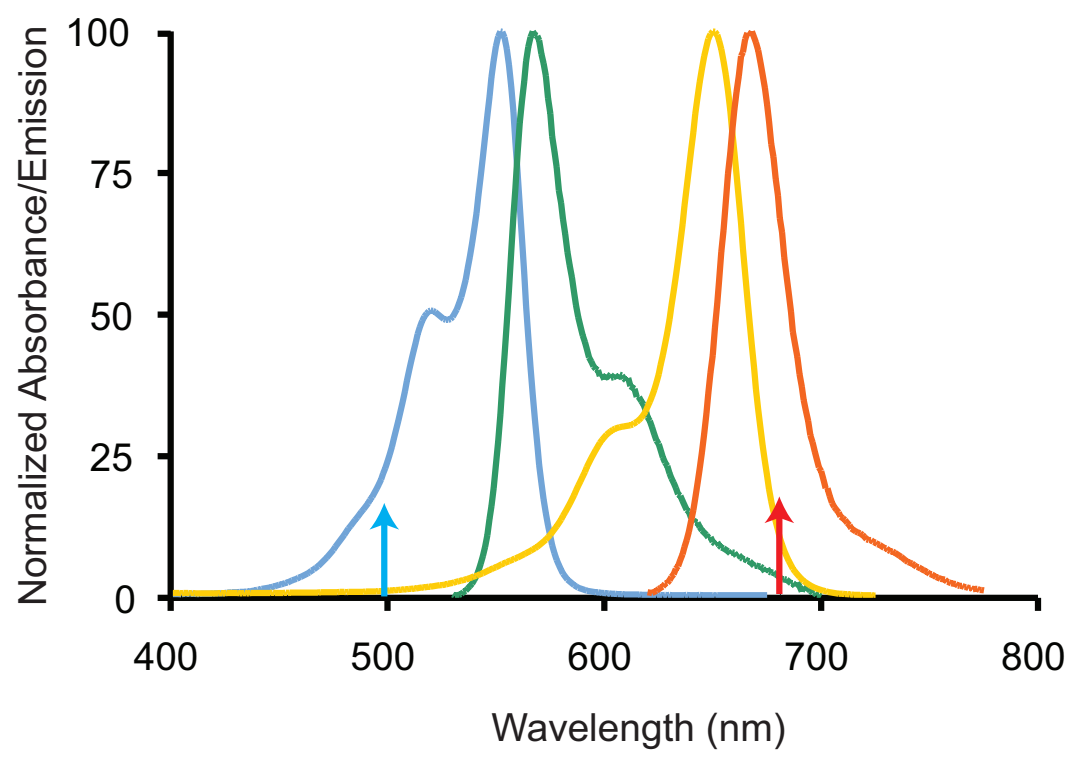

Figure 3.12: Absorbance (blue curve) and fluorescence (green curve) spectra of Cy3 and Cy5 (yellow and orange curves) from our duplexed DNA construct. With excitation at $500 \mathrm{~nm}$, direct excitation of the $\mathrm{Cy} 5$ is minimized and the large fluorescence signal is indicative of FRET. Assuming quantum yields of 0.04 for $\mathrm{Cy} 3$ and 0.27 for $\mathrm{Cy} 5$, this gives an estimated FRET efficiency of 25 percent. 

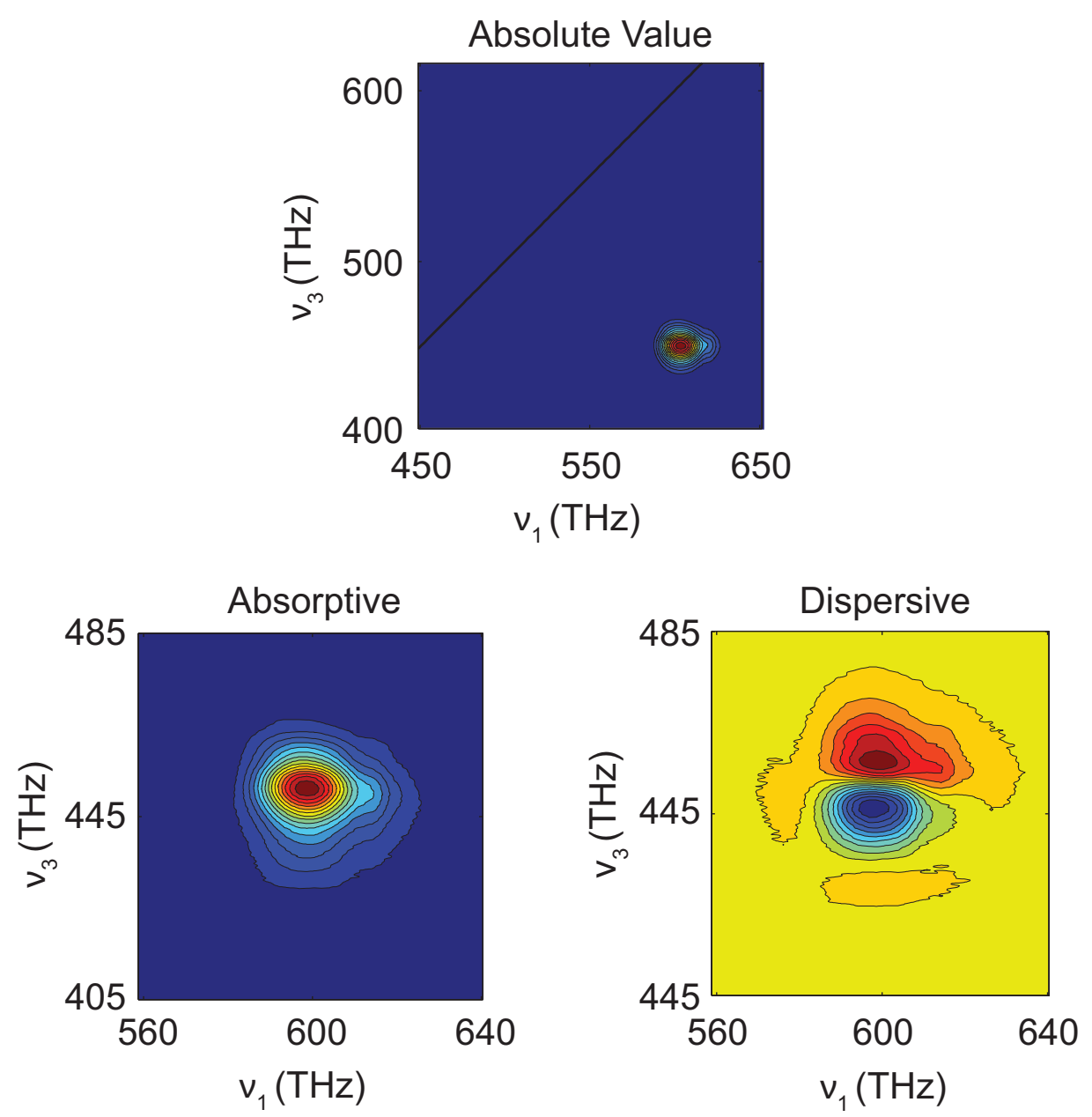

Figure 3.13: $2 \mathrm{D}$ spectra of the DNA at $t_{2}=71$ ps showing the cross-peak indicative of energy transfer.

and not emission from the directly excited acceptor. By pumping and probing the donor with and without the acceptor attached, we were able to compare the decay of the peak of the absolute spectrum. Figure 3.14 shows that the decay in the presence of the acceptor is significantly faster than without the acceptor, indicating energy transfer to the acceptor. 
(a)

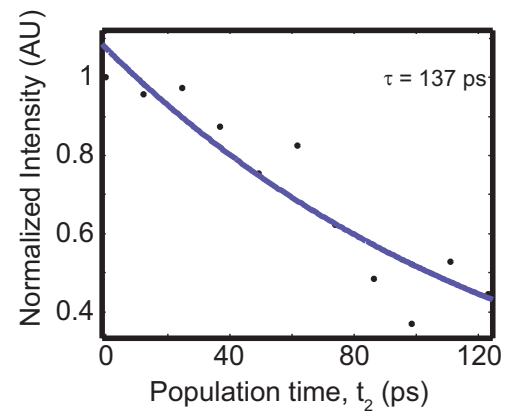

(b)

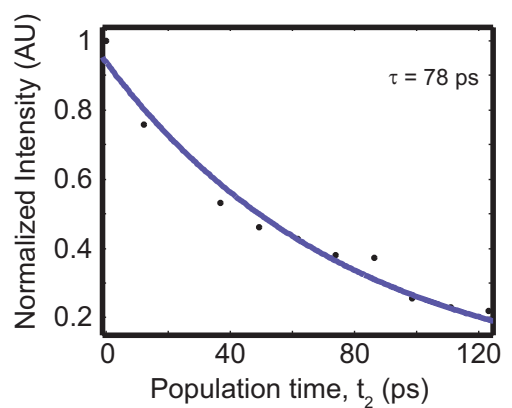

Figure 3.14: Comparison of decay rates for Cy3 in the presence and absence of Cy5. (a) The decay of the peak of the absolute value spectrum for DNA-bound Cy3.(b) When duplexed to a strand containing Cy5, the decay becomes faster, indicative of energy transfer.

\section{DES with a Pulse-Shaper: Continuum Probe Studies of Vibrational Wavepacket Dynamics in PERY}

To test the method of 2DES in a pump-probe geometry with a continuum probe, experiments were performed on the laser dye $N, N^{\prime}$-bis (2,6-dimethylphenyl) perylene3,4,9,10-tetracarboxylicdiimide (PERY), shown in Figure 3.15, dissolved in dimethyl sulfoxide (DMSO). This dye has been studied extensively by other groups using photon echo, transient grating, and one-color 2D spectroscopy [27, 28]. A prominent feature of PERY is the progression of a high-frequency vibrational mode $\left(1410 \mathrm{~cm}^{-1}\right)$ that modulates the absorption and emission spectra. Figure 3.16 shows the linear absorption of PERY as well as the spectra of our pump and probe pulses.

Results of the frequency-resolved pump-continuum probe experiments are shown in Figure 3.17(a). In addition to the signal from the dye molecule, signal from the nonresonant interaction of the pulses with the glass and solvent in the sample cell is also present. This nonresonant signal reaches a maximum during pulse overlap and does not influence the data at the relatively long $t_{2}$ times considered here. Since the probe pulse is chirped, the nonresonant signal peaks earlier at shorter wavelengths, providing a convenient way to quantify the chirp. In the pump-probe data we see a 


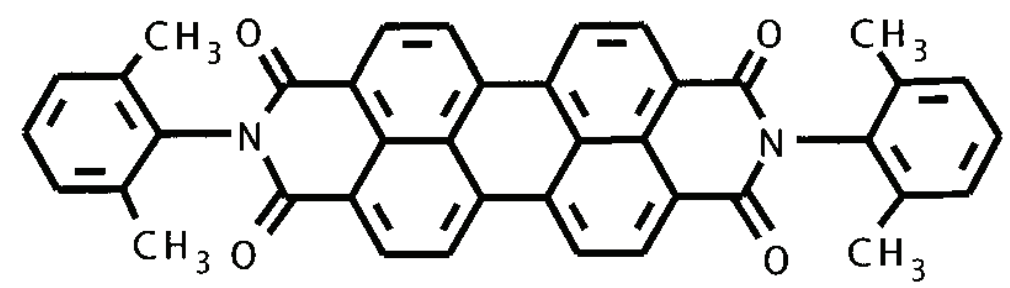

Figure 3.15: Chemical structure of PERY. This is adapted from [27].

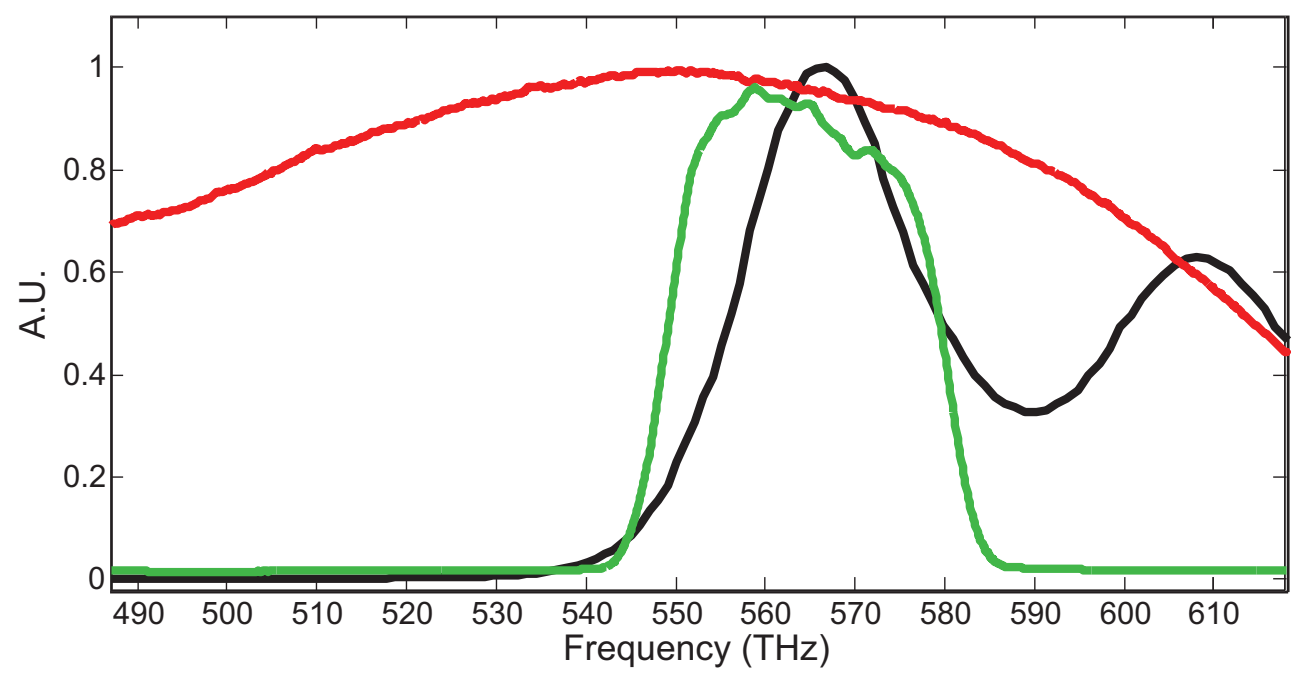

Figure 3.16: Absorption spectrum (black curve) of PERY showing the pump (green curve) and continuum (red curve) spectra. 
strong band centered at $\sim 560 \mathrm{THz}$ with a weaker band at $\sim 520 \mathrm{THz}$ and a very weak band at $605 \mathrm{THz}$. In Figure 3.17 we plot slices of the spectrally resolved signal at $518 \mathrm{THz}$ and $560 \mathrm{THz}$ without chirp correction. Both pump-probe traces show strong oscillations with a period of $\sim 240 \mathrm{fs}$, in good agreement with the previously observed intramolecular mode at $139 \mathrm{~cm}^{-1}$ [27]. The signal at $560 \mathrm{THz}$ can be assigned to a bleach of the ground state, whereas the $518 \mathrm{THz}$ signal is from excited state emission. When a chirp correction [29] is applied to the data, we find that the bleach and emission signals are approximately in phase.

Figure 3.18 shows the $2 \mathrm{D}$ spectra taken at different $t_{2}$ values indicated by the arrows in Figure 3.17(b), corresponding to maximum and minimum values of the 560 $\mathrm{THz}$ pump-probe signal. The horizontal axis is the excitation frequency obtained by Fourier transformation with respect to $t_{1}$, whereas the vertical axis represents the detection frequency obtained by spectrally resolving the signal. The dynamical evolution of the system can be followed by taking $2 \mathrm{D}$ spectra as a function of $t_{2}$. All data presented here are for $t_{2}$ times great enough to avoid effects of pulse overlap. There are three distinct peaks present in the 2D spectra located at approximately $\nu_{3}=605 \mathrm{THz}, \nu_{3}=560 \mathrm{THz}$, and $\nu_{3}=520 \mathrm{THz}$. For the central peak, initial excitation at the peak in the absorption (566 THz) leads to detection at $560 \mathrm{THz}$, which is a point part way between the absorption maximum and the first peak in the emission spectrum. This central peak has contributions from both GSB and Stokesshifted ESE, leading to a slight shift of the peak below the diagonal. Theory and model calculations to describe the effect of vibrations and relaxation on 2D electronic spectra have been previously reported [14, 30], although experiments have looked at systems where dephasing obscures closely spaced vibronic cross peaks $[8,28]$. In PERY, the large separation between the $0 \longrightarrow 0$ and $0 \longrightarrow 1$ transitions of the vibronic progression of the $1410 \mathrm{~cm}^{-1}$ mode (see Figure 3.16(a)), combined with the broad bandwidth of the continuum probe, permits the observation of vibronic cross peaks 


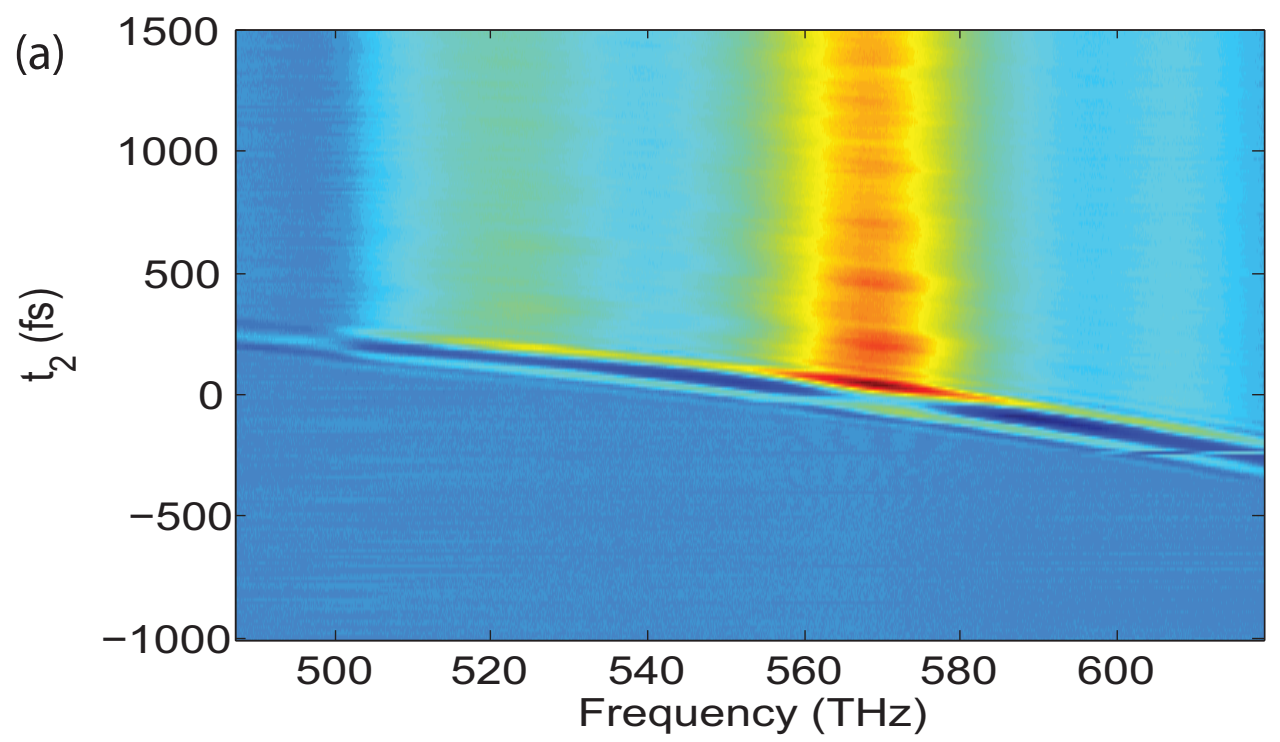

(b)

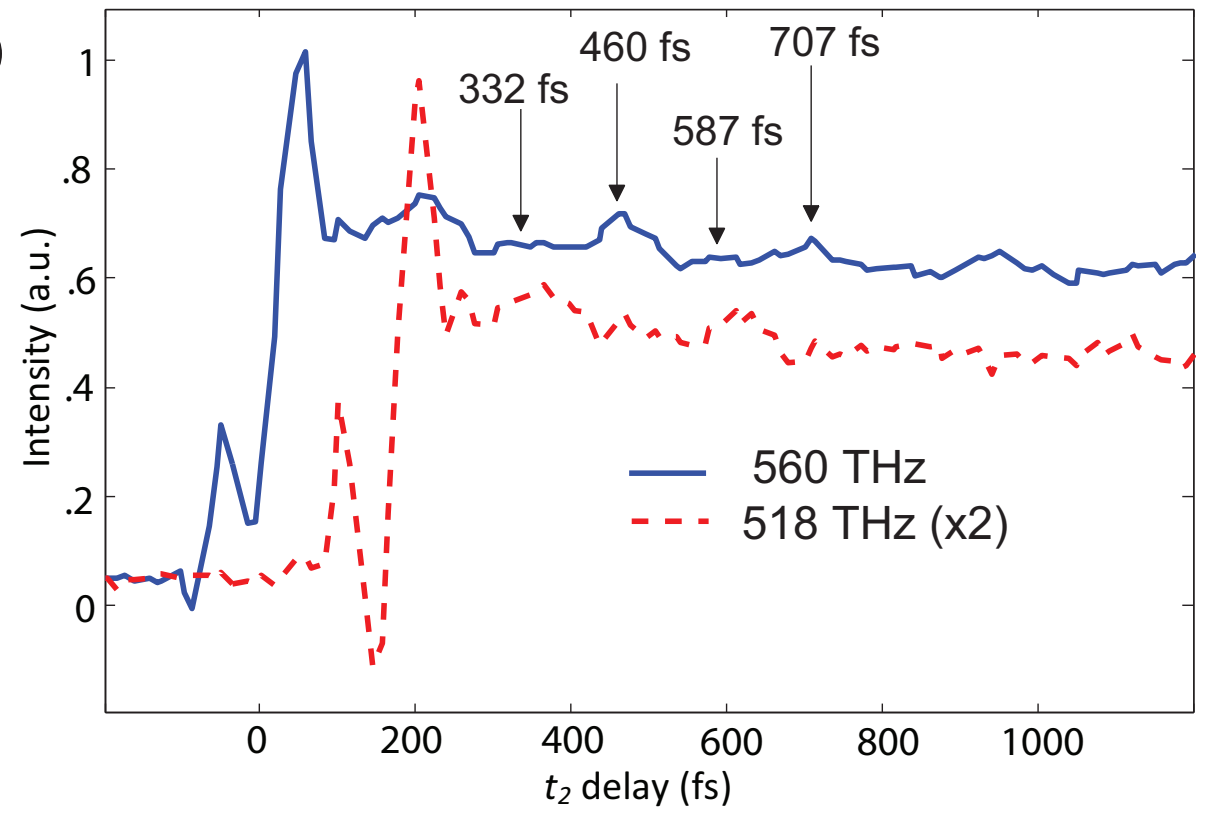

Figure 3.17: Pump-probe data for PERY in DMSO. (a) Frequency-resolved pumpprobe data for PERY in DMSO. (b) Slices from the pump-probe data at detection frequencies of $560 \mathrm{Thz}$ and $518 \mathrm{THz}$, clearly showing the 240 fs oscillations. 


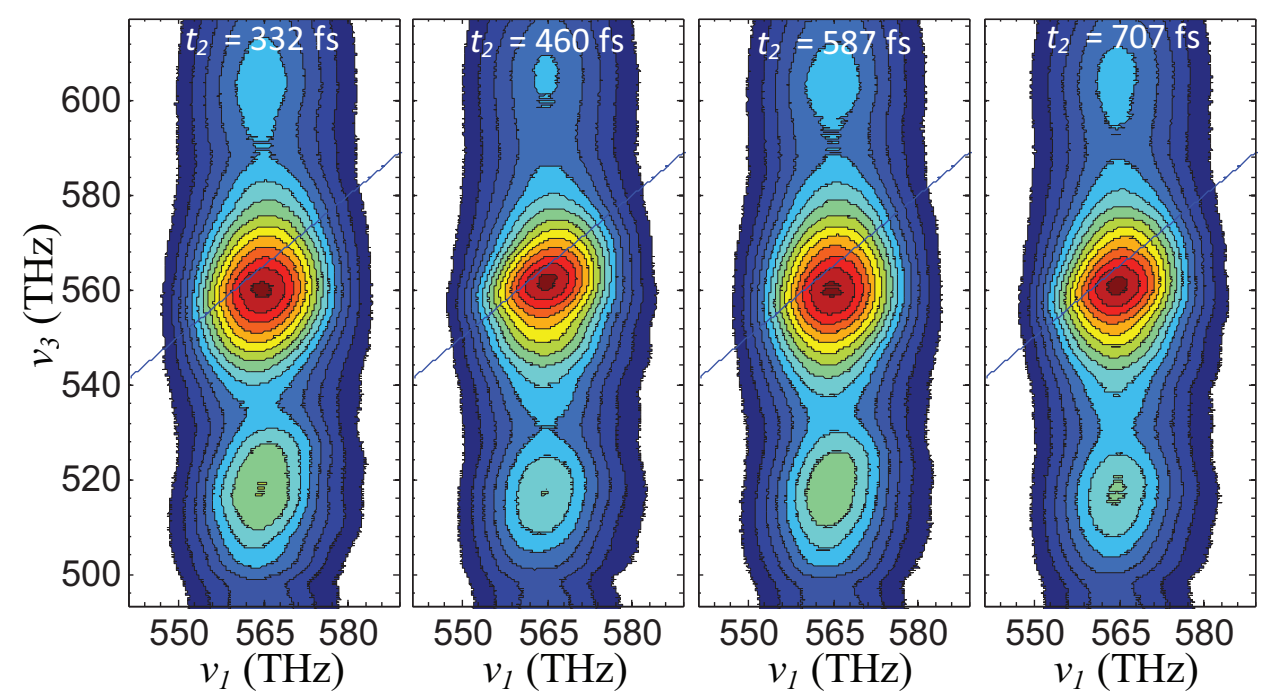

Figure 3.18: Absorptive 2D spectra of PERY in DMSO for four different waiting times, $t_{2}$.

in the 2D spectra of Figure 3.18(a). The lowest-frequency cross peak is a result of emission into the first excited vibrational ground electronic state, while the highestfrequency cross peak arises from the common ground state between the $0 \longrightarrow 0$ and $0 \longrightarrow 1$ transitions.

While subtle, a modulation in the ellipticity of the peaks is evident, as shown in Figure 3.19, where we plot the ratio of diagonal to antidiagonal FWHM of the peaks as a function of $t_{2}$. All three peaks exhibit ellipticity oscillations with the expected 240 fs period in accordance with coupling to the $139 \mathrm{~cm}^{-1}$ intramolecular mode. While 2D spectroscopy in the pump-probe geometry does not provide true dispersive spectra [14], we also observed an oscillation of the angle of the nodal line in the central peak in the imaginary $2 \mathrm{D}$ spectrum with the same $\sim 240$ fs period as shown in Figure 3.19. These results are consistent with those obtained by Nemeth et al., who observed strong modulation of the central peak in the absorptive spectrum and oscillations in the nodal line angle in the dispersive spectrum in a one-color 2D experiment [28]. They interpreted the modulation as arising from periodic changes in 


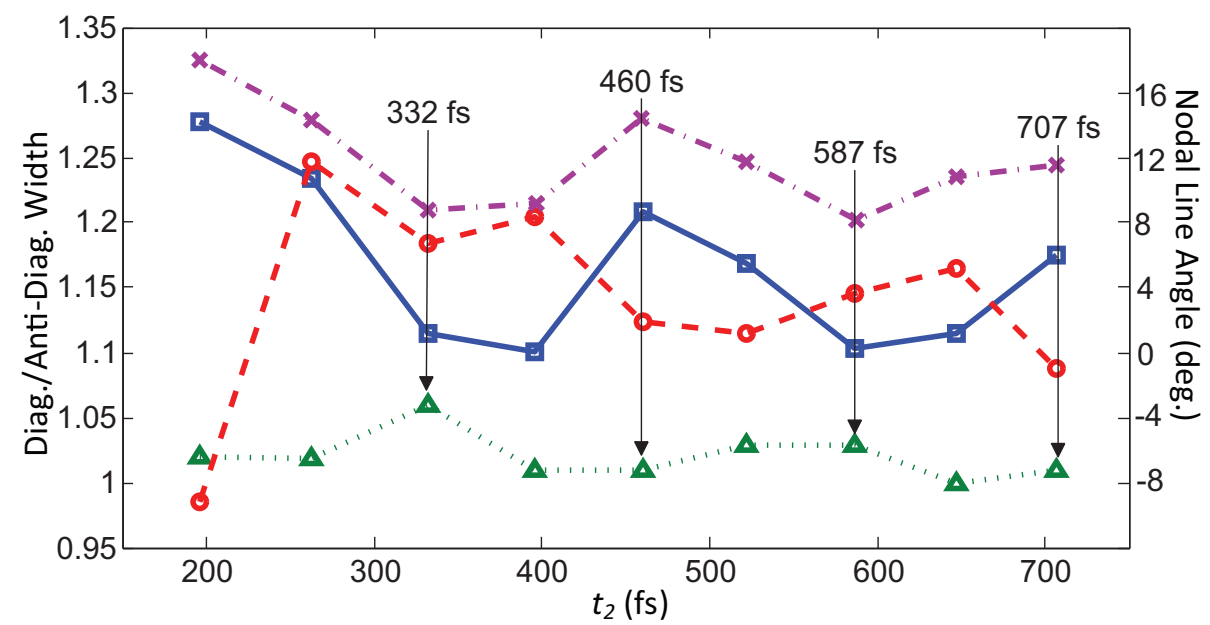

Figure 3.19: Ratio of diagonal to antidiagonal widths for the central peak (solid blue), lower peak (dashed red), and upper peak (dotted green), showing modulation with $\mathrm{a} \sim 240 \mathrm{fs}$ period. The nodal line in the dispersive spectra shows a similar oscillation (dashed-dotted purple).

the relative amplitude of rephasing and non-rephasing signal components caused by the coherent motion of the molecules vibrational degrees of freedom. A similar plot (not shown) of the peak amplitude as a function of $t_{2}$ exhibits 240 fs oscillations in the central peak, whereas the amplitude oscillations in the other peaks are difficult to discern.

The reduced time resolution arising from chirp on the continuum probe pulse presents some complications to the interpretation of the $2 \mathrm{D}$ spectra. As can be seen in the pump-probe data, the chirp results in a delay $t_{2}$ that is different for each probe frequency $\nu_{3}$. As determined by the frequency-integrated cross-correlation measurement, the variation in $t_{2}$ values within each $2 \mathrm{D}$ spectrum is $\sim 200 \mathrm{fs}$. This leads to distortions in our 2D spectra that we expect will affect both the lineshapes and the degree of vibronic modulation observed as a function of $t_{2}$. Future work will take into account the chirp of the probe pulse in modelling the $2 \mathrm{D}$ spectra and develop a correction algorithm to remove chirp-induced distortions. We note that vibrational 
wavepacket motion is frequently observed in electronic spectroscopy studies of chromophores [31]. The effect on 2D spectra must be taken into account for correct interpretation of lineshapes and dynamical processes.

\subsection{Comparison of methods}

The two implementations of the 2DES experiment in our lab complement each other in that each setup is strong in the aspects that are weak in the other. Between the two implementations, we have the capability to isolate most desired signals, to get faster data, or to reduce scatter as needed for the particular application. Depending on the sample to be studied and the specific requirements of the experiment, one 2DES implementation may be advantageous over the other. By maintaining both setups, we have wider capabilities. The salient differences between these two approaches are summarized in Table 3.1.

One of the chief advantages of the diffractive optic implementation is that the detected signal is background-free as a consequence of the non-collinear beam geometry. That and the fact that we heterodyne the signal pulse with a time-delayed local oscillator of controllable intensity, rather than the probe pulse, allows for easier isolation of weak signals apart from the strong excitation pulses. This makes the DO implementation ideal for samples of low optical density or weak interaction at the probe bandwidth, for instance. This sensitivity also means that a $2 \mathrm{D}$ spectrum with high SNR can be obtained in a single, continuous scan of the wedge motors, without averaging. This accelerates data collection immensely, as a 2D spectrum can be obtained in $\sim 5$-10 seconds, much faster than the $\sim 10$ minute acquisition time of the pulse-shaper implementation, which is hindered by the need to average over many laser shots and overhead associated with loading new waveforms into the pulse-shaper and periodically saving data from the CCD buffer.

Another point on which the two methods differ is that of the Feynman pathways 
collected. In the pump-probe geometry, the recovery of an absorptive spectrum is simple, since the rephasing and non-rephasing signals are collected collinearly and concurrently - the real part of the $2 \mathrm{D}$ spectrum automatically gives the absorptive component, removing the need for a separate scan. In the diffractive optic implementation, these two signals must be collected separately and then added together, the process of which can introduce distortions due to phase or timing errors. Timing of the $t_{1}$ delay is far simpler, since this is controlled directly by the pulse shaper and requires no additional calibration step. On the other hand, if one wishes to measure only the rephasing or non-rephasing pathway, it is much more direct in the DO implementation, since an additional scan is required to separate these contributions in the pump-probe geometry [19].

The two implementations differ in their handling of noise due to scatter. While the diffractive optic implementation relies on phase-matching and spatial filtering to remove the majority of scatter, chopping schemes must be implemented in some cases [8]. The pulse-shaper setup allows little spatial filtering, but the significant noise contributions can be removed through a simple scheme of chopping and phase cycling, as described above. A high optical density is often desirable in samples for the increase in SNR it provides. In the fully non-collinear case, however, it has been shown that OD values over roughly 0.3 can cause significant lineshape distortions in the $2 \mathrm{D}$ spectra [32]. When samples of high optical density are available, the pumpprobe geometry is far less susceptible to lineshape distortions caused by absorption, allowing for sample OD up to 1.2 [33].

Some researchers will also wish to have control of the polarization of the excitation pulses for the purposes of separating signals due to various dipolar combinations [34]. The diffractive optic implementation allows for arbitrary control of the polarization of each beam with the use of waveplates. With most pulse-shapers, only the polarization between the two beam pairs can be unambiguously controlled with reference to each 


\begin{tabular}{|c|c|}
\hline Pulse-shaper Approach & Diffractive-optics Approach \\
\hline$t_{1}=0$ known precisely & requires accurate measure of $t_{1}=0$ \\
\hline $\begin{array}{l}\text { rephasing and non-rephasing } \\
\text { signals collected simultaneously }\end{array}$ & $\begin{array}{l}\text { rephasing and non-rephasing signals } \\
\text { emitted in different directions and are } \\
\text { collected separately }\end{array}$ \\
\hline automatic phasing of $2 \mathrm{D}$ spectra & $\begin{array}{l}\text { phasing requires additional } \\
\text { pump-probe data collection }\end{array}$ \\
\hline excellent phase stability & good phase stability \\
\hline not background-free & background-free detection \\
\hline $\begin{array}{l}\text { slower collection due to } \\
\text { averaging and pulse-shaper } \\
\text { waveform loading time }\end{array}$ & rapid collection time \\
\hline
\end{tabular}

Table 3.1: A comparison of two implementations of 2DES

other, limiting the number of polarization schemes that can be readily implemented.

In short, both of these 2DES implementations have their merits and difficulties. Researchers would be urged to consider their experimental goals and weight the benefits of one method against the other on the basis of the specific study. It may often be the case that one's research goals would best be served by using both implementations. In our lab, we have the capability to perform both experiments. 


\section{Bibliography}

[1] A Stingl, M Lenzner, C Spielmann, F Krausz, and R Szipocs. Sub-1o-fs mirrordispersion-controlled ti-sapphire laser. Optics Letters, 20(6):602-604, MAR 15 1995.

[2] P. Maine, D. Strickland, P. Bado, M. Pessot, and G. Mourou. Generation of ultrahigh peak power pulses by chirped pulse amplification. Ieee Journal of Quantum Electronics, 24(2):398-403, 1988.

[3] T. Wilhelm, J. Piel, and E. Riedle. Sub-20-fs pulses tunable across the visible from a blue-pumped single-pass noncollinear parametric converter. Optics Letters, 22(19):1494-1496, 1997.

[4] R. L. Fork, O. E. Martinez, and J. P. Gordon. Negative dispersion using pairs of prisms. Optics Letters, 9(5):150-152, 1984.

[5] Charles G. Durfee, Jeff A. Squier, and Steve Kane. A modular approach to the analytic calculation of spectral phase for grisms and other refractive/diffractive structures. Optics Express, 16(22):18004-18016, OCT 272008.

[6] P. Baum and E. Riedle. Design and calibration of zero-additional-phase spider. Journal of the Optical Society of America B-optical Physics, 22(9):1875-1883, 2005 .

[7] M. L. Cowan, J. P. Ogilvie, and R. J. D. Miller. Two-dimensional spectroscopy using diffractive optics based phased-locked photon echoes. Chemical Physics Letters, 386(1-3):184-189, 2004.

[8] T. Brixner, T. Mancal, I. V. Stiopkin, and G. R. Fleming. Phase-stabilized twodimensional electronic spectroscopy. Journal of Chemical Physics, 121(9):42214236, 2004.

[9] L. Lepetit, G. Cheriaux, and M. Joffre. Linear techniques of phase measurement by femtosecond spectral interferometry for applications in spectroscopy. Journal of the Optical Society of America B-optical Physics, 12(12):2467-2474, 1995.

[10] NH Ge, MT Zanni, and RM Hochstrasser. Effects of vibrational frequency correlations on two-dimensional infrared spectra. Journal of Physical Chemistry A, 106(6):962-972, FEB 142002. 
[11] Yuan-Chung Cheng and Graham R. Fleming. Coherence quantum beats in two-dimensional electronic spectroscopy. Journal of Physical Chemistry A, 112(18):4254-4260, MAY 82008.

[12] M. Khalil, N. Demirdoven, and A. Tokmakoff. Obtaining absorptive line shapes in two-dimensional infrared vibrational correlation spectra. Physical Review Letters, 90(4), 2003.

[13] JD Hybl, AW Albrecht, SMG Faeder, and DM Jonas. Two-dimensional electronic spectroscopy. Chemical Physics Letters, 297(3-4):307-313, NOV 271998.

[14] SMG Faeder and DM Jonas. Two-dimensional electronic correlation and relaxation spectra: Theory and model calculations. Journal of Physical Chemistry A, 103(49):10489-10505, DEC 91999.

[15] JD Hybl, AA Ferro, and DM Jonas. Two-dimensional Fourier transform electronic spectroscopy. Journal of Chemical Physics, 115(14):6606-6622, OCT 8 2001.

[16] M. H. Cho, N. F. Scherer, G. R. Fleming, and S. Mukamel. Photon-echoes and related 4-wave-mixing spectroscopies using phase-locked pulses. Journal of Chemical Physics, 96(8):5618-5629, 1992.

[17] S. H. Shim, D. B. Strasfeld, Y. L. Ling, and M. T. Zanni. Automated 2d ir spectroscopy using a mid-ir pulse shaper and application of this technology to the human islet amyloid polypeptide. Proceedings of the National Academy of Sciences of the United States of America, 104(36):14197-14202, 2007.

[18] L. P. DeFlores, R. A. Nicodemus, and A. Tokmakoff. Two dimensonal fourier transform spectroscopy in the pump-probe geometry. Optics Letters, 32:29662968, 2007.

[19] J. A. Myers, K. L. M. Lewis, P. F. Tekavec, and J. P. Ogilvie. Two-color twodimensional fourier transform electronic spectroscopy with a pulse-shaper. Optics Express, 16(22):17420-17428, 2008.

[20] F. Verluise, V. Laude, Z. Cheng, C. Spielmann, and P. Tournois. Amplitude and phase control of ultrashort pulses by use of an acousto-optic programmable dispersive filter: pulse compression and shaping. Optics Letters, 25(8):575-577, 2000 .

[21] M. F. Emde, W. P. deBoeij, M. S. Pshenichnikov, and D. A. Wiersma. Spectral interferometry as an alternative to time-domain heterodyning. Optics Letters, 22(17):1338-1340, 1997.

[22] W. P. de Boeij, M. S. Pshenichnikov, and D. A. Wiersma. Heterodyne-detected stimulated photon echo: applications to optical dynamics in solution. Chemical Physics, 233(2-3):287-309, 1998. 
[23] W. P. de Boeij, M. S. Pshenichnikov, and D. A. Wiersma. Ultrafast solvation dynamics explored by femtosecond photon echo spectroscopies. Annual Review of Physical Chemistry, 49:99-123, 1998.

[24] S. H. Shim and M. T. Zanni. How to turn your pump-probe instrument into a multidimensional spectrometer: $2 \mathrm{~d}$ ir and vis spectroscopies via pulse shaping. Physical Chemistry Chemical Physics, 11(5):748-761, 2009.

[25] P. E. Tekavec, J. A. Myers, K. L. M. Lewis, and J. P. Ogilvie. Two-dimensional electronic spectroscopy with a continuum probe. Optics Letters, 34(9):1390-1392, 2009 .

[26] Kristin LM Lewis, Jeffrey A Myers, , Patrick Tekavec, and Jennifer P Ogilvie. Two-color two-dimensional fourier transform spectroscopy of energy transfer. In Ultrafast Phenomena XVI: Proceedings of the 16th International Conference, Palazzo dei Congressi Stresa, Italy, June 9-13, 2008, 2009.

[27] D. S. Larsen, K. Ohta, Q. H. Xu, M. Cyrier, and G. R. Fleming. Influence of intramolecular vibrations in third-order, time-domain resonant spectroscopies. i. experiments. Journal of Chemical Physics, 114(18):8008-8019, 2001.

[28] A. Nemeth, F. Milota, T. Mancal, V. Lukes, H. F. Kauffmann, and J. Sperling. Vibronic modulation of lineshapes in two-dimensional electronic spectra. Chemical Physics Letters, 459(1-6):94-99, 2008.

[29] SA Kovalenko, NP Ernsting, and J Ruthmann. Femtosecond hole-burning spectroscopy of the dye DCM in solution: The transition from the locally excited to a charge-transfer state. Chemical Physics Letters, 258(3-4):445-454, AUG 16 1996.

[30] Dassia Egorova, Maxim F. Gelin, and Wolfgang Domcke. Analysis of cross peaks in two-dimensional electronic photon-echo spectroscopy for simple models with vibrations and dissipation. Journal of Chemical Physics, 126(7), FEB 212007.

[31] Shaul Mukamel. Principles of Nonlinear Optical Spectroscopy. Oxford University Press, 1995.

[32] M. K. Yetzbacher, N. Belabas, K. A. Kitney, and D. M. Jonas. Propagation, beam geometry, and detection distortions of peak shapes in two-dimensional fourier transform spectra. Journal of Chemical Physics, 126(4):044511, January 2007.

[33] W. Xiong, D. B. Strasfeld, S. H. Shim, and M. T. Zanni. Automated 2d ir spectrometer mitigates the influence of high optical densities. Vibrational Spectroscopy, 50(1):136-142, May 2009.

[34] M. T. Zanni, N. H. Ge, Y. S. Kim, and R. M. Hochstrasser. Two-dimensional ir spectroscopy can be designed to eliminate the diagonal peaks and expose only 
the crosspeaks needed for structure determination. Proceedings of the National Academy of Sciences of the United States of America, 98(20):11265-11270, 2001. 


\section{CHAPTER IV}

\section{Photosystem II Studies}

In this chapter, I detail my methods of obtaining and analyzing data on the D1D2-cyt.b559 reaction center of plant Photosystem II. The chapter begins with an overview of how $2 \mathrm{D}$ and transient absorption data were obtained on these samples. In addition to merely stating what was done, this chapter will include some caveats for the physicist in dealing with biological samples that are susceptible to photodamage. The next section will present the data taken and will follow with various methods of post-processing and kinetic analysis. I will then discuss our observed kinetics in the context of previous spectroscopic investigations, summarizing our current thinking about the pathways and time scales of energy and charge transfer in the RC.

As discussed in Chapter, the reaction center $\mathrm{Q}_{y}$ band contains absorption bands for all chlorophyll and pheophytin molecules within the reaction center. The room temperature spectrum is shown in Figure 1.3. It is apparent that the linear absorption spectrum offers little hope for resolving the underlying subpopulations. It is well known that in the $\mathrm{Q}_{y}$ region spectral lineshapes narrow as temperature decreases, due to a reduction in dynamic inhomogeneities [1]. At $77 \mathrm{~K}$, the linear absorption spectrum becomes only slightly better resolved. At this temperature, the blue side of the spectrum exhibits a shoulder and the substructure begins to be resolvable, as seen in Figure 1.6. In order to better resolve the transitions and to improve sample 
stability, we obtained our 2D data on the reaction centers at $77 \mathrm{~K}$.

\subsection{Experimental Methods}

Reaction center samples were extracted from commercial spinach using the protocol described in Appendix C. To increase the optical density of the sample to a value appropriate for $2 \mathrm{DES}$, the samples were reconcentrated in a low salt buffer using a centrifuge filter (Millipore Amicon Ultra) composed of a regenerated cellulose membrane with a pore size of $10 \mathrm{kDa}$. This also allowed for reduction of the salt content in the sample buffer. After the reconcentration, samples were mixed with glycerol in a ratio of 2:1 glycerol to buffer. The glycerol is a popular cryoprotectant for low-temperature studies, as it mixes well with aqueous solutions and facilitates the production of an optically translucent glass instead of a crystalline solid at low temperatures.

Samples were loaded with a pipette into a custom sample cell (see Appendix D). Before loading the sample, the cryostat windows were treated with a solution of Rain$\mathrm{X}$ (chemically known as hexamethyldisilazane). This surface protectant causes the windows of the cell to become hydrophobic, thus reducing adhesion to the solvent. This becomes important at low temperatures: in untreated samples, the adhesive force exerts sufficient shear on the glass-state sample to fracture it into sub-millimeter size domains [2], making it very difficult to focus the laser beams in without extensive scatter and loss of signal. This effect is shown in Figure 4.1. With the surface treatment, only minor cracking occurs, and the domains of clear glass are quite large.

The sealed sample cell contains $30-40 \mu \mathrm{L}$ of sample solution at a path length of $350 \mu \mathrm{m}$ and an optical density of 0.5 at the absorption maximum of $675 \mathrm{~nm}$. While previous studies have shown that optical densities of this magnitude can cause distortion in 2D lineshapes under certain conditions in a boxcar geometry [3], recent studies have shown that in the pump-probe geometry, optical densities of up to 1.2 


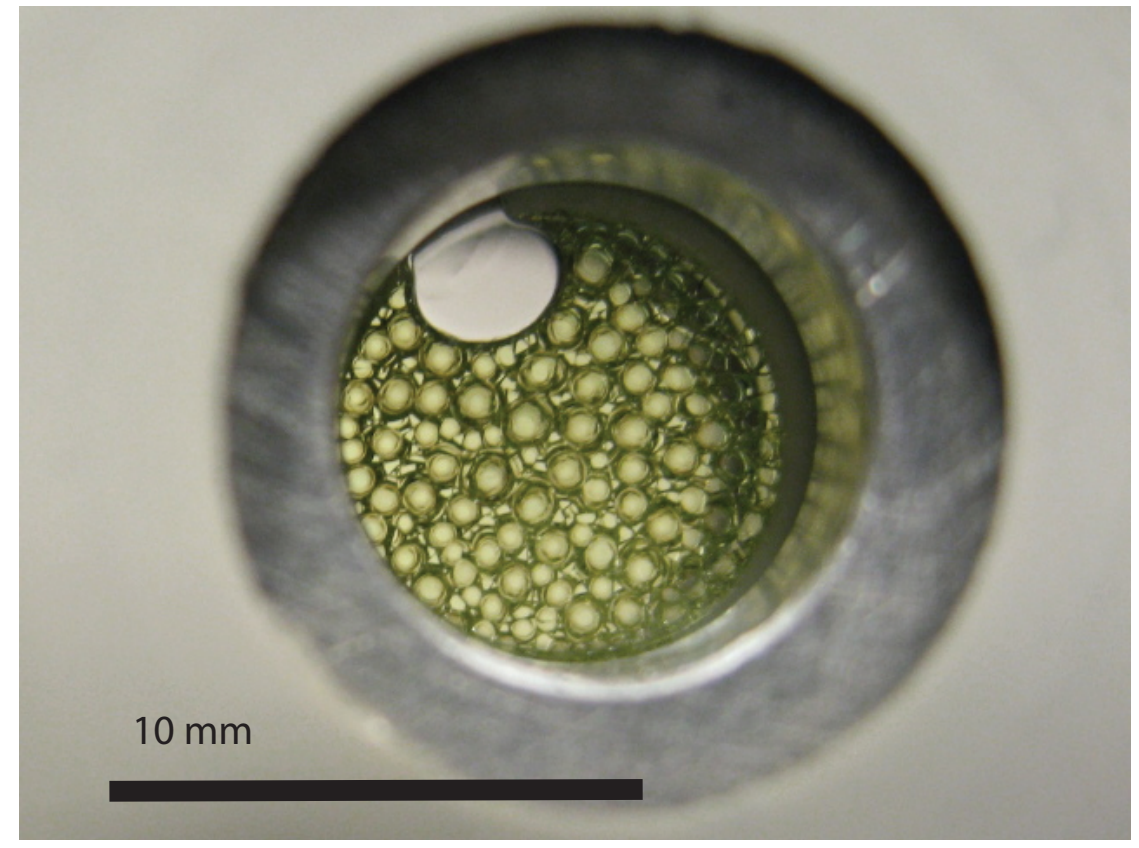

Figure 4.1: A sample of PSII in a sample cell with untreated windows. The fracturation occurs rapidly below the glass transition temperature, making data acquisition at $77 \mathrm{~K}$ impossible.

can be used without introducing significant distortion to the measured lineshapes [4].

The sample cell was secured in a cryostat (Oxford Instruments Microstat N), and a two-stage rotor pump (Varian DS102) was used to evacuate the cryostat. A flexible bellows was used in the vacuum line to discourage coupling of the pump vibrations into the cryostat. It is important to maintain a low pressure inside the cryostat throughout the experiment. The thermal conductivity through a diffuse gas is dependent on the pressure, temperature gradient, and path length: for the small volume of our cryostat and the large temperature gradient at $77 \mathrm{~K}$, a low pressure is essential for thermal isolation of the sample. Improper thermal isolation can lead to such problems as condensation of water vapor on the windows, difficulty stabilizing the sample temperature, or temperature gradients within the cryostat which can lead to improper measurements of temperature at the sample. It is recommended to maintain a sample pressure of $<10^{-3}$ mbar. Dry compressed air was flowed at a low 
rate over the front face of the cryostat to discourage the formation of condensation on the external window.

About 10 minutes following evacuation of the chamber, liquid nitrogen (LN) flow was initiated. A low-flow oil-free diaphragm pump pulls LN from the reservoir through an insulated transfer line to a coil around the sample cell. A regulator measures and controls the flow and expels the gaseous nitrogen exhaust. Temperature is monitored with a digital temperature controller connected to a sensor inside the cryostat. A heating element controlled by this device can be used to heat the sample manually or automatically (with a PID system). The temperature can be stabilized anywhere from $77-295 \mathrm{~K}$ to within an accuracy of roughly $0.5 \mathrm{~K}$ through a combination of heating and LN flow regulation. The heating element can also be used to raise the sample above room temperature if desired, though this was not done in any of the experiments presented here. A diagram of the cryostat system is shown in Figure 4.2.

For experiments on the $\mathrm{Q}_{y}$ transition, the NOPAs were both tuned to a center wavelength of $680 \mathrm{~nm}$. The bandwidth varied somewhat from day to day based on the precise tuning of the NOPAs, but values of 40-60 nm were typical for both beams. The spectra of both NOPA beams were tuned to completely cover the $\mathrm{Q}_{y}$ band, and the 2D spectra indicate that the signal amplitude was fully contained within the FWHM of both beams. Pump and probe spectra are shown in Figure 4.3. To minimize sample bleach, the probe pulse was attenuated with a variable neutral density filter to an energy of $10 \mathrm{~nJ}$, and the pump pulse was amplitude-shaped by the Dazzler to achieve an energy of $10 \mathrm{~nJ}$ per pump pulse. Pulse energies were measured with a Joulemeter (CVI Melles Griot 13SAM001) connected to a digital oscilloscope. Spot sizes were measured by moving a razor blade across the $\mathrm{x}$ or $\mathrm{y}$ axis of the beam with a micrometer stage and measuring the transmitted intensity with a fast photodiode. Spot sizes for the pump were $530 \mu \mathrm{m}$ by $440 \mu \mathrm{m}$. The probe size was $300 \mu \mathrm{m}$ by 190 $\mu \mathrm{m}$ at the focus. 


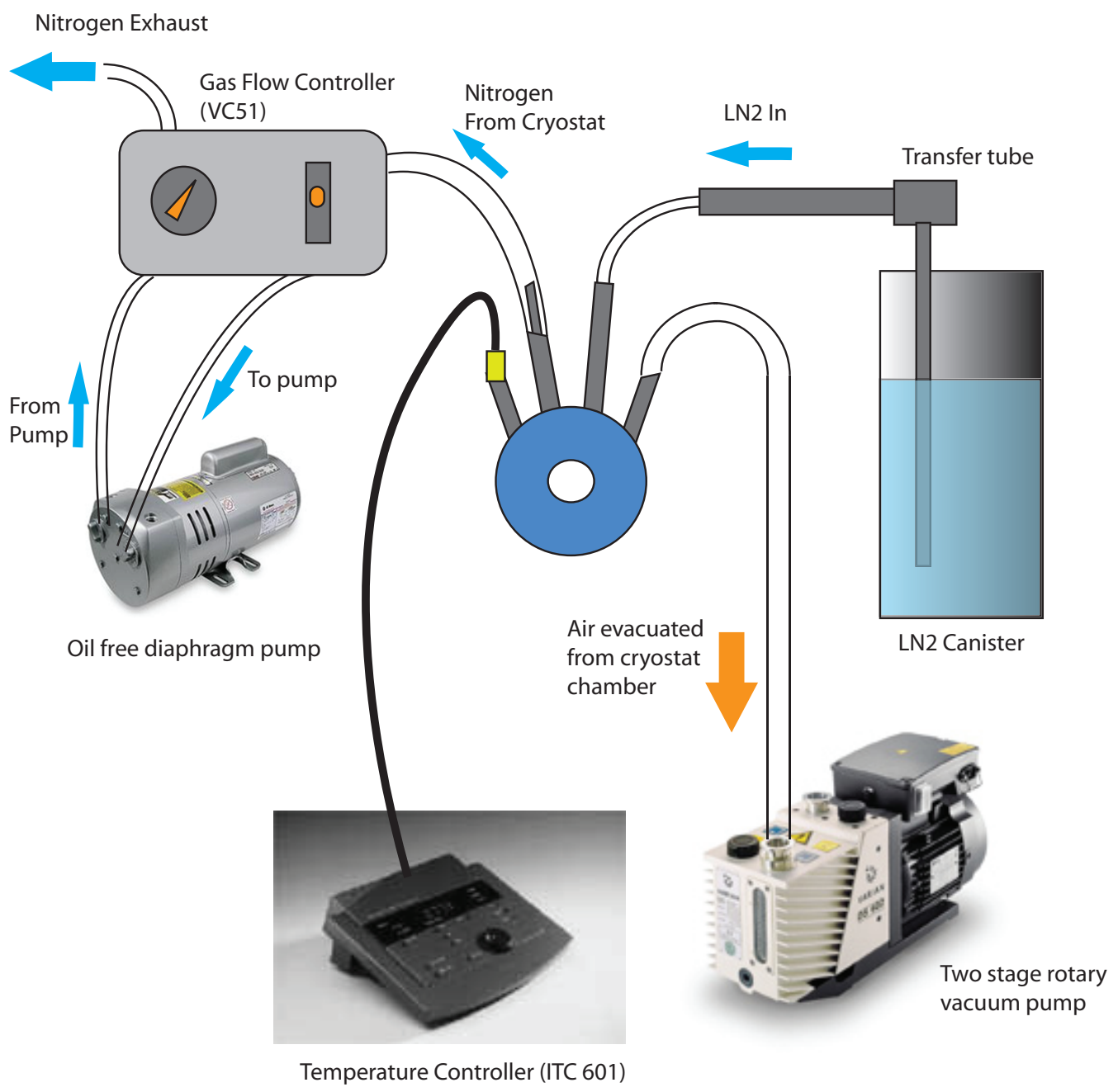

Figure 4.2: Diagram of the complete cryostat system, including the attached pumps, flow controller, temperature controller, liquid nitrogen canister, and transfer tube. Blue arrows indicate the flow of liquid and gaseous nitrogen. The orange arrow indicates the flow of gas from the chamber evacuation. 


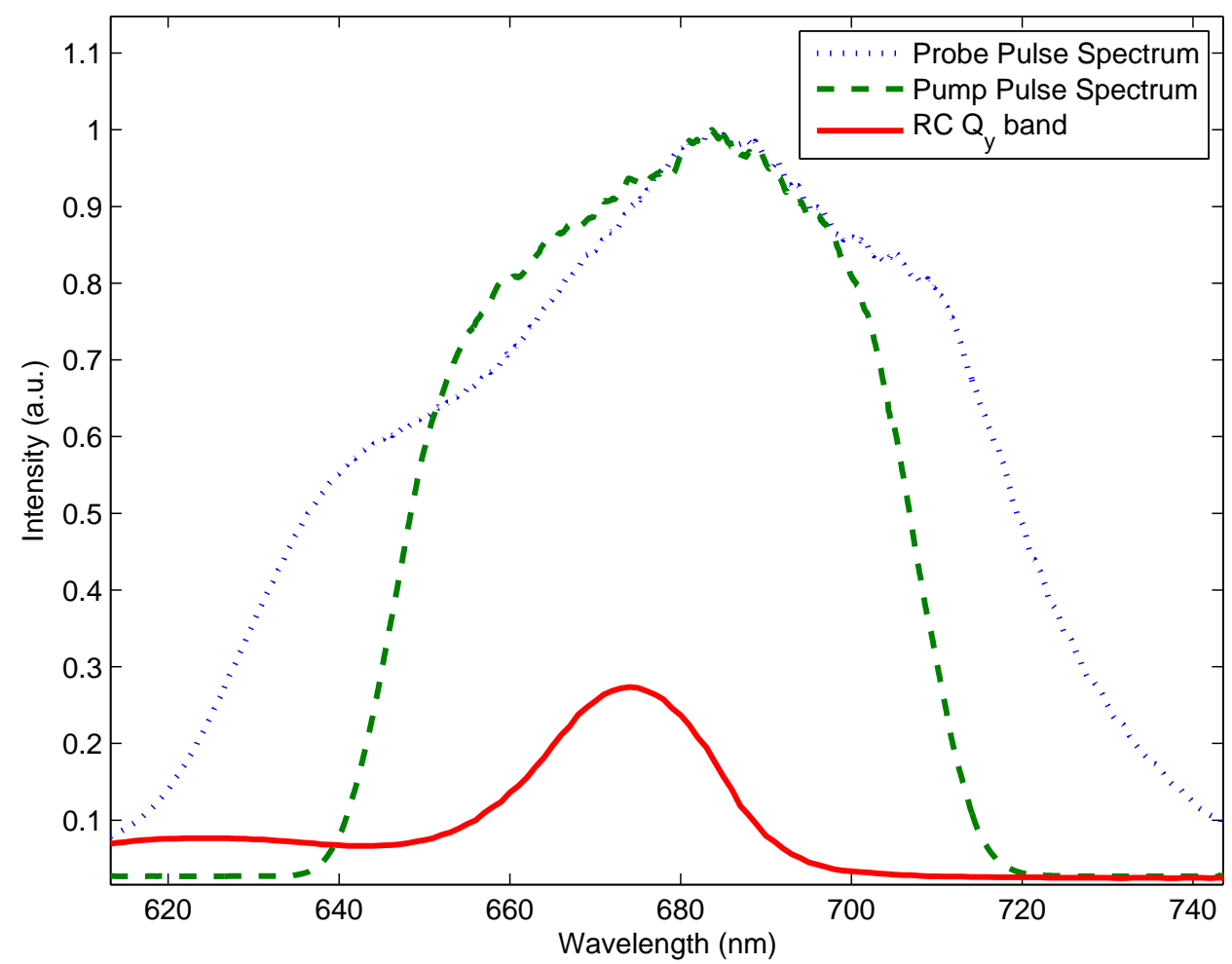

Figure 4.3: Spectra of pulses used in RC studies. The spectra of the pump pulses (dashed green line) and probe pulse (dotted blue line). The $\mathrm{RC} \mathrm{Q}_{y}$ band at room temperature is also shown (solid red line) and is clearly covered within the bandwidth of all the pulses. 


\section{Bleach Calculation}

In most ultrafast optical experiments, it is important to be aware of the sample bleach. In normal cases, a strong bleach can significantly reduce the signal amplitude by reducing the number of chromophores available for excitation from one shot to the next or across a series of measurements. Varying signal amplitude that is not due to real kinetic effects can cause misinterpretation of results if not corrected. In the RC samples, there is an additional concern: a single pulse which multiply excites the same RC can cause complications in the measurement due to exciton annihilation $[5,6]$. The higher the bleach, the more likely these events become, and the greater an effect it has on the data.

The sample bleach is defined as the number of photons $\left(N_{p h}\right)$ absorbed per reaction center in the focal volume $\left(N_{R C}\right)$ :

$$
B=\frac{N_{p h}}{N_{R C}}
$$

$N_{p h}$ is the ratio of the absorbed pulse energy $\left(E_{a b s}\right)$ and average photon energy $\left(E_{p h}\right)$.

$$
N_{p h}=\frac{E_{a b s}}{E_{p h}}
$$

For a given optical density $(O D)$, pulse energy $\left(E_{\text {pulse }}\right)$, and center wavelength $\left(\lambda_{0}\right)$, these energies are calculated, respectively, as:

$$
\begin{aligned}
E_{a b s} & =E_{\text {pulse }}\left(1-10^{-O D}\right) \\
E_{p h} & =\frac{h c}{\lambda_{0}}
\end{aligned}
$$

where $h$ is Planck's constant and $c$ is the speed of light.

To find the number of reaction centers in the sample volume, we first need to find the sample concentration $(C)$. In the regime of low optical density, the OD varies 
linearly with concentration and path length.

$$
O D=\epsilon \cdot l \cdot C
$$

where $\epsilon$ is the molar extinction coefficient and $l$ is the path length, or thickness of the sample cell in our case. For a measured OD and known path length, we rearrange the terms:

$$
C=\frac{O D}{\epsilon \cdot l}
$$

The focal volume $\left(V_{f}\right)$ is based on the spot size and sample thickness. For an elliptical beam of semimajor axis $x$ and semiminor axis $y$,

$$
V_{f}=x \cdot y \cdot \pi \cdot l
$$

The final value for $N_{R C}$ is

$$
N_{R C}=N_{A} \cdot C \cdot V_{f}
$$

where $N_{A}$ is Avogadro's number. The final bleach is obtained by plugging the results of Equations 4.2 and 4.8 into Equation 4.1. It is given as a decimal and often expressed in the literature as a percent. For very high fluencies, it is possible to have a bleach $B>1$, which means that each pulse will multiply excite a fraction of the reaction centers within the focal volume.

For our data, the bleach was calculated to be roughly $3 \pm 2 \%$, low enough to avoid significant complications to the data from exciton annihilation [5]. 


\subsection{Details of Data Acquisition}

To elucidate kinetics on time scales spanning several orders of magnitude, spectra were sampled at $t_{2}$ values at a quasi-logarithmic spacing, from 0 - 150 ps. The coherence time, $t_{1}$ was scanned from 0 - 450 fs in 1 fs steps for each $2 \mathrm{D}$ spectrum, and the Dazzler pulse sequence was phase locked at $1500 \mathrm{~nm}$ to allow undersampling via the rotating wave approximation. To remove scatter, a phase-cycling scheme utilized four distinct phase values [7], and the probe was chopped at a frequency of $250 \mathrm{~Hz}$. Under these conditions, sixteen shots were combined to produce a single measurement of the signal without scatter. The duty cycle was 0.5 due to the chopping. Approximately 50 complete sets of spectra were taken for each $t_{1}$ delay. This level of averaging allows for a very high signal to noise ratio. The total time to obtain a $2 \mathrm{D}$ spectrum for a single $t_{2}$ delay under this scheme was roughly 12.5 minutes. The data taken for a given experiment, consisting of several $t_{2}$ values (usually 30-65 values) on a single sample were obtained over the course of some 20-30 hours.

In order to minimize signal degradation due to sample bleach or photodamage, the cryostat was translated to select a new sample volume after every $t_{2}$ value. Analysis of the optical density of the sample over the course of a $2 \mathrm{D}$ spectrum did not show any significant persistent bleaching over the course of a single scan. This was confirmed by the fact that linear spectra taken after the completion of the experiment showed virtually no difference when compared to those taken before the experiment. Fresh samples were used for each experiment, and the experiments were repeated at least three times to ensure reproducibility.

\subsection{Data Treatment}

Following processing of the data as discussed in Chapter 2.7, an additional treatment of the full data set was performed to ensure the most accurate analysis. 
To adjust for variance in amplitude due to long-term laser amplitude drift and factors involved in selecting different focal volumes, such as variable scattering conditions or micron-scale differences in sample position upon translation in the focal plane, we invoke the projection-slice theorem [8]. This required recording transient absorption measurements over the full range of $t_{2}$ values included in our $2 \mathrm{D}$ spectra. The $2 \mathrm{D}$ spectra were each integrated over the excitation axis $\nu_{1}$ and compared to the spectrally-resolved TA spectrum at the corresponding $t_{2}$ delay. The ratio of peak amplitudes at each $t_{2}$ value were recorded and then used to scale the $2 \mathrm{D}$ data, so that each 2D spectrum was adjusted by a single multiplicative factor. This type of treatment therefore should not affect the relative amplitude of features within a given spectrum and reduces amplitude fluctuations that complicate extraction of the kinetics.

In our experiment, the local oscillator pulse passes through the sample, so its spectrum is modified by the sample absorption. In heterodyne measurements, the local oscillator field modulates the signal field, as shown in Equation 3.3. For samples of high optical density, it is therefore necessary to correct the spectra by deconvolving $\left|E_{l o}(\omega)\right|$ from the detected signal. To do this, the local oscillator spectrum was measured by averaging over many measured linear spectra for large values of $t_{1}$, for which the desired signal is shown to be no longer present due to dephasing effects during the coherence time. To obtain $\left|E_{l o}(\omega)\right|$, the square root the intensity spectrum was taken. The 3D spectral matrix was then divided by this field along the $\nu_{3}$ direction. The qualitative effect of this was to shift several of the spectral features closer to the diagonal, as was expected, and to reduce an observed amplitude bias which had favored the red part of the spectrum. A comparison of corrected and uncorrected spectra at $t_{2}=28 \mathrm{fs}$ is shown in Figure 4.4 . 

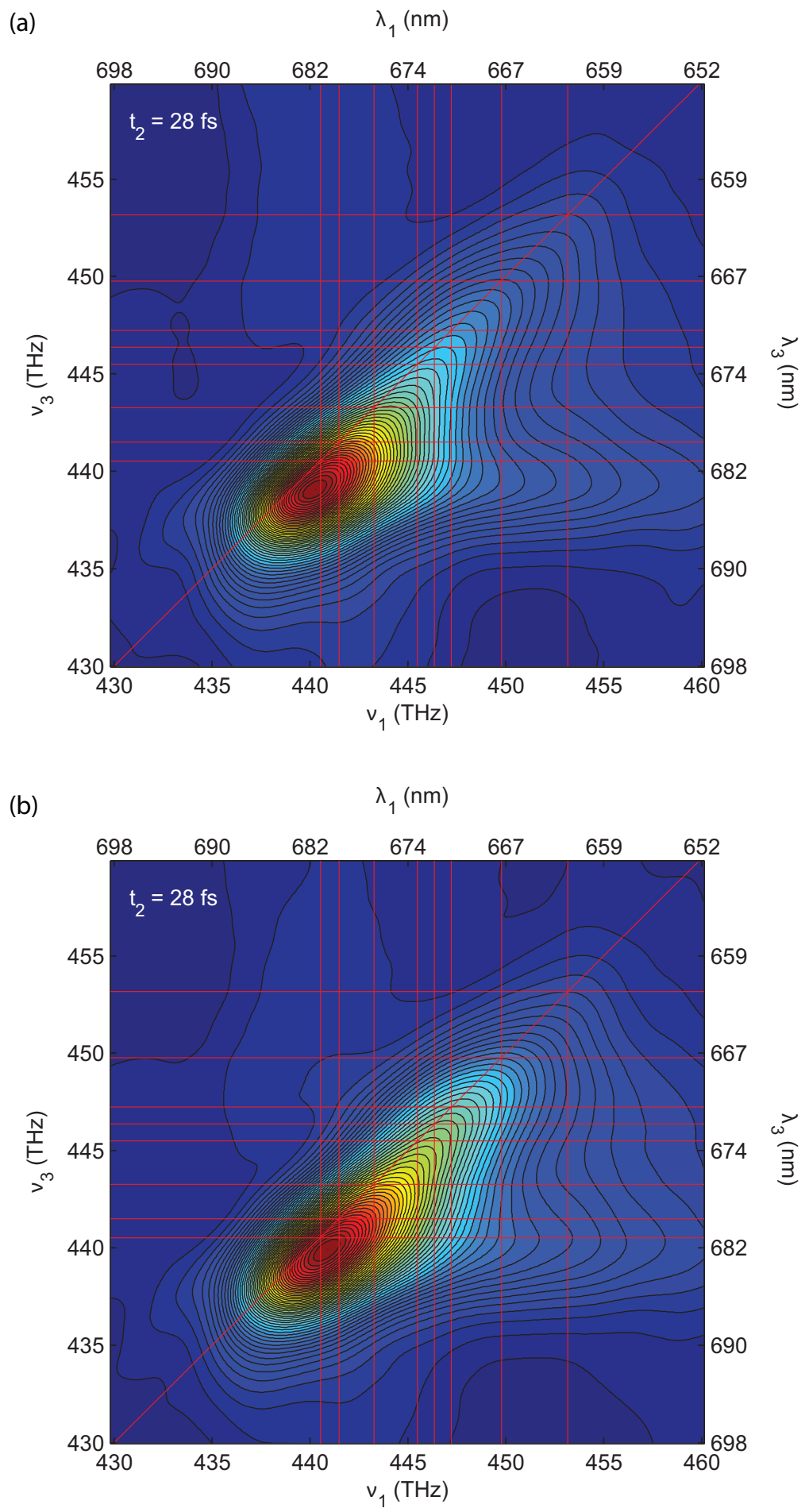

Figure 4.4: A comparison of uncorrected (a) and corrected (b) 2D spectra for the RC at $77 \mathrm{~K}$. 


\subsection{D Spectral Features of the PSII RC [9]}

Figure 4.5 shows the crystal structure of the PSII RC and a 2DES absorptive spectrum at $t_{2}=28 \mathrm{fs}$, with the exciton energies of the Novoderezhkin model [10] superimposed as a grid. The participation of the different pigments in the various exciton states is indicated by the colored squares. At $t_{2}=28 \mathrm{fs}$ contributions due to pulse overlap effects are largely complete and a strong cross-peak ( $\mathrm{CP} \#$ 1) below the diagonal is evident. A smaller cross-peak located symmetrically across the diagonal is also present ( $\mathrm{CP} \#$ 2). Diagonal features represent the direct excitation of excitons but may overlap with features of coupling or energy transfer between excitons of similar excitation energy. We note that the close spacing of the exciton levels makes it impossible to unambiguously assign excitonic coupling to all exciton pairs.

\section{Observations}

CP \# 1, located below the diagonal at all measured $t_{2}$ values, is a broad cross-peak that represents rapid downhill energy transfer between the higher energy excitons to excitons 1 and 2. CP \# 2 is a weaker cross-peak located across the diagonal from CP \# 1 and is observed even at shorter times, indicating coupling between excitons 1 and 2 and excitons 6,7 and 8 . We note that the close spacing of the exciton levels makes it impossible to unambiguously assign excitonic coupling to all three exciton pairs and that the diagonal feature obscures possible excitonic coupling between excitons 3,4 , and 5 to 1 and 2. Figures 4.6 and 4.7 show spectra at $t_{2}=215$ fs, 603 fs, 1.5 ps and 100 ps with the spectra normalized to the maximum of the $t_{2}=28 \mathrm{fs}$ spectrum. By $t_{2}=$ 215 fs CP \# 1 has grown substantially in magnitude. The $t_{2}=603$ fs spectrum looks similar with slightly broadened features and an overall decrease in amplitude. At $t_{2}=$ 1.5 ps the diagonal feature has shortened further as the highest energy exciton states transfer their energy downhill in energy, strengthening the relative amplitude of CP \# 1 compared to the diagonal. By $t_{2}=100$ ps the spectra show mainly an elongated 


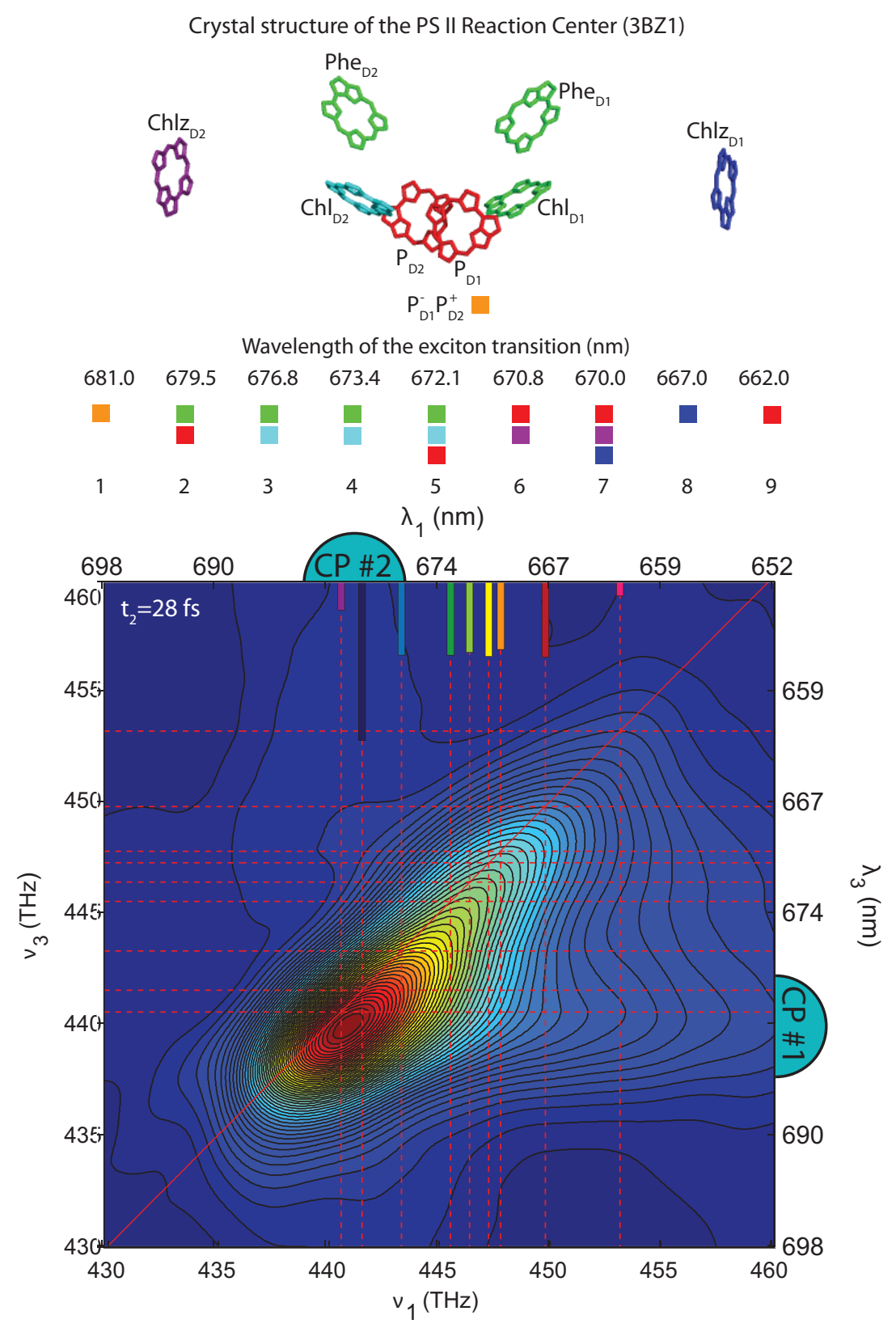

Figure 4.5: A 2DES spectrum of the D1D2-cyt.b559 reaction center at $77 \mathrm{~K}$ demonstrating the spectral features at $t_{2}=32 \mathrm{fs}$. The exciton lines from the model of Novoderezhkin et al. are overlaid as a grid, and constituent chromophores of these excitons are shown above. 
feature parallel to the excitation axis, indicating that most population resides in the low-energy exciton states 1 and 2 . The data do not change significantly between 100 ps and our longest recorded delay of $t_{2}=150 \mathrm{ps}$.

\subsection{Kinetic Analysis}

To date, the analysis of 2DES spectra has generally consisted of making comparisons between experimental and simulated 2DES spectra data based on modelling of the material response function. Other spectroscopic techniques attempt to elucidate the system dynamics through a rigorous mathematical treatment of the data, retrieving lifetime data through fits and clarifying the processes through a spectral dependence. In transient absorption spectroscopy, global-fitting analysis has been shown to be beneficial for extracting kinetics and revealing the spectral features displaying characteristic exponential decays via "decay associated spectra" [11]. It is our desire to provide a similar technique for systematic analysis of the dynamics inherent in experimental 2DES spectra which can be analyzed independently or in conjunction with modelling.

\section{Fitting Dynamics of Biological Systems}

Data on dynamical chemical and biological systems will often feature decay traces composed of a number of overlapping pathways, each with a different intrinsic rate constant. Functionally, each of these will contribute an exponential term $A_{i} e^{-t / \tau_{i}}$, where $A_{i}$ is the amplitude and $\tau_{i}$ the lifetime of the $i^{\text {th }}$ decay component. In some cases, growth terms are also included: these have a similar functional form $A_{i} e^{t / \tau_{i}}$, the only mathematical difference being the sign of the exponent. For growth terms, the time $\tau_{i}$ can usually be interpreted as a transfer time from another state. A onedimensional trace containing only exponential growth and decay terms will have the 

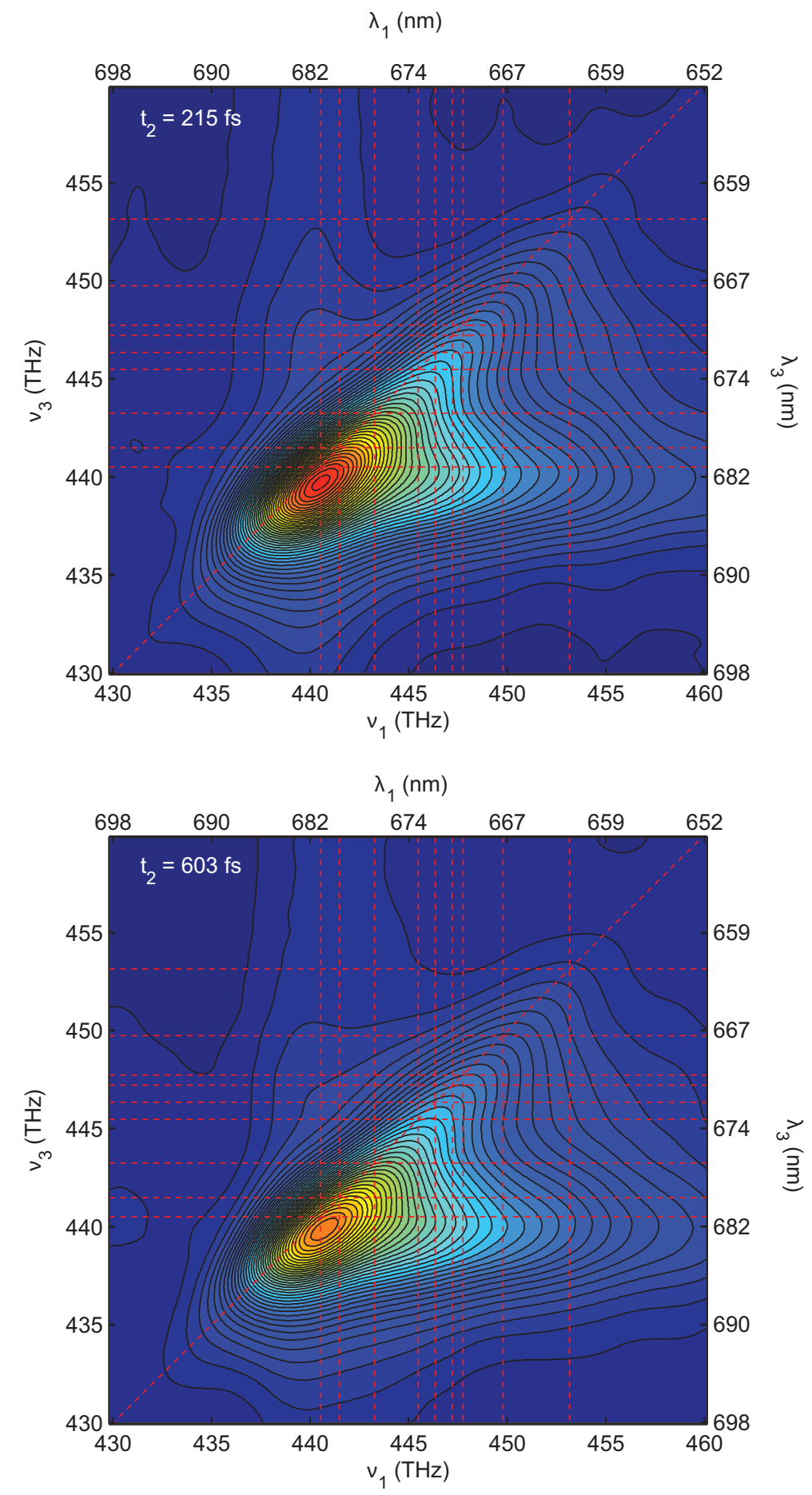

Figure 4.6: 2D Data on the D1D2-cyt.b559 reaction center at $77 \mathrm{~K}$ at $t_{2}=215 \mathrm{fs}$ (top) and $t_{2}=603 \mathrm{fs}$ (bottom) 

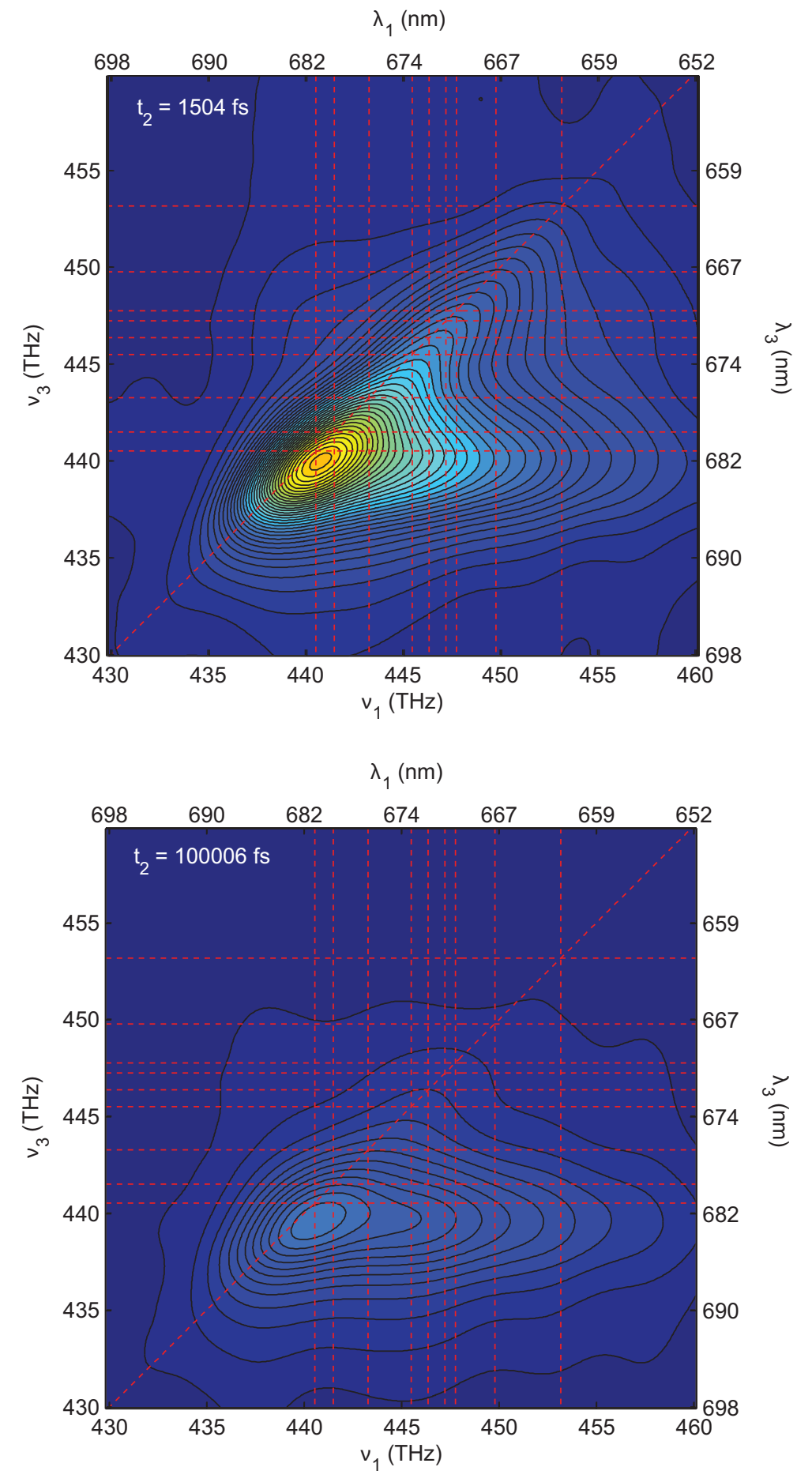

Figure 4.7: 2D Data on the D1D2-cyt.b559 reaction center at $77 \mathrm{~K}$ at $t_{2}=1.5 \mathrm{ps}$ (top) and $t_{2}=100 \mathrm{ps}$ (bottom) 
form

$$
A(t)=\sum_{i}^{N} A_{i} e^{-t / \tau_{i}}+\sum_{j}^{N} A_{j}\left(1-e^{-t / \tau_{j}}\right)
$$

where $i$ sums over the decay terms and $j$ over the growth terms for $\mathrm{N}$ different states. For many experiments, such as fluorescence lifetime studies and transient absorption, the trace $A(t)$ is the experimentally measured time series data. By fitting this data to a sum of exponentials, the researcher hopes to extract the amplitude and lifetime parameters. This is seldom a trivial matter.

The problem of multiexponential fitting is inherently nonlinear except in cases where the lifetimes are already well-known. The parameter phase space that fitting algorithms must explore is vast for even a biexponential curve and grows extremely complex as more terms are added. The most easily fit multiexponentials are those on which the lifetimes are all separated by an order of magnitude or more. When two lifetimes are close in value, the resulting fits may have difficulty distinguishing the two states and will often confuse them for a single lifetime of intermediate value. This can also happen for well-separated lifetimes if the sampling rate is insufficient to properly resolve them. The presence of noise in the data further complicates the problem.

\section{Multicomponent Fitting Techniques}

A common solution to recovering the lifetimes from a sum of exponentials is the application of the Levenberg-Marquardt algorithm for nonlinear least squares fitting [12]. In 1986, James and Ware published a method called the "exponential series method" (ESM) for analysis of single photon fluorescence decay data [13]. This method involves fitting the data to a series of many $(\sim 15-200)$ exponential terms with logarithmically spaced lifetimes. Because the lifetimes are held fixed and only the amplitudes are used as fit parameters, this becomes a linear problem. This particular method has been used by the Holzwarth group on narrowband transient 
absorption data [14], as well as in spectrally resolved data [15] in which "lifetime distribution maps" display a map of fitted lifetime distributions as a function of probe frequency. However, this method has failed to gain general use in the ultrafast spectroscopy community, perhaps due to the difficulty in properly constraining the parameter search to avoid non-physical solutions. Interestingly, Satzger and Zinth have used a similar method not to extract lifetimes but rather in an effort to provide a more intuitive visualization of raw TA data for the purpose of determining the number of well-separated lifetime components before performing any sort of fitting $[16]$.

The method of singular value decomposition (SVD) is often used to recover the components in spectrally-resolved decay data. While this is indeed a powerful method, it is not ideal for study of the RC. In SVD, a matrix of data in time vs. frequency is diagonalized to yield eigenvalues, which provide the decay rates, and eigenvectors, which give the associated spectral features. It must be stressed that SVD does not recover actual spectral components but rather obtains linear combinations of these. For a system with congested spectral features, such as the $\mathrm{RC}$, the eigenspectra will be sums and differences of the similar spectral components, the latter of which can easily be mistaken for noise and discarded due to its relatively low amplitude [17].

In transient absorption spectroscopy, global-fitting analysis has been shown to be beneficial for extracting kinetics and revealing the spectral features displaying characteristic exponential decays via "decay associated spectra" (DAS) [11]. Here we apply a similar analysis to $2 \mathrm{D}$ electronic spectra, producing analogous $2 \mathrm{D}$ decay associated spectra (2D DAS) to reveal the excitation and detection frequency dependence of energy and charge transfer processes in the PSII RC. A key difference between our approach and global analysis is that we used unconstrained time constants and each frequency-frequency trajectory in the $2 \mathrm{D}$ spectrum was fit independently to highlight the heterogeneous dynamics revealed by the excitation dependent information 
in 2DES data. We note that our method is similar in spirit to the lifetime distribution approach used by Holzwarth et al. [15].

\section{D DAS: Fitting and Displaying Frequency-Frequency Correlated Lifetime Distributions from 2DES Data}

We fit each excitation/detection frequency point in our 2D spectra to a sum of four exponential decay processes. Our choice of four exponentials is consistent with previous transient absorption studies at $77 \mathrm{~K}$ [18], as well as analysis of the residuals of fits to our data using fewer or more than four exponentials.

For a three-dimensional time series of absorptive $2 \mathrm{D}$ spectra $S\left(\nu_{1}, \nu_{3}, t_{2}\right)$, a given frequency point provides the total amplitude at a time $t_{2}$ corresponding to the physical processes that give rise to detection at frequency $\nu_{3}$, having excited the system at frequency $\nu_{1}$. This gives us an extra dimension of frequency selection over other spectroscopic methods which allows for a better isolation of energy and charge transfer as well as other dynamical processes.

Each one-dimensional trace is analyzed independently in order to prevent the introduction of any bias due from the fitting of neighboring frequency coordinates. Therefore, agreement between nearby points and smoothness of spectral features is a gauge of the quality of the 2D DAS rather than an imposed condition.

The kinetic trace $A\left(t_{2}\right)$ is fit by minimizing the least-squares variance

$$
\sum_{i=1}^{N}\left(A\left(t_{i}\right)-f\left(t_{i}\right)\right)^{2}
$$

where $f\left(t_{i}\right)$ is the retrieved multiexponential fit function at the $i^{\text {th }}$ population time value. For the sum of four exponentials used in these fits, the fit function is

$$
f\left(t_{i}\right)=\sum_{k=1}^{4} A_{k} e^{-t_{i} / \tau_{k}}
$$


where the index $k$ iterates over the exponential terms. The minimization was performed with an iterative, non-derivative based search algorithm in Matlab. To minimize the chance of settling into a local minimum in parameter space, each fit was performed 20 times with randomized start values for the $\tau_{k}$ distributed across four orders of magnitude. The $A_{k}$ are all initialized to the same value to avoid biasing the amplitudes. The function minimum (Equation 4.10) is evaluated for each of the 20 fits following termination of the search algorithm, and the parameters corresponding to the lowest overall function value are recorded for use in the 2D DAS.

For cases where growth occurs, the retrieved amplitude $A_{k}$ will be negative for that component. The fact that we use the functional form $A_{k} e^{-t_{i} / \tau_{k}}$ instead of the form $A_{k}\left(1-e^{-t_{i} / \tau_{k}}\right)$ used above to define exponential growth results in a DC offset of $A_{k}$ to the rest of the function, the amplitude of which is absorbed in the amplitude of the longest-lived component. This functional form was chosen because of the inability to retrieve reliable fits in the latter case and has no effect on the kinetics of the first three components.

The $R^{2}$ value is calculated for the final fit at each frequency-frequency coordinate, as shown in Figure 4.8. When constructing the 2D decay associated spectra, points that did not pass a sufficient criterion for goodness of fit are rejected. For the 2D DAS shown below, the threshold was $R^{2} \geq 0.9$, though fits to much of the data were considerably better than this. First, lifetimes are sorted and associated into separate 2D DAS with a clustering method: Amplitude-weighted distributions of the lifetime constants are plotted for all points in the spectrum and used to define components for the DAS, such that each component has a well separated distribution which approaches zero at the endpoints. The lifetimes and associated amplitudes for each component are plotted separately in the frequency-frequency domain. For well-behaved 2D DAS, the rates and amplitudes should be smoothly varying and continuous within a given spectral component, and different subpopulations may show 


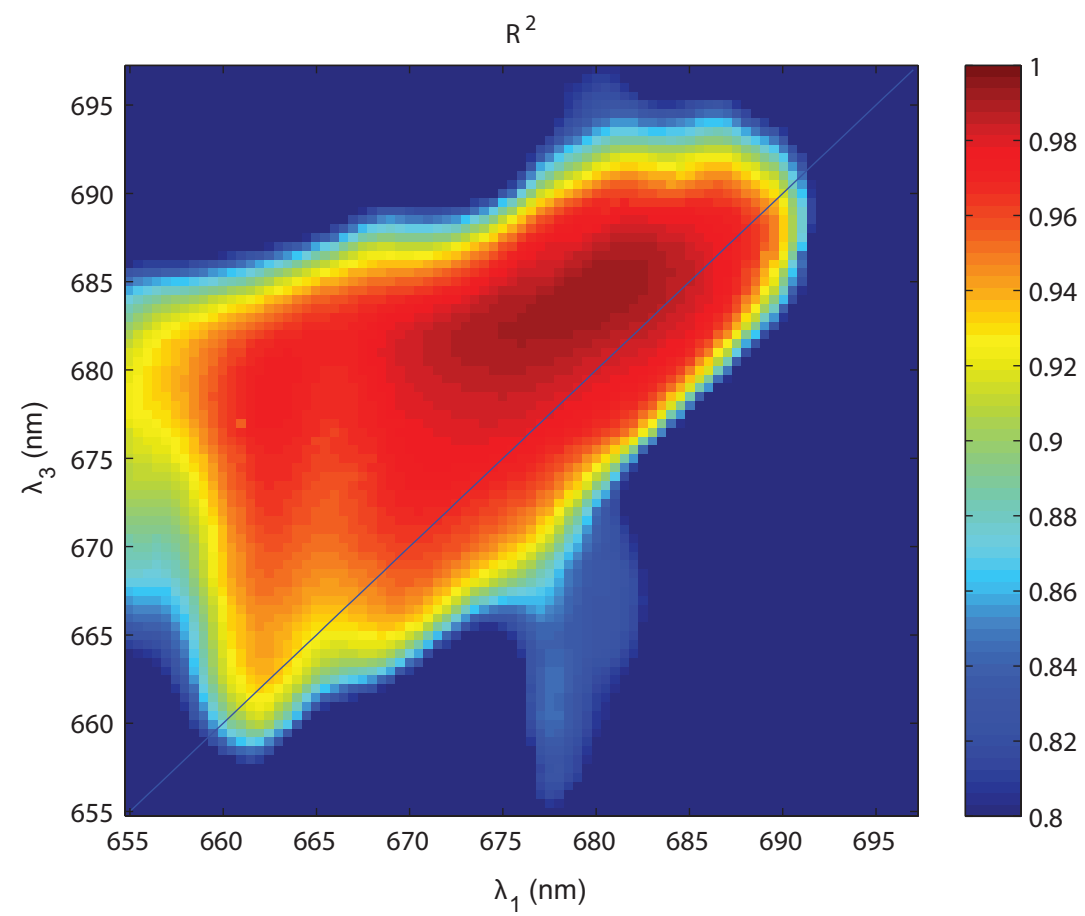

Figure 4.8: $\mathrm{R}^{2}$ values for the fits of the $\mathrm{RC}$ data to four exponentials.

sharp boundaries. This provides an easy and intuitive visualization of the different decay compartments and aids the spectroscopist in identifying subpopulations with distinct kinetics from neighboring components.

\subsection{Data Kinetics: 2D DAS of D1D2 data}

In order to obtain more detailed information on the kinetics of the samples, the data have been fit using the 2D DAS method, described above. Figures $4.9-4.12$ show the 2D DAS components for the D1D2-cyt.b559 complexes at $77 \mathrm{~K}$. The figures are plotted with axes spaced evenly in wavelength to facilitate comparison with other studies, which are usually evaluated on a wavelength axis. 2D DAS analysis was repeated on three or more sets of data taken on different days with fresh samples and yielded similar results. 


\section{Component I}

The fastest 2D DAS component (I) reveals rapid growth of $\mathrm{CP} \#$ 1, indicating fast downhill energy transfer. Excitons 8-6 transfer their energy to excitons 1 and 2 within $<\sim 100$ fs. Exciton 9 transfers energy to excitons 1 and 2 on a slower time scale, within $\sim 200$ fs. Energy transfer processes from excitons 3, 4 and 5 to excitons 1 and 2 are difficult to discern due to the overlapping diagonal feature in this region. Future polarization-dependent 2DES experiments [19] will suppress the diagonal feature to help reveal these dynamics. The elongated positive diagonal feature in component I indicates an overall decay of the population throughout the $\mathrm{Q}_{y}$ band as energy is redistributed. The negative diagonal feature at $690 \mathrm{~nm}$ can be attributed to pulse overlap effects that produce negative features that reduce the diagonal amplitude in this region. This interpretation is consistent with the retrieved sub 50 fs lifetime associated with this feature.

\section{Component II}

2D DAS component II corresponds to decays on the $\sim 1-3$ ps time scale. The largest amplitude associated with component II is concentrated along the diagonal in the region of excitons 1-3 with little contribution from the highest energy exciton states. The diagonal elongation indicates that the peak frequency red-shifts with increasing excitation wavelength. In this region the decay times are $\sim 2$ ps but gradually shorten to $\sim 1$ ps at higher wavelengths along the diagonal. We note a distinct longer-lived cross-peak of $\sim 3 \mathrm{ps}$ in the lifetime distribution is centered at $\sim$ $680 \mathrm{~nm}$ that likely results from population transfer from excitons 4-7.

\section{Component III}

2D DAS component III corresponds to decays on the $\sim 10-60$ ps time scale. The amplitude map for this component shows several distinct spectral features with 

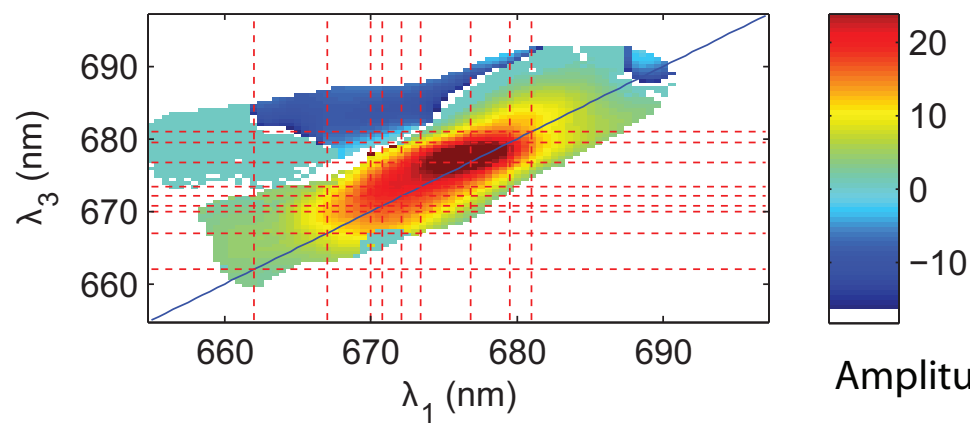

Amplitude (a.u.)
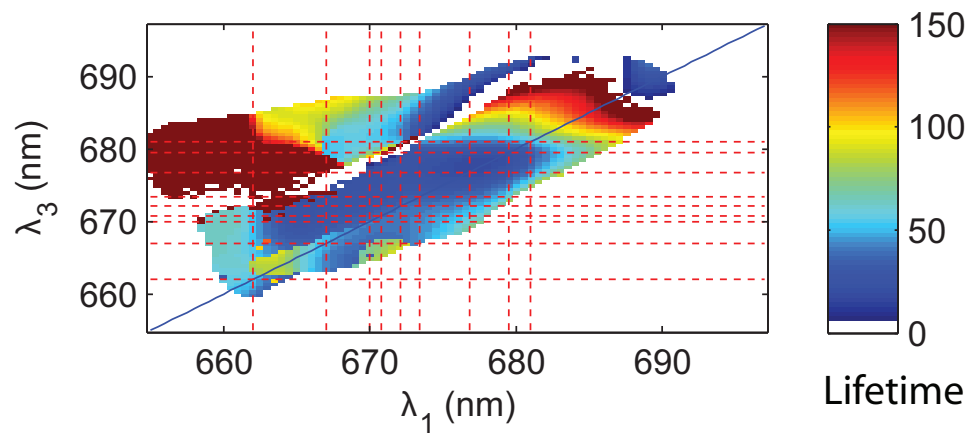

Lifetime (fs)

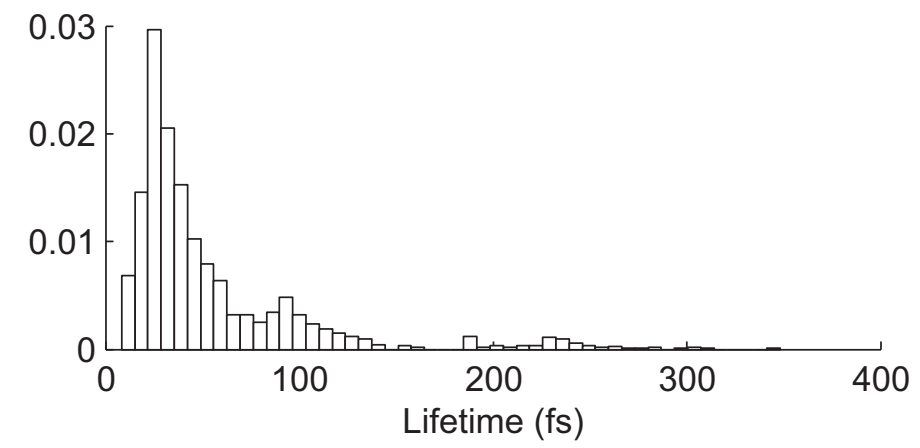

Figure 4.9: 2D DAS Component I showing kinetic processes for $t_{2}<400$ fs. Top: Amplitude, Middle: Lifetime map, Bottom: Histogram 

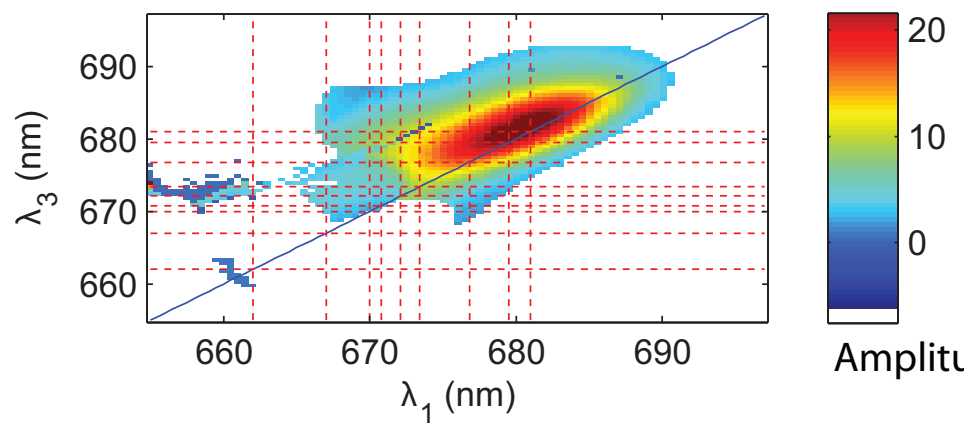

Amplitude (a.u.)
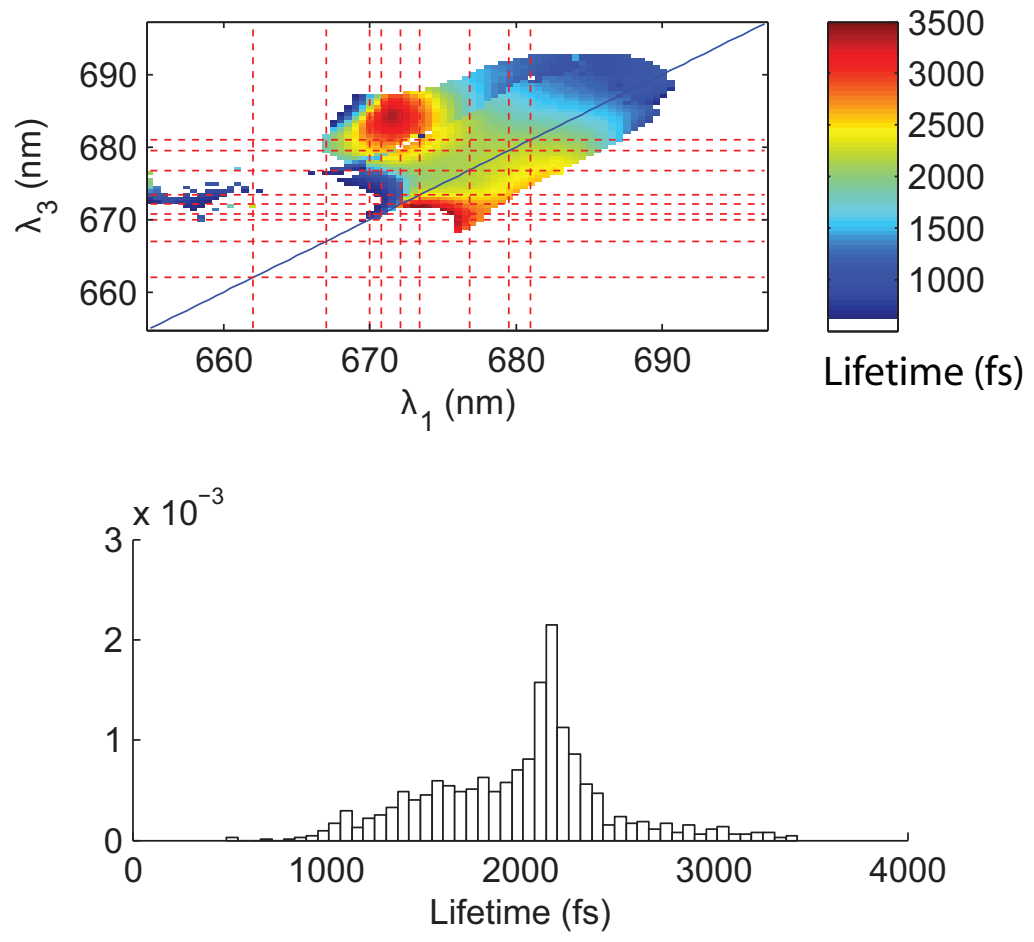

Figure 4.10: 2D DAS Component II showing kinetic processes for 500 fs $<t_{2}<4$ ps. Top: Amplitude, Middle: Lifetime map, Bottom: Histogram 
different associated lifetimes. Along the diagonal, excitons 6, 7 and 8 appear to decay with a lifetime of $\sim 6-8$ ps. A cross-peak indicating energy transfer from exciton 8 to excitons 1 and 2 decays within a similar lifetime. A longer-lived component along the diagonal near excitons 1 and 2 has a lifetime of $\sim 40-60$ ps.

\section{Component IV}

Finally, 2D DAS component IV corresponds roughly to the charge separated state, showing that most of the population resides in excitons 1 and 2 . The amplitude of this component was fit with a fixed exponential time constant of 2 ns. We found that the overall 2D DAS spectra were insensitive to the value of the radical pair decay when this time constant was larger than 2 ns.

\section{Discussion}

The D1D2-cyt.b559 reaction center operates under a series of dynamical processes, primarily excitation energy transfer (EET) between excitons and charge separation (CS). Energy transfer is generally downhill, funnelling excitations from a higher energy set of excitons to the lower energy states which are involved in primary charge separation.

The kinetics reported in the many spectroscopic studies of the RC were summarized in Tables 1.2 and 1.3. Here some of the most relevant studies for comparison with our work are briefly described.

Rapid, sub-ps energy equilibration between "blue" and "red" states [18, 20, 21, 22, $23,24,25,26]$ has been reported by several groups. Other studies [27] have attributed sub-ps processes to phonon relaxation or have failed to observe a sub-ps component at all [28]. Many transient absorption, hole-burning, and time-resolved fluorescence experiments have reported EET occurring in the tens of ps at low temperatures $[18,27,29,30,31,32,33,22]$ and near room temperature $[34,35,36,15,37,28,38$, 

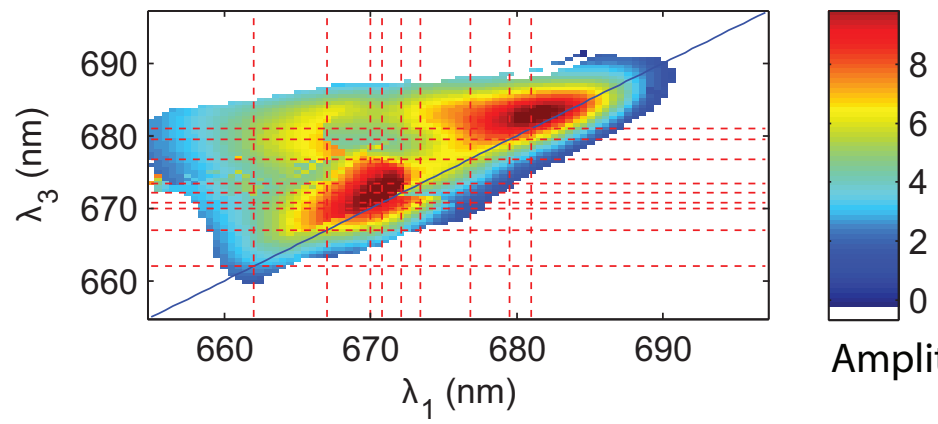

Amplitude (a.u.)
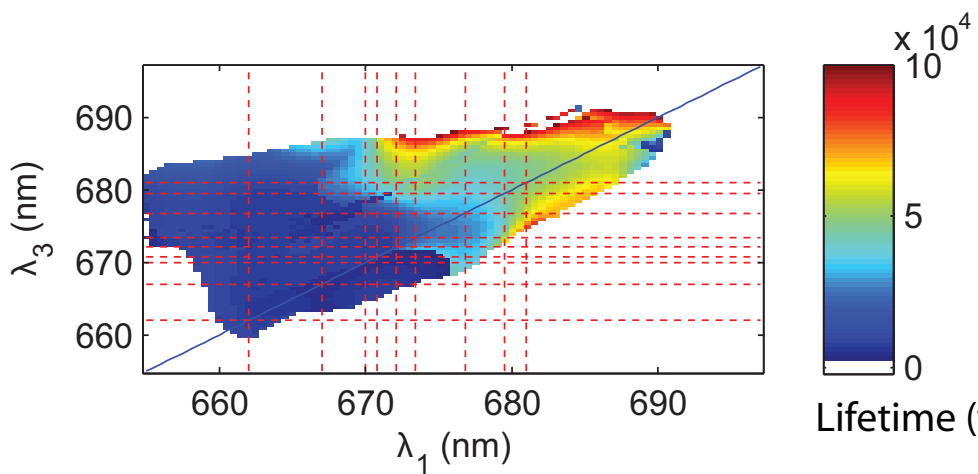

Lifetime (fs)

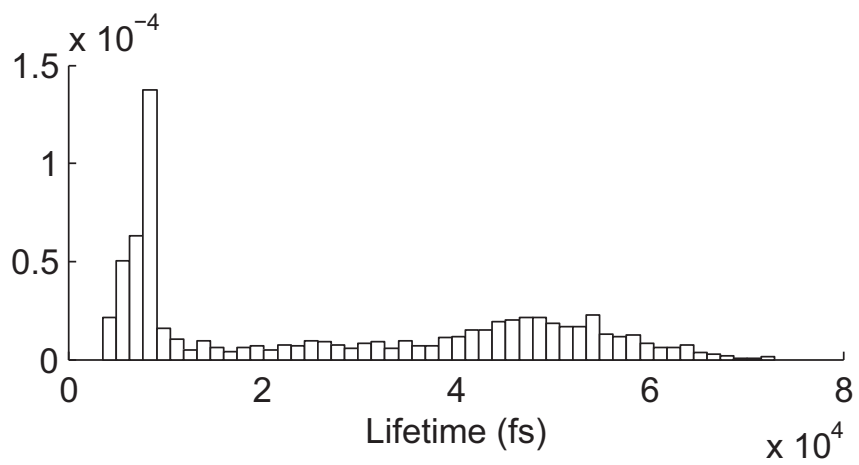

Figure 4.11: 2D DAS Component III showing kinetic processes for 4 ps $<t_{2}<100$ ps. Top: Amplitude, Middle: Lifetime map, Bottom: Histogram 

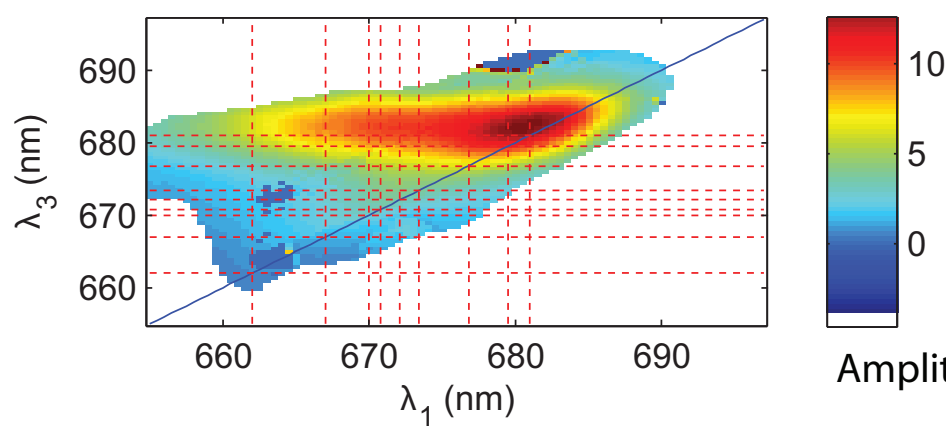

Amplitude (a.u.)
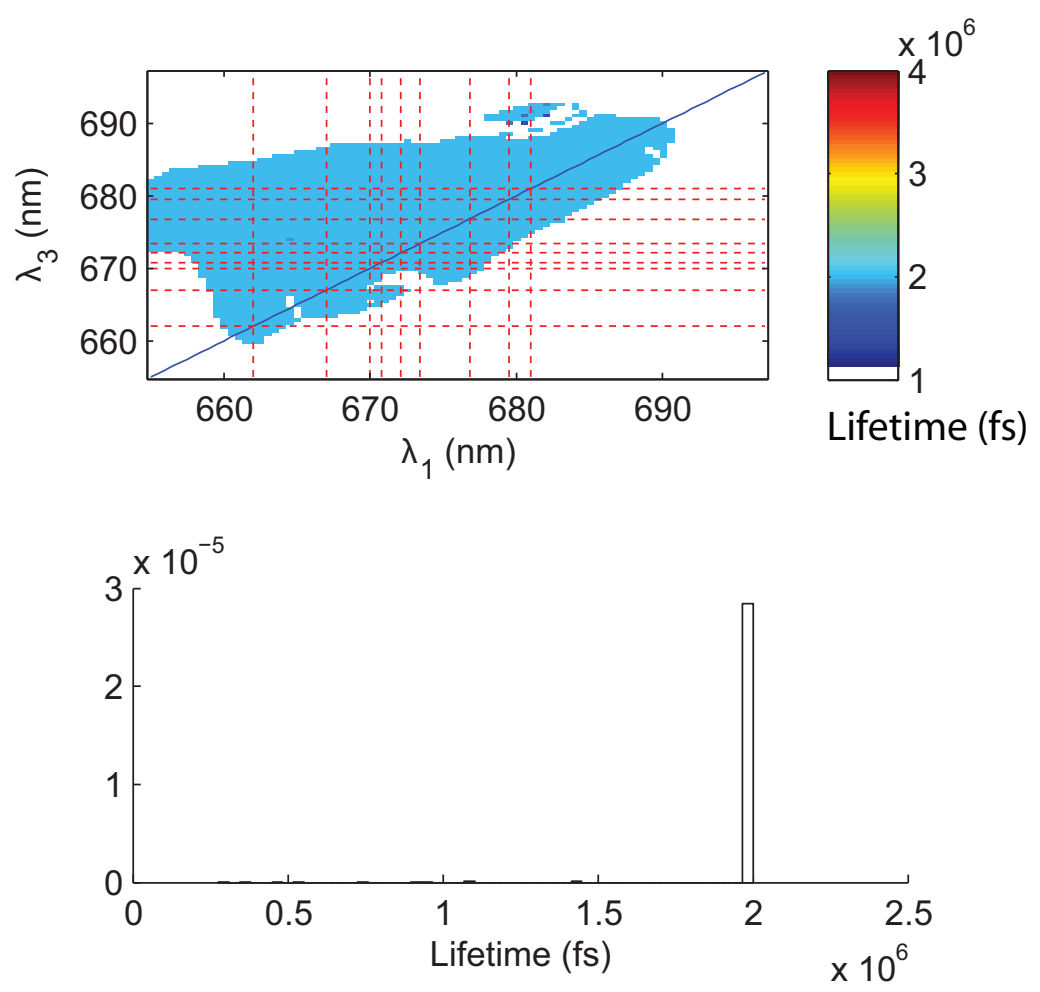

Figure 4.12: 2D DAS Component IV showing kinetic processes for $t_{2}>100 \mathrm{ps}$. The lifetime was fixed to $2 \mathrm{~ns}$ for this component. Top: Amplitude, Middle: Lifetime map, Bottom: Histogram 
24, 39, 40, 41]. When observed upon excitation on the blue side of the spectrum, this component is often assigned to slow energy transfer from the peripheral chlorophylls $\left(\mathrm{Chl}_{z}\right)$ to the red-absorbing excitons. At excitations near $680 \mathrm{~nm}$, this is sometimes posited as charge separation following rate-limited transfer from a degenerate "trap" state or a secondary charge transfer process.

While some earlier studies reported primary charge separation near 20 ps [24, 42, 43, 44, 5], many of the most recent studies have assigned primary CS to a faster component, between 1-3 ps [20, 21, 22, 30, 27, 15, 40, 41]. Several studies have also focused on identifying the specific chromophores involved in charge transfer. Recent work points to the assignment of $\mathrm{Chl}_{D 1}$ as the primary electron donor and the primary electron acceptor as $\operatorname{Pheo}_{D 1}[26,45,15,46,47,48]$. Romero et. al. [22] and Diner et al. [49] posit the existence of two CS pathways as opposed to a single CS pathway as described in Figure 1.5.

In our first 2D DAS component (I), we observe rapid growth of $\mathrm{CP} \# 1$, indicating fast downhill energy transfer. Excitons 8-6 transfer their energy to excitons 1 and 2 within $<\sim 100$ fs. Exciton 9 transfers energy to excitons 1 and 2 on a slower time scale, within $\sim 200$ fs. This exciton involves $\mathrm{P}_{D 1}$ and $\mathrm{P}_{D 2}$, and its transfer to exciton 1 is consistent with the rapid formation of the $\mathrm{P}_{D 1}^{-} \mathrm{P}_{D 2}^{+}$charge transfer state posited by Novoderezhkin et. al. to be accessed via sub 400 fs energy transfer [10]. A strong variation in lifetimes is observed across the $\mathrm{Q}_{y}$ band, with the states at the blue edge of the spectrum losing energy the most rapidly, consistent with the sub-400 fs feature observed upon excitation of the blue edge of the spectrum in narrow-band transient absorption experiments [18]. The lack of cross-peak growth below the diagonal reflects the lack of uphill energy transfer at $77 \mathrm{~K}$.

The excellent time resolution of our experiment and our ability to directly observe cross-peaks in the data provide us with a clear view of sub-ps kinetics. We note that our data helps resolve a discrepancy between two pulse photon echo (2PE) [26] and 
spectral hole-burning studies (SHB) [27]. In the 2PE experiments, a rapid $<100$ fs decay between 680-684 $\mathrm{nm}$ was attributed to energy transfer. This assignment was not supported by HB experiments that attributed the rapid decay to phonon relaxation processes. The rapid growth of $\mathrm{CP} \# 1$ provides definitive support for the energy equilibration idea, consistent with other work [18, 20, 21, 22, 23, 24, 25].

2D DAS component II corresponds to decays on the $\sim 1-3$ ps time scale. Spectral features in this time window in transient absorption and other studies have been attributed to charge transfer. Of particular interest in this time window are the features near $680 \mathrm{~nm}$ as a function of excitation wavelength that have been assigned to different radical pair states $[22,15]$. The largest amplitude associated with component II is concentrated along the diagonal in the region of excitons 1-3 with little contribution from the highest energy exciton states. The diagonal elongation indicates that the peak frequency red-shifts with increasing excitation wavelength, consistent with transient absorption studies using narrowband excitation [18].

We note a distinct longer-lived cross-peak of $\sim 3 \mathrm{ps}$ in the lifetime distribution is centered at $\sim 680 \mathrm{~nm}$ that likely results from population transfer from excitons 4-7. Based on the exciton model pigment assignments, this feature may reflect slower charge separation following energy transfer from the peripheral chlorophylls which have been assigned absorptions near $670 \mathrm{~nm}$.

A compartmental analysis will be necessary to test consistency of our data with the two charge separation scheme proposed by Romero et al. However, the distinct cross-peak feature in 2D DAS component II which shows a 3 ps decay may support the assignment of a second charge separation pathway. Additional evidence for this is presented in our preliminary studies on reduced samples, in which charge transfer pathways involving the pheophytin molecules are blocked (Appendix V). In these samples we observe the persistence of a slower $\sim 1$ ps component that is consistent with charge separation along the pathway that does not involve the pheophytin in 
the first CS step.

2D DAS component III corresponds to decays on the $\sim 10-60$ ps time scale. The amplitude map for this component shows several distinct spectral features with different associated lifetimes. Our observation of a 6-8 ps decay of excitons 6-9 along the diagonal in 2D DAS component III is reasonably consistent with transient absorption studies that showed $\mathrm{a} \sim 14 \mathrm{ps}$ component upon excitation at $670.5 \mathrm{~nm}$ and $672 \mathrm{~nm}$ [18]. Given the exciton assignments this is consistent with slow energy transfer from the $\mathrm{Chl}_{z}$ pigments followed by rapid CS.

The other prominent feature in this component, a 40-60 ps feature along the diagonal near $680 \mathrm{~nm}$, is reasonably consistent with proposed secondary charge transfer processes from radical pair intermediates to the charge separated $\mathrm{P}_{D 1}^{+} \mathrm{Pheo}_{D 1}^{-}$state $[22,26]$. It is also consistent with slow charge separation from a trap state nearly degenerate with excitons 1 and 2 and reported to have a similar lifetime in $77 \mathrm{~K}$ transient absorption studies [22, 18, 30].

Finally, 2D DAS component IV corresponds roughly to the charge separated state. This component was fit with a fixed exponential time constant of 2 ns. We found that the overall 2D DAS spectra were insensitive to the value of the radical pair decay when this time constant was larger than 2 ns. Since our maximum time delay was 150 ps we cannot comment on the presence of slower charge transfer processes on the hundreds of picosecond time scale. The recent work of Romero et. al. [22] shows a small blue shift on the hundreds of picoseconds to nanoseconds time scale, suggesting that at longer times our radical pair spectrum will be blue shifted by roughly 1-2 nm, bringing it in better agreement with the exciton model of Novoderezhkin et al.

We note that a recent two-color photon echo experiment on a bacterial reaction center showed long-lasting electronic coherence [50]. We did not observe similar coherences in the PSII RC at $77 \mathrm{~K}$ and believe this may reflect the smaller excitonic coupling and larger static disorder in the PSII RC. A more detailed discussion of this 
is included in Appendix A.

The most closely related work to our current 2DES study are the $77 \mathrm{~K}$ transient absorption (TA) studies of van Grondelle that examine the dependence on the excitation wavelength $[18,30,22]$ and the $1.3 \mathrm{~K}$ two-pulse photon echo (2PE) experiment by Prokhorenko et al. [26]. A previous accumulated photon echo experiment only observed very long-time dynamics [51]. Our study agrees well with the transient absorption work and observations from the 2PE study. Our work provides improved information about the excitation dependence of the kinetics with higher time resolution, enabling a clearer picture of the initial energy equilibration process.

An excitonic energy-transfer model, shown in Figure 4.13, displays the energy transfer between the exciton levels of Novoderezhkin et al. as directly observed in our 2D DAS measurements. A more complete model would include energy transfer involving excitons 3,4 , and 5 as well, but the present studies were not able to unambiguously resolve those kinetics due to the spectral congestion in that frequency region. We note that while we depict energy transfer from the higher energy excitons to excitons 1 and 2 in a single step, it is possible that intermediate transfers among excitons 9-3 occur. The severe overlap between exciton levels and the rapid $<100 \mathrm{fs}$ equilibrium process obscures there kinetics in our experiment.

Future polarization-dependent studies will give us the ability to suppress diagonal features and observe these cross-peaks directly. 


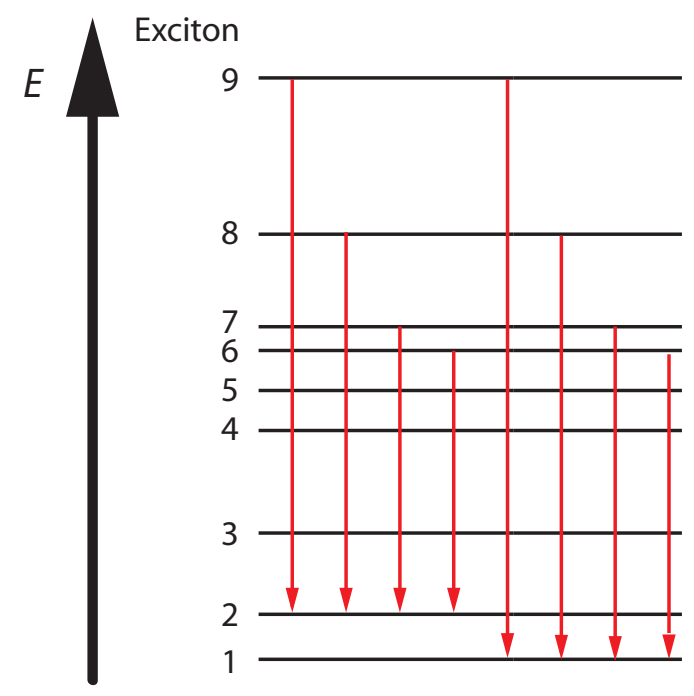

Figure 4.13: An excitonic energy-transfer model based on analysis of our data, using the model of Novoderezhkin et al. 


\section{Bibliography}

[1] LM Yoder, AG Cole, and RJ Sension. Structure and function in the isolated reaction center complex of photosystem ii: energy and charge transfer dynamics and mechanism. Photosynthesis Research, 72(2):147-158, 2002.

[2] Y. Rabin, P. S. Steif, K. C. Hess, J. L. Jimenez-Rios, and M. C. Palastro. Fracture formation in vitrified thin films of cryoprotectants. Cryobiology, 53(1):75-95, 2006.

[3] M. K. Yetzbacher, N. Belabas, K. A. Kitney, and D. M. Jonas. Propagation, beam geometry, and detection distortions of peak shapes in two-dimensional fourier transform spectra. Journal of Chemical Physics, 126(4):044511, January 2007.

[4] W. Xiong, D. B. Strasfeld, S. H. Shim, and M. T. Zanni. Automated 2d ir spectrometer mitigates the influence of high optical densities. Vibrational Spectroscopy, 50(1):136-142, May 2009.

[5] B Donovan, LA Walker, CF Yocum, and RJ Sension. Transient absorption studies of the primary charge separation in photosystem II. Journal of Physical Chemistry, 100(5):1945-1949, FEB 11996.

[6] M. G. Muller, M. Hucke, M. Reus, and A. R. Holzwarth. Annihilation processes in the isolated d1-d2-cyt-b559 reaction center complex of photosystem ii. an intensity-dependence study of femtosecond transient absorption. Journal of Physical Chemistry, 100(22):9537-9544, May 1996.

[7] S. H. Shim and M. T. Zanni. How to turn your pump-probe instrument into a multidimensional spectrometer: $2 \mathrm{~d}$ ir and vis spectroscopies via pulse shaping. Physical Chemistry Chemical Physics, 11(5):748-761, 2009.

[8] SMG Faeder and DM Jonas. Two-dimensional electronic correlation and relaxation spectra: Theory and model calculations. Journal of Physical Chemistry A, 103(49):10489-10505, DEC 91999.

[9] Jeffrey A. Myers, Kristin L. M. Lewis, Franklin Fuller, Patrick F. Tekavec, Charles F. Yocum, and Jennifer P. Ogilvie. Two dimensional electronic spectroscopy of the d1-d2-cyt b559 photosystem ii reaction center complex. Submitted to Journal of Physical Chemistry Letters, 2010. 
[10] V. I. Novoderezhkin, J. P. Dekker, and R. van Grondelle. Mixing of exciton and charge-transfer states in photosystem ii reaction centers: Modeling of stark spectra with modified redfield theory. Biophysical Journal, 93:1293-1311, 2007.

[11] I. H. M. van Stokkum, D. S. Larsen, and R. van Grondelle. Global and target analysis of time-resolved spectra. Biochimica Et Biophysica Acta-bioenergetics, 1657(2-3):82-104, 2004.

[12] D. W. Marquardt. An algorithm for least-squares estimation of nonlinear parameters. Journal of the Society For Industrial and Applied Mathematics, 11(2):431441, 1963.

[13] D. R. James and W. R. Ware. Recovery of underlying distributions of lifetimes from fluorescence decay data. Chemical Physics Letters, 126(1):7-11, 1986.

[14] V. I. Prokhorenko, D. B. Steensgaard, and A. F. Holzwarth. Exciton dynamics in the chlorosomal antennae of the green bacteria chloroflexus aurantiacus and chlorobium tepidum. Biophysical Journal, 79(4):2105-2120, 2000.

[15] M. G.; Reus M.; Nowaczyk M.; Sander J. \& Rogner M. Holzwarth, A. R.; Muller. Kinetics and mechanism of electron transfer in intact photosystem ii and in the isolated reaction center: Pheophytin is the primary electron acceptor. Proceedings of the National Academy of Sciences of the United States of America, 103(18):6895-6900, 2006.

[16] H. Satzger and W. Zinth. Visualization of transient absorption dynamics towards a qualitative view of complex reaction kinetics. Chemical Physics, 295(3):287-295, December 2003.

[17] A. K. Dioumaev. Evaluation of intrinsic chemical kinetics and transient product spectra from time-resolved spectroscopic data. Biophysical Chemistry, 67(1-3):125, 1997.

[18] HM VIisser, ML Groot, F van Mourik, IHM van Stokkum, JP Dekker, and R van Grondelle. Subpicosecond Transient Absorption Difference Spectroscopy on the Reaction-Center of Photosystem-II - Radical Pair Formation at 77-K. Journal of Physical Chemistry, 99(41):15304-15309, OCT 121995.

[19] E. L. Read, G. S. Engel, T. R. Calhoun, T. Mancal, T. K. Ahn, R. E. Blankenship, and G. R. Fleming. Cross-peak-specific two-dimensional electronic spectroscopy. Proceedings of the National Academy of Sciences of the United States of America, 104(36):14203-14208, 2007.

[20] S. R. Greenfield, M. Seibert, and M. R. Wasielewski. Time-resolved absorption changes of the pheophytin $\mathrm{q}(\mathrm{x})$ band in isolated photosystem ii reaction centers at $7 \mathrm{k}$ : Energy transfer and charge separation. Journal of Physical Chemistry B, 103(39):8364-8374, September 1999. 
[21] L. Konermann, I. Yruela, and A. R. Holzwarth. Pigment assignment in the absorption spectrum of the photosystem ii reaction center by site-selection fluorescence spectroscopy. Biochemistry, 36(24):7498-7502, June 1997.

[22] E. Romero, I. H. M. van Stokkum, V. I. Novoderezhkin, J. P. Dekker, and R. van Grondelle. Two different charge separation pathways in photosystem ii. Biochemistry, 49(20):4300-4307, 2010.

[23] JR Durrant, G Hastings, DM Joseph, J Barber, G Porter, and DR Klug. Subpicosecond Equilibration of Excitation-Energy in Isolated Photosystem-II Reaction Centers. Proceedings of the National Academy of Sciences of the United States of America, 89(23):11632-11636, DEC 11992.

[24] M. G. Muller, M. Hucke, M. Reus, and A. R. Holzwarth. Primary processes and structure of the photosystem ii reaction center .4. low-intensity femtosecond transient absorption spectra of d1-d2-cyt-b559 reaction center. Journal of Physical Chemistry, 100(22):9527-9536, May 1996.

[25] S. A. P. Merry, S. Kumazaki, Y. Tachibana, D. M. Joseph, G. Porter, K. Yoshihara, J. Barber, J. R. Durrant, and D. R. Klug. Sub-picosecond equilibration of excitation energy in isolated photosystem ii reaction centers revisited: Timedependent anisotropy. Journal of Physical Chemistry, 100(24):10469-10478, June 1996.

[26] V. I. Prokhorenko and A. R. Holzwarth. Primary processes and structure of the photosystem ii reaction center: A photon echo study. Journal of Physical Chemistry B, 104(48):11563-11578, 2000.

[27] V. Zazubovich, R. Jankowiak, K. Riley, R. Picorel, M. Seibert, and G. J. Small. How fast is excitation energy transfer in the photosystem ii reaction center in the low temperature limit? hole burning vs photon echo. Journal of Physical Chemistry B, 107(12):2862-2866, 2003.

[28] JPM Schelvis, PI van Nort, TJ Aartsma, and HJ van Gorkom. EnergyTransfer, Charge Separation and Pigment Arrangement in the Reaction-Center of Photosystem-II. Biochemica et Biophysica Acta-Bioenergetics, 1184(2-3):242250, MAR 81994.

[29] MR Wasielewski, DG Johnson, Govindjee, C Preston, and M Seibert. Determination of the Primary Charge Separation Rate in Photosystem-II Reaction Centers at 15-K. Photosynthesis Research, 22(1):89-99, OCT 1989.

[30] M. L. Groot, F. vanMourik, C. Eijckelhoff, I. H. M. vanStokkum, J. P. Dekker, and $\mathrm{R}$. vanGrondelle. Charge separation in the reaction center of photosystem ii studied as a function of temperature. Proceedings of the National Academy of Sciences of the United States of America, 94(9):4389-4394, April 1997. 
[31] D. Tang, R. Jankowiak, M. Seibert, C. F. Yocum, and G. J. Small. Excited-state structure and energy-transfer dynamics of 2 different preparations of the reaction center of photosystem-ii - a hole-burning study. Journal of Physical Chemistry, 94(17):6519-6522, August 1990.

[32] TA Roelofs, SLS Kwa, R van Grondelle, JP Dekker, and AR Holzwarth. Primary Processes and Structure of the Photosystem II Reaction-Center .2. LowTempterature Picosecond Fluorescence Kinetics of a D1-D2-Cyt-B-559 ReactionCenter Complex Isolated by Short Triton Exposure. Biochemica et Biophysica Acta, 1143(2):147-157, JUL 51993.

[33] A Freiberg, K Timpmann, AA Moskalenko, and NY Kuznetsova. Picosecond and Nanosecond Fluorescence Kinetics of Photosystem-II Reaction-Center and its Complex With CP47 Antenna. Biochemica et Biophysica Acta-Bioenergetics, 1184(1):45-53, FEB 81994.

[34] TA Roelofs, M Gilbert, VA Shuvalov, and AR Holzwarth. Picosecond Fluorescence Kinetics of the D1-D2-Cyt-B-559 Photosystem-II Reaction Center Complex - Energy-Transfer and Primary Charge Separation Processes. Biochemica et Biophysica Acta, 1060(3):237-244, NOV 71991.

[35] AR Holzwarth, MG Muller, G Gatzen, M Hucke, and K Griebenow. Ultrafast Spectroscopy of the Primary Electron and Energy-Transfer Processes in the Reaction-Center of Photosystem-II. Journal of Luminescence, 60-1:497-502, APR 1994. 1993 International Conference on Luminescence (ICL 93), STORRS, CT, AUG 09-13, 1993.

[36] G. Gatzen, M. G. Muller, K. Griebenow, and A. R. Holzwarth. Primary processes and structure of the photosystem ii reaction center .3. kinetic analysis of picosecond energy transfer and charge separation processes in the d1-d2-cyt-b559 complex measured by time-resolved fluorescence. Journal of Physical Chemistry, 100(17):7269-7278, April 1996.

[37] T Rech, JR Durrant, DM Joseph, J Barber, G Porter, and DR Klug. Does Slow Energy-Transfer Limit the Observed Time Constant for Radical Pair Formation in Photosystem-II Reaction Centers. Biochemistry, 33(49):14768-14774, DEC 131994.

[38] S. R. Greenfield, M. Seibert, Govindjee, and M. R. Wasielewski. Direct measurement of the effective rate constant for primary charge separation in isolated photosystem ii reaction centers. Journal of Physical Chemistry B, 101(13):22512255, March 1997.

[39] B Donovan, LA Walker, D Kaplan, M Bouvier, CF Yocum, and RJ Sension. Structure and function in the isolated reaction center complex of photosystem ii .1. ultrafast fluorescence measurements of psii. Journal of Physical Chemistry B, 101(26):5232-5238, JUN 261997. 
[40] M. L.; van Grondelle R.; Dekker J. P. \& van Stokkum I. H. M. van Mourik, F.; Groot. Global and target analysis of fluorescence measurements on photosystem 2 reaction centers upon red excitation. Physical Chemistry Chemical Physics, 6(20):4820-4824, 2004.

[41] M. L.; van Stokkum I. H. M.; Breton J. \& van Grondelle R. Pawlowicz, N. P.; Groot. Charge separation and energy transfer in the photosystem ii core complex studied by femtosecond midinfrared spectroscopy. Biophysical Journal, 93(8):2732-2742, 2007.

[42] G Hastings, JR Durrant, J Barber, G Porter, and DR Klug. Observation of Pheophytin Reduction in Photosystem 2 Reaction Centers Using Femtosecond Transient Absorption-Spectroscopy. Biochemistry, 31(33):7638-7647, AUG 25 1992.

[43] JR Durrant, G Hastings, DM Joseph, J Barber, G Porter, and DR Klug. Rate of Oxidation of P680 in Isolated Photosystem-2 Reaction Centers Monitored by Loss of Chlorophyll Stimulated-Emission. Biochemistry, 32(32):8259-8267, AUG 171993.

[44] DR Klug, T Rech, DM Joseph, J Barber, JR Durrant, and G Porter. Primary Processes in Isolated Photosystem-II Reaction Centers Probed by Magic-Angle Transient Absorption-Spectroscopy. Chemical Physics, 194(2-3):433-442, MAY 151995.

[45] L. M. C. Barter, J. R. Durrant, and D. R. Klug. A quantitative structure-function relationship for the photosystern ii reaction center: Supermolecular behavior in natural photosynthesis. Proceedings of the National Academy of Sciences of the United States of America, 100(3):946-951, 2003.

[46] G. Raszewski, W. Saenger, and T. Renger. Theory of optical spectra of photosystem ii reaction centers: Location of the triplet state and the identity of the primary electron donor. Biophysical Journal, 88(2):986-998, 2005.

[47] K. Riley, R. Jankowiak, M. Ratsep, G. J. Small, and V. Zazubovich. Evidence for highly dispersive primary charge separation kinetics and gross heterogeneity in the isolated psii reaction center of green plants. Journal of Physical Chemistry B, 108(29):10346-10356, July 2004.

[48] M. L. Groot, N. P. Pawlowicz, L. J. G. W. van Wilderen, J. Breton, I. H. M. van Stokkum, and R. van Grondelle. Initial electron donor and acceptor in isolated photosystem ii reaction centers identified with femtosecond mid-ir spectroscopy. Proceedings of the National Academy of Sciences of the United States of America, 102(37):13087-13092, 2005.

[49] B. A. Diner, E. Schlodder, P. J. Nixon, W. J. Coleman, F. Rappaport, J. Lavergne, W. F. J. Vermaas, and D. A. Chisholm. Site-directed mutations at d1-his198 and d2-his 97 of photosystem ii in synechocystis pcc 6803: Sites 
of primary charge separation and cation and triplet stabilization. Biochemistry, 40(31):9265-9281, 2001.

[50] H. Lee, Y. C. Cheng, and G. R. Fleming. Coherence dynamics in photosynthesis: Protein protection of excitonic coherence. Science, 316(5830):1462-1465, 2007.

[51] K. J. Vink, S. Deboer, J. J. Plijter, A. J. Hoff, and D. A. Wiersma. Opticaldynamics of the reaction center of photosystem-ii - a hole-burning and photonecho study. Chemical Physics Letters, 142(6):433-438, 1987. 


\section{CHAPTER V}

\section{Conclusion and Future Work}

In this thesis, I have endeavored to present a clear and concise picture of my research here at the University of Michigan. To accomplish this, I have sometimes taken the role of a spectroscopist, pushing the boundaries of current technique, and at other times have acted as biophysicist, seeking to probe the intricacies of natural photosynthesis. Both of these roles have been integral to my education and growth as a scientist.

\section{Technical Innovations}

From a technical standpoint, we have contributed several innovations which have advanced the current state of 2DES. The first two-color 2DES (2C2DES) measurement was demonstrated for energy transfer in a system of dyes connected by a short length of DNA [1]. The 2C2DES technique allows for the examination of energy transfer across large frequency separations, such that the dynamics do not show up near the diagonal of the 2D spectrum.

A significant contribution to the field has been the implementation of 2DES in the pump-probe geometry [2]. The ability to precisely control the coherence time $\left(t_{1}\right)$ with a pulse shaper and the relative phase between the first two pulses has removed the challenges of timing uncertainty and phase stability inherent to many 
other implementations, including the fully non-collinear beam geometry. While these challenges made 2DES all but inaccessible as a general-purpose spectroscopic tool to many groups, our new implementation is comparatively easier to implement. Anyone currently performing transient absorption experiments should be able to adapt their setups to 2DES with the addition of a pulse-shaper. It is our hope that this innovation will rapidly open 2DES as a tool to the larger spectroscopic community, rather than just the handful of groups who have been involved in the development of the technique. We have also demonstrated the ability to separate rephasing and non-rephasing signals via phase-cycling, a process that is often used for observing coherence dynamics [3]. In addition, we have developed phase-cycling and chopping schemes for scatter reduction, a key technical advance for studying biological samples.

Finally, we have implemented the use of a continuum probe in 2DES measurements. With this innovation, the direct and simultaneous observation of many different states and processes is possible with 2DES. For systems like the RC with multiple bands, some of which probe only a subset of chromophores, this type of detection will be invaluable. Our demonstration of this experiment on the laser dye PERY [4] allowed us not only to observe vibrational wavepacket motion on the central diagonal peak but also to directly observe cross-peaks arising from the vibronic structure of the dye, a feature that was unobserved in one-color 2DES experiments [5].

Regarding data treatment, we have developed a process for analyzing the rich kinetic information in 2DES experiments. An unconstrained fitting method maps observed time constants to the frequency-frequency space of the $2 \mathrm{D}$ spectrum to better elucidate the spectroscopic origin of the different kinetic proceses. We call these maps 2D decay associated spectra, and while they share commonalities with other fitting modalities, they represent the first method developed to specifically extract and display intuitive kinetic information from 2D spectra. Amplitude-weighted distributions of time constants additionally provide a distribution of observed decay times. 


\section{Biological Significance}

From a biological standpoint, we have applied 2DES to study a very challenging system: the D1D2-cyt.b559 reaction center complex, which is the heart of plant photosynthesis. This system has been extensively studied $[6,7,8]$, but there is still no definitive model to describe its energy and charge transfer dynamics and their relation to the RC structure. With 2DES we have produced detailed frequency-frequency correlation maps of the $\mathrm{Q}_{y}$ band with simultaneously high time and frequency resolution, providing a wealth of kinetic data that reflects the large degree of heterogeneity in the energy and charge transfer processes in the RC.

Our kinetic analysis has elucidated the origin of sub-ps spectral dynamics, an often disputed process $[9,10]$ which prior experiments have not had sufficient time and/or frequency resolution to observe/interpret. Our ability to spectrally resolve cross-peaks with 2DES has given us a great advantage in being able to understand this process. Within the first $100-200$ fs we observe the growth of energy transfer cross-peaks indicating coupling between excitons at early times, providing insight into the excitonic structure of the RC.

We have observed two distinct spectral features in our 2D decay associated spectra with time constants of 1-2 ps and 3 ps, respectively, which, with the aid of previous studies, we assign to primary charge separation. This seems to support a recent transient absorption study in which the results motivated assignments of two primary charge separation pathways [11].

We also detected another component with a time constant of $\sim 7$ ps for excitations at wavelengths shorter than $675 \mathrm{~nm}$. This is roughly consistent with a $14 \mathrm{ps}$ component observed in transient absorption studies upon excitation at $670.5 \mathrm{~nm}$ and $672 \mathrm{~nm}$ which was assigned to excitation energy transfer between pigments at $670 \mathrm{~nm}$ and $680 \mathrm{~nm}[12]$.

We observed a 40 - 60 ps component near the diagonal around $680 \mathrm{~nm}$. This time 
constant may be related to a proposed secondary charge transfer process $[11,9]$. It is also consistent with slow charge separation from a trap state nearly degenerate with the primary electron donor and reported in other $77 \mathrm{~K}$ TA studies $[11,12,13]$. The precise timing of our long-lived component could not be measured accurately due to the 150 ps maximum delay in our data but is spectrally consistent with a radical pair state.

\section{Future Work}

The 2DES studies of the D1D2-cyt.b559 reaction center complex presented here provide some answers to important questions but leave many more unanswered. We have presented a kinetic analysis of our data, interpreting the different dynamics in terms of an exciton model with the aid of many previous spectroscopic studies. Many exciton models exist for the RC, and future work aims to use 2DES to distinguish between these models. This will require simulating 2DES data starting from the excitonic Hamiltonian and including coupling to the protein bath. These efforts are currently underway. To provide the most rigorous test possible, an expanded 2DES data set is needed that draws on the technical advances presented in this thesis. Future 2DES studies will include the use of a continuum probe to monitor the pheophytin $\mathrm{Q}_{x}$ band, as well as the ion bands at $460 \mathrm{~nm}$ and 790-820 nm. Simultaneous monitoring of these bands will further clarify the kinetic contributions relating to charge separation and excitation energy transfer in regions where the spectrum is not nearly so congested as the $\mathrm{Q}_{y}$ band. A holistic treatment of the data will strengthen our ability to assign the observed kinetic terms to specific excitons or chromophores.

While we presented some preliminary data on the PSII RCs reduced with sodium dithionite prior to measurement, the continuum probe experiments will give direct confirmation that our protocol has successfully reduced the majority of complexes in the sample volume. Studies on reduced samples will allow us to remove the contri- 
butions to the data from primary charge separation and may also effect the observed excitation energy transfer events in a way that will allow us to elucidate the role of the pheophytin molecules in the various exciton states.

Additionally, polarization-dependent data will be taken to accentuate cross-peaks in the data and measure transition dipole orientations [14]. 2DES experiments on mutants may also aid in unravelling the overlapping spectral signatures of the PSII $\mathrm{RC}$.

The field of multidimensional spectroscopy continues to advance and new experiments probing higher order correlations and new frequency regimes $[15,16,17]$ continue to be proposed. In combination with better-resolved crystal structures and improved electronic structure calculations it is our hope to eventually understand the structure function relationship of the D1D2-cyt.6559 reaction center complex. 


\section{Bibliography}

[1] Kristin LM Lewis, Jeffrey A Myers, , Patrick Tekavec, and Jennifer P Ogilvie. Two-color two-dimensional fourier transform spectroscopy of energy transfer. In Ultrafast Phenomena XVI: Proceedings of the 16th International Conference, Palazzo dei Congressi Stresa, Italy, June 9-13, 2008, 2009.

[2] J. A. Myers, K. L. M. Lewis, P. F. Tekavec, and J. P. Ogilvie. Two-color twodimensional fourier transform electronic spectroscopy with a pulse-shaper. Optics Express, 16(22):17420-17428, 2008.

[3] E. Collini and G. D. Scholes. Electronic and vibrational coherences in resonance energy transfer along meh-ppv chains at room temperature. Journal of Physical Chemistry A, 113(16):4223-4241, 2009.

[4] P. E. Tekavec, J. A. Myers, K. L. M. Lewis, and J. P. Ogilvie. Two-dimensional electronic spectroscopy with a continuum probe. Optics Letters, 34(9):1390-1392, 2009 .

[5] A. Nemeth, F. Milota, T. Mancal, V. Lukes, J. Hauer, H. F. Kauffmann, and J. Sperling. Vibrational wave packet induced oscillations in two-dimensional electronic spectra. i. experiments. Journal of Chemical Physics, 132(18):184514, May 2010.

[6] SR Greenfield and MR Wasielewski. Excitation energy transfer and charge separation in the isolated photosystem ii reaction center. Photosynthesis Research, 48(1-2):83-97, MAY 1996.

[7] LM Yoder, AG Cole, and RJ Sension. Structure and function in the isolated reaction center complex of photosystem ii: energy and charge transfer dynamics and mechanism. Photosynthesis Research, 72(2):147-158, 2002.

[8] J. P. Dekker and R. van Grondelle. Primary charge separation in photosystem ii. Photosynthesis Research, 63(3):195-208, 2000.

[9] V. I. Prokhorenko and A. R. Holzwarth. Primary processes and structure of the photosystem ii reaction center: A photon echo study. Journal of Physical Chemistry B, 104(48):11563-11578, 2000. 
[10] V. Zazubovich, R. Jankowiak, K. Riley, R. Picorel, M. Seibert, and G. J. Small. How fast is excitation energy transfer in the photosystem ii reaction center in the low temperature limit? hole burning vs photon echo. Journal of Physical Chemistry B, 107(12):2862-2866, 2003.

[11] E. Romero, I. H. M. van Stokkum, V. I. Novoderezhkin, J. P. Dekker, and R. van Grondelle. Two different charge separation pathways in photosystem ii. Biochemistry, 49(20):4300-4307, 2010.

[12] HM VIisser, ML Groot, F van Mourik, IHM van Stokkum, JP Dekker, and R van Grondelle. Subpicosecond Transient Absorption Difference Spectroscopy on the Reaction-Center of Photosystem-II - Radical Pair Formation at 77-K. Journal of Physical Chemistry, 99(41):15304-15309, OCT 121995.

[13] M. L. Groot, F. vanMourik, C. Eijckelhoff, I. H. M. vanStokkum, J. P. Dekker, and $\mathrm{R}$. vanGrondelle. Charge separation in the reaction center of photosystem ii studied as a function of temperature. Proceedings of the National Academy of Sciences of the United States of America, 94(9):4389-4394, April 1997.

[14] E. L. Read, G. S. Engel, T. R. Calhoun, T. Mancal, T. K. Ahn, R. E. Blankenship, and G. R. Fleming. Cross-peak-specific two-dimensional electronic spectroscopy. Proceedings of the National Academy of Sciences of the United States of America, 104(36):14203-14208, 2007.

[15] J. P. Ogilvie and K. J. Kubarych. Multidimensional electronic and vibrational spectroscopy: An ultrafast probe of molecular relaxation and reaction dynamics. Advances In Atomic, Molecular, and Optical Physics, Vol 57, 57:249-321, 2009.

[16] W. Zhuang, T. Hayashi, and S. Mukamel. Coherent multidimensional vibrational spectroscopy of biomolecules: Concepts, simulations, and challenges. Angewandte Chemie-international Edition, 48(21):3750-3781, 2009.

[17] M. H. Cho. Coherent two-dimensional optical spectroscopy. Chemical Reviews, 108(4):1331-1418, 2008. 
APPENDICES 


\section{APPENDIX A}

\section{Preliminary Data on Reduced RC Samples}

\section{Sodium Dithionite Treatment}

The spectral congestion of the $\mathrm{Q}_{y}$ band of the reaction center particles makes interpretation of the kinetics difficult even with the high resolution and increased dimensionality inherent to 2DES experiments. In order to help separate the contributions to the kinetics from charge separation and other processes such as excitation energy transfer, data were taken on regular samples and samples in which charge separation was prevented by treatment with the chemical sodium dithionite.

In the dark, sodium dithionite is known to reduce the cytochrome $b-559$ of the RC. Under actinic light, charge separation occurs, and the D1 pheophytin becomes trapped in its anionic state, while the electron donor $\left(\mathrm{P}_{680^{+}}\right)$is reduced back to its neutral state either directly by the dithionite or indirectly, via dithionite-mediated action by the cytochrome or the $\mathrm{D} 1$ tyrosine $161\left(\mathrm{Y}_{Z}\right)[1]$. The charge state of the pheophytin can be measured in the linear spectrum by observing a bleach in the pheophytin $\mathrm{Q}_{x}$ band near 544-545 $\mathrm{nm}$. Pre-reduction of the active pheophytin prevents charge separation from occurring following the photoexcitation of P680, thus removing one or more decay pathways from the spectrally degenerate kinetics near 
$680 \mathrm{~nm}$. This technique has been frequently utilized in several previous spectroscopic studies on this sample $[2,3,4,5,6,7,8]$.

Our protocol for the dithionite treatment is adapted from that of Jankowiak, et.al. [9]. An atmosphere of dry nitrogen was established in a glove bag, containing the RC sample, a volume of low salt BTS-200 buffer, and glycerol. The glycerol was bubbled with nitrogen for 24 hours prior to use to ensure removal of oxygen from the viscous cryoprotectant. The buffer was exposed to the nitrogen atmosphere in a petri dish, allowing the gas to flow over a high surface area, for several hours before use. $62.5 \mathrm{mg}$ of solid sodium dithionite was added to $0.5 \mathrm{~mL}$ of buffer and mixed until fully dissolved. $2.5 \mu \mathrm{L}$ of dithionite solution was added with $35 \mu \mathrm{L}$ of glycerol to 15 $\mu \mathrm{L}$ of sample solution and homogenized thoroughly with a vortex mixer. The final concentration of sodium dithionite in the sample was $6 \mathrm{mg} / \mathrm{mL}$. The volumetric ratio of glycerol to aqueous buffer was maintained at 2:1 as in the untreated samples.

The sample solution was then loaded under nitrogen into an airtight sample cell described previously. To activate the dithionite, the sample cell was illuminated through $2 \mathrm{~cm}$ water with actinic white light from a tungsten source for 30 minutes. After illumination, a linear spectrum of the pheophytin $\mathrm{Q}_{x}$ band confirmed that the treatment was successful. Samples were then immediately placed in the cryostat and rapidly cooled to a temperature of $77 \mathrm{~K}$ in preparation for the 2DES experiment.

\section{Reduced PSII Studies}

Figures A.1 - A.3 show examples of $2 \mathrm{D}$ spectra for several $t_{2}$ values in the reduced data. 2D decay associated spectra were calculated with the same fitting algorithm and clustering procedure as in the native reaction centers. Figure A.4 shows the resulting 2D DAS for the reduced data. 


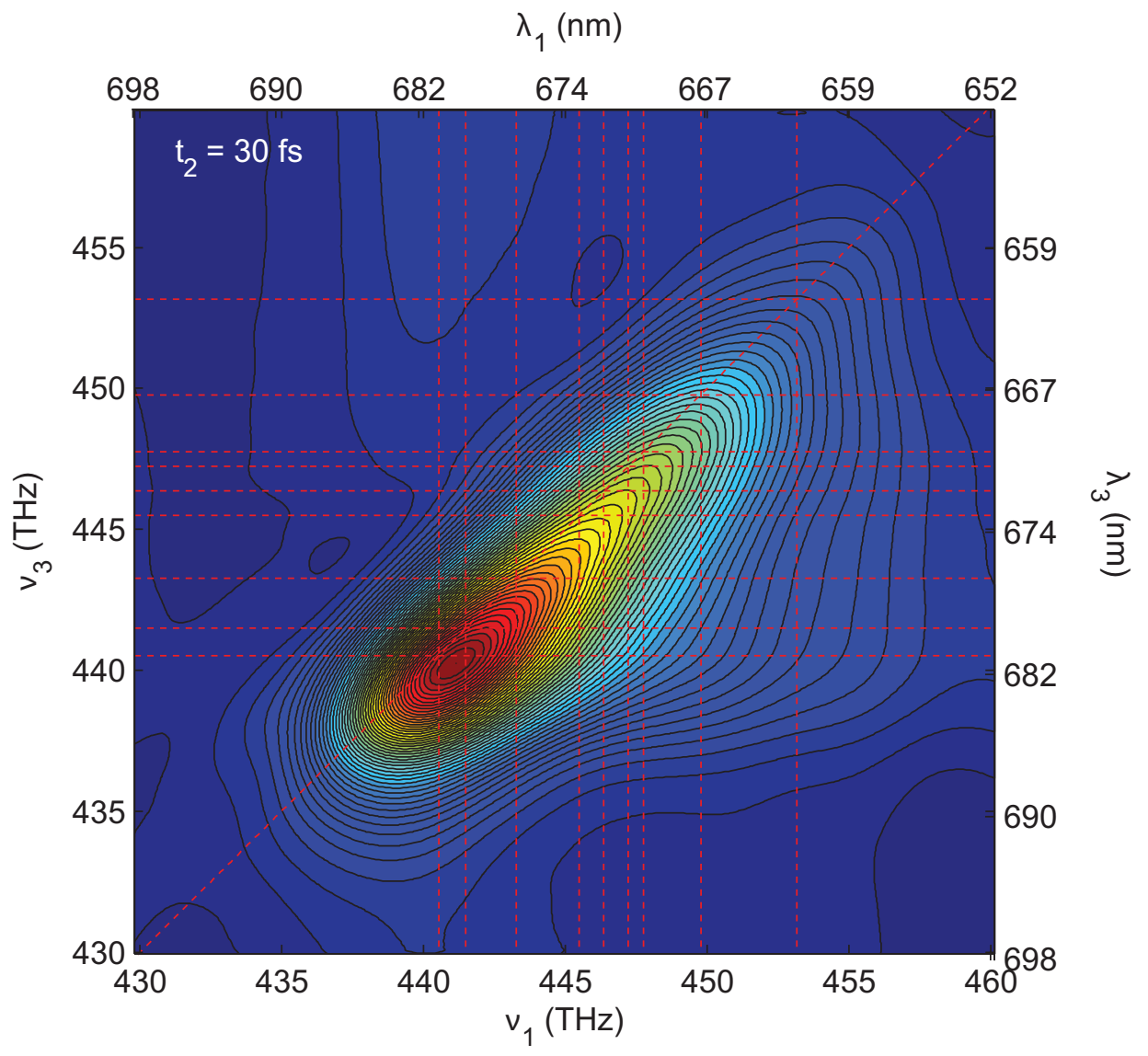

Figure A.1: 2D Data on the reduced D1D2-cyt.b559 reaction center at $77 \mathrm{~K}$ at $t_{2}=$ $30 \mathrm{fs}$ 

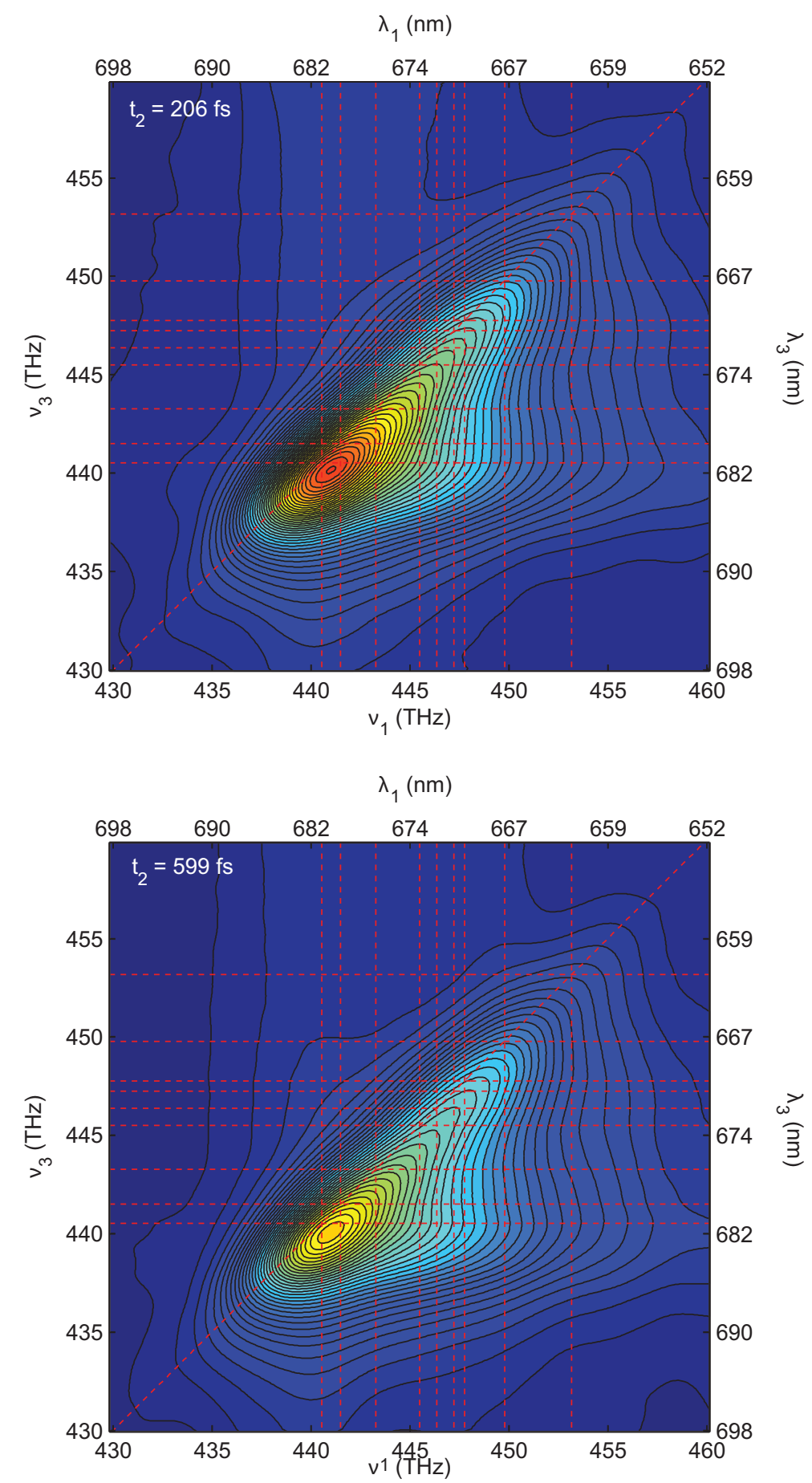

Figure A.2: 2D Data on the reduced D1D2-cyt.6559 reaction center at $77 \mathrm{~K}$ at $t_{2}=$ $206 \mathrm{fs}$ (top) and $t_{2}=599 \mathrm{fs}$ (bottom) 

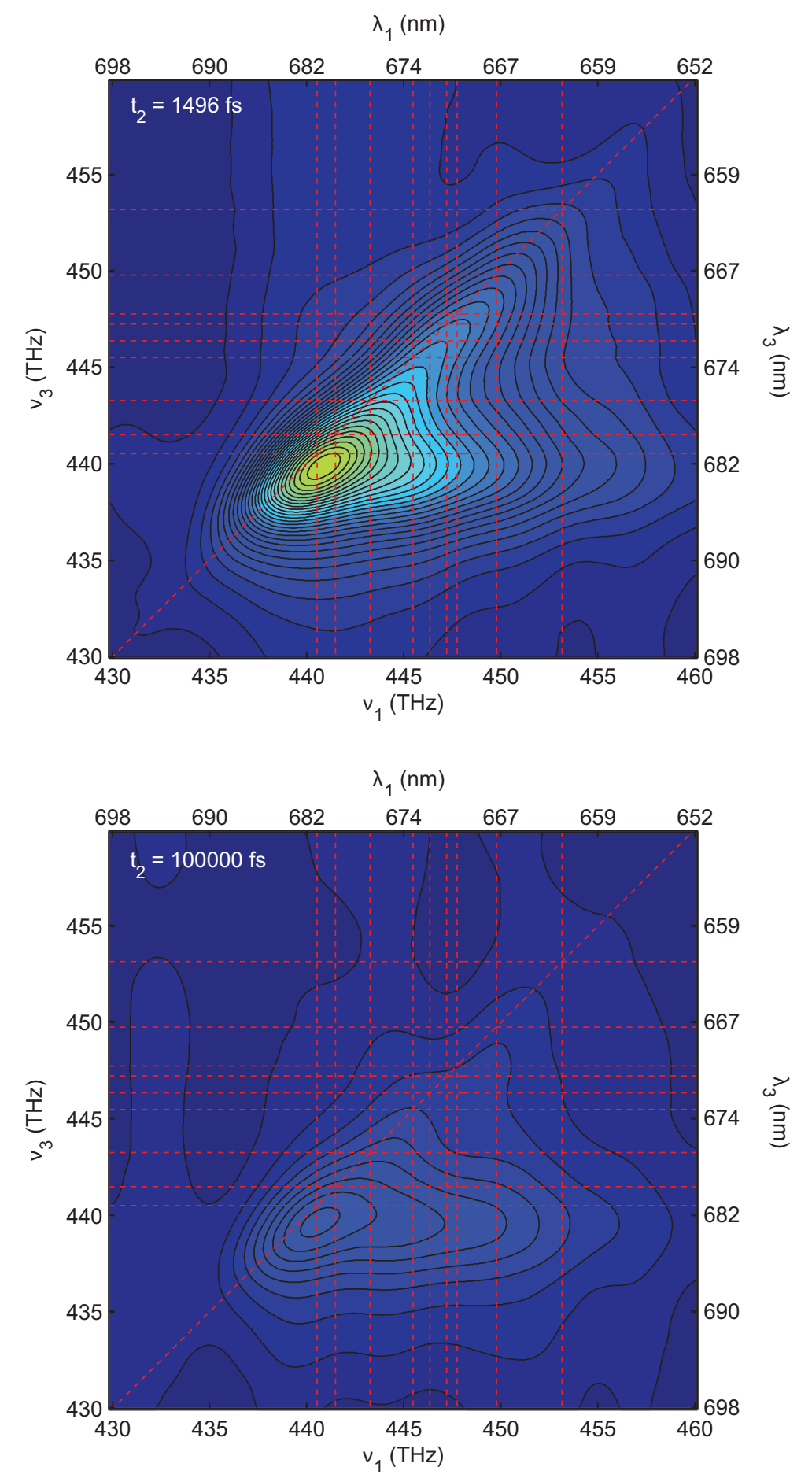

Figure A.3: 2D Data on the reduced D1D2-cyt. 6559 reaction center at $77 \mathrm{~K}$ at $t_{2}=$ $1.5 \mathrm{ps}$ (top) and $t_{2}=100 \mathrm{ps}$ (bottom) 

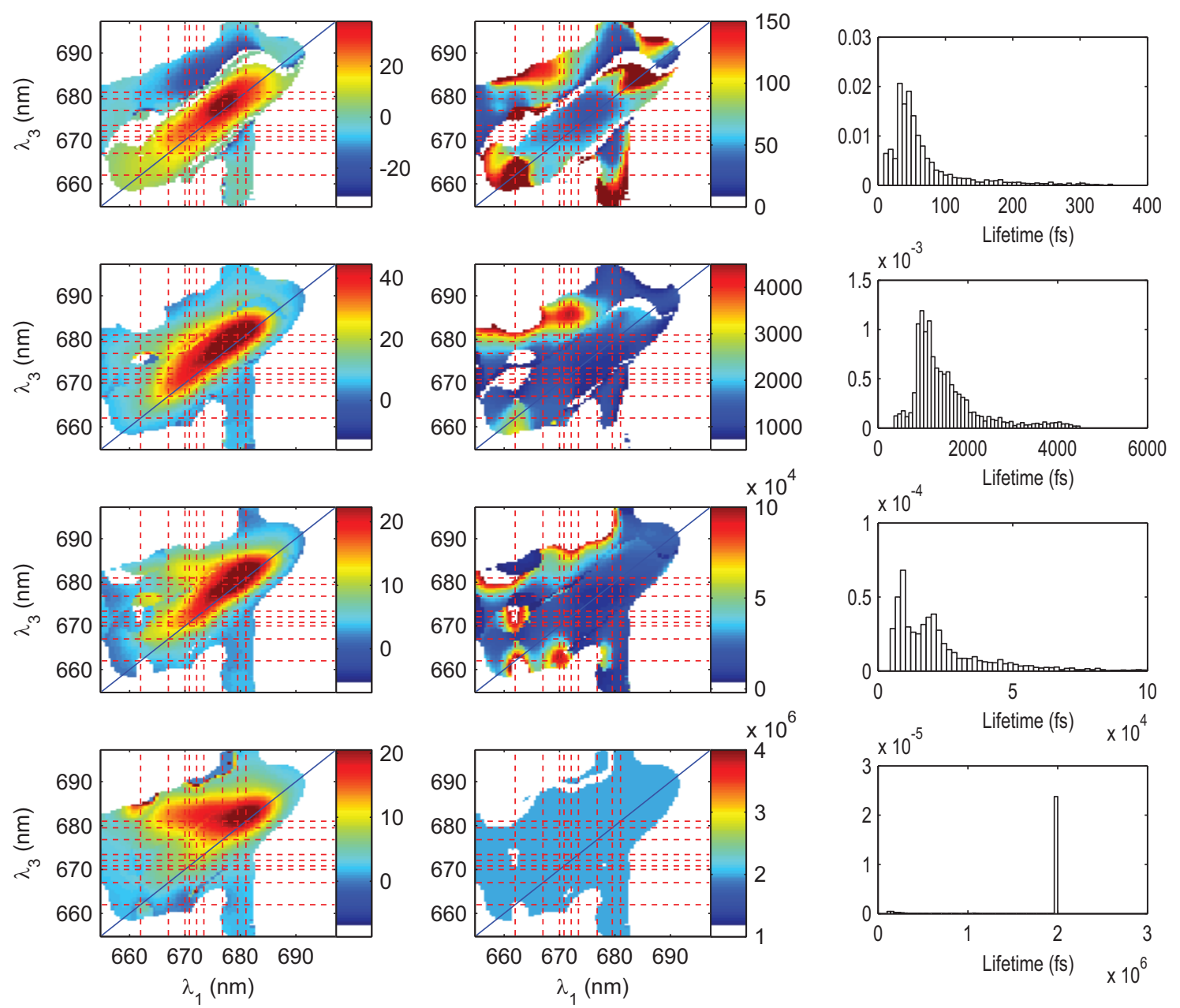

Figure A.4: 2D DAS spectra in the reduced D1D2-cyt.6559 reaction center at $77 \mathrm{~K}$. 


\section{Observations}

In the reduced data, the first $2 \mathrm{D}$ DAS component (I) exhibits the same rapid energy equilibration in $\mathrm{CP} \# 1$ as in the native RC, though the transfer between at least some of the exciton states involved seems to be slower, $\sim 100-150$ fs. This indicates that sub-ps energy transfer still occurs in these samples, but the reduction of the pheophytin molecules may affect the rates.

In component II, the diagonal feature is more inhomogeneously broadened. We note that the cross-peak with the $\sim 3$ ps feature is still present, though its rate has slowed to a time of roughly 4 ps. This provides evidence against the interpretation of this element as the 3 ps charge separation pathway cited in Romero at al. [10], as they have assigned $\mathrm{Pheo}_{D 1}$ as the electron acceptor for that charge separation pathway. The diagonal peak in this component, however, has its lifetime distribution shifted dramatically toward 1 ps. While the cross-peak feature may be due to some other process, the change in lifetime around the diagonal is consistent with a 1 ps charge separation process involving only chlorophyll molecules: $\mathrm{P}_{D 1} \mathrm{P}_{D 2} \mathrm{Chl}_{D 1}^{*} \longrightarrow$ $\mathrm{P}_{D 1}^{+} \mathrm{Chl}_{D 1}^{-}$, consistent with Romero et al. [10]. The shift of the distribution toward 2 ps in the native RC may have been evidence of spectrally degenerate contributions from both charge separation pathways, one of which $\left(\mathrm{Chl}_{D 1} \mathrm{Pheo}_{D 1}^{*} \longrightarrow \mathrm{Chl}_{D 1}^{+} \mathrm{Pheo}_{D 1}^{-}\right)$ becomes disabled following dithionite treatments.

The 2D DAS component III displays a far more heterogeneous character in the reduced $\mathrm{RC}$ than in the native sample, and the associated lifetime of all the features is now shifted toward 5-15 ps, somewhat consistent with the diagonal feature near 670 $\mathrm{nm}$ in the native RC. There has been a sharp shift in distribution near $680 \mathrm{~nm}$, such that a component of $\sim 40-60 \mathrm{ps}$ is no longer observed here. This is consistent with the loss of trap state or secondary charge transfer found at this wavelength in transient absorption studies $[11,12,10]$, strengthening our interpretation of this component.

The final 2D DAS component looks similar for the reduced and native samples. 
However, the final component in the reduced data resembles the spectrum of $\mathrm{P}_{D 1}^{+}$ $\mathrm{Chl}_{D 1}^{-}$reported by Romero et al. [10], which is consistent with the interpretation that the rapid (1 ps) charge transfer pathway is active up to the step involving the trap state in these samples.

We note that the reduced data here are only preliminary. Further studies will use a continuum probe pulse to simultaneously observe the pheophytin $\mathrm{Q}_{x}$ band, offering confirmation that the samples have been properly reduced and remain in that state throughout the experiment. 


\section{Bibliography}

[1] SR Greenfield and MR Wasielewski. Excitation energy transfer and charge separation in the isolated photosystem ii reaction center. Photosynthesis Research, 48(1-2):83-97, MAY 1996.

[2] MR Wasielewski, DG Johnson, Govindjee, C Preston, and M Seibert. Determination of the Primary Charge Separation Rate in Photosystem-II Reaction Centers at 15-K. Photosynthesis Research, 22(1):89-99, OCT 1989.

[3] MR Wasielewski, DG Johnson, M Seibert, and Govindjee. Determination of the Primary Charge Separation Rate in Isolated Photosystem-II Reaction Centers with 500-fs Time Resolution. Proceedings of the National Academy of Sciences of the United States of America, 86(2):524-528, JAN 1989.

[4] G. P. Wiederrecht, M. Seibert, Govindjee, and M. R. Wasielewski. Femtosecond photodichroism studies of isolated photosystem-ii reaction centers. Proceedings of the National Academy of Sciences of the United States of America, 91(19):89999003, 1994.

[5] R Jankowiak, D Tang, GJ Small, and M Seibert. Transient and persistent hole burning of the reaction center of photosystem-ii. Journal of Physical Chemistry, 93(4):1649-1654, FEB 231989.

[6] D. Tang, R. Jankowiak, M. Seibert, C. F. Yocum, and G. J. Small. Excited-state structure and energy-transfer dynamics of 2 different preparations of the reaction center of photosystem-ii - a hole-burning study. Journal of Physical Chemistry, 94(17):6519-6522, August 1990.

[7] V. L. Tetenkin, B. A. Gulyaev, M. Seibert, and A. B. Rubin. Spectral properties of stabilized $\mathrm{d} 1 / \mathrm{d} 2 /$ cytochrome-b-559 photosystem-ii reaction center complex effects of triton x-100, the redox state of pheophytin, and beta-carotene. Febs Letters, 250(2):459-463, July 1989.

[8] J Breton. Orientation of the pheophytin primary electron acceptor and of the cytochrome b559 in the $\mathrm{d} 1 \mathrm{~d} 2$ photosystem ii reaction center. In Perspectives in Photosynthesis: proceedings of the Twenty second Jerusalem Symposium on Quantum Chemistry and Biochemistry, 1989. 
[9] R. Jankowiak, M. Ratsep, R. Picorel, M. Seibert, and G. J. Small. Excited states of the 5-chlorophyll photosystem ii reaction center. Journal of Physical Chemistry B, 103(44):9759-9769, November 1999.

[10] E. Romero, I. H. M. van Stokkum, V. I. Novoderezhkin, J. P. Dekker, and R. van Grondelle. Two different charge separation pathways in photosystem ii. Biochemistry, 49(20):4300-4307, 2010.

[11] HM VIisser, ML Groot, F van Mourik, IHM van Stokkum, JP Dekker, and R van Grondelle. Subpicosecond Transient Absorption Difference Spectroscopy on the Reaction-Center of Photosystem-II - Radical Pair Formation at 77-K. Journal of Physical Chemistry, 99(41):15304-15309, OCT 121995.

[12] M. L. Groot, F. vanMourik, C. Eijckelhoff, I. H. M. vanStokkum, J. P. Dekker, and R. vanGrondelle. Charge separation in the reaction center of photosystem ii studied as a function of temperature. Proceedings of the National Academy of Sciences of the United States of America, 94(9):4389-4394, April 1997. 


\section{APPENDIX B}

\section{Electronic Coherences}

Researchers using similar 2D techniques have recently found evidence of longlasting coherences between exciton states in the sample which persist during the population time $[1,2,3,4,5]$. It has been suggested that photosynthetic systems may be able to sample several energy pathways quantum mechanically before "choosing" the most efficient route [6].

To search for coherences in our data, we examined the residuals of the exponential fits to the absorptive spectra. Coherences should appear as oscillatory features in the data which would be accentuated following a subtraction of the dominant exponential decay terms. This same approach was used by Engel et al. to isolate the coherences in the Fenna-Matthews-Olson bacterial antenna complex [2]. The residuals form a $3 \mathrm{D}$ matrix $r\left(\nu_{1}, \nu_{3}, t_{2}\right)$.

As Collini et al. observed [5], for a true electronic coherence, there should be oscillations present for cross-peaks both above and below the diagonal, and these should be out of phase with one another. To identify cross-peaks exhibiting this feature, I produced a covariance matrix $C\left(\nu_{1}, \nu_{3}\right)$, where each coordinate gives the covariance between the upper and lower cross-peak at coordinates $\left(\nu_{1}, \nu_{3}\right)$ and $\left(\nu_{3}, \nu_{1}\right)$, respectively. The covariance plots were expected to be most illuminating for early 


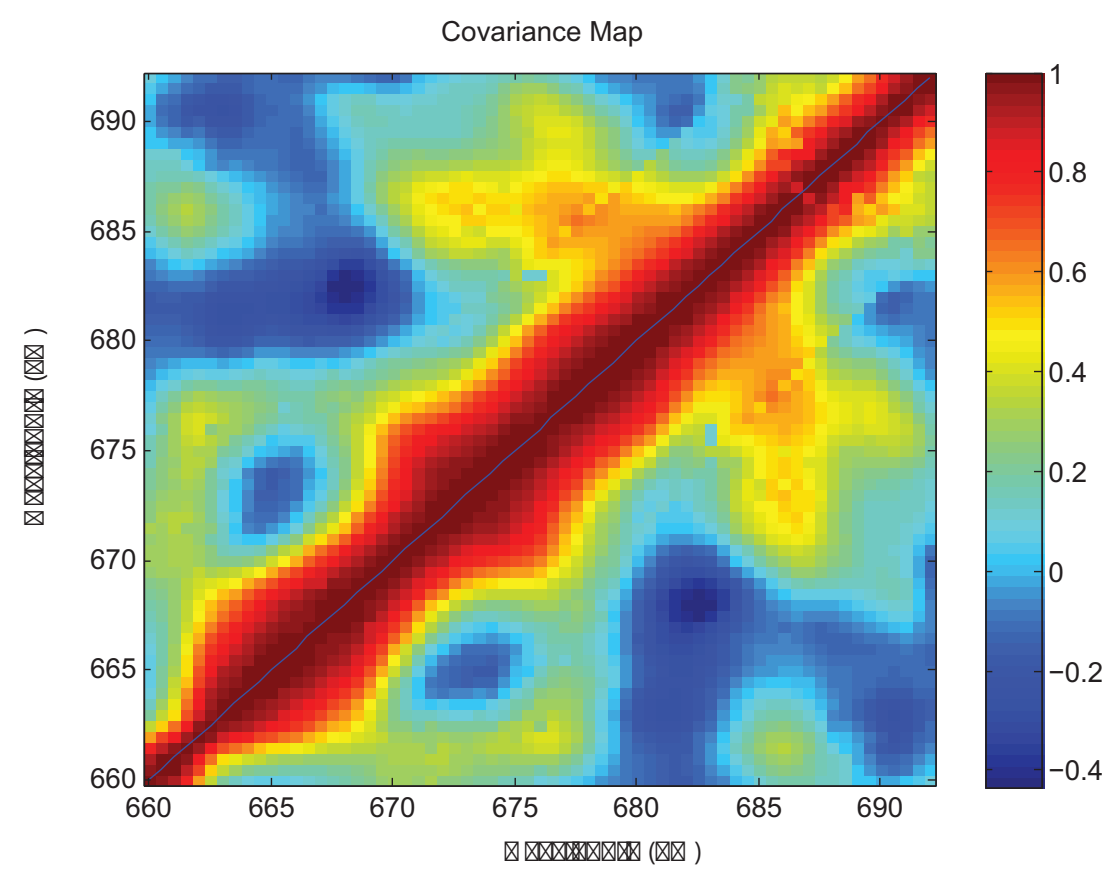

Figure B.1: Covariance map between the upper and lower cross peaks located at each point and its conjugate across the diagonal.

population times $(\sim 30-850 \mathrm{fs})$ but were examined for several different temporal windows. Figure B.1 shows a covariance plot for our residuals matrix. The areas of interest are the regions with a large negative covariance, indicating a strong anticorrelation between the upper and lower diagonals at these frequencies. The residuals in the region of strong anti-correlation at $(668 \mathrm{~nm}, 682.5 \mathrm{~nm})$ are plotted against each other in Figure B.2.

As stated in [2], the frequency of the coherence oscillations should correspond to the difference frequency between the coupled excitons. We performed a spectral analysis on the oscillations shown above, but there was very little amplitude to support a frequency component between excitons at the location of the cross-peak. A strong peak was expected at the difference frequency of $320 \mathrm{~cm}^{-1}$, but the dominant frequency component was observed at $160 \mathrm{~cm}^{-1}$. Indeed, a comparison of the oscillation frequency spectrum to the exciton levels mentioned above from Novoderezhkin et al. 
Anticorrelation between the upper and lower diagonal cross peaks

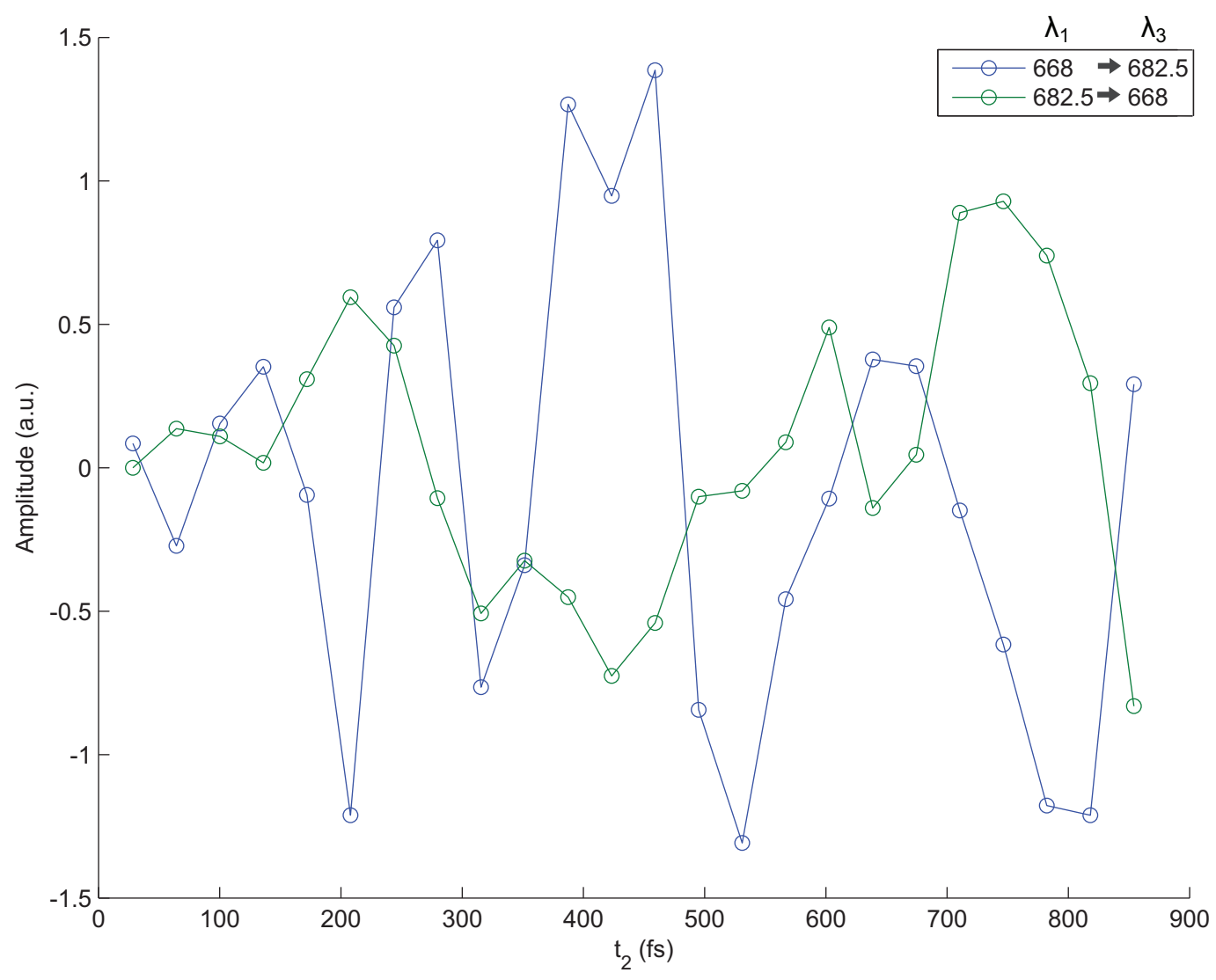

Figure B.2: Coherence plot of the residuals from RC data for the upper diagonal (blue) and lower diagonal (green) cross peaks corresponding to potential coupling between excitons at $668 \mathrm{~nm}$ and $682.5 \mathrm{~nm}$. 
[7] did not show any strong correspondence. The measured $160 \mathrm{~cm}^{-1}$ peak corresponds to a minimum in the spectrum of exciton difference frequencies. Therefore, it is likely that the residual oscillation was due to some source other than an electronic coherence. We believe that our lack of observation of electronic coherence states may reflect the smaller excitonic coupling and larger static disorder in the PSII reaction center, compared with the other systems cited above.

It has also been suggested that the observed coherences may be vibrational rather than electronic in nature [8]. The photosynthetic complexes show resonance Raman spectra that contain many low frequency modes within the same range of typical exciton difference frequencies $[9,10]$. As seen in our PERY experiments, vibrational wavepacket motion alters the lineshapes and peak amplitudes in 2DES spectra. Theoretical work by the Fleming group suggests that electronic coherences have distinct signatures such as anti-correlated width and peak amplitudes [11]. However, this work did not include possible vibrational effects. Further experimental and theoretical work is needed to confirm the electronic nature of the observed coherences and to determine their biological significance. 


\section{Bibliography}

[1] A. V. Pisliakov, T. Mancal, and G. R. Fleming. Two-dimensional optical threepulse photon echo spectroscopy. ii. signatures of coherent electronic motion and exciton population transfer in dimer two-dimensional spectra. Journal of Chemical Physics, 124, 2006.

[2] G. S. Engel, T. R. Calhoun, E. L. Read, T. K. Ahn, T. Mancal, Y. C. Cheng, R. E. Blankenship, and G. R. Fleming. Evidence for wavelike energy transfer through quantum coherence in photosynthetic systems. Nature, 446(7137):782$786,2007$.

[3] H. Lee, Y. C. Cheng, and G. R. Fleming. Coherence dynamics in photosynthesis: Protein protection of excitonic coherence. Science, 316(5830):1462-1465, 2007.

[4] E. Collini and G. D. Scholes. Electronic and vibrational coherences in resonance energy transfer along meh-ppv chains at room temperature. Journal of Physical Chemistry A, 113(16):4223-4241, 2009.

[5] E. Collini, C. Y. Wong, K. E. Wilk, P. M. G. Curmi, P. Brumer, and G. D. Scholes. Coherently wired light-harvesting in photosynthetic marine algae at ambient temperature. Nature, 463(7281):644-U69, 2010.

[6] R. J. Sension. Biophysics - quantum path to photosynthesis. Nature, 446(7137):740-741, April 2007.

[7] V. I. Novoderezhkin, J. P. Dekker, and R. van Grondelle. Mixing of exciton and charge-transfer states in photosystem ii reaction centers: Modeling of stark spectra with modified redfield theory. Biophysical Journal, 93:1293-1311, 2007.

[8] L. Z. Sharp, D. Egorova, and W. Domcke. Efficient and accurate simulations of two-dimensional electronic photon-echo signals: Illustration for a simple model of the fenna-matthews-olson complex. Journal of Chemical Physics, 132(1), 2010.

[9] J. R. Diers and D. F. Bocian. Q(y)-excitation resonance raman-spectra of bacteriochlorophyll observed under fluorescence-free conditions - implications for cofactor structure in photosynthetic proteins. Journal of the American Chemical Society, 117(24):6629-6630, June 1995. 
[10] V. Zazubovich, I. Tibe, and G. J. Small. Bacteriochlorophyll a franck-condon factors for the $\mathrm{s}-0-i \mathrm{~s}-1(\mathrm{q}(\mathrm{y}))$ transition. Journal of Physical Chemistry B, 105(49):12410-12417, December 2001.

[11] Y. C. Cheng, G. S. Engel, and G. R. Fleming. Elucidation of population and coherence dynamics using cross-peaks in two-dimensional electronic spectroscopy. Chemical Physics, 341(1-3):285-295, 2007. 


\section{APPENDIX C}

\section{ZAP-SPIDER}

In order to obtain the highest time and frequency resolution and to avoid complications in analysis that arise from having heavily chirped pulses, it is necessary to characterize and compress the pump and probe pulses to near the transform limit. This can generally be done with many different nonlinear methods, but in our case it is valuable to not only be able to measure the pulse duration but to also recover the spectral phase.

Zero Additional Phase Spectral Phase Interferometry for Direct Electric-field Reconstruction (ZAP-SPIDER) is a method developed by Riedle and coworkers [1] for single-shot full pulse characterization. It differs from the SPIDER technique of Walmsley [2] in that the test pulse only hits reflective optics so as not to add additional dispersion to the reconstructed pulse. In this implementation, a single copy of the test pulse is used with two copies of the chirped pulse to produce the measured signals. The setup is shown in Figure C.1.

An $800 \mathrm{~nm}, \sim 25 \mu \mathrm{J}$ pulse is taken as a $4 \%$ reflection from a cover slide between the first and second NOPA. This beam passes through $14 \mathrm{~cm}$ of SF-11 glass, which provides a second order material dispersion of $25,000 \mathrm{fs}^{2}$, chirping the pulse to a duration 3 ps. A 50/50 beam splitter creates two replicas of the chirped pulses, 


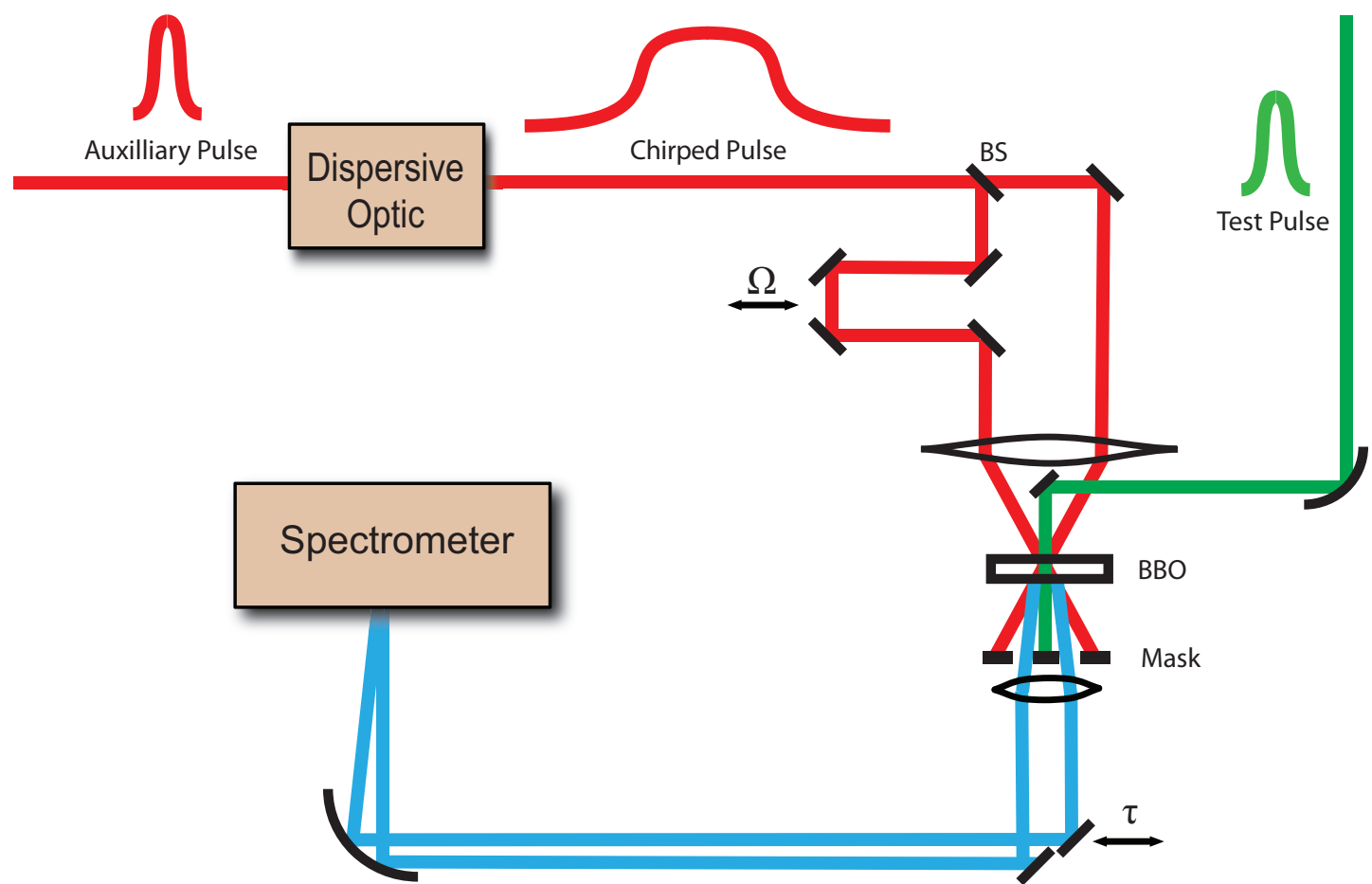

Figure C.1: A diagram of the experimental setup for ZAP-SPIDER pulse characterization. The auxiliary pulse is a $4 \%$ reflection of our regen output, and the test pulse can be any pulse which interacts with our samples.

one of which hits an adjustable interferometer arm. the two chirped pulses then are directed onto a large diameter lens, parallel to one another and in the same horizontal plane so that they will converge at the focal point of the lens. The test pulse is focused onto the same spot with the aid of a curved mirror and a small pick-off mirror placed in front of the lens. The geometry is shown in Figure C.1.

A $25 \mu \mathrm{m}$ BBO crystal is positioned at the common beam focus and aligned for sumfrequency generation (SFG) between the test pulse and each of the two chirped pulses. For visible test pulses, the SFG signals will have frequencies in the UV. Assuming a test pulse of significantly shorter duration that the chirped pulses, the test pulse can be considered to be frequency up-converted monochromatically, mixing only with the frequency component of the chirped pulse which is temporally overlapped with the test pulse in the BBO. By delaying the chirped pulses relative to one another, each signal pulse will be mixed with a different frequency, thus introducing a spectral 


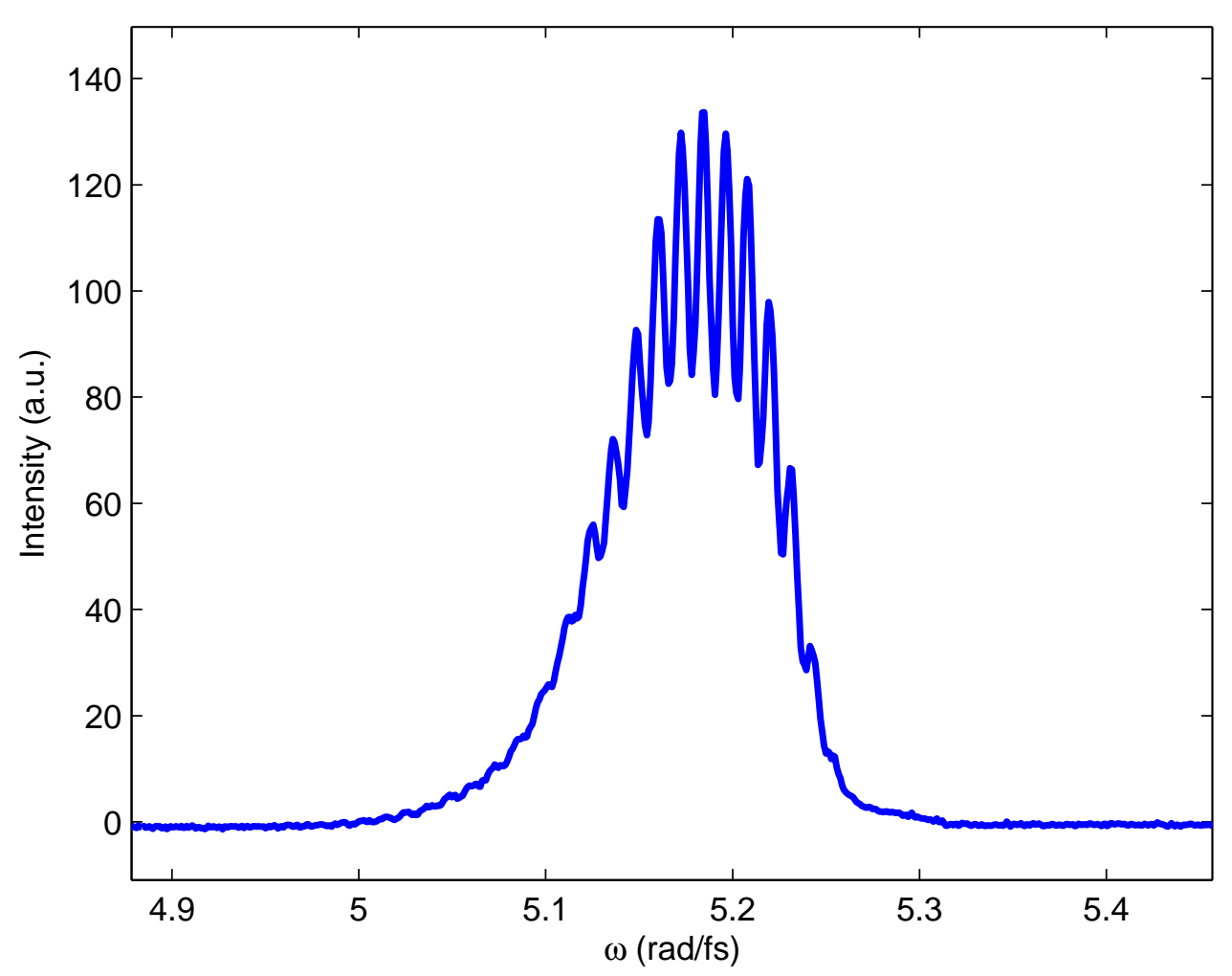

Figure C.2: A ZAP-SPIDER interferogram. Noise is suppressed by averaging over several hundred laser shots.

shear $(\Omega)$ between the signal pulses. The two signals are collimated and isolated with a spatial mask. Another time delay $(\tau)$ is introduced between the signal pulses, which are combined interferometrically in the spectrometer. A sample ZAP-SPIDER interferogram is shown in Figure C.2.

Though the ZAP-SPIDER is a single shot method, a calibration scan must be taken to remove the phase contribution due to the time delay $\tau$. In order to do this, the pulse shear is set to $\Omega=0$. The chirped pulse interferometer micrometer position corresponding to zero shear can easily be calibrated by replacing the crystal with a $10 \mu \mathrm{m}$ pinhole and measuring the spectral interferogram between the chirped pulses on the spectrometer. The exact value of non-zero shear as a function of micrometer position cannot be determined from time delay measured in the chirped pulse 
interferogram without taking into account higher orders of dispersion from the SF-11 block. It is far easier to observe the shear as the frequency shift in the SFG signal spectral envelope when the micrometer is adjusted away from the zero-shear value. The value of $\Omega$ determines the spectral resolution of the retrieved phase, and errors in $\Omega$ have a multiplicative effect on the retrieved phase coefficients, so it is advisable to choose a value for $\Omega$ equal to roughly $5-10 \%$ of the signal pulse bandwidth.

Analysis of the ZAP-SPIDER trace is performed with a concatenative algorithm developed by Walmsley and coworkers [2]. The interferogram is inverse Fourier transformed into the time domain $(\tau)$. Following the general principle of spectral interferometry, a supergaussian window is used to select the well-separated peak corresponding to a positive $\tau$ delay [3]. Fourier transformation into the frequency domain then yields a complex field from which a phase $\theta(\omega)$ is extracted. The phase of the calibration interferogram is subtracted from this in order to remove the delay term $\omega \tau$ introduced by the delay between signals. The remaining phase $\theta(\omega)$ contains contributions only from the test pulse phase and the spectral shear. The relationship between the measured phase $\theta(\omega)$ and the test pulse phase $\phi(\omega)$ is given by

$$
\theta\left(\omega_{c}\right)=\phi\left(\omega_{c}\right)-\phi\left(\omega_{c}-\Omega\right)
$$

for some center frequency $\omega_{c}$. To retrieve the test pulse phase, we sample the measured phase in intervals of $\Omega$ from $\omega_{c}$. For the sake of simplicity, we remove an unknown constant phase term $\theta_{0}$ from all phase values to eliminate a linear term that would otherwise appear in the reconstructed phase. The phase at some frequency $\omega_{0}$ is set to $\phi\left(\omega_{0}\right)=0$ so as to give a starting point $\phi\left(\omega_{0}-\Omega\right)=-\theta\left(\omega_{0}\right)$ for the reconstruction algorithm. The rest of the phase, sampled in invervals of the shear value, is reconstructed with the following equations. 


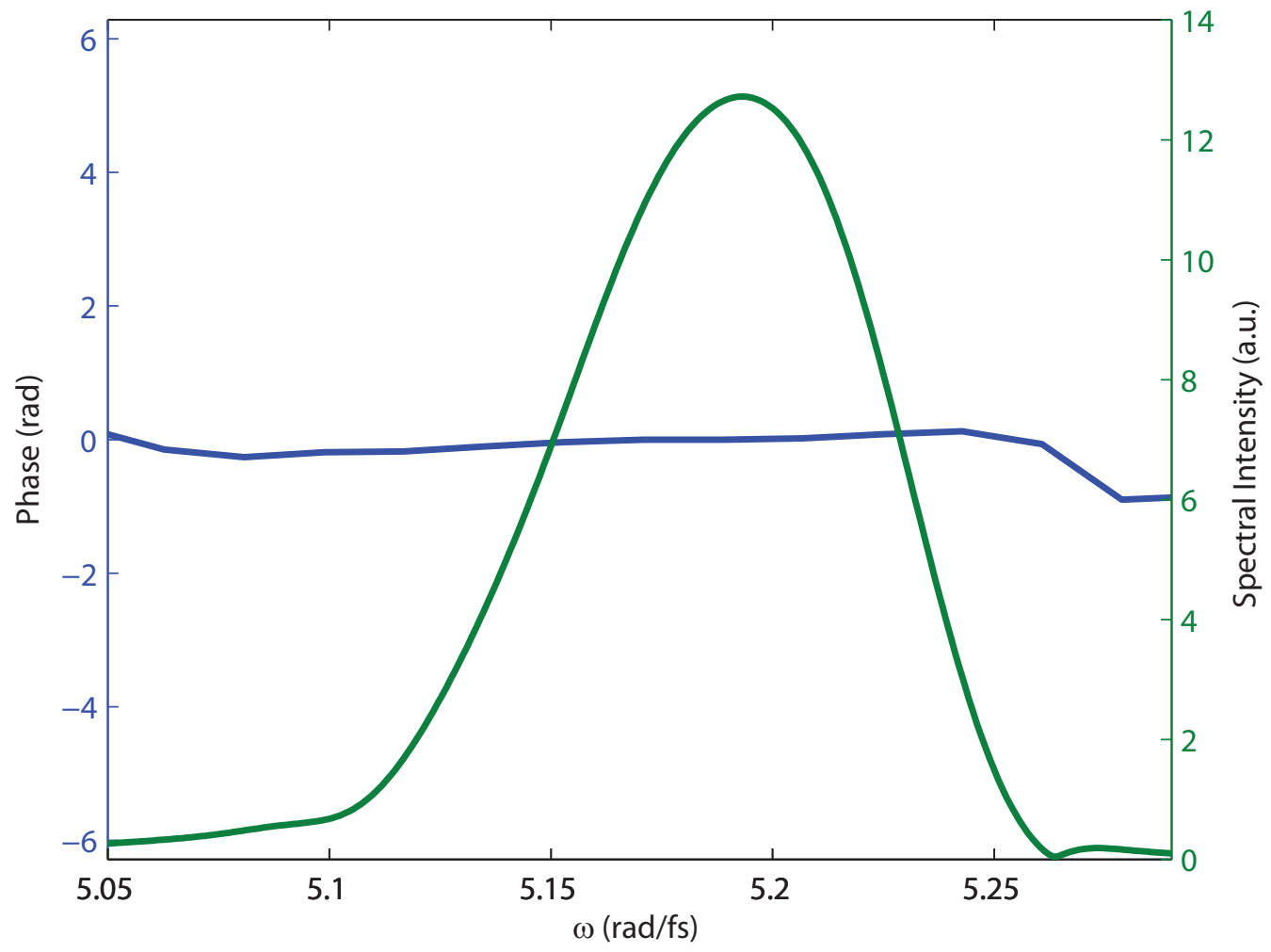

Figure C.3: A retrieved spectrum (blue line) and phase (green line) of a nearly transform limited pulse (solid line) with a FWHM of $\sim 28$ fs.

$$
\begin{aligned}
\phi\left(\omega_{0}-N \Omega\right) & =-\sum_{j=1}^{N} \theta\left(\omega_{0}+(j-1) \Omega\right) \\
\phi\left(\omega_{0}\right) & =0 \\
\phi\left(\omega_{0}+N \Omega\right) & =\sum_{j=1}^{N} \theta\left(\omega_{0}+j \Omega\right)
\end{aligned}
$$

The retrieved amplitude and phase for a nearly transform limited pulse with subradian phase variance are shown in Figure C.3. After the phase is reconstructed, an inverse Fourier transform can be used to view the temporal profile of the test pulse, as shown in Figure C.4. 


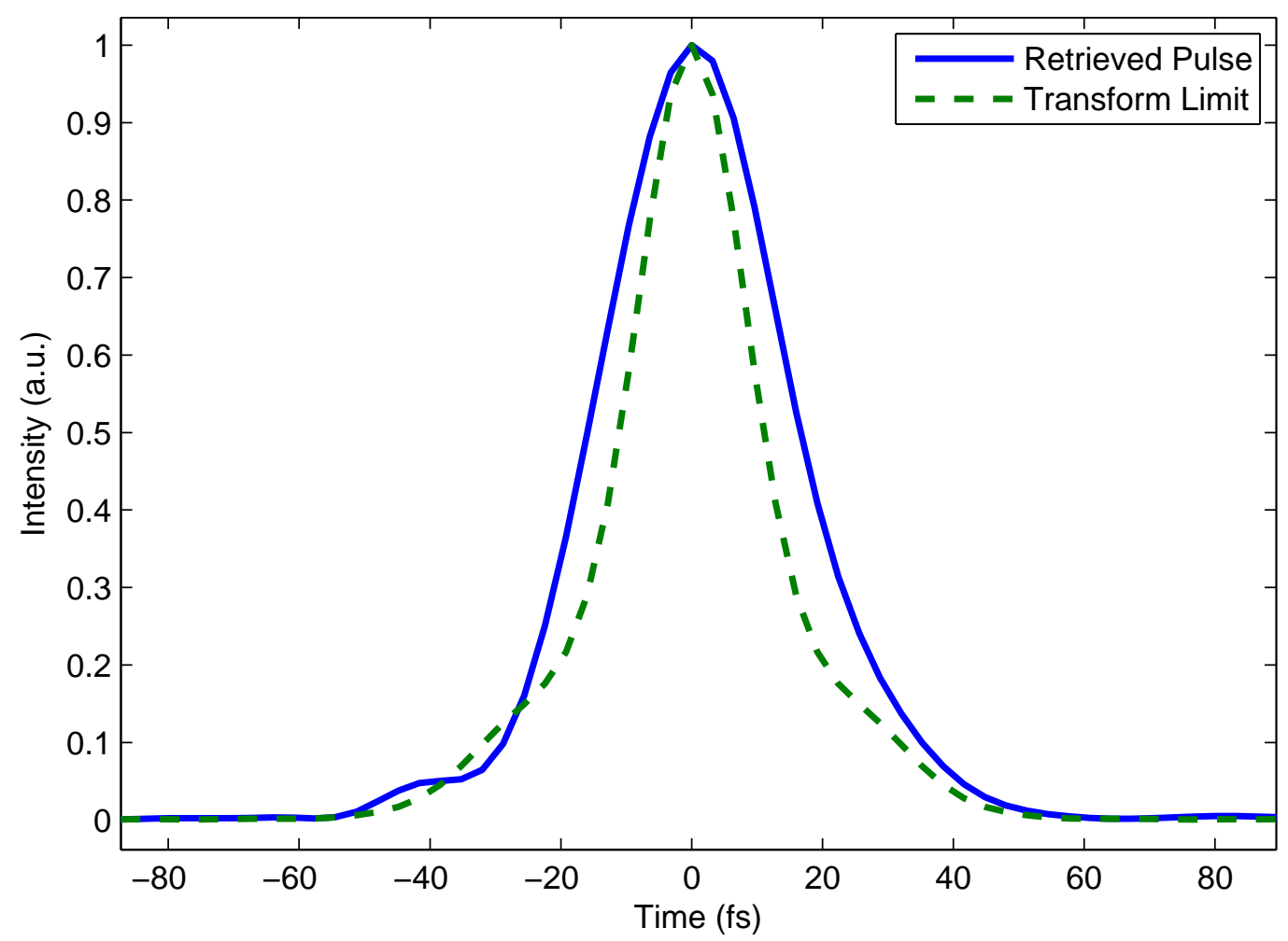

Figure C.4: A retrieved time domain profile of a nearly transform limited pulse (solid line) with a FWHM of $\sim 28$ fs. The dashed line shows the transform limit based on the pulse spectrum. 


\section{Bibliography}

[1] P. Baum and E. Riedle. Design and calibration of zero-additional-phase spider. Journal of the Optical Society of America B-optical Physics, 22(9):1875-1883, 2005.

[2] C. Iaconis and I. A. Walmsley. Self-referencing spectral interferometry for measuring ultrashort optical pulses. Ieee Journal of Quantum Electronics, 35(4):501-509, 1999.

[3] L. Lepetit, G. Cheriaux, and M. Joffre. Linear techniques of phase measurement by femtosecond spectral interferometry for applications in spectroscopy. Journal of the Optical Society of America B-optical Physics, 12(12):2467-2474, 1995. 


\section{APPENDIX D}

\section{PSII Sample Preparation}

The D1D2-cyt.b559 reaction centers studied in Chapter 3.8 were extracted from commercial spinach using a variation of the protocol of van Leeuwen et al. [1]. Spinach leaves were dessicated in a blender using a wash buffer and treated with Triton X-100 to dissociate the membrane proteins from other contents of the cell. Several centrifugation steps resulted in the isolation of BBY particles, a well-known preparation of the reaction center complex which is stable under storage at $-80^{\circ} \mathrm{C}[2]$.

The BBY particles were loaded onto an anion exchange column and incubated twice with Triton X-100 for 20 minutes to remove the antenna complexes CP47 and CP43. The detergent was thoroughly washed from the column and the sample was eluted with a high salt BTS-200 buffer. The eluted sample was confirmed to be the desired reaction center complex using the linear absorption spectrum. It is well-known [3] that D1D2-cyt.6559 reaction centers have a ratio of peak amplitudes in the Soret band of $A_{416} / A_{435}$ of 1.2. Presence of antenna complexes will change this ratio.

Because the eluted samples were rather dilute, it was necessary to reconcentrate them under centrifugation through a membrane filter (Millipore Amicon Ultra, pore size $10 \mathrm{kDa}$ ). This also allowed us to reduce the high salt concentration of the sample buffer. 


\begin{tabular}{|l|l|l|c|}
\hline Stock Solution & Mass & Volume $\mathrm{H}_{2} \mathrm{O}$ & $\mathrm{pH}$ (if not neutral) \\
\hline $0.5 \mathrm{M} \mathrm{HEPES}$ & $59,6 \mathrm{~g} \mathrm{HEPES}$ & $500 \mathrm{~mL}$ & 7.5 \\
\hline $4.0 \mathrm{M} \mathrm{NaCl}$ & $116.88 \mathrm{~g} \mathrm{NaCl}$ & $500 \mathrm{~mL}$ & \\
\hline $1.0 \mathrm{M} \mathrm{MgCl}$ & $10.2 \mathrm{~g} \mathrm{MgCl}{ }_{2} * 6 \mathrm{H}_{2} \mathrm{O}$ & $50 \mathrm{~mL}$ & \\
\hline $0.1 \mathrm{M} \mathrm{EDTA}$ & $1.9 \mathrm{~g} \mathrm{EDTA}$ & $50 \mathrm{~mL}$ & \\
\hline $0.5 \mathrm{M} \mathrm{MES}$ & $98.8 \mathrm{~g} \mathrm{MES}$ & $500 \mathrm{~mL}$ & 6.0 \\
\hline
\end{tabular}

Table D.1: Stock solutions used in the PSII RC prep

\section{PSII Sample Extraction Protocol}

In this section, the protocol for extracting D1D2-cyt.b559 reaction center complexes will be laid out in detail. To obtain enough sample for several experiments, it is recommended to begin with 2-3 packages of grocery spinach (10 oz. each). Several buffers and solvents will be required for different steps and the procedure. The recipes for these are all tabulated in Table D.2. The buffer recipes will only be discussed in the text when additional explanation is warranted. Stock solutions for making the buffers are detailed in Table D.1.

\section{Thylakoid Preparation}

The first part of the sample extraction separates the thylakoids from the rest of the spinach cell contents. The samples of interest are contained in the thylakoid membrane, along with the other components of the photosythesis engine.

\section{Homogenization}

Beginning with 2-3 bags (20-40 ounces) of fresh spinach leaves, wash the leaves in distilled water and then remove stems and veins by hand. A chilled blender should be prepared with $250 \mathrm{~mL}$ of homogenization buffer. If necessary, add more buffer to ensure that the blades are covered. Tear a handful of spinach leaves into smaller pieces and add them to the blender. Pulse the blender in 2-3 second bursts to allow all the solid material to fall into the blades. Add more leaves only after the current 
volume of material is well chopped. Once the blender is about half full of homogenized spinach, blend continuously for about 15 seconds.

Strain the homogenized spinach through four layers of cheesecloth, using a funnel to collect the fluid in a $2 \mathrm{~L}$ flask. Squeeze gently but thoroughly to collect as much fluid as possible without straining the solid remainder through the pores. Replace the cheesecloth as necessary in the case that gets too full or starts to tear. Discard the solid portion, and repeat this step several times until all the spinach is homogenized.

\section{First Centrifugation}

Pour the homogenate into $250 \mathrm{~mL}$ centrifuge bottles in pairs, being careful not to overfill since the bottles will sit at an angle in the rotor. It is very important to balance the weight between paired bottles to avoid damage to the centrifuge: place them on a scale to check the weight and use a pipette to make fine adjustments to the weight. In total, this step should use six centrifuge bottles for a total of about 1.5 L of sample. Place paired bottles across from each other in a Sorvall GSA rotor. Run the centrifuge for 10 minutes at $6500 \mathrm{rpm}$ at $4^{\circ} \mathrm{C}$.

\section{Resuspension in Wash Buffer}

Discard the supernatant and add a minimal amount of wash buffer (5-10 mL) to each bottle. Use the rubber policeman to resuspend the pellet in wash buffer. Pour the contents of all the bottles into a $55 \mathrm{~mL}$ homogenization tube. Rinse the bottles with wash buffer and pour this into the homogenization tube as well. Use a motorized homogenizer at about half the maximum speed to thoroughly mix the sample. The tube should be pulled back when the homogenizer is near, but not touching, the bottom, in order to apply some suction to larger particles that tend to settle at the bottom. Repeat this motion several times until the sample is thoroughly homogenized. Pour the contents of the tube into a beaker and fill with wash buffer 
to a total volume of $160 \mathrm{~mL}$. Split the solution evenly between four $40 \mathrm{~mL}$ centrifuge tubes.

\section{Second Centrifugation}

Place the centrifuge tubes in an SS-34 rotor and spin for 10 minutes at 10,000 rpm. This is the rotor used for the next several centrifugation steps. Discard the supernatant. At this point, the pellet consists of purified, washed thylakoid membranes, containing both photosystems.

\section{Photosystem II Preparation}

From this point onward, the samples will be exposed to detergent, which has the effect of stripping away many photoprotective elements and leaving the samples especially susceptible to photodamage. Therefore, it is important to work in the dark. Some of the detergents used are very powerful and will dissolve the samples completely if introduced in too high a concentration or for too much time, so care should be taken with buffer preparation and it is recommended to read several steps ahead in the prep.

\section{Suspension in Triton Buffer}

Resuspend the pellet in about $50 \mathrm{~mL}$ of Triton buffer. Pour the sample into a homogenization tube and homogenize very thoroughly. Rinse the tubes and homogenizer with Triton buffer and pour with sample into an Erlenmeyer flask. Measure and record the sample volume as $\mathrm{V}_{s}$.

\section{Calculation of Chlorophyll Content}

In order to determine how much detergent to add in the following step, it is first necessary to determine the concentration of chlorophyll in the sample. Pipette $10 \mu \mathrm{L}$ 
of sample solution into a small centrifuge tube. Add $5 \mathrm{~mL}$ of acetone to the tube to denature the protein, and mix with a vortex mixer. Using a tabletop centrifuge, spin at $1500 \mathrm{rpm}$ for 90 seconds.

Turn a spectrophotometer to transmittance mode and zero the instrument with a water blank $1 \mathrm{~cm}$ cuvette. Change to absorbance mode and zero at $663 \mathrm{~nm}$. Measure the absorbance of the denatured sample at $663 \mathrm{~nm}$ and $645 \mathrm{~nm}$, and record these values as $\mathrm{A}_{663}$ and $\mathrm{A}_{645}$, respectively. The chlorophyll content (in $\mathrm{mg}$ ) is calculated with the following equation:

The concentration of chlorophyll $(\mu \mathrm{g} / \mathrm{mL})$ in the acetone mixture is

$$
C_{c h l}=8.02 \cdot A_{663}+20.2 \cdot A_{645}
$$

The numerical coefficients contain the optical path length of the cuvette and the extinction coefficients at these wavelengths. The total mass of chlorophyll in the total sample volume is then determined to be

$$
M_{c h l}=\frac{C_{c h l}}{R_{d i l}} \times V_{s}
$$

where $\mathrm{V}_{s}$ is the entire sample volume measured in the previous step and $R_{d i l}=$ $V_{\text {chl }} / V_{\text {acetone }}$ is the dilution ratio, which should be $2 \mu \mathrm{L} / \mathrm{mL}$ by the above instructions but may vary if more or less sample is added. Note that the units are chosen to yield a final mass of chlorophyll in $\mathrm{mg}$ is $\mathrm{V}_{s}$ is in $\mathrm{mL}$.

The volume of Triton solution (25\% concentration) needed for the next step must be determined. The final concentration of Triton X-100 to chlorophyll should be $25 \mathrm{mg}$ Triton / $1 \mathrm{mg}$ Chl. Note here the distinction between the Triton detergent (referred to here as Triton X-100) and the detergent-infused buffer (Triton solution), which is a solution of Triton buffer containing a $0.25 \mathrm{~g} / \mathrm{mL}$ concentration of Triton $\mathrm{X}-100$. 
The mass ratio of Triton X-100 to chlorophyll should be 25:1. Therefore, the mass of Triton $\mathrm{X}-100$ required is

$$
M_{T r i}=25 \cdot M_{c h l}
$$

Because the detergent is diluted in the buffer at $25 \%(\mathrm{w} / \mathrm{v})$, the total volume of Triton solution needed is

$$
V_{\text {sol }}=\frac{M_{T r i}}{0.25}=4 M_{T r i}
$$

The net effect is that $1 \mathrm{~mL}$ of Triton solution should be used for every $10 \mathrm{mg}$ of chlorophyll.

\section{Triton Addition}

Pour the homogenized sample into a flask on ice and insert a stir bar. Begin stirring at a medium speed setting. With a $25 \mathrm{~mL}$ pipette, begin adding the Triton solution, slowly and carefully, one drop at a time. Dripping too quickly will result in local domains of high detergent concentration which can damage the sample. With a stopwatch, begin timing from the addition of the first drop of Triton. Incubate for 25 minutes.

During this time, clean the homogenizer and tube, and put these on ice with 12 small centrifuge tubes. Also place the SMN buffer in an ice bath.

\section{Third Centrfugation: Starch Removal}

Load the sample quickly into four small centrifuge tubes and spin at $3000 \mathrm{rpm}$ for 5 minutes. This step will collect the starch into a pellet.

\section{Fourth and Fifth Centrfugation: Triton Removal}

Keep the supernatant and collect into four more clean, chilled tubes. Centrifuge at 20,000 rpm for 30 minutes. Discard the supernatant, and resuspend the pellets in 
SMN buffer using the rubber policeman. Add SMN buffer to the same total volume as calculated in Equation D.4.

Separate the sample amongst four more centrifuge tubes and spin down for 30 minutes at 20,000 rpm. While the sample is spinning, prepare the BTS-400 buffer in the next step.

\section{BBY Particle Resuspension and Storage}

To prevent samples from aggregating, a small concentration of the detergent Ndodecyl maltoside (DM) should be added to a volume of BTS-400 buffer at a concentration of $0.05 \%(500 \mathrm{mg} / \mathrm{L})$. While the buffer should be prepared ahead of time, the DM should be added only just before use because it will cease working after a short time if mixed in too soon.

After the centrifugation is completed, discard the supernatant and resuspend the pellets in the BTS-400 buffer with DM. Determine the chlorophyll content following the same procedure used above. The samples should be stored at a chlorophyll concentration of $3 \mathrm{mg} / \mathrm{mL}$. Compute the total volume needed for the measured mass of chlorophyll and add BTS-400 buffer until this volume is reached. Mix gently and dispense the liquid into small glass bottles.

The current preparation at this stage consists of BBY particles, a stable subset of the PSII complex which can be easily stored at low temperatures for long times [2]. Label and store these in a $-80^{\circ} \mathrm{C}$ freezer.

\section{Tris Wash of BBY Particles}

To further purify the BBY particles before the final chromatography stages, it is helpful to wash the samples with a Tris buffer. Start with a small volume of sample $(\sim 10 \mathrm{~mL})$ as stored in the previous step. Thaw and add an equal volume of $1.6 \mathrm{M}$ Tris buffer. Incubate on ice under room lights for 20 minutes. 
Centrifuge the sample for 30 minutes at 20,000 rpm. Discard the supernatant and resuspend the pellet in BTS-200 buffer with $0.03 \%$ DM $(300 \mathrm{mg} / \mathrm{L})$. Once again, the DM should be added to the buffer only just before this step. The total volume at this step should be 1.5 times the starting volume so as to decrease the chlorophyll concentration from $3 \mathrm{mg} / \mathrm{mL}$ to $2 \mathrm{mg} / \mathrm{mL}$.

\section{D1D2-cyt.b559 Preparation: Column Chromatography}

The final process of the sample purification is to separate the D1D2-cyt.b559 core reaction center from the closely associated antenna complexes, including LHC II, CP43 and CP47, with the aid of an anion exchange column. The negatively charged D1D2-cyt.6559 particles. It should be noted that this stage of the preparation could be modified to yield other samples if desires, such as $\mathrm{CP} 47+\mathrm{RC}$ or free $\mathrm{CP} 47$, which

are interesting systems in their own right. That is, however, beyond the scope of this work and will not be discussed here in detail, though interested persons may wish to consult [4].

\section{Preparing the Column}

The chromatography column needs to be prepared only once, and if properly washed and stored, it can be used for multiple preparations. If an assembled column is already on hand, this section may be skipped. These instructions are for a $10 \mathrm{~mL}$ anion exchange column.

1. Measure out $10 \mathrm{~mL}$ of chemically modified carbohydrate beads (Q-Sepharose).

2. Dilute the medium with distilled water.

3. Place a filter in the bottom of the column and screw a loading tube to the bottom end. Use a post and clamp to secure the column in a vertical orientation.

4. Pour the diluted bead medium into the column, filling it to the top. 
5. Attach a loading tube with plunger to the top of the column. Screw the plunger down slightly.

6. Let the column settle for 15 minutes.

7. Attach a peristaltic pump or FPLC to the connector at the top of the bottom. Place a flask or beaker underneath to collect outgoing fluid.

8. Run BTS-200 buffer down the column at a rate of $4 \mathrm{~mL} /$ minute for 10 minutes.

9. Disconnect the pump, remove the top of the column, and drain off excess buffer carefully with a pipette.

10. Dampen a filter with distilled water and place in the top of the column, being careful to avoid trapping any air bubbles. Push the filter as flat as possible.

11. Screw the cap back on to the top of the column and attach it to the pump. Tighten down the plunger until it presses against the filter inside the column.

12. Run buffer down the column at a slowly increasing rate from $0.2-2.0 \mathrm{~mL} / \mathrm{min}$ and observe the output pressure to ensure the flow is not too high.

13. Disconnect and cap the ends to store the column, or prepare it for use in the next stage.

\section{Running the Column}

Start with a small volume of Tris-washed BBY particles (5-12 mL) in an ice bath. Measure out a volume of BTS-200 buffer equal to 1/7 (14.3\%) the volume of BBY sample. Add to this small volume of buffer a $10 \%$ concentration of DM (100 mg/mL). Turn off the lights and mix this solution with the sample. Swirl gently on ice for 10 minutes, then centrifuge the sample for 20 minutes at room temperature. Use an SE-12 rotor at 19,500 rpm. 
During the centrifugation step, connect the column to a peristaltic pump and submerge in an ice bath. Run the column output tube through a UV monitor and into a waste jar. Begin the FPLC system on the computer and click "Run" on any program present to go to monitoring view. Press "Manual" on the FPLC and change the valves to the "Wash position". Run the FPLC pumps at slow speed (0.01 $\mathrm{mL} / \mathrm{min})$. It is not necessary to actually use the FPLC to load the sample, as this can be performed more simply with the peristaltic pump. Activating the FPLC will, however, bring up an active display for the UV monitor, which is useful in detecting the presence of UV-absorbing substances like chlorophyll and Triton X-100 as they flow off the column.

Prepare $250 \mathrm{~mL}$ of BTS-200 buffer with 0.03\% (300 mg/L) DM. Set the peristaltic pump to a speed of $1.5 \mathrm{~mL} / \mathrm{min}$ and use this solution to wash the column. Run about 2 column volumes through at this speed. Keep the beaker of buffer in an ice bucket.

After centrifugation of the BBY particles, collect the supernatant into a roundbottomed vial. Once the washing of the column is finished, switch the pump inlet tube to the sample vial and load the sample onto the column at a speed of $1.5 \mathrm{~mL} / \mathrm{min}$. Once the sample is loaded, wash the column with the BTS-200+DM buffer. After about one column volume, the UV monitor should start registering large absorption from the fluid flowing off the column. This is due to unbound chlorophyll that is washing off. Continue to wash the column until the UV absorption drops to a low level.

Load the column with one full volume of BTS-200+Triton buffer. The functional fluid volume of this column is roughly $5 \mathrm{~mL}$. The volume of tubing from the sample to the top of the column is about $2.5 \mathrm{~mL}$. Therefore, $\sim 7.5 \mathrm{~mL}$ of buffer is required to completely cover the column. Alternatively, the UV monitor should register a high absorption reading from the Triton once it starts flowing off the column. Let the column sit for 20 minutes. Then, load a fresh volume of BTS-200+Triton buffer (5 
$\mathrm{mL})$. Incubate for another 20 minutes.

Wash the column with BTS-200+DM buffer until the UV absorption drops to nearly zero. This will indicate that all the Triton has been washed off the column. While washing, prepare $50 \mathrm{~mL}$ of High Salt BTS-200+DM buffer by adding 600 mg $\mathrm{MgSO}_{4}$ to fresh BTS-200+DM buffer, or else adding $0.03 \%$ DM to a previously prepared High Salt BTS-200 buffer.

After the wash step, it is time to elute the sample off the column. Run the High Salt BTS-200+DM buffer through the column and collect the eluted sample. A green band of reaction centers will travel down the column. The first several $\mathrm{mL}$ of collected sample will be colorless buffer and should be discarded. Once color is noticeable, however, the eluted sample should be collected in a series of numbered vials until no more color is visible. Different contaminants, including bound and unbound antenna complexes, will elute from the column at different rates, so it's important to note the order in which the vials are filled. To reduce the mixture of contaminants with the D1D2-cyt.b559 particles, it is recommended that each sample vial be filled with no more than about $1 \mathrm{~mL}$ of elution.

\section{Confirmation}

The eluted samples should be diluted to a $10 \%$ concentration with BTS-200+DM buffer and measured in a spectrophotometer. The purity of the sample can be measured by examining the ratio of absorbance from two peaks on the Soret region of the spectrum [3], located at $416 \mathrm{~nm}$ and 435, respectively. For pure reaction centers, the ratio should be $A_{416} / A_{435}=1.2$. Reaction centers with bound CP47 will exhibit a ratio of 0.9 , and unbound CP47 has a ration of 0.75 [3]. Several vials of eluted sample should be measured, since generally, some will contain more contaminants and others will hold purer sample. 


\begin{tabular}{|c|c|c|c|}
\hline Buffer/Solution & Ingredients & Volume & Stock Molarity \\
\hline Homogenization Buffer & $\begin{array}{l}50 \mathrm{mM} \text { HEPES } \\
0.4 \mathrm{M} \mathrm{NaCl} \\
2 \mathrm{mM} \mathrm{MgCl}_{2} \\
1 \mathrm{mM} \mathrm{EDTA}\end{array}$ & $\begin{array}{l}\frac{1 \mathrm{~L} \text { Total }}{100 \mathrm{~mL}} \\
100 \mathrm{~mL} \\
2 \mathrm{~mL} \\
10 \mathrm{~mL}\end{array}$ & $\begin{array}{l}0.5 \mathrm{M} \\
4.0 \mathrm{M} \\
1.0 \mathrm{M} \\
0.1 \mathrm{M}\end{array}$ \\
\hline Wash Buffer & $\begin{array}{l}50 \mathrm{mM} \text { HEPES } \\
0.15 \mathrm{M} \mathrm{NaCl} \\
5 \mathrm{mM} \mathrm{MgCl}_{2}\end{array}$ & $\begin{array}{l}\frac{1 \mathrm{~L} \text { Total }}{100 \mathrm{~mL}} \\
37.5 \mathrm{~mL} \\
5 \mathrm{~mL}\end{array}$ & $\begin{array}{l}0.5 \mathrm{M} \\
4.0 \mathrm{M} \\
1.0 \mathrm{M}\end{array}$ \\
\hline Triton Buffer & $\begin{array}{l}50 \mathrm{mM} \mathrm{MES} \\
15 \mathrm{mM} \mathrm{NaCl} \\
5 \mathrm{mM} \mathrm{MgCl}_{2} \\
\end{array}$ & $\begin{array}{l}\frac{400 \mathrm{~mL} \text { Total }}{40 \mathrm{~mL}} \\
1.5 \mathrm{~mL} \\
4 \mathrm{~mL}\end{array}$ & $\begin{array}{l}0.5 \mathrm{M} \\
4.0 \mathrm{M} \\
1.0 \mathrm{M}\end{array}$ \\
\hline Triton Solution & $\begin{array}{l}\text { Triton Buffer } \\
25 \%(\mathrm{w} / \mathrm{v}) \text { Triton X-100 }\end{array}$ & $\begin{array}{l}\frac{40 \mathrm{~mL} \text { Total }}{30 \mathrm{~mL}} \\
10 \mathrm{~g}\end{array}$ & \\
\hline SMN Buffer & $\begin{array}{l}50 \mathrm{mM} \text { MES } \\
10 \mathrm{mM} \mathrm{NaCl} \\
0.4 \mathrm{M} \text { Sucrose }\end{array}$ & $\begin{array}{l}\frac{1 \mathrm{~L} \text { Total }}{100 \mathrm{~mL}} \\
2.5 \mathrm{~mL} \\
136.92 \mathrm{~g}\end{array}$ & $\begin{array}{l}0.5 \mathrm{M} \\
4.0 \mathrm{M} \\
-\end{array}$ \\
\hline BTS-400 Buffer & $\begin{array}{l}20 \mathrm{mM} \mathrm{Bis}-T r i s \\
20 \mathrm{mM} \mathrm{MgCl}_{2} \\
5 \mathrm{mM} \mathrm{CaCl}_{2} \\
10 \mathrm{mM} \mathrm{MgSO}_{4} \\
0.4 \mathrm{M} \mathrm{Sucrose}\end{array}$ & $\begin{array}{l}100 \mathrm{~mL} \text { Total } \\
10 \mathrm{~mL} \\
2 \mathrm{~mL} \\
2.5 \mathrm{~mL} \\
10 \mathrm{~mL} \\
13.69 \mathrm{~g}\end{array}$ & $\begin{array}{l}0.2 \mathrm{M} \\
1.0 \mathrm{M} \\
0.2 \mathrm{M} \\
0.1 \mathrm{M} \\
-\end{array}$ \\
\hline BTS-200 Buffer & $\begin{array}{l}20 \mathrm{mM} \mathrm{Bis}^{-T r i s} \\
20 \mathrm{mM} \mathrm{MgCl}_{2} \\
5 \mathrm{mM} \mathrm{CaCl}_{2} \\
10 \mathrm{mM} \mathrm{MgSO}_{4} \\
0.2 \mathrm{M} \mathrm{Sucrose}^{2}\end{array}$ & $\begin{array}{l}500 \mathrm{~mL} \text { Total } \\
50 \mathrm{~mL} \\
10 \mathrm{~mL} \\
12.5 \mathrm{~mL} \\
50 \mathrm{~mL} \\
34.1 \mathrm{~g}\end{array}$ & $\begin{array}{l}0.2 \mathrm{M} \\
1.0 \mathrm{M} \\
0.2 \mathrm{M} \\
0.1 \mathrm{M} \\
-\end{array}$ \\
\hline BTS-200 + Triton & $\begin{array}{l}20 \mathrm{mM} \mathrm{Bis}^{-T r i s} \\
20 \mathrm{mM} \mathrm{MgCl}_{2} \\
5 \mathrm{mM} \mathrm{CaCl}_{2} \\
10 \mathrm{mM} \mathrm{MgSO}_{4} \\
0.2 \mathrm{M} \mathrm{Sucrose} \\
10 \%(\mathrm{w} / \mathrm{v}) \text { Triton X-100 }\end{array}$ & $\begin{array}{l}100 \mathrm{~mL} \text { Total } \\
10 \mathrm{~mL} \\
2 \mathrm{~mL} \\
2.5 \mathrm{~mL} \\
10 \mathrm{~mL} \\
6.83 \mathrm{~g} \\
10 \mathrm{~g}\end{array}$ & $\begin{array}{l}0.2 \mathrm{M} \\
1.0 \mathrm{M} \\
0.2 \mathrm{M} \\
0.1 \mathrm{M} \\
-\end{array}$ \\
\hline High Salt BTS-200 & $\begin{array}{l}\text { BTS-200 Buffer } \\
0.1 \mathrm{M} \mathrm{MgSO}_{4}\end{array}$ & $\begin{array}{l}\frac{50 \mathrm{~mL} \text { Total }}{50 \mathrm{~mL}} \\
602 \mathrm{mg}\end{array}$ & \\
\hline
\end{tabular}

Table D.2: A list of recipes for all buffers used in the D1D2-cyt.b559 sample preparation. 


\section{Bibliography}

[1] P.J. van Leeuwen, M.C. Nieveen, E.J. van de Meent, J.P. Dekker, and H.J. van Gorkom. Rapid and simple isolation of pure photosystem ii core and reaction center particles from spinach. Photosynthesis Research, 28(3):149-153, 1991.

[2] DA Berthold, GT Babcock, and CF Yocum. A highly resolved, oxygenevolving photosystem-ii preparation from spinach thylakoid membranes - electronparamagnetic-res and electron-transport properties. Febs Letters, 134(2):231-234, 1981.

[3] C Eijckelhoff, H vanRoon, ML Groot, $\mathrm{R}$ vanGrondelle, and JP Dekker. Purification and spectroscopic characterization of photosystem ii reaction center complexes isolated with or without triton x-100. Biochemistry, 35(39):12864-12872, OCT 11996.

[4] J. P. Dekker, N. R. Bowlby, and C. F. Yocum. Chlorophyll and cytochrome-b559 content of the photochemical-reaction center of photosystem-ii. Febs Letters, 254(1-2):150-154, 1989. 


\section{APPENDIX E}

\section{Sample Cell}

In order to perform experiments at low temperatures, it was necessary to design a custom sample cell shaped to fit in our cryostat chamber (Oxford Instruments Microstat N). There were several requirements to fulfil in designing the cell: it must be of small enough diameter to fit in the narrow space of the cryostat while maximizing the viewable window area; the thickness must be low and preferably adjustable; the total volume should be low to minimize the use of precious samples; and the cell must maintain a seal under vacuum and at low temperatures.

Early sample designs incorporated cell windows that were fixed in place with epoxy. These fulfilled the aforementioned requirements well, but it was found that certain dye solutions were optically degrading, such that the optical density was far reduced after only a few hours or days. For robust laser dyes, especially, this result is shocking, as was the appearance of additional peaks in the linear spectrum, presumably from chemical products of some reaction in the dye or solvent. After thorough testing, it was determined that the epoxy was interacting with the sample and degrading the dye molecules. Even though the area of contact with the epoxy was small, it was enough to cause a major effect. Therefore, a new requirement was added: that the sample should only come in contact with materials that were more 
or less chemically inert.

To this end, I designed a sample cell, based on the concepts presented in [1], which uses only compression to maintain a seal, so no adhesives are necessary. The lid and lower base are made of aluminum. To aid in seal formation, a small amount of vacuum grease is applied to two $10 \mathrm{~mm}$ diameter, $1 \mathrm{~mm}$ thick viton o-rings, which are then inserted into the lid and base. These o-rings form the outer compression seal between the metal pieces and the glass windows, while a larger, ungreased \# 013 o-ring forms the seal between the two metal pieces.

To load the sample, the o-rings are put into place before inserting a $0.25 \mathrm{~mm}$ thick, $10 \mathrm{~mm}$ diameter glass window into the central cavity. A teflon ring spacer is placed on top of the window. The thickness of this spacer can be varied to adjust the final optical path length of the sample. Using a pipette, roughly $35 \mu \mathrm{L}$ of sample is then deposited onto the center of the window. A second window (10 $\mathrm{mm}$ diameter, $1 \mathrm{~mm}$ thickness) is lowered carefully on top of the sample to avoid trapping bubbles. The design of the cell provides overflow space so that pressure is not applied to the windows if too much sample volume is loaded. the lid is then applied and tightened on with six $0-80$ screws from the top. Care must be taken to tighten the screws in a non-radial pattern so as to keep the pressure relatively even across the window, as over-tightening can crack the window or compromise the seal.

This cell has been used successfully at pressures as low as $10^{-4}$ Torr and temperatures down to $77 \mathrm{~K}$. All components are reusable, though the o-rings should be replaced periodically. The design is shown in Figure E.1. 
(a)

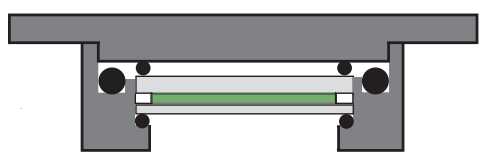

(c)

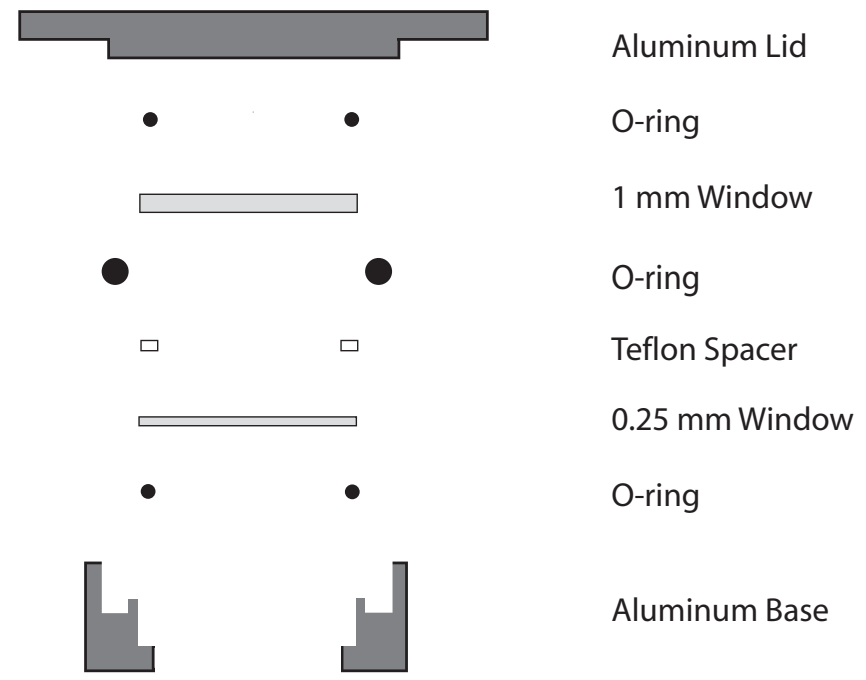

(b)

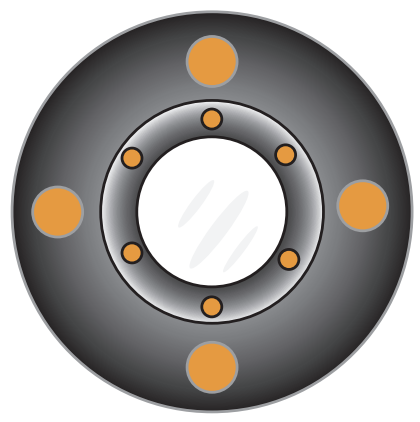

Figure E.1: Design of a low-temperature, vacuum-rated sample cell using only compression fittings. A cross-section is shown in (a) with the sample space colored green. (b) is a top view of the closed cell, and (c) gives a blownapart view of the components. 


\section{Bibliography}

[1] K. Matsuo, K. Sakai, Y. Matsushima, T. Fukuyama, and K. Gekko. Optical cell with a temperature-control unit for a vacuum-ultraviolet circular dichroism spectrophotometer. Analytical Sciences, 19(1):129-132, 2003. 